\title{
Terminierungskinetik radikalischer Homo- und Copolymerisationen bis zu hohen Monomerumsätzen
}

\author{
Dissertation \\ zur Erlangung des Doktorgrades \\ der Mathematisch-Naturwissenschaftlichen Fakultäten \\ der Georg-August-Universität zu Göttingen
}

vorgelegt von

Achim Feldermann

aus Bremen

Göttingen 2003 
D 7

Referent:

Prof. Dr. M. Buback

Korreferent:

Prof. Dr. K. Hoyermann

Tag der mündlichen Prüfung:

03.07.2003 


\section{INHALTSVERZEICHNIS}

1 ZUSAMMENFASSUNG 1

2 Einleitung

3 TheORETISCHE GRUNDLAGEN

3.1 Ideale Polymerisationskinetik 7

3.2 Umsatzabhängigkeit der Terminierungsreaktion 9

$\begin{array}{ll}3.3 \text { Kettenübertragungsreaktionen } & 13\end{array}$

3.4 Literatur Kapitel $3 \quad 14$

4 EXPERIMENTELle TECHNIKEN

4.1 Hochdruckmesszelle $\quad 15$

$\begin{array}{ll}4.2 \text { Druckerzeugung } & 17\end{array}$

4.3 Temperaturregelung der optischen Hochdruckzellen 18

$\begin{array}{ll}4.4 \mathrm{IR} / \mathrm{NIR}-\text { Spektrometer } & 18\end{array}$

$\begin{array}{ll}4.5 \text { Detektoren } & 19\end{array}$

4.6 Messanordnung für Experimente mit zeitaufgelöster Umsatzdetektion (SP-PLP) 19

4.7 Auswertung der Signale $\quad 22$

4.8 Gelpermeationschromatographie 25

4.9 Literatur Kapitel $4 \quad 25$

5 DURCHFÜHRUNG DER EXPERIMENTE 26

5.1 Vorbereitung der Substanzen 26

5.2 Auswahl der Messbedingungen $\quad 29$

5.2.1 Messbedingungen der zeitaufgelösten Messung 29

$\begin{array}{ll}5.2 .2 \text { Vorpolymerisation } & 30\end{array}$

5.3 Ablauf eines typischen zeitaufgelösten Experiments 32

5.4 Ablauf der Vorpolymerisation 33

5.5 Fehlerbetrachtung 33

5.6 Literatur Kapitel 5 
6 MMA-HOMOPOLYMERISATIONEN

6.1 Vorbemerkungen zur Auswertung 35

6.1.1 Signal-Rausch-Verhältnis 35

6.1.2 Homogenität der Reaktionsmischung 37

6.2 Erweiterung des zugänglichen Umsatzbereiches 38

6.3 Untersuchungen im Bereich der „Reaktionsdiffusionskontrolle“ 42

6.4 Untersuchungen mit dem Transferagens DDM 45

6.5 Untersuchungen zur Kettenlängenabhängigkeit von $k_{\mathrm{t}}$ mit DMPA 49

6.6 Literatur Kapitel 6

7 COPOLYMERISATIONEN

7.1 Bestimmung von $k_{\mathrm{t}}$ aus SP-PLP-Experimenten $\quad 54$

$\begin{array}{ll}7.2 \text { Ergebnisse und Modellierungen } & 61\end{array}$

$\begin{array}{ll}7.3 \text { Literatur Kapitel } 7 & 65\end{array}$

8 KETTENLÄNGENABHÄNGIGKEIT VON $\boldsymbol{k}_{\mathrm{t}} \quad 66$

8.1 Auswahl des Photoinitiators 66

8.2 Auswertung der Messsignale $\quad 68$

8.3 Ergebnisse und Diskussion 83

8.3.1 Terminierungsgeschwindigkeitskoeffizient $k_{\mathrm{t}}{ }^{0} \quad 83$

8.3.2 Parameter $\alpha$ bei niedrigen Monomerumsätzen 86

8.3.3 $\alpha$ für Methacrylate bis zu höheren Monomerumsätzen 88

8.3.4 $\alpha$ für Acrylate bis zu höheren Monomerumsätzen 89

8.3.5 DA und BA in überkritischem $\mathrm{CO}_{2}$

$\begin{array}{ll}\text { 8.3.6 } \alpha \text { für MA-DA Copolymerisationen } & 106\end{array}$

$\begin{array}{ll}\text { 8.5 Schlussfolgerungen } & 107\end{array}$

$\begin{array}{ll}\text { 8.6 Literatur Kapitel } 8 & 111\end{array}$

9 Ausblick 114

10 Anhang 116

10.1 Verzeichnis der Abkürzungen 116

10.2 Betriebsanweisung der Anlage zur Aufnahme zeitaufgelöster IR- Signale $\quad 119$ 


\section{ZUSAMMENFASSUNG}

Die Terminierungskinetik radikalischer Homo- und Copolymerisationen wurde mit der SP-PLP-Technik bis zu hohen Monomerumsätzen untersucht. Durch die Modifizierung der bestehenden SP-PLP-Anlage konnte die pro Messsignal zur Verfügung stehende Datenpunktanzahl sowie die Genauigkeit deutlich gesteigert werden.

Für Methylmethacrylat (MMA) konnte durch Hinzufügen einer Vorpolymerisation der Photoinitiatorverbrauch und das Problem von Inhomogenität in der Reaktionsmischung verringert werden. Auf diese Weise ließ sich der Bereich, in dem SP-PLP-Messungen durchgeführt werden können, auf bis etwa $60 \%$ Monomerumsatz erweitern. Als die günstigste Methode erwies sich eine rein thermische Vorpolymerisation ohne zusätzlichen Initiator. In Übereinstimmung mit durch andere Techniken erhaltenen Literaturdaten wurde die Reaktionsdiffusionskonstante mit $C_{\mathrm{RD}}=95 \pm 40$ bestimmt. Mit der Zugabe des Übertragungsagens Dodecylmercaptan (DDM) zur Reaktionsmischung wurde die Bedeutung der Polymermatrix auf die Kinetik durch ein Ausbleiben des Geleffekts mit konstant hohem Terminierungsgeschwindigkeitskoeffizienten $k_{\mathrm{t}}$ gezeigt.

Es wurden die Copolymerisationen der Systeme Methylmethacrylat-Dodecylacrylat und Dodecylmethacrylat-Methylacrylat diskutiert. Dabei ergab sich durch die stark unterschiedlichen Copolymerisationsparameter $r_{\mathrm{i}}$ der beiden Monomere eine Abhängigkeit des Wachstumsgeschwindigkeitskoeffizienten $k_{\mathrm{p}}$ vom Umsatz, was bei der Bestimmung des Terminierungsgeschwindigkeitskoeffizienten $k_{\mathrm{t}}$ aus den bei SP-PLP-Messungen erhaltenen gekoppelten Parameter $k_{\mathrm{t}} / k_{\mathrm{p}}$ zu beachten ist. Die so erhaltenen Werte für die Copolymerisationen, $k_{\mathrm{t}, \mathrm{copo}}$, lassen sich mit einem ,penultimate“ Modell beschreiben, bei dem zur Reduzierung der Zahl der Koeffizienten eine geometrischen Mittelung einiger Terminierungsgeschwindigkeitskoeffizienten vorgenommen wird.

Durch den Einsatz des Photoinitiators $\alpha$-Methyl-4-(methyl-mercapto)- $\alpha$-morpholinopropiophenon (MMMP) konnte die Kettenlängenabhängigkeit von $k_{\mathrm{t}}$ für verschiedene Monomere aus einzelnen Messsignalen bis zu höheren Monomerumsätzen untersucht werden. Zur Auswertung wurde die Kettenlängenabhängigkeit über das Potenzgesetz $k_{\mathrm{t}}^{\mathrm{i}, \mathrm{i}}=k_{\mathrm{t}}^{0} \cdot i^{-\alpha}$ modellabhängig bestimmt, wobei $i$ die Kettenlänge, $k_{\mathrm{t}}^{0}$ der Terminierungsgeschwindigkeitskoeffizient für sehr kleine Radikale und $\alpha$ das Ausmaß der Kettenlängenabhängigkeit ist. Für die untersuchten Methacrylate ergab sich bei niedrigen Umsätzen ein Parameter $\alpha$ um 0.15. $\mathrm{Zu}$ höheren Umsätzen hin steigt $\alpha$ deutlich an, was auf die Bestimmung von $k_{\mathrm{t}}$ durch andere 
Diffusionsprozesse als zu Beginn der Polymerisation hindeutet. Acrylate zeigen aufgrund der höheren Reaktivität und größeren Flexibilität der Makroradikale ein abweichendes Verhalten. Bei diesen Monomeren nimmt $\alpha$ mit der Anzahl von abstrahierbaren Wasserstoffatomen in der Esterseitenkette bzw. der Stabilität der durch Abstraktion gebildeten Radikale und dem Monomerumsatz zu. Für niedrige Umsätze ergibt sich für Acrylate mit wenig verfügbaren Wasserstoffatomen analog den Methacrylaten ein Wert um $\alpha=0.15$. Bei höheren Umsätzen oder bei vielen abstrahierbaren H-Atomen erreicht $\alpha$ Werte bis 0.43 . Der hohe Wert von $\alpha$ deutet auf einen großen Anteil von wachsenden Spezies mit der Radikalfunktion in der Mitte der Kette (,mid-chain“ Radikale) hin. Diese Radikale werden durch Übertragungsreaktionen gebildet, deren Wahrscheinlichkeit mit der Anzahl von verfügbaren H-Atomen und dem Umsatz zunimmt. In Einklang mit dieser Erklärung stehen Resultate aus SP-PLPExperimenten mit BA bei Zusatz von überkritischem $\mathrm{CO}_{2}$ als Lösungsmittel. Es zeigt sich für dieses Monomer, bei dem die Kettenlängenabhängigkeit von $k_{\mathrm{t}}$ in Substanz gering ist, eine deutliche Steigerung des Anteils von „mid-chain“ Radikalen und damit auch von $\alpha$. Bei Dodecylacrylat mit bereits erreichtem Grenzwert von $\alpha$ hingegen führt die Lösungsmittelzugabe zu keiner Veränderung dieses Parameters. 


\section{EINLEITUNG}

Polymere Werkstoffe sind in der modernen Welt allgegenwärtig. Die Produktionszahlen dieser Substanzklasse liegen im Bereich von Millionen Jahrestonnen. Dabei sind Polyacrylate und Copolymere von Acrylaten und Methacrylaten besonders für die Herstellung von Klebstoffen, Lacken und Coatings von großer Bedeutung. Aufgrund der engen Verknüpfung von Produktstruktur und Reaktionskinetik bei Polymerisationen ist zur Optimierung der Polymereigenschaften sowie der Herstellungsverfahren die Kenntnis von kinetischen Koeffizienten von entscheidender Wichtigkeit.

Die Bestimmung des Geschwindigkeitskoeffizienten für die Terminierungsreaktion $k_{\mathrm{t}}$ ist eine besondere Herausforderung, da dieser Koeffizient neben Druck und Temperatur von weiteren Parametern wie Monomerumsatz, Viskosität der Reaktionsmischung und Kettenlänge der Radikale abhängt. Die SP-PLP-Technik (Single Pulse-Pulsed Laser Polymerization) stellt die zur Zeit weitreichendste Methode zur experimentellen Bestimmung von $k_{\mathrm{t}}$ dar. Dabei wird durch Anregung eines Photoinitiators mittels eines kurzen Laserpulses eine Radikalpopulation erzeugt und anschließend die Abnahme der Monomerkonzentration NIR-spektroskopisch mit einer Zeitauflösung im Mikrosekundenbereich verfolgt. Aus der Analyse der Signale ergibt sich das Verhältnis von Terminierungs- zu Wachstumsgeschwindigkeitskoeffizient, $k_{\mathrm{t}} / k_{\mathrm{p}}$, als Funktion des Monomerumsatzes. Zusammen mit unabhängig bestimmten Daten für $k_{\mathrm{p}}$ ist somit $k_{\mathrm{t}}$ zugänglich, wobei $k_{\mathrm{t}}$ ein kettenlängengemittelter Wert ist. Ursprünglich für die Untersuchung der Polymerisation von Ethen entwickelt [1], wurde diese Technik in den letzten Jahren für zahlreiche Studien verschiedener Homo- und Copolymerisationen von Acrylaten und Methacrylaten angewendet [2, 3, 4, 5, 6, 7, 8, 9].

Die Qualität der Messsignale verbessert sich mit steigendem $k_{\mathrm{p}}$ und fallendem $k_{\mathrm{t}}$. Innerhalb der Familie der Acrylate und Methacrylate ist Methylmethacrylat (MMA) somit wegen des kleinen Wachstumsgeschwindigkeitskoeffizienten $k_{\mathrm{p}}$ bei gleichzeitig sehr großem $k_{\mathrm{t}}$ für die SP-PLP-Technik das am wenigsten geeignete Monomer. Durch den geringen Umsatz pro Laserpuls ergibt sich ein ungünstiges Signal-Rausch-Verhältnis. Andererseits besteht aus industrieller Sicht ein großes Interesse an kinetischen Daten für MMA. Polymethylmethacrylat zeichnet sich durch günstige optische Eigenschaften sowie hohe mechanische Belastbarkeit aus und wird beispielsweise zur Herstellung von Plexiglas ${ }^{\circledR}$ verwendet. Auch aus akademischer Sicht ist MMA ein wichtiges Monomer. Es ist bekannt, dass der Terminierungsgeschwindigkeitskoeffizient für dieses Methacrylat mit steigendem Umsatz um 
einige Größenordnungen abnimmt [10]. Somit bietet sich MMA für mechanistische Studien zur Terminierungskinetik in weiten Umsatzbereichen an. Bislang war mit der SP-PLPTechnik wegen des geringen Umsatzes pro Laserpuls und dem damit verbundenen hohen Verbrauch an Photoinitiator die Bestimmung von $k_{\mathrm{t}}$ für MMA nur bis etwa $20 \%$ Monomerumsatz möglich. Ein Ziel dieser Arbeit war es deshalb, den zugänglichen Umsatzbereich zu erweitern und die Terminierungskinetik von MMA bis zu hohen Umsätzen $\mathrm{zu}$ untersuchen. Um das Problem des Initiatorverbrauchs $\mathrm{zu}$ umgehen, soll vor dem eigentlichen SP-PLP-Experiment eine Polymerisation ohne Reduzierung der Photoinitiatorkonzentration durchgeführt werden.

Für ein tieferes Verständnis der Terminierungskinetik sind Studien zur Copolymerisation ein wichtiger Aspekt. Für die Terminierungsreaktion wird im Wesentlichen von einer Geschwindigkeitskontrolle durch Diffusionsprozesse ausgegangen. Im Fall von Copolymerisationen kann zusätzlich der Einfluss der letzten und vorletzten Kettenglieder auf $k_{\mathrm{t}}$ studiert und die Gültigkeit entsprechender Modelle überprüft werden. Deshalb wurden bereits einige Acrylat-Acrylat sowie Methacrylat-Methacrylat Systeme hinsichtlich $k_{\mathrm{t}}$ untersucht $[5,6,11]$. Auch für Copolymerisationen von Acrylaten mit Methacrylaten wurden in der Vergangenheit Daten aus SP-PLP-Experimenten gewonnen [5, 8]. Für solche Systeme ist jedoch bekannt, dass die wachsende Kette bevorzugt mit dem Methacrylat reagiert und demzufolge im Copolymer ein höherer Anteil an Methacrylat im Vergleich zur Monomermischung resultiert [12,6]. Als Folge dieses Verhaltens verarmt die Monomermischung somit an Methacrylat. In dieser Arbeit soll untersucht werden, wie sich diese Erniedrigung des Methacrylat-Anteils auf die Kinetik der Copolymerisation auswirkt. Dazu ist es notwendig, jederzeit während der Polymerisation die aktuelle Monomerzusammensetzung $\mathrm{zu}$ berechnen und die Geschwindigkeitskoeffizienten für Wachstums- und Terminierungsreaktion zu bestimmen.

Bei den hier vorgestellten Untersuchungen zur MMA-Homopolymerisation und zur Copolymerisation von Methacrylat-Acrylat Systemen wird von der idealen Polymerisationskinetik ausgegangen. Dabei wird angenommen, dass $k_{\mathrm{t}}$ von der Kettenlänge der terminierenden Radikale unabhängig ist. In der Literatur wird jedoch seit einigen Jahren eine Kettenlängenabhängigkeit von $k_{\mathrm{t}}$ diskutiert $[13,14,15,16]$ und in Polymerisationen einiger Monomere bei meist niedrigen Umsätzen auch beobachtet [17, 18, 19, 20, 21]. Bei diesen Untersuchungen zeigte sich, dass $k_{\mathrm{t}}$ mit zunehmender Kettenlänge abnimmt. Es wurden auch mit der SP-PLP-Technik bereits Experimente zur Bestimmung dieser Kettenlängen- 
abhängigkeit durchgeführt [20]. Dabei wurde die Nichtidealität des verwendeten Photoinitiators DMPA (2,2-Dimethoxy-2-phenylacetophenon) ausgenutzt. Dieser Initiator liefert zwei Radikalfragmente unterschiedlicher Aktivität. Während eines der entstehenden Radikale eine große Reaktivität bezüglich der Addition an das Monomer zeigt, nimmt das andere Radikal nur an Terminierungsreaktionen teil und wirkt somit wie ein Inhibitor. Durch Experimente mit unterschiedlichen DMPA-Anfangskonzentrationen bei ansonsten gleichen Bedingungen lassen sich die SP-PLP-Signale hinsichtlich einer Kettenlängenabhängigkeit von $k_{\mathrm{t}}$ auswerten. Dieses Verfahren ist jedoch kompliziert und die Durchführung der entsprechenden Messungen unter bis auf die Initiatorkonzentration gleichen Bedingungen gerade bei höheren Monomerumsätzen schwierig. Deshalb ist der Einsatz eines Photoinitiators mit gleicher Aktivität beider Radikalfragmente und Analyse der Kettenlängenabhängigkeit von $k_{\mathrm{t}}$ aus einzelnen Signalen von Vorteil. Der von Külpmann [22] für die SP-PLP-Technik „entdeckte“ Photoinitiator MMMP ( $\alpha$-Methyl-4-(methyl-mercapto)- $\alpha$-morpholinopropiophenon) in Verbindung mit einer entsprechenden Auswerteprozedur erlaubt die Bestimmung der Kettenlängenabhängigkeit von $k_{\mathrm{t}}$ aus einzelnen SP-PLP-Signalen. In dieser Arbeit soll diese Methodik für verschiedene Monomere angewendet und die Kettenlängenabhängigkeit von $k_{\mathrm{t}}$ bis $\mathrm{zu}$ hohem Umsatz studiert werden. Weitere Erkenntnisse können dabei Untersuchungen in Lösung sowie von Copolymerisationen liefern. Es muss zunächst diskutiert werden, inwieweit die Verwendung von Modellen zur Terminierungsreaktion notwendig ist und ob es die Güte der Messsignale erlaubt, eine Modelldiskriminierung durchzuführen.

\section{Literatur Kapitel 2}

[1] M. Buback, H. Hippler, J. Schweer, H.-P. Vögele, Makromol. Chem., Rapid Commun. 1986, 7, 261.

[2] C. Kurz, Dissertation, Göttingen 1995.

[3] C. Schmaltz, Dissertation, Göttingen 1997.

[4] M. Buback, C. Kowollik, Macromolecules 1998, 31, 3221.

[5] M. Buback, C. Kowollik, Macromolecules 1999, 32, 1445.

[6] C. Kowollik, Dissertation, Göttingen 1999.

[7] M. Buback, C. Kowollik, C. H. Kurz, A. Wahl, Macromol. Chem. Phys. 2000, 201, 464.

[8] M. Buback, A. Feldermann, Austr. J. Chem. 2002, 55, 475. 
[9] D. Nelke, Dissertation, Göttingen 2002.

[10] R. Sack-Kouloumbris, G. Meyerhoff, Macromol. Chem. 1989, 190, 1133.

[11] K. F. O’Driscoll, K. Ito, J. Polym. Sci. 1979, 17, 3913.

[12] R. A. Hutchinson, J. H. McMinn, D. A. Paquet Jr., S. Beuermann, C. Jackson, Ind. Eng. Chem. Res. 1997, 36, 1103.

[13] A. R. Khokhlov, Macromol. Chem., Rapid Commun. 1981, 2, 633.

[14] P. G. de Gennes, J. Chem. Phys. 1982, 76, 3316 und 3322.

[15] B. Friedman, B. O’Shaughnessy, Macromolecules 1993, 26, 5726.

[16] M. Buback, C. Barner-Kowollik, M. Egorov, V. Kaminsky, Macromol. Theory Simul. 2001, 10, 209.

[17] H. K. Mahabadi, Macromolecules 1985, 18, 1319.

[18] O. F. Olaj, G. Zifferer, G. Gleixner, Macromol. Chem., Rapid Commun. 1985, 6, 773.

[19] J. B. L. de Kock, B. Klumpermann, A. M. van Herk, A. L. German, Macromolecules 1997, 30, 6743.

[20] M. Buback, M. Busch, C. Kowollik, Macromol. Theory Simul. 2000, 9, 442.

[21] P. Vana, T. P. Davis, C. Barner-Kowollik, Macromol. Rapid Commun. 2002, 23, 952.

[22] A. Külpmann, Diplomarbeit, Göttingen 2000. 


\section{THEORETISCHE GRUNDLAGEN}

\subsection{Ideale Polymerisationskinetik}

Die Beschreibung einer idealen Polymerisation beruht auf vier Annahmen:

- Alle Reaktionen verlaufen irreversibel

- Monomeres wird nur im Wachstumsschritt verbraucht

- Alle Radikale haben die gleiche Reaktivität, unabhängig von ihrer Kettenlänge

- Terminierung erfolgt nur durch Disproportionierung oder Radikalkombination, nicht durch Transferreaktionen

Das durch die obigen Annahmen vereinfachte Reaktionsschema sieht folgendermaßen aus:

$\begin{array}{llc}\text { Initiierung: } & \mathrm{I} \rightarrow 2 \mathrm{R} \cdot & k_{\mathrm{d}} \\ \text { Wachstum: } & \mathrm{R} \cdot+\mathrm{M} \rightarrow \mathrm{R} . & k_{\mathrm{p}} \\ \text { Terminierung: } & \mathrm{R} \cdot+\mathrm{R} \cdot \rightarrow \mathrm{P} & k_{\mathrm{t}}\end{array}$

Die Initiierung ist auf thermischem, chemischem oder photochemischem Wege möglich. Dabei kann direkt das Monomer oder ein Initiator zum Zerfall angeregt werden. Im Rahmen der vorliegenden Arbeit wird, außer bei der thermisch initiierten Vorpolymerisation, ausschließlich der Fall behandelt, bei dem die Initiierung photochemisch durch Anregung eines Initiators mit UV-Laserpulsen erfolgt. Bei einer Halbwertsbreite der Pulse von ca. 20 ns kann die Bildung der Radikale im Vergleich zu den nachfolgenden Wachstums- und Terminierungsschritten als augenblicklich angesehen werden. Die durch einen Laserpuls erzeugte Radikalkonzentration $c_{\mathrm{R}}{ }^{0}$ ergibt sich als:

$$
c_{\mathrm{R}}^{0}=2 \cdot \Phi \cdot \frac{n_{\mathrm{abs}}}{V}
$$

mit der Primärquantenausbeute $\Phi$, der Anzahl der absorbierten Photonen $n_{\text {abs }}$ und dem bestrahlten Volumen $V$. Die Primärquantenausbeute ist das Produkt aus der Lasereffektivität $\varphi$ (Anteil der absorbierten Photonen, die zur Bildung von Radikalen führen) und der Initiatoreffektivität $f$, mit der beschrieben wird, welcher Anteil der gebildeten Initiatorradikale tatsächlich eine Polymerkette startet. Bei Gültigkeit des Lambert-Beerschen Gesetzes lässt sich die Zahl der absorbierten Photonen berechnen: 


$$
n_{\mathrm{abs}}=\frac{E_{\mathrm{P}}}{E_{\lambda}} \cdot\left(1-10^{-\varepsilon \cdot c \cdot d}\right)
$$

$E_{\mathrm{P}}: \quad$ Energie eines Laserpulses

$E_{\lambda}$ : Energie eines Mols Photonen bei der Laserwellenlänge $\lambda$

$\varepsilon: \quad$ molarer dekadischer Absorptionskoeffizient des Initiators bei dieser Wellenlänge

$c$ : Konzentration des Initiators

$d: \quad$ durchstrahlte Schichtlänge

Im Wachstumsschritt lagert sich ein Monomermolekül an ein Radikal an. Die Änderung der Monomerkonzentration wird durch folgendes Geschwindigkeitsgesetz beschrieben:

$$
\frac{\mathrm{d} c_{\mathrm{M}}}{\mathrm{d} t}=-k_{\mathrm{p}} \cdot c_{\mathrm{M}} \cdot c_{\mathrm{R}}
$$

$k_{\mathrm{p}}$ : Wachstumsgeschwindigkeitskoeffizient

$c_{\mathrm{R}}$ : Radikalkonzentration

$c_{\mathrm{M}}: \quad$ Monomerkonzentration

Bei Copolymerisationen ist diese Monomerkonzentration die Summe der Konzentrationen der Monomere. Die Terminierung erfolgt durch Rekombination oder Disproportionierung. Beide Möglichkeiten werden in dem Geschwindigkeitskoeffizienten $k_{\mathrm{t}}$ zusammengefasst. Damit ergibt sich für die Abnahme der Radikalkonzentration:

$$
\frac{\mathrm{d} c_{\mathrm{R}}}{\mathrm{d} t}=-2 \cdot k_{\mathrm{t}} \cdot c_{\mathrm{R}}^{2}
$$

Einen Ausdruck für den zeitlichen Verlauf der Radikalkonzentration nach einem Laserpuls erhält man durch Integration von Gleichung 3.4.

$$
c_{\mathrm{R}}=\left(2 \cdot k_{\mathrm{t}} \cdot t+\frac{1}{c_{\mathrm{R}}^{0}}\right)^{-1}
$$

Daraus folgt für die relative Monomerkonzentration $c_{\mathrm{M}}(t) / c_{\mathrm{M}}^{0}$ nach einem Laserpuls: 


$$
\frac{c_{\mathrm{M}}(t)}{c_{\mathrm{M}}^{0}}=\left(2 \cdot k_{\mathrm{t}} \cdot c_{\mathrm{R}}^{0} \cdot t+1\right)^{-k_{\mathrm{p}}} / 2 k_{\mathrm{t}}
$$

$c_{\mathrm{M}}^{0}$ : Monomerkonzentration vor Einstrahlung des Laserpulses

$c_{\mathrm{R}}^{0}$ : durch den Laserpuls erzeugte Startradikalkonzentration

Die im Experiment ermittelten Konzentrations-Zeit-Verläufe können mit Gleichung 3.6 angepasst werden, wenn man von einer idealen Polymerisationskinetik mit konstanten Geschwindigkeitskoeffizienten ausgeht bzw. man die Geschwindigkeitskoeffizienten als über die untersuchte Zeit gemittelte Geschwindigkeitskoeffizienten ansieht. Außerdem wird der Einfluss von Transferreaktionen (siehe Abschnitt 3.3) auf das Messsignal bei diesen Anpassungen nicht berücksichtigt.

In Kapitel 8 wird abweichend von idealer Kinetik (und Gleichung 3.6) die Abhängigkeit des Terminierungsgeschwindigkeitskoeffizienten $k_{\mathrm{t}}$ von der Kettenlänge der reagierenden Radikale diskutiert.

Aus der Anpassung erhält man nach Gleichung 3.6 die Parameter $k_{\mathrm{t}} \cdot c_{\mathrm{R}}^{0}$ und $k_{\mathrm{t}} / k_{\mathrm{p}}$. Bei Kenntnis von $c_{\mathrm{R}}^{0}$ oder $k_{\mathrm{p}}$ kann $k_{\mathrm{t}}$ bestimmt werden. Da $c_{\mathrm{R}}^{0}$ nicht direkt zugänglich ist, wird das bis zu hohen Monomerumsätzen konstante $k_{\mathrm{p}}$ in separaten Experimenten ermittelt und übernommen. $k_{\mathrm{p}}$ läßt sich aus der von Olaj entwickelten Pulsed Laser Polymerizations-Size Exclusion Chromatography (PLP-SEC) Technik absolut bestimmen [1, 2]. Allerdings wird auch für $k_{\mathrm{p}}$ eine Kettenlängenabhängigkeit diskutiert [3, 4].

Die Methode der Bestimmung von $k_{\mathrm{t}}$ über Gleichung 3.6 in Kombination mit der PLP-SECTechnik wurde bereits für zahlreiche Homo- und Copolymerisationen von Acrylaten und Methacrylaten durchgeführt [5, 6, 7, 8, 9].

\subsection{Umsatzabhängigkeit der Terminierungsreaktion}

Da sich im Verlauf einer radikalischen Polymerisation bis zu hohem Umsatz die Viskosität des Reaktionsmediums um mehrere Größenordungen ändert [10], übt die Diffusionsabhängigkeit der Geschwindigkeitskoeffizienten einen entscheidenden Einfluss auf die Reaktionskinetik aus. Von Buback [11] wurde ein Modell mit physikalisch begründeten Parametern entwickelt, das hier vorgestellt werden soll.

Die Änderung des Wachstumsgeschwindigkeitskoeffizienten $k_{\mathrm{p}}$ ist gering und $k_{\mathrm{p}}$ kann bis zu hohen Umsätzen als konstant angenommen werden [4]. 
$k_{\mathrm{p}}$ lässt sich unter Berücksichtigung von Diffusion durch folgenden Ansatz beschreiben:.

$$
\frac{1}{k_{\mathrm{p}}}=\frac{1}{k_{\mathrm{p}}^{0}}+\frac{1}{k_{\mathrm{p}, \mathrm{D}}}
$$

$k_{\mathrm{p}}{ }^{0}$ gibt dabei dem Wachstumskoeffizienten ohne Einfluss von Diffusion und $k_{\mathrm{p}, \mathrm{D}}$ den diffusionsabhängigen Beitrag von $k_{\mathrm{p}}$ an. Es wird angenommen, dass sich $k_{\mathrm{p}, \mathrm{D}}$ umgekehrt proportional zur Viskosität der Lösung verhält.

$$
k_{\mathrm{p}, \mathrm{D}}=\frac{k_{\mathrm{p}, \mathrm{D}}^{0}}{\eta_{\mathrm{r}}} \quad \text { mit } \eta_{\mathrm{r}}=\frac{\eta}{\eta_{0}}
$$

Dabei ist $\eta_{\mathrm{r}}$ die relative Viskosität, gegeben durch den Quotienten aus der Viskosität der Mischung $(\eta)$ und der Viskosität der Lösung bei $0 \%$ Monomerumsatz $\left(\eta_{0}\right)$.

Für $k_{\mathrm{p}}$ ergibt sich damit:

$$
\frac{1}{k_{\mathrm{p}}}=\frac{1}{k_{\mathrm{p}}^{0}}+\frac{\eta_{\mathrm{r}}}{k_{\mathrm{p}, \mathrm{D}}^{0}}
$$

Der Terminierungskoeffizient lässt sich nicht so einfach beschreiben. Er übersteigt den Wachstumskoeffizient um mehrere Größenordnungen und ist in der Regel von Beginn an diffusionskontrolliert. Die Terminierung lässt sich in drei aufeinander folgende Schritte unterteilen:

(I) Translationsdiffusion (TD)

(II) Segmentdiffusion (SD)

(III) Chemische Reaktion (CR)

(I) beschreibt die Bewegung zweier Radikale aufeinander zu, (II) die Ausrichtung der Radikalzentren und (III) die chemische Reaktion. Dieser Ansatz des durch Diffusion bestimmten Terminierungsgeschwindigkeitskoeffizienten $k_{\mathrm{t}, \mathrm{D}}$ [12] wird mit folgender Gleichung beschrieben:

$$
\frac{1}{k_{\mathrm{t}, \mathrm{D}}}=\frac{1}{k_{\mathrm{TD}}}+\frac{1}{k_{\mathrm{SD}}}+\frac{1}{k_{\mathrm{CR}}}
$$

Für $k_{\mathrm{TD}}$ wird eine umgekehrte Proportionalität zur Viskosität angenommen: 


$$
k_{\mathrm{TD}}=\frac{k_{\mathrm{TD}}^{0}}{\eta_{\mathrm{r}}}
$$

wobei $k_{\mathrm{TD}}{ }^{0}$ den translationsdiffusionskontrollierten Geschwindigkeitskoeffizienten bei $0 \%$ Umsatz bezeichnet.

Gleichung 3.10 gibt jedoch nicht den gesamten Verlauf von $k_{\mathrm{t}}$ mit dem Umsatz wieder. Bei hohen Umsätzen wird die Translation der Moleküle durch die hohe Viskosität stark eingeschränkt. Die sogenannte Reaktionsdiffusion wird bestimmend für die Terminierung [13]. Dabei erfolgt die Terminierung unter wesentlicher Mitwirkung von Wachstumsschritten, durch die sich die radikalischen Zentren noch unter Bedingungen annähern können, bei denen die Translationsdiffusion weitgehend eingeschränkt ist. Dieser Prozess ist proportional zum Kettenwachstum und der Monomerkonzetration $c_{\mathrm{M}}$.

$$
k_{\mathrm{t}, \mathrm{RD}}=C_{\mathrm{RD}} \cdot k_{\mathrm{p}} \cdot(1-X)
$$

wobei $C_{\mathrm{RD}}=$ Reaktionsdiffusionskonstante und für den Umsatz $X$ gilt:

$$
X=\frac{c_{\mathrm{M}}^{o}-c_{\mathrm{M}}}{c_{\mathrm{M}}^{o}}
$$

Die gesamte Terminierungsreaktion kann nun angegeben werden durch

$$
k_{\mathrm{t}}=k_{\mathrm{t}, \mathrm{D}}+k_{\mathrm{t}, \mathrm{RD}}
$$

Durch Einsetzten von Gleichung 3.9 in 3.10-3.13 ergibt sich unter der Bedingung, dass $k_{\mathrm{CR}}$ wesentlich größer als $k_{\mathrm{TD}}$ und $k_{\mathrm{SD}}$ ist:

$$
k_{\mathrm{t}}=\frac{1}{\frac{1}{k_{\mathrm{SD}}}+\frac{\eta_{\mathrm{r}}}{k_{\mathrm{TD}}^{0}}}+\frac{C_{\mathrm{RD}} \cdot(1-X)}{\frac{1}{k_{\mathrm{p}, 0}^{0}}+\frac{\eta_{\mathrm{r}}}{k_{\mathrm{p}, \mathrm{D}}^{0}}}
$$

Betrachtet man nur Reaktionsdiffusionsprozesse, so vereinfacht sich Gleichung 3.14 zu

$$
k_{\mathrm{t}}=k_{\mathrm{t}, \mathrm{RD}}=C_{\mathrm{RD}} \cdot k_{\mathrm{p}} \cdot(1-X)
$$

Gleichung 3.14 beschreibt die Terminierungsreaktion der radikalischen Polymerisation gut. Es können 2 Klassen von Monomeren unterschieden werden [14]. Monomere vom Typ A, für 
den Butylacrylat ein Beispiel ist, zeigen über einen größeren Umsatzbereich nur eine verhältnismäßig kleine Änderung von $k_{\mathrm{t}}$. Bei Monomeren dieser Klasse fällt $k_{\mathrm{t}}$ nach einem meist etwas steileren Anfangsbereich (bis etwa $10 \%$ ) bis zu sehr hohen Umsätzen mit kleiner Steigung gleichmäßig ab.

Methylmethacrylat ist neben Styrol einer der typischen Vertreter von Typ B Monomeren. Deren Verlauf von $k_{\mathrm{t}}$ gegen den Umsatz ist schematisch in Abb. 3.1 dargestellt.

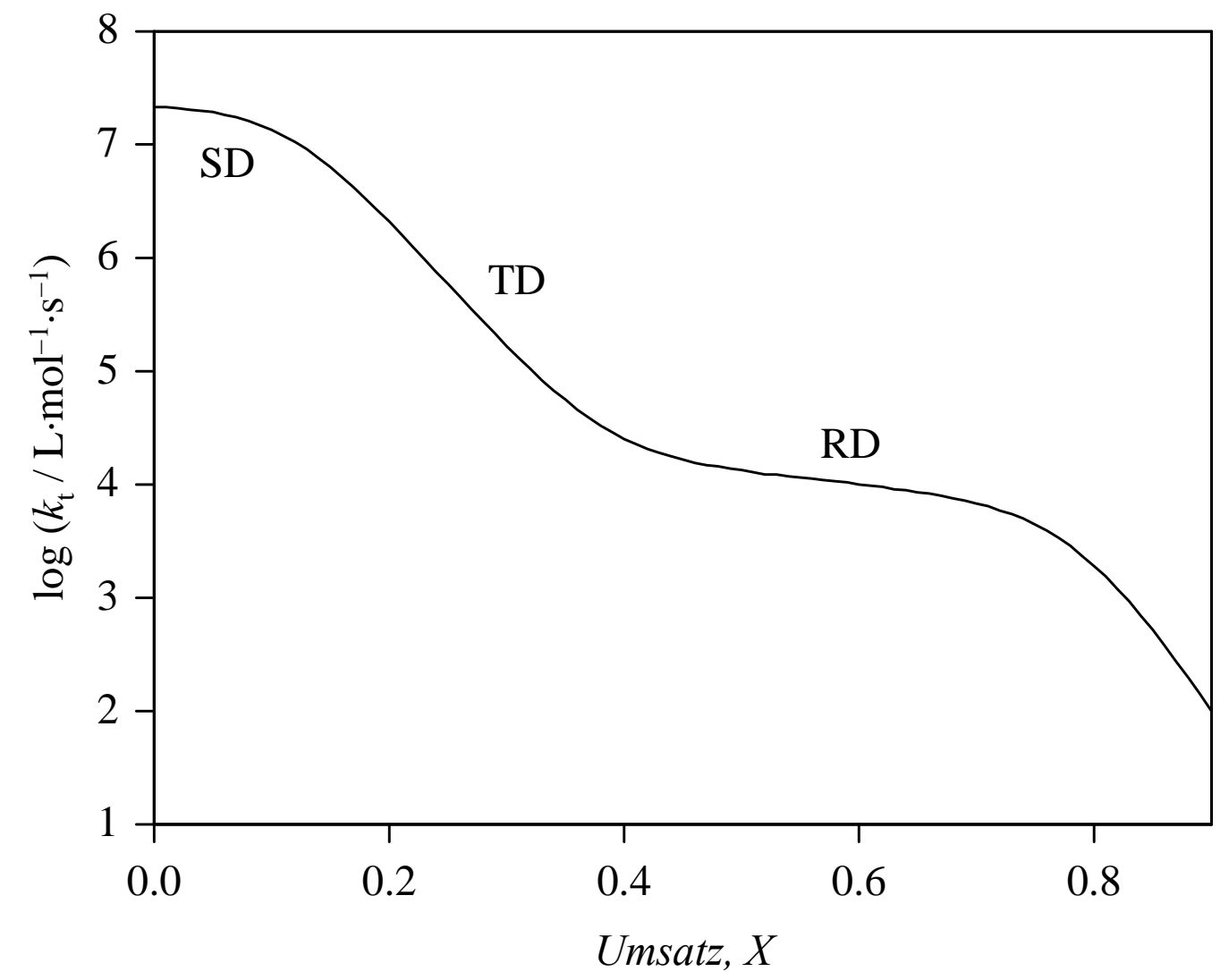

Abb. 3.1: Variation von $k_{\mathrm{t}}$ als Funktion des Umsatzes eines Monomers des Typs B.

Bei geringem Umsatz ist die Segmentdiffusion geschwindigkeitsbestimmend, erst ab einem gewissen Umsatz wird durch die Viskosität die Translation langsamer als die Segmentdiffusion. Während bei der Geschwindigkeitskontrolle durch Segmentdiffusion ein eher konstantes $k_{\mathrm{t}} \mathrm{zu}$ beobachten ist (Plateaubereich), sinkt der Geschwindigkeitskoeffizient im Bereich der Translationsdiffusionskontrolle um mehrere Größenordnungen, da die Translationsdiffusion umgekehrt proportional zur Viskosität ist. Mit weiter fortschreitendem Umsatz wird die Abnahme der Geschwindigkeit der Terminierungsreaktion teilweise durch Reaktionsdiffusion kompensiert, so dass der Verlauf von $k_{\mathrm{t}}$ mit dem Umsatz wieder abflacht. Bei hohen Umsätzen unterliegt schließlich auch $k_{\mathrm{p}}$ der Translationsdiffusionskontrolle und 
sinkt. Da $k_{\mathrm{t}}$ in diesem Bereich nach Gleichung 3.12 direkt von $k_{\mathrm{p}}$ abhängt, fällt auch der Wert von $k_{\mathrm{t}}$ erneut stärker ab.

Der Bereich der Translationsdiffusionskontrolle wird oft als Geleffekt oder NorrishTrommsdorff-Effekt [15, 16] bezeichnet. Im weiteren Verlauf dieser Arbeit wird die Bezeichnung Geleffekt verwendet. Der Beginn dieses Bereichs wird hervorgerufen durch eine bestimmte Konzentration an Polymer in der Lösung, ab der die Polymerradikale nicht mehr isoliert, sondern ineinander ,verstrickt“ vorliegen [17]. Ist diese Konzentration erreicht, tritt eine Beschleunigung der Polymerisationsgeschwindigkeit auf. Die kritische Konzentration hängt nicht allein vom Umsatz, sondern auch von Art und Größe der einzelnen Polymerketten ab. Neben diesem Erklärungsansatz gibt es in der Literatur noch weitere Vorschläge, z.B. kann das Einsetzen des Geleffekts auch als Übergang zwischen verdünnter und halbverdünnter Lösung beschrieben werden [18].

\subsection{Kettenübertragungsreaktionen}

Einige Substanzen erlauben die gezielte Herstellung von Polymer mit bestimmter Molmasse. Solche Stoffe werden Molmassenregler (engl. chain transfer agent) genannt. Sie erzeugen in dem unter 3.1 genannten Reaktionsschema einen zusätzlichen Schritt, bei dem die Radikalfunktion von der wachsenden Kette auf das Reglermolekül übertragen wird, das wiederum durch Reaktion mir einem Monomermolekül eine neue Kette startet. Man kann dabei zwischen nicht katalytischen und katalytischen Agenzien unterscheiden. Die nicht katalytischen sind nach der Reaktion als Endgruppe im Polymer zu finden. Das folgende Schema zeigt den Mechanismus der Kettenübertragung für den nicht katalytischen Fall von Dodecylmercaptan (DDM, H-SR).

$$
\begin{array}{ll}
\mathrm{R}_{\mathrm{i}} \cdot+\mathrm{M} \rightarrow \mathrm{R}_{\mathrm{i}+1} \cdot & k_{\mathrm{p}} \\
\mathrm{R}_{\mathrm{i}} \cdot+\mathrm{H}-\mathrm{SR} \rightarrow \mathrm{P}_{\mathrm{i}}+\cdot \mathrm{SR} & k_{\mathrm{tr}, \mathrm{HSR}} \\
\cdot \mathrm{SR}+\mathrm{M} \rightarrow \mathrm{R}_{1} \cdot & k_{\mathrm{p}, \mathrm{HSR}}
\end{array}
$$

Geschwindigkeitsbestimmend ist Reaktion (b). Der nachfolgende Wachstumsschritt verläuft schnell.

DDM ist eine gesättigte Verbindung. Die Thiolbindung ist jedoch homolytisch leicht zu spalten. Aus diesem Grund bewirkt DDM nicht nur eine Kettenübertragungsreaktion, sondern es kann auch als Initiator wirken [19, 20]. 
Transferreaktionen sind auch ohne Hinzufügen spezieller Reglermoleküle durch direktes Übertragen der Radikalfunktion auf ein Monomer- oder Polymermolekül mit allerdings kleineren Geschwindigkeitskoeffizienten möglich.

\subsection{Literatur Kapitel 3}

[1] O. F. Olaj, I. Bitai, F. Hinkelmann, Macromol. Chem. 1987, 188, 1689.

[2] O. F. Olaj, I. Schnöll-Bitai, Eur. Polym J. 1989, 25, 635.

[3] O. F. Olaj, P. Vana, M. Zoder, A. Kornherr, G. Zifferer, Macromol. Rapid. Commun. 2000, $21,913$.

[4] S. Beuermann, M. Buback, Prog. Polym. Sci. 2002, 27, 191.

[5] B. Degener, Dissertation, Göttingen 1992.

[6] C. H. Kurz, Dissertation, Göttingen 1995.

[7] M. Buback, C. Kowollik, Macromolecules 1998, 31, 3221.

[8] M. Buback, C. Kowollik, Macromolecules 1999, 32, 1445.

[9] M. Buback, C. Kowollik, C. H. Kurz, A. Wahl, Macromol. Chem. Phys. 2000, 201, 464.

[10] M. Buback, J. Schweer, Z. Phys. Chem. Neue Folge 1989, 161, 153.

[11] M. Buback, Macromol. Chem. 1990, 191, 1575.

[12] S. W. Benson, A. M. North, J. Am. Chem. Soc. 1962, 84, 935.

[13] G. V. Schulz, Z. Phys. Chem. 1956, 8, 290.

[14] M. Buback, B. Huckestein, G. T. Russell, Macromol. Chem. Phys. 1994, 195, 539.

[15] E. Trommsdorff, Makromol. Chem. 1947, 1, 169.

[16] R. G. W. Norrish, R. R. Smith, Nature 1942, 150, 336.

[17] J. N. Cardenas, K. F. O’Driscoll, J. Polym. Sci., Polym. Chem. Ed. 1976, 14, 883.

[18] K. Horie, I. Mita, H. Kambe, J. Polym. Sci., Part A, 1968, 6, 2663.

[19] V. El Rezzi, Dissertation, Göttingen 2001.

[20] R. A. Hutchinson, D. A. Paquet, J. H. McMinn, Macromolecules 1995, $28,5655$. 


\section{EXPERIMENTELLE TECHNIKEN}

In diesem Kapitel sollen die bei den Messungen verwendeten Geräte vorgestellt werden. Zuerst erfolgt eine Beschreibung der verwendeten Hochdruckzelle (4.1), der Druckerzeugung (4.2), sowie der Geräte der Temperaturregelung (4.3). Anschließend werden das zur Umsatzbestimmung verwendete IR/NIR-Spektrometer (4.4) und die verwendeten Detektoren (4.5) vorgestellt. Schließlich wird die Messanordungen für die SP-PLP-Experimente (4.6) und die Auswertung der Messdaten (4.7) sowie die GPC (4.8) beschrieben.

\subsection{Hochdruckmesszelle}

Spektroskopische Untersuchungen der laserinduzierten Polymerisation unter hohem Druck erfordern zur quantitativen Analyse eine optische Durchstrahlzelle, deren Fenster eine hohe Transparenz im Nahinfrarot-Bereich und bei der verwendeten Laserwellenlänge aufweisen und die eine ausreichende mechanische Festigkeit besitzen. Dabei muss das gesamte Probenvolumen mit dem Laser bestrahlt werden. Außerdem muss ein Kontakt der Probe mit den Zellwänden vermieden werden, um einen katalytischen Einfluss des Metalls auszuschließen.

Die verwendete Hochdruckmesszelle ist bis zu Drücken von 3500 bar und Temperaturen von $350{ }^{\circ} \mathrm{C}$ einsetzbar. Abbildung 4.1 zeigt ein Schnittbild durch eine Zelle. Der hohlzylindrische Zellkörper (Werkstoff RGT 601, Werkstoffnr. 2.4668, Arbed Saarstahl) hat eine Länge von $100 \mathrm{~mm}$, einen Außendurchmesser von $80 \mathrm{~mm}$ und einen Innendurchmesser von $22 \mathrm{~mm}$. Die Dichtung erfolgt durch konische Stempel St (Werkstoff RGT 12, Werkstoffnr. 2.4969, Arbed Saarstahl), die durch Flansche Fl (Werkstoffnr. 2.4668) mit sechs Schrauben S (Werkstoffnr. 2.4969) an den Zellkörper gepresst werden. Die Öffnungswinkel der Konen betragen $78^{\circ}$, die der Stempel $75^{\circ}$, so dass die Innenkante des Zellkörpers gegen die Fläche des Stempels dichtet. Durch die Auswahl von unterschiedlich langen Stempeln kann die optische Schichtlänge variiert werden (bis maximal $20 \mathrm{~mm}$ ). Die zentralen Bohrungen in den Stempeln haben einen Durchmesser von $10 \mathrm{~mm}$. 


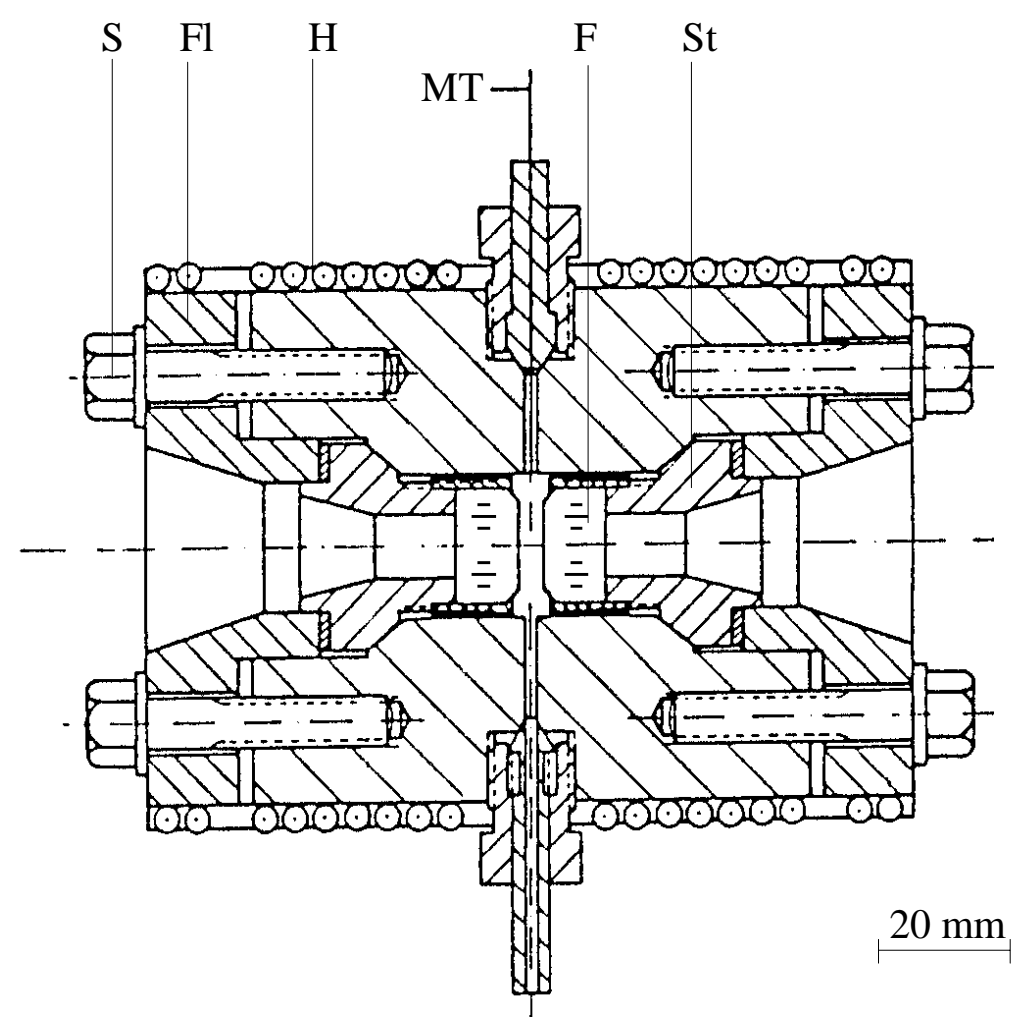
(S) Schraube
(F) Hochdruckfenster
(Fl) Flansch
(St) Stempel
(H) Heizmantel
(MT) Mantelthermoelement

Abb. 4.1: Optische Hochdruckmesszelle.

Zum Anschluss von Hochdruckkapillaren (Außendurchmesser 1/4 Zoll, Nova Swiss) und zur Einführung eines Thermoelements MT bis an die Messschicht stehen vier Bohrungen (1.5 mm Durchmesser) zur Verfügung. Nicht benötigte Kapillarbohrungen werden durch Blindstopfen verschlossen. Um die Zelle gut im Spektrometer justieren zu können, ist an einem Flansch eine Platte mit Schrauben befestigt, die an der oberen Kante in einen Holzgriff übergeht. Die Metallplatte ist in der Mitte durchbohrt und erlaubt so den Durchtritt der Strahlung.

Die optischen Fenster F bestehen aus synthetischem Saphireinkristall (Durchmesser 18 mm, Höhe $=10$ mm, UV grade, Roditi, Union Carbide), der nach dem Czochralski-Verfahren aus der Schmelze gezogen wird. Sie sind zwischen 200 und $5000 \mathrm{~nm}\left(2000 \mathrm{~cm}^{-1}\right.$ bis $\left.50000 \mathrm{~cm}^{-1}\right)$ optisch transparent. Die optische Durchlässigkeit im UV-Bereich bleibt bei Bestrahlung durch den Laser unverändert und ist auch bei verschiedenen Messtemperaturen jeweils konstant. Die beiderseits optisch polierten Fenster werden nach dem Poulter-Prinzip [1] gegen die ebenfalls optisch polierten (Mikrodiamant, Körnung 4 bis $8 \mu \mathrm{m}$ ) Auflageflächen der Stempel gedichtet 
und mit Überwurfkappen fixiert. Eine $12 \mu \mathrm{m}$ dicke Teflonfolie zwischen Saphir und Stempel dient zum Ausgleich von Oberflächenunebenheiten.

\section{Innenzelle}

Um eine vollständige Bestrahlung des gesamten Probenvolumens zu gewährleisten und um katalytische Einflüsse der Zellwände auszuschließen, wird die Polymerisation in einer in die Hochdruckzelle eingesetzten Innenzelle (IZ) durchgeführt (Abbildung 4.2). Sie besteht aus einem Teflonschlauch (Innendurchmesser $9 \mathrm{~mm}$, Außendurchmesser $10 \mathrm{~mm}$, Länge ca. $11.5 \mathrm{~mm}$ ), in den zwei optisch polierte Quarzfenster (Durchmesser $10 \mathrm{~mm}$, Höhe je $5 \mathrm{~mm}$, Heraeus Quarzglas, INFRA-SIL 301) eingesetzt werden. Diese sind im Bereich über $3000 \mathrm{~cm}^{-1}$ strahlungsdurchlässig.

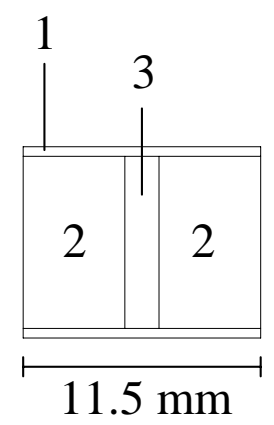

\footnotetext{
Abb. 4.2: Optische Innenzelle für SP-PLP Messungen in Substanz. (1) Teflonschlauch; (2) $\mathrm{SiO}_{2}$-Fenster; (3) Probenvolumen.
}

Um die Dichtigkeit zwischen Teflonschlauch und Fenster zu erhöhen, werden die zylindrischen Flächen der Fenster poliert (Mikrodiamant, Körnung 4-8 $\mu \mathrm{m}$ ). Vor dem Einsetzen der Fenster in den Teflonschlauch müssen die Schlauchenden etwas aufgeweitet werden. Die Innenzelle wird mit einem Teflonfüllkörper in der Mitte der Hochdruckzelle fixiert.

Für eine Übersicht über die hier beschriebenen Hochdrucktechniken siehe auch Ref. [2].

\subsection{Druckerzeugung}

Der schematische Aufbau der Druckerzeugungsanlage ist in Abbildung 4.3 wiedergegeben. Das als Druckmedium verwendete $n$-Heptan läuft von einem Vorratsgefäß VG in eine Spindelpresse S (Volumen $12 \mathrm{~cm}^{3}$ ), mit der es nach Schließen der Absperrventile V auf den gewünschten Druck komprimiert wird. 


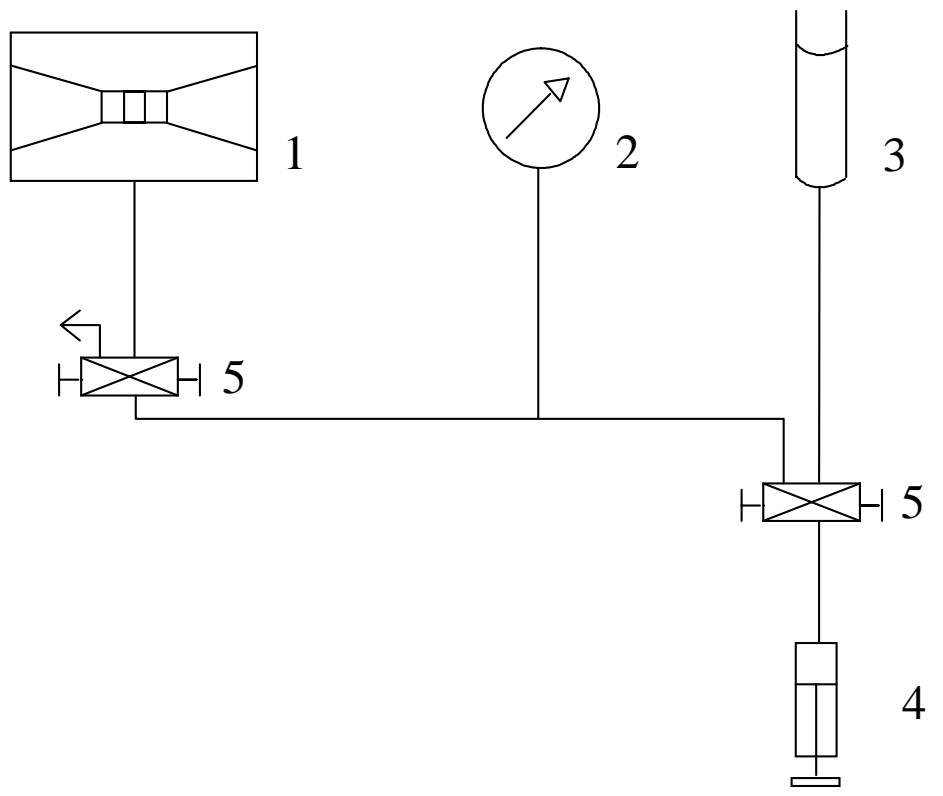

1: Hochdruckzelle

2: Manometer

3: Vorratsgefäß

4: Spindelpresse

5: Absperrventil

Abb. 4.3: Druckerzeugungsanlage.

\subsection{Temperaturregelung der optischen Hochdruckzellen}

Die Beheizung der Hochdruckzelle erfolgt über Widerstandsmantelheizleiter (CGE Alsthom). Sie sind Messingmatrizen eingelassen, die der Zelle angepasst sind. Die Temperatur wird mit einem Chromel-Alumel-Thermoelement (Philips) gemessen, welches in einem Blindstopfen hart eingelötet ist und direkt bis an die Messschicht reicht. Die Temperaturregelung erfolgt durch einen PID-Regler (Eurotherm 815).

\subsection{IR/NIR-Spektrometer}

Zur Aufnahme der Infrarot- und Nahinfrarotspektren steht ein Fouriertransform-Spektrometer (IFS 88, Bruker) zur Verfügung. Der Probenraum hat gegenüber der Standardausführung eine vergrößerte Abdeckung und eine wärmeableitende Zellhalterung. Er wird kontinuierlich mit wasser- und kohlendioxidarmer Pressluft gespült. Die optische Konfiguration kann ja nach gewünschtem Spektralbereich variiert werden. Die in dieser Arbeit durchgeführten Messungen erfolgen mit einer Wolfram-Halogen-Quarzlampe als Strahlungsquelle, einem InSb-Detektor und einem siliciumbeschichteten Calciumfluorid-Strahlteiler. 


\subsection{Detektoren}

Zur zeitaufgelösten Bestimmung der Konzentrationsänderung des Monomeren bei fester Wellenlänge nach einem Laserpuls stehen drei Infrarot- bzw. Nahinfrarot-Detektoren zur Verfügung:

a) InSb, 1.0 bis $5.5 \mu \mathrm{m}$, Infrared Associates, I-457-IS, $1 \mathrm{~mm} \times 1 \mathrm{~mm}, \mathrm{~N}_{2}$ gekühlt

b) InAs, 1.0 bis $3.6 \mu \mathrm{m}$, EG\&G Judson, M-204 A, $1 \mathrm{~mm} \times 1 \mathrm{~mm}, \mathrm{~N}_{2}$ gekühlt

c) InGaAs (Indium-Gallium-Arsenid), 1.0 bis $2.0 \mu \mathrm{m}$, Epitaxx, ETX-2000 TE, $2 \mathrm{~mm} \times 2 \mathrm{~mm}$, thermoelektrisch gekühlt.

Diese drei Detektoren arbeiten nach dem photovoltaischen Prinzip. Die spektrale Empfindlichkeit der Detektoren wird durch die Größe $D^{*}$ gekennzeichnet, die Detektivität einer aktiven Detektorfläche von $1 \mathrm{~cm}^{2}$ und einer Geräuschbandbreite von $1 \mathrm{~Hz}$, bezogen auf die Wellenlänge der größten Empfindlichkeit. $D^{*}$ wird in der Einheit $\mathrm{cm} \mathrm{Hz}^{0.5} \mathrm{~W}^{-1}$ angegeben. Je höher der $D^{*}$-Wert, desto schneller kann der Detektor bei fester Vergleichsempfindlichkeit und bei fester Vergleichssignalfrequenz messen. Aus der Fluktuation der eintreffenden Hintergrundphotonen ergibt sich eine Leistungsgrenze, die einen idealen photovoltaischen Detektor beschreibt.

Für diese Arbeit kommt zur Detektion des ersten Obertons der $\mathrm{CH}$-Streckschwingung bei etwa $6200 \mathrm{~cm}^{-1}$ der InSb-Detektor bei der FT-IR-Spekroskopie, der InAs-Detektor an der SPPLP-Anlage (s. 4.6) zum Einsatz. InAs-Detektoren zeichnen sich im gemessenen Wellenlängenbereich durch eine größere Detektivität aus und sind daher für zeitaufgelöste Messungen besser geeignet. InSb-Detektoren dagegen sind in einem größeren Wellenlängenbereich einsetzbar.

\subsection{Messanordnung für Experimente mit zeitaufgelöster Umsatzdetektion (SP-PLP)}

In Abbildung 4.4 ist die in dieser Arbeit verwendete Messanordnung schematisch dargestellt [siehe auch 3, 4].

Die zur Initiierung der Polymerisation verwendeten Lichtpulse eines Excimerlasers (Lextra 50, Lambda Physik) werden mit Hilfe IR-durchlässiger UV-Spiegel S (INFRASIL, ZnSe-beschichtet) auf die optische Achse der Messzelle M gelenkt. Dabei dient ein zu 
eichender Detektor D vor der Messzelle zur Bestimmung der Energie der Laserpulse. Der Fotoverschluß FV direkt hinter dem Detektor dient zum Abfangen von Testlaserpulsen. Er wird wie der Laser vom Rechner PC gesteuert.

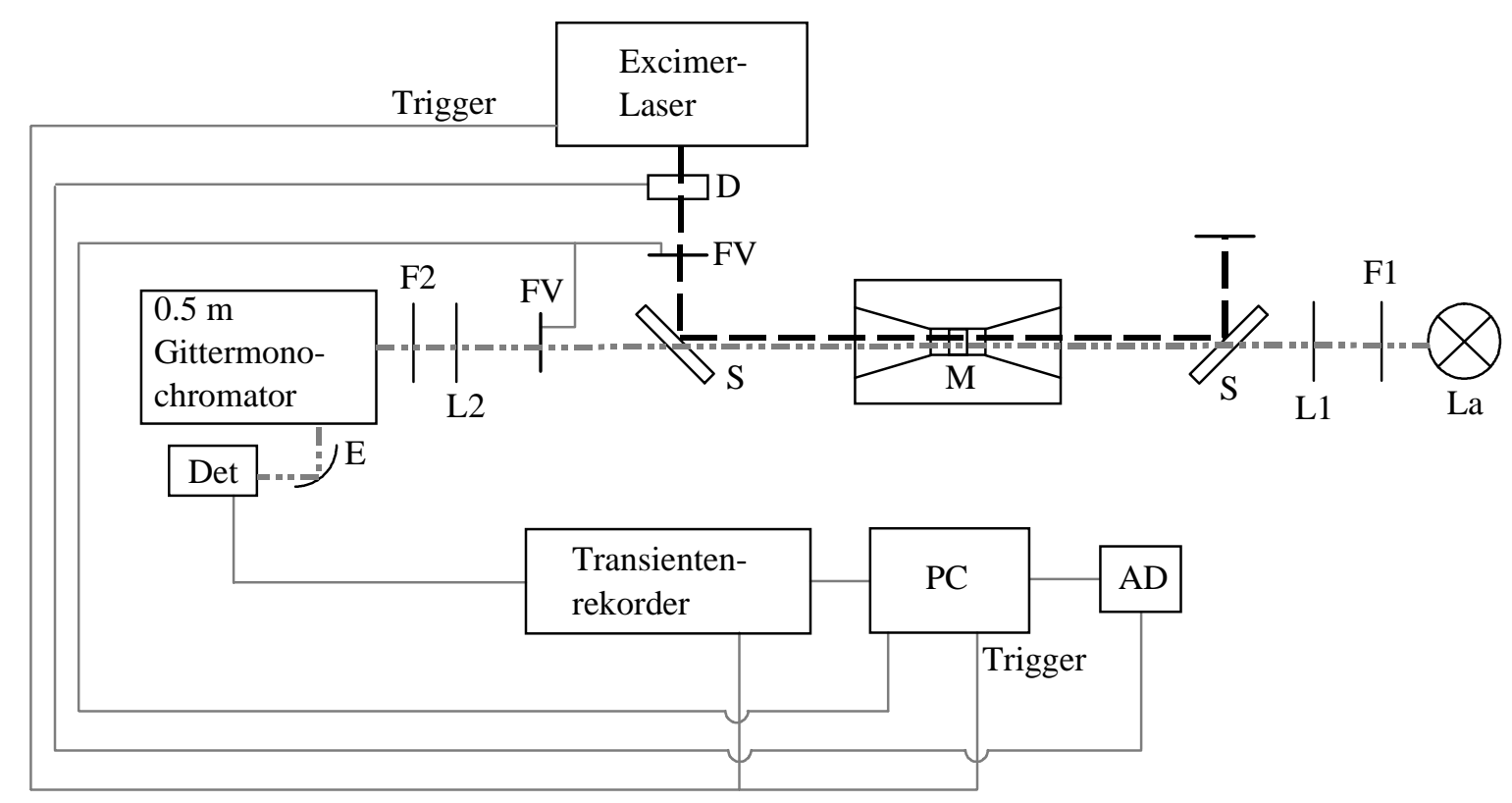
FV Fotoverschluß
D UV-Energiedetektor
M Messzelle
La Analysenlampe
- - - Strahlengang Laser
F Filter
Det NIR-Detektor (InAs)
S IR-durchlässiger
UV-Spiegel
E Ellipsoidalspiegel
- Strahlengang Analysenlicht
L Linsen

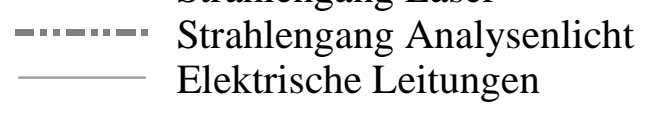

Abb. 4.4: Messanordnung für das SP-PLP-Experiment.

Die Abnahme der Monomerkonzentration wird über die NIR-Absorption verfolgt, indem man die Änderung der Absorbanz einer dem Monomer zugeordneten Schwingung detektiert. Dazu wird das Absorbtionsmaximum im Bereich des ersten Obertons der $\mathrm{CH}$-Streckschwingung an der Doppelbindung bei ca. $6170 \mathrm{~cm}^{-1}$ verwendet.

Als Strahlungsquelle dient eine Wolfram-Halogen-Quarzlampe La (General Electric 75 W), deren Betriebsspannung von einem Bleiakkumulator (12 V, $170 \mathrm{~A} / \mathrm{h})$ geliefert wird, um ein möglichst rauscharmes Signal zu erhalten. Um eine Initiierung der Polymerisation durch das Analysenlicht zu vermeiden, wird ein UV-Sperrfilter F1 (RG 695) verwendet. Durch die Linse $\mathrm{L} 1\left(\mathrm{CaF}_{2}, f=100 \mathrm{~mm}, d=50 \mathrm{~mm}\right)$ wird die Glühwendel der Lampe auf die Probe und durch die Linse L2 $\left(\mathrm{CaF}_{2}, f=100 \mathrm{~mm}, d=50 \mathrm{~mm}\right)$ auf den Eingangsspalt des Gittermonochromators (B\&M Spektronik, BM 50, 0.5 m, f/6.9) abgebildet. Das Analysenlicht wird danach an einem Interferenzgitter (Bausch\& Lomb, $76 \mathrm{~mm} \times 76 \mathrm{~mm}$, 600 1/mm, Blaze 
$1.6 \mathrm{~mm}$ bzw. 28041', $D=4.1 \mathrm{~nm} / \mathrm{mm}$ ) gebeugt und über einen Ellipsoidal-Spiegel E (Bruker Analytische Messtechnik $\mathrm{GmbH}, \mathrm{f} / 6, f_{1}=200 \mathrm{~mm}, f_{2}=40 \mathrm{~mm}$ ) auf einen mit flüssigem Stichstoff gekühlten InAs (Indium-Arsenid)-Detektor reflektiert. Ein Siliciumfilter F2 (Oriel, $1 \mathrm{~mm}, 1.05 \mu \mathrm{m}$, Transmission bei $9000 \mathrm{~cm}^{-1}-5000 \mathrm{~cm}^{-1}$ ) vor dem Monochromator lässt nur einen Ausschnitt des Analysenlichts passieren, so dass nur Licht einer Gitterordnung auf den Detektor fällt. Das analoge Detektorsignal wird mit einer Transientenrekorderkarte (16 bit TR 1621-4, Fast ComTec) aufgezeichnet und mit einem Computer verarbeitet.

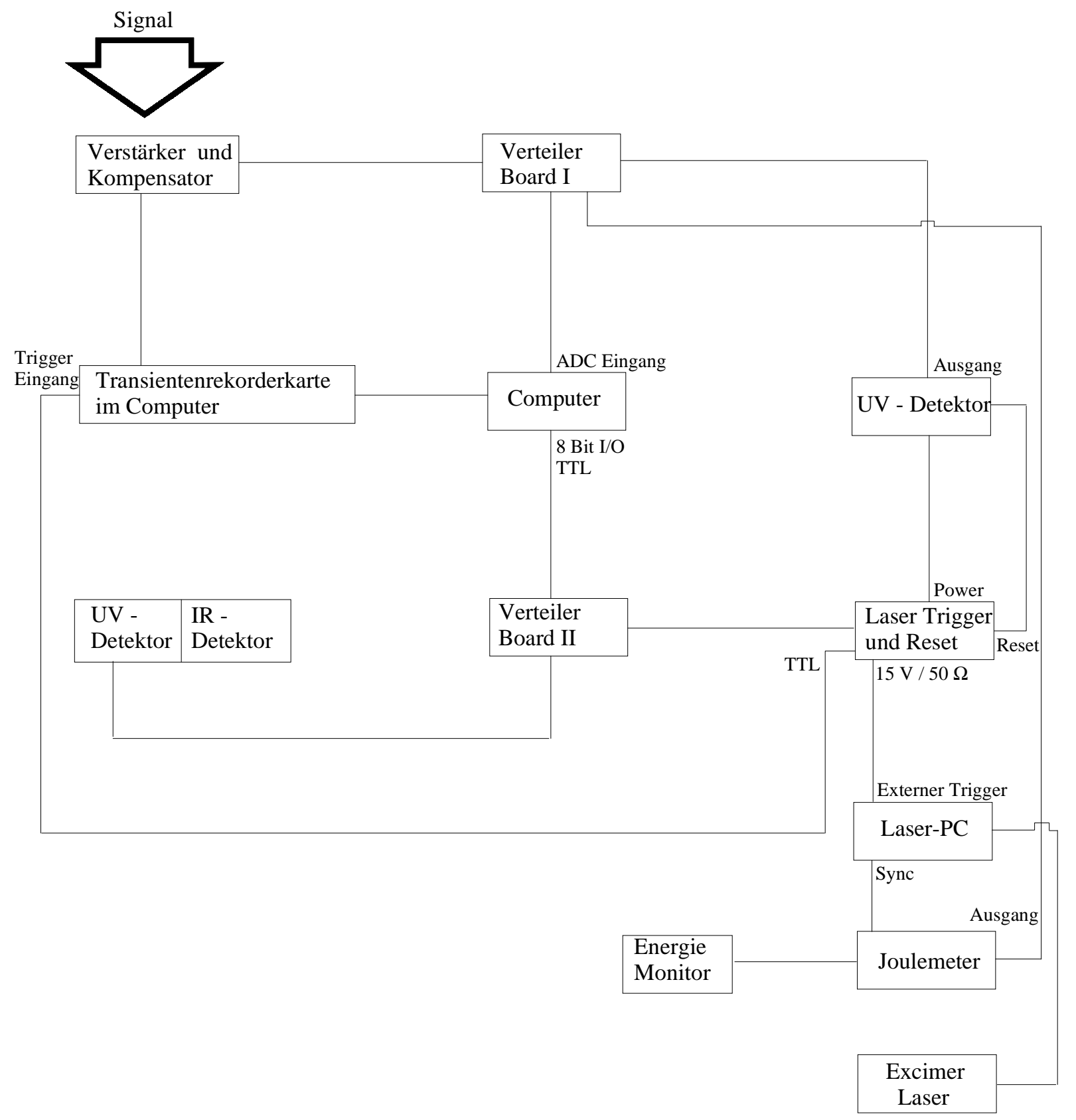

Abb. 4.5: Elektronischer Aufbau der SP-PLP-Anlage. 
Um störende Einflüsse durch Gebäudeschwingungen zu vermeiden, ist die Apparatur auf einer auf Gummischläuchen gelagerten Granitplatte montiert.

Der elektronische Aufbau der Anlage wurde bereits von Kowollik beschrieben [5] und ist in Abbildung 4.5 dargestellt. Im Rahmen dieser Arbeit wurde jedoch der bislang verwendete 12 bit Transientenrekorder (Rene Maurer AG, ADAM TC 210-1) durch die oben erwähnte 16 bit Transientenrekorderkarte ersetzt. Diese Karte erlaubt einerseits ein genaueres Einlesen der Messsignale und verfügt andererseits über einen deutlich größeren Speicher, wodurch pro Signal mehr Datenpunkte aufgenommen werden können.

Der Einbau der Transientenrekorderkarte erforderte das Erstellen von neuen Computerprogrammen zur Steuerung der Messapparatur und zur Auswertung der Messsignale. Diese Programme sind im Anhang zusammen mit einer aktuellen Betriebsanleitung abgedruckt.

\subsection{Auswertung der Messsignale}

Bei den zeitaufgelösten Messungen der Homo- und Copolymerisationen interessiert einerseits der Zeit-Umsatz Verlauf nach jedem Laserpuls und andererseits der jeweilige Gesamtumsatz. Die am Ende der Pulspakete erreichten Umsätze werden mit den aus den NIR-Spektren ermittelten Umsätzen als Kontrolle verglichen.

Zur Auswertung wird die Bande der CH-Streckschwingung am ungesättigten Kohlenstoffatom im Bereich des ersten Obertons herangezogen. Diese Bande tritt im Nahinfrarot-Bereich bei etwa $6170 \mathrm{~cm}^{-1}$ auf. Die Grundlage der Auswertung bildet das Lambert-Beersche Gesetz, dessen Gültigkeit über den gesamten Zustandsbereich erfüllt sein muss. Es stellt einen Zusammenhang zwischen der Absorbanz $A$ bei der untersuchten Wellenzahl und der Monomerkonzentration $c_{\mathrm{M}}$ her:

$$
A(v)=\log \left(I_{0} / I\right)=\varepsilon(v) \cdot c_{\mathrm{M}} \cdot d
$$

Dabei entspricht $\varepsilon$ dem molaren dekadischen Absorptionskoeffizienten, $d$ der Schichtlänge der Probe, $I_{0}$ der Intensität des auf die Probe auftreffenden und $I$ der Intensität des aus der Probe austretenden Lichts.

Im Gegensatz zum molaren dekadischen Absorptionskoeffizienten, der eine starke Druck- und Temperaturabhängigkeit aufweist, sind die Integrale der Banden bestimmter Schwingungen von diesen Parametern unabhängig. Die Bestimmung des Gesamtumsatzes $U(t)$ erfolgt deshalb unter Zuhilfenahme des integralen Lambert-Beerschen Gesetzes: 


$$
1-U(t)=\frac{c_{\mathrm{M}}}{c_{\mathrm{M}}^{0}}=\frac{\operatorname{Int}(t)}{\operatorname{Int}^{0}}
$$

mit:

$$
\operatorname{Int}=\int A(v) \mathrm{d} v=B \cdot c \cdot d
$$

$B$ : integrale Molarabsorptivität

Dabei wird die integrale Absorbanz (Int) der Bande bei etwa $6170 \mathrm{~cm}^{-1}$ berechnet (vgl. Abbildung 6.1). Für die Integration dieser Banden wird eine Horizontale durch den Absorbanzpunkt bei $6300 \mathrm{~cm}^{-1}$ als willkürlicher Nullpunkt gewählt, wobei die Absorbanz an dieser Stelle auch tatsächlich nahe Null ist.

Im Falle der Copolymerisation wird die Absorbanz der beiden Monomere addiert.

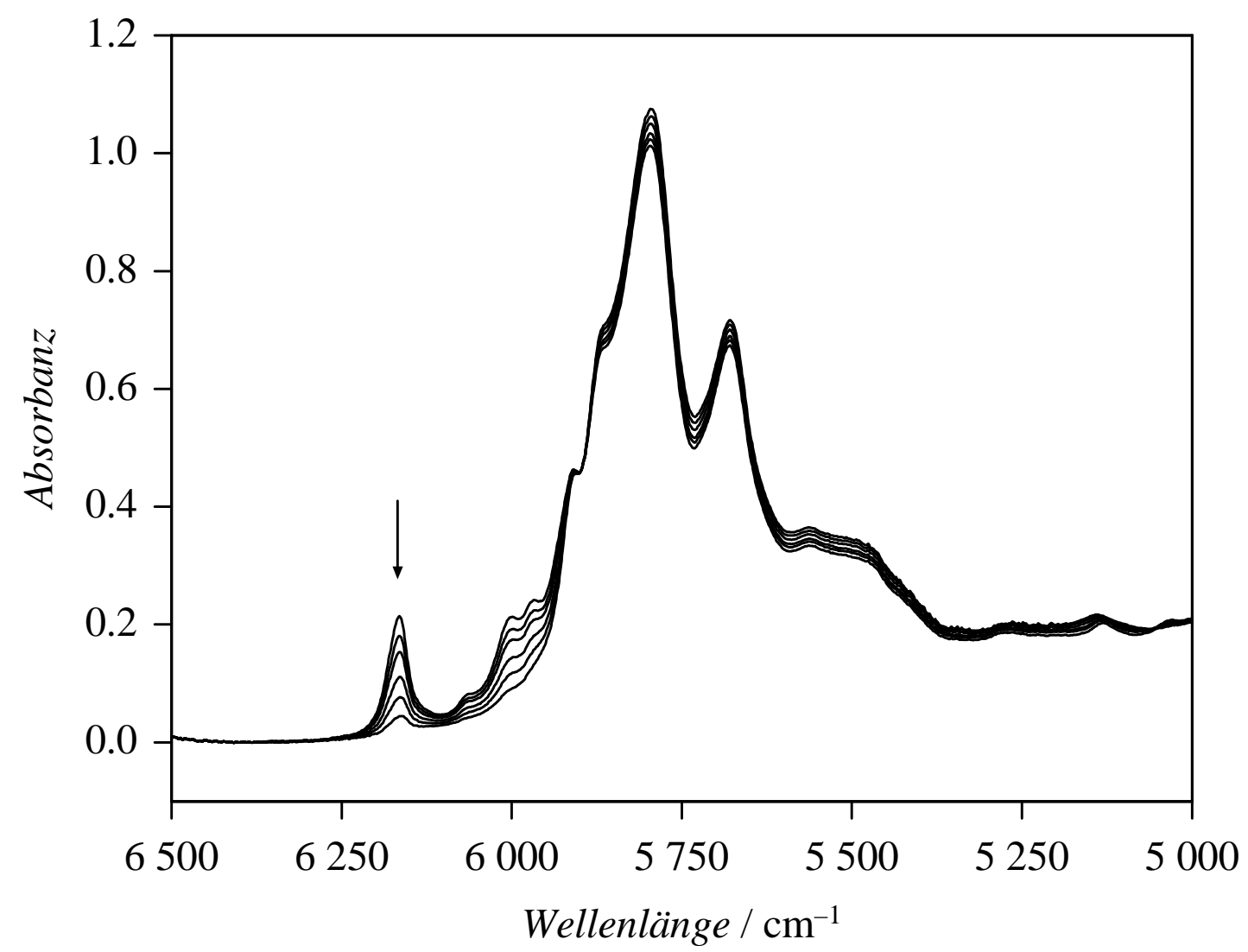

Abb. 4.6: Während der Copolymerisation einer MMA-DA-Mischung mit 11 mol \% MMA zwischen 0 und $72 \%$ Umsatz gemessene NIR-Spektren bei $40{ }^{\circ} \mathrm{C}, 1000$ bar. Die zur quantitativen Analyse verwendete Bande ist durch einen Pfeil gekennzeichnet. 
In Abb. 4.6 ist eine Serie von IR-Spektren im relevanten Wellenlängenbereich dargestellt, die bei einer Copolymerisation von MMA-DA mit 11 mol \% MMA in der Ausgangsmonomermischung zwischen 0 und $72 \%$ Umsatz gemessen wurden. Die zur Auswertung genutzte Bande ist durch einen Pfeil gekennzeichnet.

Bei der Anlage mit zeitaufgelöster Umsatzdetektion wird nach jedem Laserpuls die Änderung der Intensität des durch die Messzelle auf den Detektor auftreffenden Analysenlichts registriert. Mit Gleichung 4.1 kann daraus nach folgender Umformung die zeitliche Änderung der Monomerkonzentration berechnet werden:

$$
\Delta c_{\mathrm{M}}(t)=c_{\mathrm{M}}(t)-c_{\mathrm{M}}^{0}=\frac{\Delta A}{\varepsilon \cdot d}=\frac{\log \left(\frac{I_{0}}{I(t)}\right)}{\varepsilon \cdot d}
$$

Aus dieser zeitaufgelösten Messung des Monomer-Umsatzes lassen sich nun detaillierte Erkenntnisse über $k_{\mathrm{p}} / k_{\mathrm{t}}$ und somit über den Geschwindigkeitskoeffizienten der Terminierung gewinnen. Dazu werden Gleichungen für $c_{\mathrm{M}}(t) / c_{\mathrm{M}}^{0}$, die sich aus der Polymerisationskinetik ergeben, an die Messsignale über ein Iterationsverfahren (Levenberg-Marquardt-Algorithmus $[6,7]$ angepasst. Eine weitere Möglichkeit ist, das Differentialgleichungssystem des zugrundeliegenden Reaktionsschemas numerisch zu lösen.

Es werden außerdem zur Verbesserung der Anpassbarkeit der Messkurven an die ideale Polymerisationskinetik mehrere Einzelsignale coaddiert, wobei der Gesamtumsatz unter $10 \%$ liegt. Das Signal-Rausch-Verhältnis verbessert sich dabei mit der Quadratwurzel aus der Anzahl der Coadditionen.

Der bei den Messsignalen auftretende Umsatz pro Puls ist gering $(<1 \%)$, und die Konzentrationsänderung muss in sehr kurzer Zeit erfasst werden. Bei diesen Anforderungen an Empfindlichkeit und Geschwindigkeit in der Messtechnik ist es nicht möglich, einen größeren Wellenzahlenbereich gleichzeitig $\mathrm{zu}$ erfassen. Deshalb wird als Analysenlichtwellenlänge wiederum das Maximum der Bande des ersten Obertons der $\mathrm{CH}$ Steckschwingung an der Doppelbindung benutzt. Aufgrund der oben erwähnten Bandenverschiebung muss vor der Messung einer neuen Monomermischung mit dem Monochromator die neue Wellenlänge des Maximums selektiert werden.

Es ist jedoch zu beachten, dass das auf den Detektor fallende Licht wegen der Dispersion am Interferenzgitter des Monochromators nicht streng monochromatisch ist. Daher muss in Gleichung 4.4 der effektive molare Extinktionskoeffizient $\varepsilon_{\text {eff }}$ eingesetzt werden: 


$$
\varepsilon_{\mathrm{eff}}(\Delta v)=\frac{A_{\mathrm{eff}}}{c_{\mathrm{M}} \cdot d}
$$

$A_{\text {eff }}$ wird aus dem arithmetischen Mittel der Absorbanzen im Wellenzahlbereich $\Delta v$ bestimmt.

\subsection{Gelpermeationschromatographie (GPC)}

Die Bestimmung von Molmassen erfolgt mit der GPC (Gel-Permeations-Chromatographie, engl. Size-Exclusion-Chromatography (SEC)). Dabei wird eine Apparatur von Waters verwendet (Pump 515, DRI Detektor Model 410 mit 5 mm Säulen, Porengröße 10 $, 10^{3}, 10^{2}$ Å). Als Lösungsmittel kommt THF mit einem Fluss von $1 \mathrm{~mL} / \mathrm{min}$ bei $30{ }^{\circ} \mathrm{C}$ zum Einsatz. Die Datenaufnahme sowie die Verarbeitung der Daten erfolgt mit der von PSS (Mainz) gelieferten Software WinGPC 4. Zur Eichung der Daten wird ein Polymethylmethacrylatstandart (PSS) mit Molmassen von $4 \cdot 10^{2}$ bis $2.18 \cdot 10^{6} \mathrm{~g} / \mathrm{mol}$ verwendet.

\subsection{Literatur Kapitel 4}

[1] T.C. Poulter, Phys. Rev. 1932, 40, 860.

[2] M. Buback, C. Hinton, in High-pressure Techniques in Chemistry and Physics - A practical approach (Eds. B. W. Holzapfel and N. S. Isaacs), Oxford University Press: Oxford, 1997.

[3] M. Buback, H. Hippler, J. Schweer, H.-P. Vögele, Makromol. Chem. Rapid Commun. 1986, 7, 261.

[4] J. Schweer, Dissertation, Göttingen 1988.

[5] C. Kowollik, Dissertation, Göttingen 1999.

[6] Bevington, Data Reduction and Error Analysis for Physical Science, McGraw Hill, New York, 1969.

[7] Flammery, Teukolsky, Vetterling, Numerical Recepies, Cambridge University Press, Cambridge, 1989. 


\section{DURCHFÜHRUNG DER EXPERIMENTE}

\subsection{Vorbereitung der Substanzen}

\section{Monomere}

Die Acrylate $\left(R_{2}=H\right)$ und Methacrylate $\left(R_{2}=\right.$ Methyl $)$ haben die folgende allgemeine Struktur:<smiles>[R]OC(=O)C([R2])=C</smiles>

\section{Methylacrylat und Methylmethacrylat}

Methylacrylat (MA) (Fluka, purum (99.5\%)) und Methylmethacrylat (MMA) (Fluka, 99 \%) werden zur Entfernung des Stabilisators Hydrochinonmethylether über wasserfreiem Kaliumcarbonat mittels einer Vigreuxkolonne destilliert. Dabei darf das Monomer nicht mit Schlifffett in Berührung kommen, da dadurch eine Initiierung der Polymerisation erfolgen kann. Das gilt für alle im Folgenden aufgeführten Monomere.

\section{Butylacrylat und Butylmethacrylat}

Die Reinigung des Butylacrylats (BA) (Fluka, purum > $99 \%$ ) und des Butylmethacrylats (BMA) (Fluka, purum > $99 \%$ ) erfolgt ebenfalls mittels Destillation über $\mathrm{K}_{2} \mathrm{CO}_{3}$.

\section{Dodecylacrylat und Dodecylmethacrylat}

Bei dem in dieser Arbeit verwendeten Dodecylacrylat (DA) (Fluka, techn., stabilisiert mit Hydrochinonmethylether) handelt es sich eigentlich um ein Gemisch aus $55 \%$ Dodecylacrylat und $45 \%$ Tetradecylacrylat. DA und Dodecylmethacrylats (DMA) (Aldrich, 99\%) werden ebenfalls über $\mathrm{K}_{2} \mathrm{CO}_{3}$ destilliert, allerdings ohne Vigreuxkolonne, da der Vakuumverlust innerhalb der Kolonne zu groß ist.

\section{Isodecylacrylat}

Isodecylacrylat (Polysciences) wird durch Destillation mit einer Vigreuxkolonne über $\mathrm{K}_{2} \mathrm{CO}_{3}$ gereinigt. 


\section{Ethylhexylacrylat}

Ethylhexylacrylat (Fluka, $\geq 98 \%$ ) wird durch Destillation mit einer Vigreuxkolonne über $\mathrm{K}_{2} \mathrm{CO}_{3}$ gereinigt.

\section{Hexylacrylat und Cyclohexylacrylat}

Hexylacrylat (Lancaster, $95 \%$ ) und Cyclohexylacrylat (Lancaster, $98 \%$ ) werden durch Destillation mit einer Vigreuxkolonne über $\mathrm{K}_{2} \mathrm{CO}_{3}$ gereinigt.

\section{tert-Butylacrylat}

tert-Butylacrylat (Fluka, $\geq 98 \%$ ) wird durch Destillation mit einer Vigreuxkolonne über $\mathrm{K}_{2} \mathrm{CO}_{3}$ gereinigt.

\section{Benzylacrylat und Phenylacrylat}

Benzylacrylat (Lancaster, $97 \%$ ) und Phenylacrylat (Lancaster, 98+ \%) werden durch Destillation mit einer Vigreuxkolonne über $\mathrm{K}_{2} \mathrm{CO}_{3}$ gereinigt.

Die gereinigten Monomere werden bei $-10{ }^{\circ} \mathrm{C}$ gelagert. Direkt vor der Messung werden sie oder die vorher eingewogenen Monomermischungen durch mehrere Einfrier-Entgase-AuftauZyklen an einer Turbomolekularpumpe (Edwards E2M-1.5) vom Sauerstoff befreit.

\section{Initiatoren}

\section{2,2-Dimethoxy-2-phenylacetophenon}<smiles>COC(OC)(C(=O)c1ccccc1)c1ccccc1</smiles>

Der Photoinitiator 2,2-Dimethoxy-2-phenylacetophenon (DMPA) (Aldrich, $99 \%$ ) wird ohne weitere Reinigung eingesetzt. 
$\alpha$-Methyl-4-(methylmercapto)- $\alpha$-morpholinopropiophenon<smiles>CSc1ccc(C(=O)C(C)(C)N2CCOCC2)cc1</smiles>

$\alpha$-Methyl-4-(methylmercapto)- $\alpha$-morpholinopropiophenon (MMMP) (Aldrich, > $98 \%$ ) wird ohne weitere Reinigung eingesetzt.

\section{Azo-bis-isobutyronitril}<smiles>CC(C)(C#N)N=NC(C)(C)C#N</smiles>

Azo-bis-isobutyronitril (AIBN) (Merck, > $98 \%$ ) für die thermische Initiierung wurde durch Umkristallisation aus Diisopropylether gereinigt.

\section{tert-Amylperpivalat}<smiles>CCC(C)(C)OOC(=O)C(C)(C)C</smiles>

tert-Amylperpivalat (Akzo Nobel, > $98 \%$ ) wurde ohne weitere Reinigung eingesetzt.

Sonstige verwendete Substanzen

n-Dodecylmercaptan

$\mathrm{HS}$

Das Kettenübertragungsreagenz (DDM) (Fluka, > 97\%) wird ohne weitere Reinigung eingesetzt. 


\subsection{Auswahl der Messbedingungen}

\subsubsection{Messbedingungen der zeitaufgelösten Messung}

Mittels der SP-PLP-Technik sollten in dieser Arbeit Untersuchungen zur Bestimmung von $k_{\mathrm{t}}$ von MMA bei hohen Umsätzen, zur Kettenlängenabhängigkeit von $k_{\mathrm{t}}$ für verschiedene Acrylate und Methacrylate sowie $\mathrm{zu} k_{\mathrm{t}}$ in Copolymerisationen von Acrylat-Methacrylat Systemen durchgeführt werden. Für die Auswahl der Messbedingungen ist das Signal-RauschVerhältnis ausschlaggebend, allerdings muss das Auftreten unerwünschter Nebenreaktionen wie einer thermischen Untergrundreaktion vermieden werden.

Das Signal-Rausch-Verhältnis bessert sich mit zunehmendem Druck und zunehmender Temperatur, weil so der Umsatz pro Laserpuls steigt. Für alle SP-PLP-Experimente wurde eine Temperatur von $40{ }^{\circ} \mathrm{C}$ gewählt. Diese Temperatur ergab sich für die Untersuchungen zur MMA Homopolymerisation daraus, dass bei $40{ }^{\circ} \mathrm{C}$ die teilweise zur Vorpolymerisation von MMA genutzten Initiatoren AIBN und tert-Amylperpivalat nur sehr langsam zerfallen und so keine zusätzliche Polymerisation stattfindet. Außerdem waren Vergleichsdaten für niedrige Umsätze für $40^{\circ} \mathrm{C}$ aus der Literatur [1] verfügbar.

Für die Untersuchungen zur Kettenlängenabhängigkeit von $k_{\mathrm{t}}$ bot sich $40{ }^{\circ} \mathrm{C}$ als Reaktionstemperatur an, weil zu diesem Gesichtspunkt der Terminierungsreaktion bereits Ergebnisse für niedrige Monomerumsätze von Kowollik [1] bei dieser Temperatur existieren und somit die Vergleichbarkeit der Daten erleichtert wird.

Bei den Copolymerisationen wurde eine Temperatur von $40{ }^{\circ} \mathrm{C}$ wegen den ebenfalls bei dieser Temperatur bestimmten Daten der zugehörigen Homopolymerisationen gewählt.

Als Reaktionsdruck wurde für die meisten Messungen 1000 bar gewählt. Für viele Systeme wurden bei diesem Druck bereits Untersuchungen durchgeführt. Für niedrigere Drücke verschlechtert sich die Signalqualität deutlich. Der optimale Druck hängt jedoch auch mit der Fragestellung des Experiments zusammen. So konnte MMA durch die zunehmend bessere Signalqualität mit einsetzendem Geleffekt bei höheren Umsätzen auch unterhalb von 750 bar gemessen werden. Andererseits war für die Bestimmung der Kettenlängenabhängigkeit von $k_{\mathrm{t}}$ für MMA ein Druck von 2000 bar aufgrund der besseren Signalqualität vorzuziehen.

Bei der Wahl der optischen Schichtlänge in der Innenzelle ist darauf zu achten, dass die Absorbanz in dem für die quantitative spektroskopische Auswertung genutzten Spektralbereich um $6170 \mathrm{~cm}^{-1}$ in einem gut messbaren Bereich liegt. Wegen der Nichtlinearität des NIR-Detektors bei höheren Absorbanzen sollte das Maximum der zur Auswertung benutzen Bande 0.8 Absorbanzeinheiten nicht überschreiten. Andererseits führt 
eine zu kleine Schichtlänge zu größeren relativen Fehlern in der Auswertung. Eine gute Auswertbarkeit war bei Schichtlängen um $1.4 \mathrm{~mm}$ gegeben

Für die Anregung der Photoinitiatoren DMPA und MMMP eignet sich Laserlicht mit einer Wellenlänge von $351 \mathrm{~nm}$. Die Initiatoren haben bei dieser Wellenlänge eine hohe Absorbanz, die Monomere absorbieren dagegen fast nicht. Das als Druckmedium verwendete Heptan zeigt ebenfalls keine Absorbanz in diesem Bereich [2].

Es wurde bei einer weitgehend konstanten Laserenergie von $2.5 \mathrm{~mJ}$ pro Puls und Initiatorkonzentration von $5 \cdot 10^{-3}$ bis $1 \cdot 10^{-2} \mathrm{~mol} \cdot \mathrm{L}^{-1}$ gemessen.

\subsubsection{Vorpolymerisation}

Ein Ziel dieser Arbeit war es, die Terminierungsreaktion bei der Polymerisation von MMA bis $\mathrm{zu}$ hohen Monomerumsätzen zu untersuchen. Aufgrund des geringen Umsatzes pro Laserpuls ist der Verbrauch an Photoinitiator bei SP-PLP-Messungen von MMA im Plateaubereich sehr hoch. Somit ist die Photinitiatorkonzentration vor Erreichen höherer Umsätze zu gering für erfolgreiche SP-PLP Experimente. Es wurde deshalb der Ansatz verfolgt, durch eine thermische Reaktion ohne Verbrauch an Photoinitiator einen gewissen Umsatz zu erreichen und erst anschließend mit den eigentlichen SP-PLP-Messungen zu beginnen. Bei dieser „Vorpolymerisation“ war auf einige Bedingungen zu achten. Zum einen musste ein möglichst genau reproduzierbarer Startpunkt für die Einzelpulsexperimente eingestellt werden, weil schon kleine Änderungen die weitere Messung stark beeinflussen und damit die Vergleichbarkeit verschiedener Messreihen erschweren können. Zum anderen sollte das während der Vorpolymerisation erzeugte Polymer bezüglich seiner Zusammensetzung den Proben aus den SP-PLP-Experimenten ähnlich sein.

Bei thermischen Polymerisationen lässt sich durch Abkühlen und damit verbundenes Abbrechen der Reaktion ein gewünschter Umsatz erreichen. Dabei kann entweder ein chemischer Initiator wie AIBN verwendet werden oder die Reaktion startet durch Selbstinitiierung des Monomers. Chemischen Initiatoren, die auch photochemisch anregbar sind (z.B. AIBN), stören jedoch die zeitaufgelöste Messung. Daher erschien es sinnvoll, sogenannte dead-end Polymerisationen durchzuführen [3]. Dabei stoppt die Reaktion durch völliges Aufbrauchen des chemischen Initiators ohne Veränderungen der äußeren Bedingen bei einem durch die Anfangsinitiatorkonzentration vorgegebenen Umsatz. Für AIBN war allerdings die für einen Endumsatz von ca. $20 \%$ einzustellende Konzentration so gering, dass das AIBN die Reaktion nicht mehr steuern konnte. Vielmehr zeigte sich bei einer 
Reaktionstemperatur von $80{ }^{\circ} \mathrm{C}$ eine thermische Untergrundreaktion als bestimmend für den Verlauf der Reaktion, die sich auch nach Aufbrauchen des AIBN nicht merklich verlangsamte und bis zum vollständigen Umsatz verlief. Unter den oben beschriebenen Gesichtspunkten war also der Einsatz von AIBN für die Vorpolymerisation nicht von Vorteil.

Ebenfalls untersucht wurde tert-Amylperpivalat, das keine Absorption im Bereich der Laserwellenlänge zeigt und somit die zeitaufgelöste Messung nicht stören sollte. Trotzdem ist die Nutzung und die Handhabung von tert-Amylperpivalat nicht unproblematisch. Es kann auf Grund seiner hohen Zerfallskonstanten auch bei niedrigen Temperaturen zerfallen und so eine unerwünschte Nebenreaktion auslösen. Zusätzlich kann nicht ausgeschlossen werden, dass die Zerfallsprodukte des Peroxids die Polymerisation beeinflussen.

Wegen dieser Problematiken mit den chemischen Initiatoren wurden die Vorpolymerisationen schließlich meist durch rein thermische Reaktion des Monomers MMA durchgeführt. Als Reaktionsdruck wurden ein relativ niedriger Druck von 100 bar gewählt, da der Wachstumsgeschwindigkeitskoeffizient $k_{\mathrm{p}}$ bei diesem Druck verhältnismäßig klein ist [4] und somit kleinere, besser im Monomer lösliche Polymerketten produziert werden.

Als Reaktionstemperatur wurden $110^{\circ} \mathrm{C}$ eingestellt, um eine ausreichende Reaktionsgeschwindigkeit $\mathrm{zu}$ erreichen. Der bei den MMA Homopolymerisationen verwendete Photoinitiator DMPA ist bei dieser Temperatur stabil. Experimentell konnte keine Beschleunigung der thermischen Reaktion durch Zugabe unterschiedlicher DMPAKonzentrationen beobachtet werden.

Bei diesen Bedingungen wurden 20\% Monomerumsatz in ca. 1.5 Stunden erreicht. Danach beschleunigte sich die Reaktion durch den einsetzenden Geleffekt mit abnehmenden $k_{\mathrm{t}}$ stark. Während in einem ca. 5 min dauernden Zeitabschnitt in Bereichen unterhalb $20 \%$ Monomerumsatz nur ca. 1-2\% weiterer Umsatz erfolgte, ergab sich für das gleiche Zeitintervall bei $35 \%$ Monomerumsatz ein Fortschreiten der Reaktion um 5-7 \%. Diese starke Beschleunigung erschwerte mit zunehmender Reaktionszeit das kontrollierte Abrechen der Vorpolymerisation bei einem bestimmten Umsatz, zumal auch in der Abkühlphase weiterer Monomerumsatz erfolgte. Vorpolymerisationen ohne zusätzlichen Initiator bis $20 \%$ Monomerumsatz waren vergleichsweise gut reproduzierbar. Bei höheren angestrebten Umsätzen oder zusätzlichem chemischen Initiator war diese Reaktionskontrolle noch schwieriger. 


\subsection{Ablauf eines typischen zeitaufgelösten Experiments}

An dieser Stelle soll nur der grundsätzliche Messablauf dargestellt werden. Details zur Bedienung der Computerprogramme für die Steuerung der SP-PLP-Anlage finden sich im Anhang.

Zunächst wird die Hochdruckzelle, die nur eine leere Tefloninnenzelle enthält, bei der sich die Quarz-Fenster berühren, auf die Messtemperatur aufgeheizt und ein NIR-Eichspektrum aufgenommen. Anschließend wird die Zelle mit Heptan auf den gewünschten Druck gebracht. Der UV-Energiedetektor der SP-PLP-Anlage wird gegen ein Joulemeter (Gentec) geeicht.

Das gereinigte und entgaste Monomer wird in einem Handschuhkasten unter ArgonAtmosphäre mit dem vorher in einem $5 \mathrm{ml}$ Messkolben eingewogenen Initiator vermischt, bis dieser vollständig gelöst ist Zum Befüllen der Innenzelle wird ein Quarzfenster in den Teflonschlauch eingesetzt, die Messlösung eingefüllt und das zweite Quarzfenster mit Hilfe einer Pinzette eingesetzt. Die Innenzelle wird mit Hilfe des Teflonfüllkörpers in der Mitte der Hochdruckzelle fixiert, diese wird zugeschraubt und auf Reaktionsbedingungen gebracht.

Nach Aufnahme des FT-NIR-Startspektrums wird am Monochromator die genaue Wellenlänge des Absorptionsmaximums selektiert und die Grundhelligkeit des Analysenlichts gemessen.

Nach der Erfassung dieser Messgröße kann optional die in 5.4 beschriebene Vorpolymerisation durchgeführt werden.

Anschließend kann im Messprogramm, das u. a. die ADC (Analog Digital Converter) Karte des PCs steuert, die Anzahl der aufzusummierenden Einzelsignale vorgegeben werden. Das Messprogramm erfasst und kompensiert zusätzlich die Spannungen am InAs-Detektor vor und nach den Pulspaketen. Die weiteren Einstellungen, wie Zeitauflösung, etc. werden in dem Programm Sbench (v. 4.55, Fast ComTec) vorgenommen. Der Datenpunktabstand kann zwischen $2 \mu$ s und $1 \mathrm{~ms}$ variiert werden, die maximale Speichertiefe beträgt $2^{16}$ Datenpunkte. Das Datenerfassungsprogramm Sbench speichert die Änderung der Lichtintensität nach Auftreffen eines Laserpulses als zeitaufgelöstes Spannungssignal und mittelt gegebenenfalls über mehrere Signale. Die Daten werden zur weiteren Auswertung auf dem PC gespeichert. In Abständen von etwa $5 \%$ Monomerumsatz werden NIR-Spektren am FT-IR-Spektrometer aufgenommen, um den Gesamtmonomerumsatz zu bestimmen. 


\subsection{Ablauf der Vorpolymerisation}

Die vorbereitete Hochdruckzelle, in die eine Innenzelle eingesetzt wurde (siehe Abschnitt 4.1), wird mit Heptan als Druckmittel auf einen Druck von 100 bar gebracht. Es wird ein Referenzspektrum am FT-IR-Spektrometer aufgenommen und mittels eines Eurotherm-Thermoreglers auf $110{ }^{\circ} \mathrm{C}$ aufgeheizt. Dabei werden auch während der Aufheizphase in regelmäßigen Abständen IR-Spektren aufgenommen, um den Monomerumsatz zu kontrollieren. Ist ein bestimmter Umsatz erreicht (je nach Umsatzbereich 5 bis $10 \%$ vor dem eigentlich angestrebten Umsatz), wird die Zelle aus dem Spektrometer genommen und die Heizung ausgeschaltet. Die Hochdruckzelle wird mit Druckluft umspült, um eine möglichst schnelle Abkühlung zu gewährleisten. Die Abkühlphase dauert ca. 15 min. Hat sich die Hochdruckzelle annähernd bis zur gewünschten Temperatur abgekühlt, wird der Thermoregler wieder in Betrieb genommen und auf die Messtemperatur zurückgestellt. Während der Feineinstellung der Temperatur wird die Hochdruckzelle an den Druckstand angeschlossen und der Druck auf die Messbedingung eingeregelt. Nach einem weiteren Kontrollspektrum bei Messdruck kann das eigentliche SP-PLP-Experiment gestartet werden.

\subsection{Fehlerbetrachtung}

Die Temperatur wird in unmittelbarer Nähe der durchstrahlten Schicht mit einem ChromelAlumel-Thermoelement auf $\pm 0.5 \mathrm{~K}$ genau gemessen. Die Parameter des PID-Reglers wurden so optimiert, daß die Schwankungen bei $\pm 0.2 \mathrm{~K}$ liegen. Vom Hersteller wurde eine relative und eine absolute Genauigkeit von $\pm 0.5 \mathrm{~K}$ bzw. $\pm 1 \mathrm{~K}$ angegeben. Ein Temperaturgradient kann aufgrund der Konstruktion der Zelle, bei der auch der Luftraum vor den Fenstern erwärmt wird, weitgehend ausgeschlossen werden.

Der Startdruck in der Zelle wird mit einem geeichten Bourdon-Manometer, dessen Genauigkeit bei $\pm 0.1 \%$ des Skalenendwerts (3000 bar) liegt, bestimmt. Durch die bei der Polymerisation auftretende Volumenänderung ist der Druck in der Zelle nicht konstant. Eine Messung des Zellendrucks ist jedoch nachträglich nicht möglich, da die Hochdruckzelle während des Experiments nicht an das Manometer angeschlossen ist. Der Fehler wird auf $3 \%$ geschätzt.

Die Schichtlänge der Innenzelle wird mit einer Mikrometerschraube auf $0.05 \mathrm{~mm}$ genau bestimmt. Sie muss für die gewählten Messbedingungen über die Dichte der Monomermischung korrigiert werden, wodurch sich ein Fehler von $3 \%$ ergibt. 
Der Fehler der Initiator-Konzentrationen entsteht durch Fehler in der Einwaage und durch die Flüchtigkeit der Monomere. Der Fehler wird auf jeweils max. $2 \%$ geschätzt.

Die untere Grenze für quantitative Messungen mit dem FT-IR-Spektrometern liegt für die verwendeten Scanzahlen (100) bei Absorbanzen von 0.02. Für die zur Auswertung herangezogenen Absorbanzen zwischen 0.1 und 0.8 liegt der auftretende Fehler bei $1 \%$. Bei der Integration tritt durch ungenaue Bestimmung der Basislinie ein Fehler von weniger als $3 \%$ auf, der sich bei Halbbandenintegralen durch ungenaue Bestimmung des Maximums auf $5 \%$ erhöhen kann. Der für die kinetische Auswertung der Spektren entscheidende relative Fehler in den integralen Absorbanzen liegt bei unter $1 \%$.

Die zeitaufgelösten Messungen liefern die gekoppelten Parameter $k_{\mathrm{t}} / k_{\mathrm{p}}$ und $k_{\mathrm{t}} \cdot c_{\mathrm{R}}{ }^{0}$. Zur Bestimmung der Terminierungsgeschwindigkeitskoeffizienten ist somit die Kenntnis der Wachstumsgeschwindigkeitskoeffizienten notwendig, wenn $c_{\mathrm{R}}{ }^{0}$ unbekannt ist. Der Fehler für $k_{\mathrm{p}}$ aus der Literatur setzt sich fort, woraus ein Gesamtfehler für $k_{\mathrm{t}}$ von ca. $40 \%$ resultiert. Dieser Fehler ist jedoch als Durchschnittswert zu verstehen. So ist z. B. bei der MMA Homopolymerisation der Fehler in den zeitaufgelösten Messungen sehr unterschiedlich. Die Güte der Auswertung von einzelnen Messsignalen ist von verschiedenen Faktoren abhängig (s. Kapitel 6), der Fehler ist dabei als individuell anzusehen. Eine quantitative Angabe des Fehlers ist oft nicht möglich. Generell ist die Signalqualität bei höheren Umsätzen besser, gleichzeitig steigt jedoch die Unsicherheit für die Umsatzbestimmung. Die Reproduzierbarkeit innerhalb einer, bzw. analogen Messreihen ist von diesem Fehler zu unterscheiden, und wird in Kapitel 6 im Einzelnen diskutiert.

Für die Untersuchungen zur Kettenlängenabhängigkeit von $k_{\mathrm{t}}$ ergibt sich noch eine Problematik aus der Fitprozedur. Es hat sich gezeigt, dass die Parameter $k_{\mathrm{t}}^{0}$ und $\alpha$ in vielen Fällen miteinander korreliert sind. Somit lässt sich der Fehler des im wesentlichen betrachteten Parameters $\alpha$ nur schwer ermitteln. Er wird auf $50 \%$ geschätzt.

\subsection{Literatur Kapitel 5}

[1] C. Kowollik, Dissertation, Göttingen 1999.

[2] C. Schmaltz, Diplomarbeit, Göttingen 1994.

[3] H. G. Elias, Makromoleküle, Band 1, 5. Auflage, Hüthig \& Wepf Verlag, 1990.

[4] S. Beuermann, Dissertation, Göttingen 1993. 


\section{MMA-HOMOPOLYMERISATIONEN}

In diesem Kapitel werden die Untersuchungen zur Terminierungskinetik bei radikalischen MMA-Homopolymerisationen bis zu hohen Monomerumsätzen vorgestellt. Ziel war die Bestimmung von $k_{\mathrm{t}}$ und der Kettenlängenabhängigkeit dieses Koeffizienten mit der SP-PLPTechnik bei höheren Umsätzen. Entsprechend dem in Kapitel 3 vorgestellten Modell zur Umsatzabhängigkeit des Terminierungsgeschwindigkeitskoeffizienten soll $k_{\mathrm{t}}$ in diesem Bereich durch „Reaktionsdiffusionskontrolle“ bestimmt werden. Die Untersuchungen waren von erheblichen experimentellen Schwierigkeiten begleitet, auf die zu Beginn dieses Kapitels eingegangen wird. Die $k_{\mathrm{t}}$-Werte der einzelnen Messungen wurden in Zusammenarbeit mit Thomas Junkers erhalten und sind bei Junkers [1] in Tabellenform abgedruckt.

\subsection{Vorbemerkungen zur Auswertung}

Dieser Abschnitt soll einige bei den Untersuchungen aufgetretene Probleme und deren Ursachen vorstellen. Dazu zählen das bei diesen Experimenten meist schlechte SignalRausch-Verhältnis sowie die bei hohen Umsätzen beobachteten Inhomogenitäten der Reaktionsmischung.

\subsubsection{Signal-Rausch-Verhältnis}

Ursächlich für ein Rauschen im Messsignal können Schwankungen in der Lichtintensität der IR-Lampe, im Detektorsignal oder in der Messelektronik sein. Zusätzlich können trotz der gedämpften Lagerung des optischen Aufbaus nicht völlig zu vermeidende Erschütterungen der Apparatur im Signal sichtbar werden. Das Signal-Rausch-Verhältnis wird durch steigenden Umsatz pro Laserpuls verbessert. Dazu muss das Verhältnis vom Wachstumsgeschwindigkeitskoeffizient $k_{\mathrm{p}}$ zum Terminierungsgeschwindigkeitskoeffizient $k_{\mathrm{t}}$ möglichst groß sein. In Abbildung 6.1 sind $k_{\mathrm{p}}$ und (kettenlängengemittelte) $k_{\mathrm{t}}$ für einige Acrylate und Methacrylate bei $40{ }^{\circ} \mathrm{C} / 1000$ bar dargestellt [2]. Deutlich sichtbar ist die Gruppierung der Acrylate einerseits und der Methacrylate andererseits $\mathrm{zu}$ Familien bezüglich des Wachstumsgeschwindigkeitskoeffizienten $k_{\mathrm{p}}$, wohingegen $k_{\mathrm{t}}$ wesentlich durch die Größe des Esterrestes bestimmt wird. Bei der Diskussion der Kettenlängenabhängigkeit von $k_{\mathrm{t}}$ in Kapitel 8 wird detailliert auf den Einfluss des Esterrestes auf $k_{\mathrm{t}}$ eingegangen. Dort zeigt sich, dass die hier gewählte Darstellungsweise bei Acrylaten weiterer Erläuterungen bedarf. 
Durch das vergleichsweise hohe $k_{\mathrm{t}}$ und kleine $k_{\mathrm{p}}$ ist MMA von den in Abbildung 6.1 gezeigten Monomeren das für die SP-PLP-Technik am wenigsten geeignete. Bei niedrigen Monomerumsätzen lässt sich nur durch Coaddition vieler Einzelsignale eine ausreichende Signalqualität erreichen.

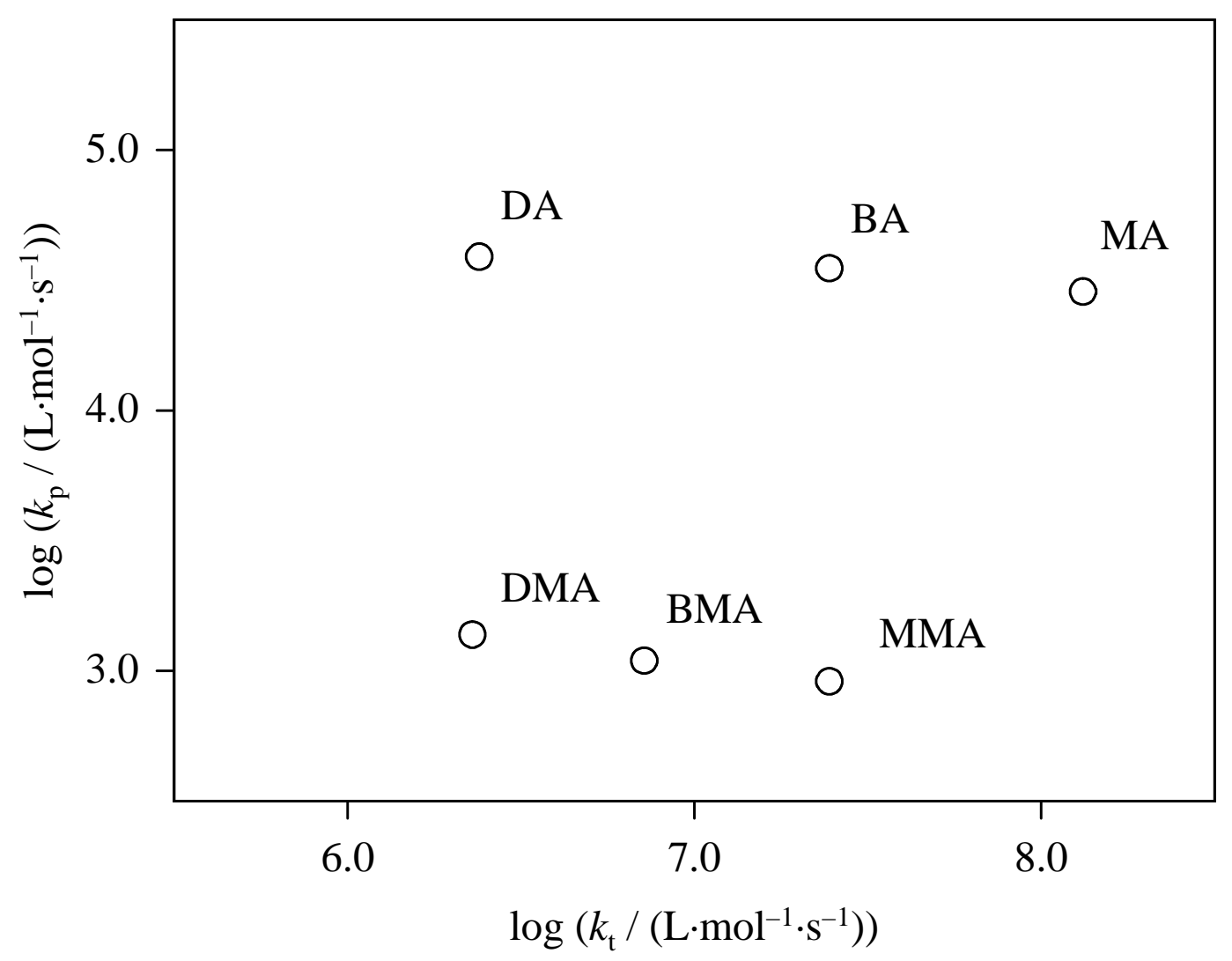

Abb. 6.1: Wachstumsgeschwindigkeitskoeffizienten $k_{\mathrm{p}}$ und Terminierungsgeschwindigkeitskoeffizienten $k_{\mathrm{t}}$ einiger Acrylate und Methacrylate bei $40^{\circ} \mathrm{C} / 1000$ bar und niedrigen Umsätzen.

Bei höheren Monomerumsätzen fällt $k_{\mathrm{t}}$ von MMA gemäß den Erläuterungen in Kapitel 3 stark ab und das Problem eines schlechten Signal-Rausch-Verhältnisses verringert sich merklich. Der Umstand, dass bei höheren Monomerumsätzen auch weniger Monomer zur Reaktion bereit steht, wird durch den Abfall von $k_{\mathrm{t}}$ deutlich überkompensiert. So müssen etwa bei $40{ }^{\circ} \mathrm{C}$ / 1000 bar und niedrigen Umsätzen bis zu 100 Einzelsignale für ein auswertbares Signal gemittelt werden, während bei hohen Umsätzen ein einzelnes Signal ausreicht. 


\subsubsection{Homogenität der Reaktionsmischung}

Bei der Bestimmung von $k_{\mathrm{t}}$ für MMA bei höheren Monomerumsätzen ergab sich als wesentliches Problem die aufgetretene Inhomogenität der Monomer-Polymer-Mischung. Messungen mit der SP-PLP-Technik sind sehr empfindlich und lassen sich an inhomogen Reaktionsmischungen nicht durchführen. Solche Mischungen werden vom Analysenlicht nicht vollständig durchstrahlt und erlauben keine korrekte zeitaufgelöste Aufnahme der Änderung der Monomerkonzentration. Zudem können die bei Inhomogenitäten bedeutsamen Mischungseffekte in der Auswertung nicht berücksichtigt werden.

Die Homogenität der Monomer-Polymer-Mischung wird durch zahlreiche Faktoren beeinflusst. Die Lösungsmitteleigenschaften des Monomers verändern sich mit Druck und Temperatur. Von großer Wichtigkeit sind außerdem Monomerumsatz sowie Größe und Struktur der Polymerketten. Die Polymereigenschaften werden wiederum durch die Reaktionsbedingungen wie Druck und Temperatur, die Art der Initiierung sowie die InitiatorKonzentration bestimmt.

Hohe Initiatorkonzentrationen führen wegen der größeren Terminierungswahrscheinlichkeit für die einzelne Kette zu kleineren Polymermolmassen und damit einer besseren Löslichkeit im Monomer.

Bei einer Erhöhung der Temperatur oder des Drucks werden durch das größere $k_{\mathrm{p}}$ längere Polymerketten gebildet und so verschlechtert sich die Homogenität der Mischung. Andererseits wird dies möglicherweise durch eine Steigerung der Löslichkeit bei diesen Bedingungen kompensiert. Eine Abschätzung, welcher Effekt überwiegt, ist schwierig, jedoch sind wegen des oben beschriebenen Signal-Rausch-Verhältnisses hohe Drücke für SP-PLPExperimente vorzuziehen.

Schließlich ist die Art der Initiierung der Polymerisation ebenfalls von entscheidender Bedeutung für die Homogenität der Mischung. In den vorangehenden Kapiteln wurde bereits erläutert, dass die Initiierung auf thermischem, chemischem oder photochemischem Wege möglich ist. Die beiden erstgenannten Alternativen ergeben eine ständige Produktion von Radikalen unter stationären Bedingungen. Dadurch stehen jederzeit kleine, bewegliche Radikalketten zur Verfügung und es kann von einer insgesamt größeren Terminierungswahrscheinlichkeit für die einzelne Kette auch bei höheren Monomerumsätzen ausgegangen werden. Das führt zu einer geringeren mittleren Molmasse und damit zu besserer Löslichkeit des Polymers im Monomer. 
Bei der in dieser Arbeit im Wesentlichen verwendeten photochemischen Initiierung wird in sehr kurzer Zeit eine Radikalpopulation erzeugt, die dann gleichzeitig abreagiert. Durch das Fehlen neuer mobiler Radikale sollte es besonders bei höheren Umsätzen mit eingeschränkter Translation der langen Ketten zu weniger Terminierung kommen und so Polymer mit großer Molmasse und schlechter Solvatation erzeugt werden.

Experimentell konnte der vermutete Unterschied in den Molmassenverteilungen allerdings aufgrund des Erreichens der Ausschlussgrenze der zu Verfügung stehenden GPC-Apparatur nicht nachgewiesen werden. Des Weiteren ist die Reaktionsgeschwindigkeit bei der thermischen oder chemischen Radikalproduktion niedriger und so eine homogene Durchmischung innerhalb der Zelle wahrscheinlicher.

Außerdem bestehen zwischen den beschriebenen Initiierungstechniken Unterschiede hinsichtlich der Verteilung der neu gebildeten Radikale in der Mischung. Bei der thermischen oder chemischen Initiierung kann von einer gleichmäßigen Radikalverteilung ausgegangen werden. Bei der photochemischen Initiierung ist es denkbar, dass eine einheitliche Bestrahlung des gesamten Probevolumens nicht vollständig gegeben ist. Mit Hilfe des Lambert-Beerschen Gesetzes und der verwendeten Schichtlänge von etwa $1.4 \mathrm{~mm}$ lässt sich abschätzen, dass die Intensität des Laserlichts in Durchstrahlrichtung nur wenig abnimmt und entlang dieser Achse kein Konzentrationsgradient der Radikale vorliegt. Eine unterschiedliche Energieverteilung über die bestrahlte Fläche der Zelle ist jedoch denkbar. Dadurch kommt es in der Innenzelle zu einer Ungleichverteilung der Radikale, die sich bei hohen Umsätzen nicht mehr ausreichend schnell ausgleichen kann. Eine Messung der Energieverteilung war nicht möglich, da kein entsprechender Detektor zur Verfügung stand. Es wurde jedoch schon früher beobachtet, dass durch Einstrahlen von Laserpulsen bei höheren Umsätzen sichtbare Konzentrationsunterschiede in der Zelle entstehen können [3].

Die photochemische Anregung mittels eines Lasers führt also im Vergleich zu thermischer oder chemischer Initiierung bevorzugt zu Inhomogenitäten in der Reaktionsmischung.

\subsection{Erweiterung des zugänglichen Umsatzbereiches}

MMA gehört zu der in Kapitel 3 beschriebenen Klasse der Typ B Monomere mit einer komplizierten Umsatzabhängigkeit des Terminierungsgeschwindigkeitskoeffizienten [4]. Mit der SP-PLP-Technik konnten bei MMA bislang nur niedrige Monomerumsätze erreicht werden. Der Grund dafür liegt im bereits in Abschnitt 6.1.1 erwähnten ungünstigen SignalRausch-Verhältnis bei MMA-Homopolymerisationen. Insbesondere im Segmentdiffusions- 
bereich ist die Coaddition vieler Einzelsignale bei den SP-PLP-Messungen nötig. Folglich ist der Verbrauch an Photoinitiator so hoch, dass bereits bei Umsätzen um $20 \%$ keine ausreichende Menge an Initiator mehr vorliegt. Eine Erhöhung der InitiatorAnfangskonzentration (hier $5 \cdot 10^{-3} \mathrm{~mol} \cdot \mathrm{L}^{-1}$ ) als Ausgleich ist im Fall von DMPA nicht wirkungsvoll, da mehr Initiator hier sogar für weniger Umsatz pro Laserpuls sorgt [5] (siehe Abschnitt 6.5). Den in diesem Abschnitt beschriebenen photochemisch initiierten Reaktionen wurde deshalb die in Kapitel 5 erläuterte Vorpolymerisation vorangestellt, um die gewünschten mittleren und höheren Umsätze erreichen zu können.

Für die Terminierungsreaktion ist die Viskosität der Reaktionsmischung und damit die Molmasse der Polymermoleküle von großer Wichtigkeit. Deshalb sollte zunächst untersucht werden, ob das bei der Vorpolymerisation hergestellte Polymer eine ähnliche Molmasse wie das bei der photoinduzierten Polymerisation gebildete Polymer aufweist.

Diese Bedingung musste gewährleistet sein für eine Vergleichbarkeit von SP-PLPMessungen, denen eine Vorpolymerisation vorausgeht, mit alten Ergebnissen für den Segmentdiffusionsbereich ohne Vorpolymerisation [2,6]. Die eigentlichen SP-PLPMessungen wurden ebenfalls aus diesem Grund analog zu Kowollik [2,6] bei $40{ }^{\circ} \mathrm{C} /$ 1000 bar durchgeführt.

Abbildung 6.2 zeigt die GPC-Analyse einer Probe aus rein thermischer Vorpolymerisation zusammen mit Poly-MMA hergestellt mit Vorpolymerisation und anschließender photochemischer Polymerisation.

Die beiden Molmassenverteilungen in Abbildung 6.2 stimmen gut überein. Bei der verwendeten GPC-Anlage liegt die Ausschlussgrenze, ab der Molmassen nicht mehr unterschieden werden können, bei etwa $3 \cdot 10^{6} \mathrm{~g} \cdot \mathrm{mol}^{-1}$. In den gezeigten Kurven ist bei Molmassen, die über der Ausschlussgrenze liegen, ein steiler Abfall $\mathrm{zu}$ erkennen. Das deutet auf eine unzureichende Trennung in diesem Bereich hin. 


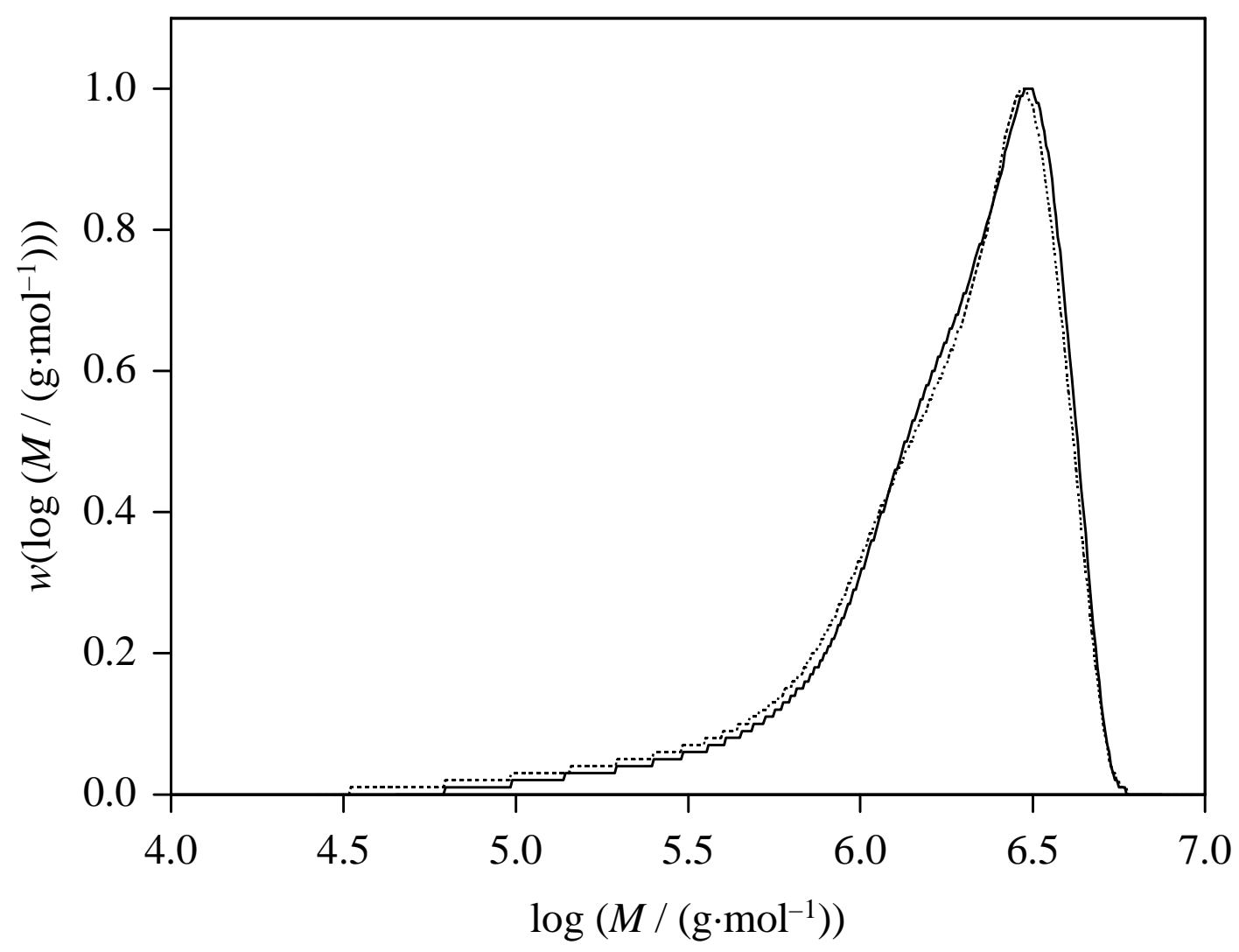

Abb. 6.2: Normierte Molmassenverteilungen von MMA-Proben aus thermischer Vorpolymerisation bei $110^{\circ} \mathrm{C} / 100$ bar (durchgezogene Linie) sowie aus Vorpolymerisation $\left(110^{\circ} \mathrm{C} / 100\right.$ bar $)$ mit anschließender photochemischer Polymerisation bei $40{ }^{\circ} \mathrm{C} / 1000$ bar.

Da also bei Molmassen größer $3 \cdot 10^{6} \mathrm{~g} \cdot \mathrm{mol}^{-1}$ keine Unterscheidung mit der zur Verfügung stehenden GPC möglich war, kann nicht entschieden werden, ob die Polymerproben auch bei sehr hohen Molmassen übereinstimmen. Im Rahmen der Messungenauigkeit kann aber davon ausgegangen werden, dass die Vorpolymerisation ein Polymer erzeugt, das dem photochemisch produzierten Polymer hinreichend ähnelt.

Somit wurden SP-PLP-Experimente mit Vorpolymerisationen bis 15, 20 und $35 \%$ Monomerumsatz zur Erweiterung des für Einzelpuls-Messungen zugänglichen Umsatzbereichs durchgeführt. Die erhaltenen Ergebnisse für $40^{\circ} \mathrm{C} / 1000 \mathrm{bar}$ sind in Abbildung 6.3 illustriert. 


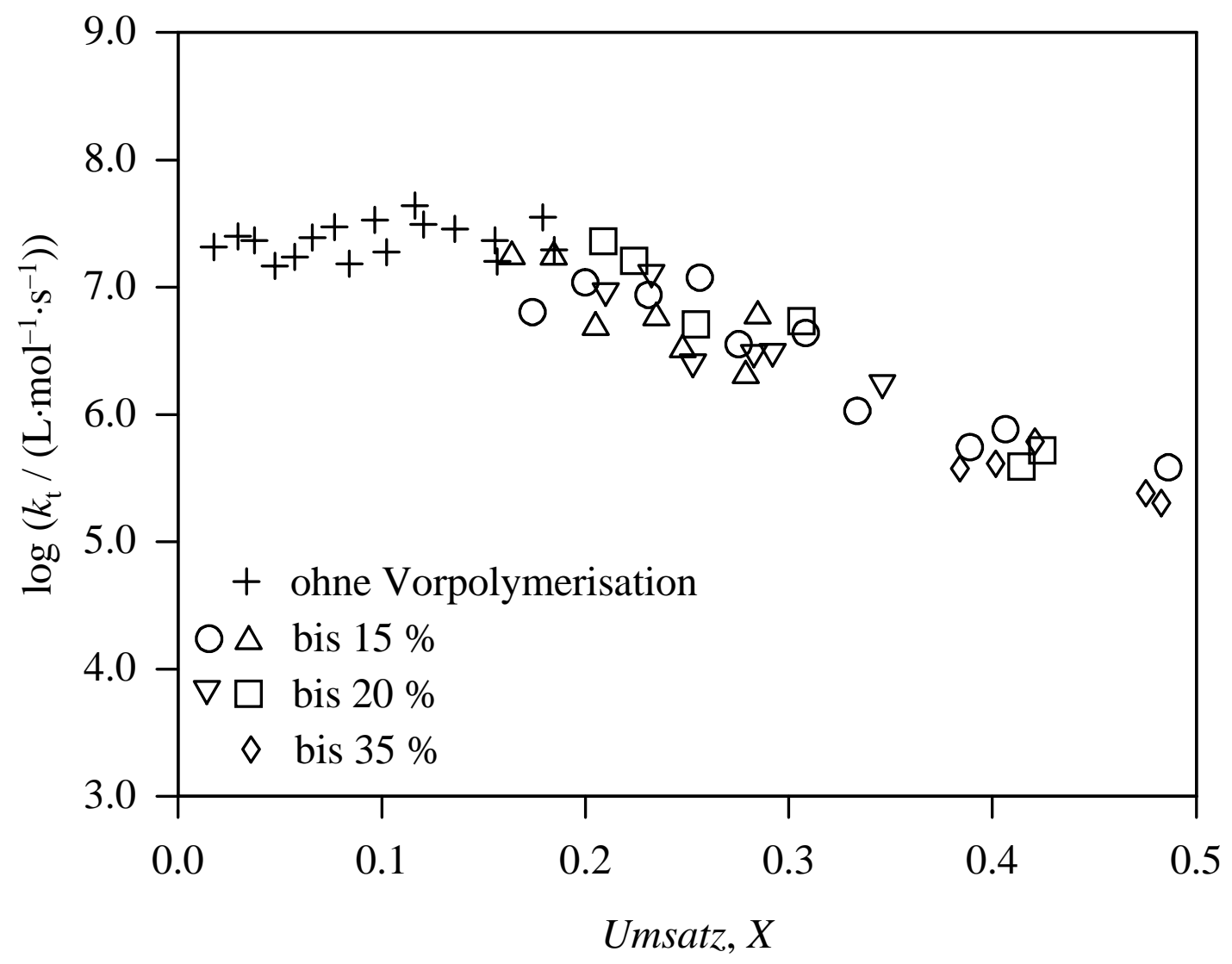

Abb. 6.3: SP-PLP-Messungen von MMA-Homopolymerisationen bei $40{ }^{\circ} \mathrm{C} / 1000$ bar. Die unterschiedlichen Symbole kennzeichnen unabhängige Messreihen. Die mit + gekennzeichneten Daten stammen von Kowollik [2, 6].

Die gezeigten Daten stimmen innerhalb der Messungenauigkeit überein. Die $k_{\mathrm{t}}$-Werte aus verschiedenen Messreihen, bei denen unterschiedlich lange vorpolymerisiert wurde, schließen aneinander an. Dort, wo sich die Umsatzbereiche überlappen, geling eine Reproduktion der Daten. Die mit der Vorpolymerisation erhaltenen Ergebnisse passen außerdem gut zu den als Kreuze dargestellten Werten für SP-PLP-Experimente bei Umsätzen kleiner $20 \%$ ohne Vorpolymerisation von Kowollik [2,6]. Die Streuung von bis zu einer dekadischen logarithmischen Einheit ist bei den neuen Ergebnissen allerdings etwas größer als bei Kowollik. Ein Grund dafür liegt in der Vorpolymerisation als zusätzlicher Fehlerquelle, bei der sich kleine Abweichungen in den folgenden Werten deutlich bemerkbar machen können. Der in Kapitel 3 beschriebene theoretische Kurvenverlauf von $k_{\mathrm{t}}$ ist in der Abbildung 6.3 erkennbar. Mit Einsetzen des Geleffekts bei ca. $20 \%$ Monomerumsatz fällt $k_{\mathrm{t}}$ stetig bis fast $50 \%$ Umsatz. Der Bereich der Reaktionsdiffusionskontrolle mit nur noch geringem Abfall des Terminierungsgeschwindigkeitskoeffizienten wurde in diesen Messungen wegen der 
vorher aufgetretenen Inhomogenität der Reaktionsmischung nicht erreicht. Wie schon in Abschnitt 6.1.2 beschrieben, werden die Reaktionsmischungen bei laserinduzierten Polymerisationen schneller heterogen.

Zur Erweiterung des zugänglichen Umsatzbereiches erschien der zusätzliche Einsatz eines thermisch zerfallenden Initiators während der Vorpolymerisation sinnvoll. Dadurch sollte es zu einer Bildung von Polymer mit geringerer Molmasse und besserer Homogenität der Reaktionsmischung kommen. Als Initiatoren wurden in dieser Arbeit AIBN und tert-Amylperoxypivalat verwendet. Bei AIBN tritt das Problem auf, dass es auch photochemisch anregbar ist und während des SP-PLP-Experiments zerfallen würde. Es ergäbe sich also eine Überlagerung mit der eigentlichen Initiierung durch DMPA und durch den andersartigen Zerfallsmechanismus eine deutliche Erschwernis der Auswertung. AIBN sollte deshalb am Ende der Vorpolymerisation vollständig verbraucht sein. Dazu müsste die Anfangskonzentration jedoch so gering gewählt werden, dass kein nennenswerter Einfluss auf das Reaktionsgeschehen stattfinden würde. Bei tert-Amylperoxypivalat gibt es wegen der nicht vorhandenen Absorbanz im UV-Bereich kein Problem mit Nebenreaktionen während der SP-PLP-Experimente. Außerdem ist die Zerfallsgeschwindigkeit bei der Temperatur der Vorpolymerisation $\left(\tau_{1 / 2}=90 \mathrm{~s}\right.$ bei $110^{\circ} \mathrm{C}$ [7]) so groß, dass man von einem fast vollständigen Verbrauch vor Beginn der laserinduzierten Messungen ausgehen kann. Das Pivalat ist aber schon bei Zimmertemperatur recht instabil und somit ist eine Vorpolymerisation mit diesem Initiator schwierig und auch für einen definierten Monomerumsatz nicht gut reproduzierbar. Diese Problematik ergibt sich für alle bei relativ niedriger Temperatur zerfallenden Peroxide. Der Einsatz eines chemischen Initiators während der Vorpolymerisation erwies sich also bei den im Rahmen dieser Arbeit durchgeführten Untersuchungen als nicht vorteilhaft. Eine reproduzierbare Erweiterung des Umsatzbereiches im Vergleich zu Messungen mit rein thermischer Vorpolymerisation wurde so nicht erreicht. In diesem Kapitel werden deshalb nur Ergebnisse dargestellt, bei denen die Vorpolymerisation ohne chemischen Initiator vorgenommen wurde.

\subsection{Untersuchungen im Bereich der „,Reaktionsdiffusionskontrolle“}

Zur Bestimmung der Reaktionsdiffusionskonstante $C_{\mathrm{RD}}$ sind Messungen bei höheren Umsätzen notwendig. Im Rahmen dieser Arbeit liegen jedoch aufgrund von oftmals vorher eingesetzter Inhomogenität nur wenige Daten für den Bereich vor, in dem $k_{\mathrm{t}}$ entsprechend dem in Kapitel 3 vorgestellten Modell „reaktionsdiffusionskontrolliert“ ist. Erfolgreiche 
Messungen im gewünschten Umsatzbereich gelangen wegen der besseren Homogenität der Reaktionsmischung nur bei einem auf 2000 bar erhöhten Druck. Der dann zu beobachtende flachere Verlauf im Vergleich zur Region des Geleffekts wurde bei den Experimenten bei ca. $50 \%$ Monomerumsatz erreicht. Die entsprechenden Daten für höhere Umsätze sind in der Abbildung 6.4 gezeigt.

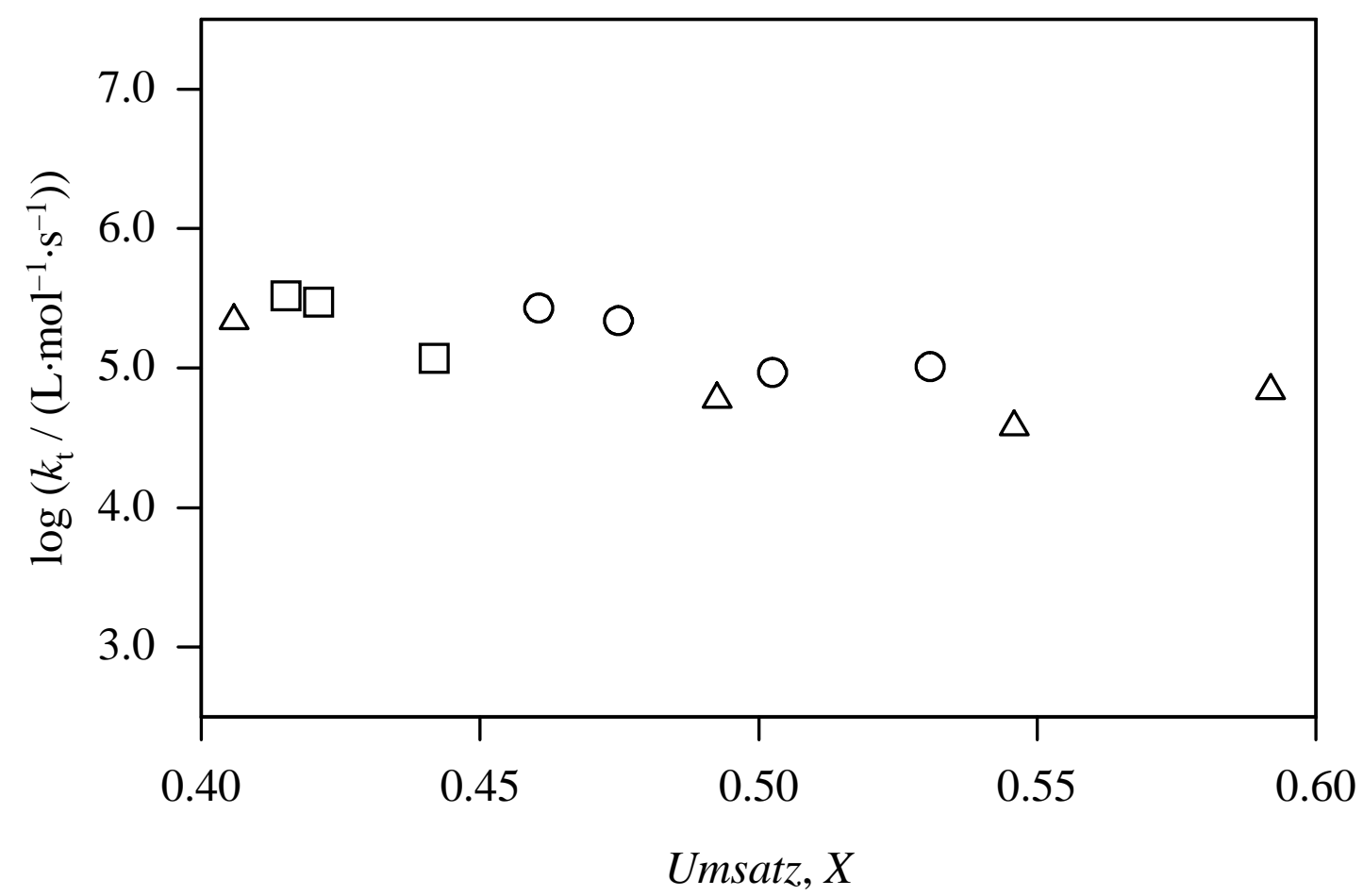

Abb. 6.4: $\log \left(k_{\mathrm{t}} /\left(\mathrm{L} \cdot \mathrm{mol}^{-1} \cdot \mathrm{s}^{-1}\right)\right)$ als Funktion des Monomerumsatzes für $40{ }^{\circ} \mathrm{C} / 2000$ bar mit $c(\mathrm{DMPA})=5 \cdot 10^{-3} \mathrm{~mol} \cdot \mathrm{L}^{-1}$. Verschiedene Symbole kennzeichnen unabhängige Messungen.

Für die in Abbildung 6.4 gezeigten Messungen ergibt sich für den Bereich der Reaktionsdiffusionskontrolle ein Absolutwert von $\log \left(k_{\mathrm{t}} /\left(\mathrm{L} \cdot \mathrm{mol}^{-1} \cdot \mathrm{s}^{-1}\right)\right)=4.9 \pm 0.2$.

\section{Bestimmung der Reaktionsdiffusionskonstanten $C_{\mathrm{RD}}$}

Mit den primär aus der SP-PLP-Messung erhaltenen gekoppelten Parametern $k_{\mathrm{t}} / k_{\mathrm{p}}$ kann $C_{\mathrm{RD}}$ bei Kenntnis des Monomerumsatzes mittels Gleichung 3.12 für jeden Messpunkt direkt berechnet und gegebenenfalls über mehrere Punkte gemittelt werden. In der Abbildung 6.5 ist $k_{\mathrm{t}} /\left(k_{\mathrm{p}} \cdot(1-X)\right)$ bzw. $C_{\mathrm{RD}}$ für die Experimente bei 2000 bar (aus Abbildung 6.4) dargestellt. Aus den vorhandenen Daten kann die Reaktionsdiffusionskonstante $C_{\mathrm{RD}}$ berechnet werden. Sie ist zusammen mit einigen Literaturdaten in Tabelle 6.1 aufgeführt. 


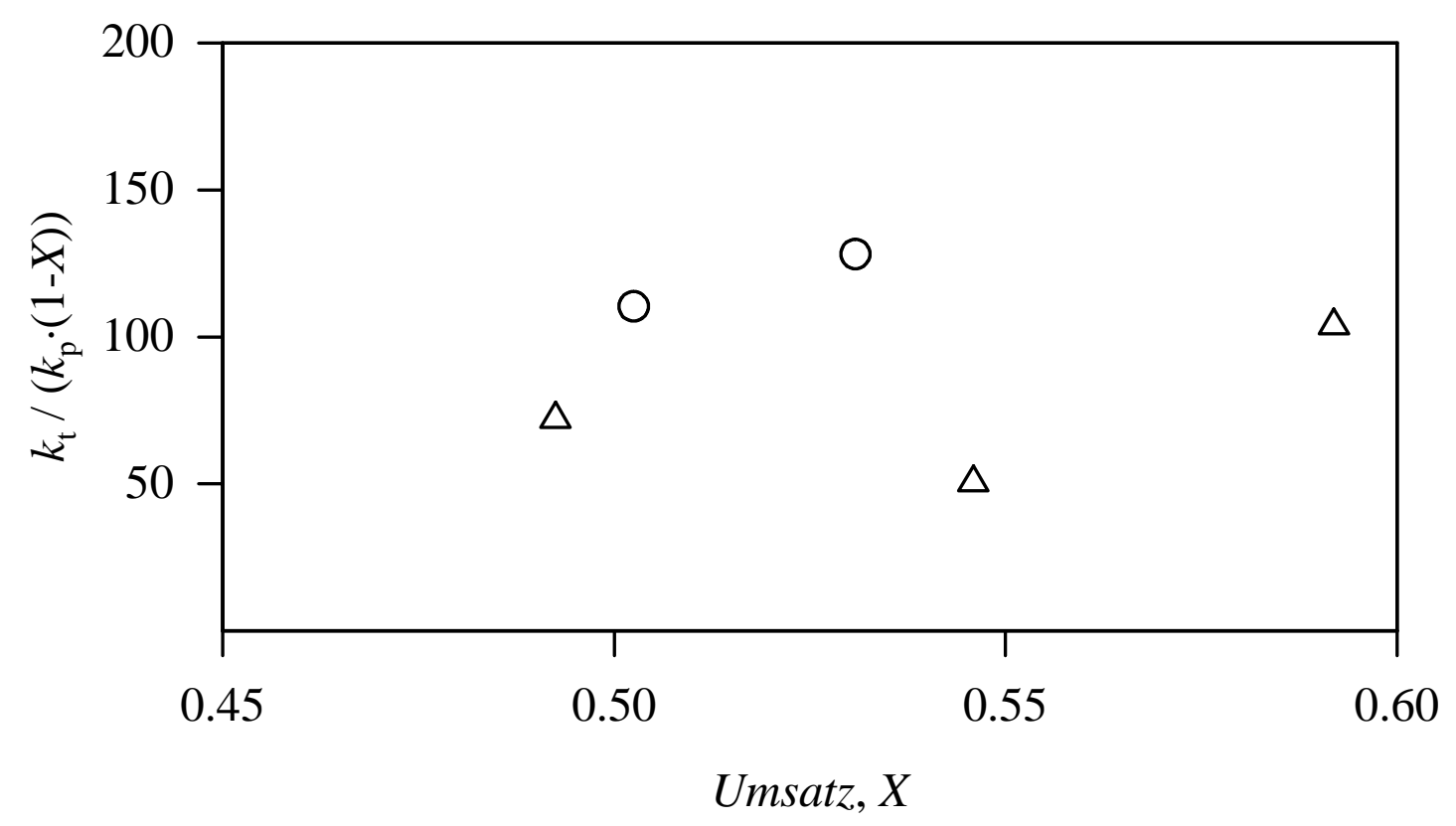

Abb. 6.5: $C_{\mathrm{RD}}$ als Funktion des Umsatzes bei $40{ }^{\circ} \mathrm{C} / 2000$ bar. Unterschiedliche Symbole kennzeichnen unabhängige Messreihen.

\begin{tabular}{c|c|ccc}
\hline & (a) & (b) & (c) & (d) \\
\hline$p /$ bar & 2000 & 1000 & 1 & 1 \\
$T /{ }^{\circ} \mathrm{C}$ & 40 & 30 & 50 & 0 \\
$C_{\mathrm{RD}}$ & $95 \pm 40$ & 76 & $84 \pm 25$ & 94 \\
\hline
\end{tabular}

Tab. 6.1: Die Reaktionsdiffusionskonstante $C_{\mathrm{RD}}$ bei verschiedenen Drücken, Temperaturen und Messmethoden; (a) diese Arbeit, (b) nach Beuermann [8], (c, d) nach Buback et al. [4].

Im Rahmen der Messungenauigkeit stimmt der in dieser Arbeit bestimmte Wert für $C_{\mathrm{RD}}$ mit den Daten aus der Literatur überein. Über eine von Buback et al. diskutierte Druckabhängigkeit für die Reaktionsdiffusionskonstante [4, 9] kann mit den in Tabelle 6.1 dargestellten Daten nicht entschieden werden. Buback geht von einer Abnahme von $C_{\mathrm{RD}}$ mit steigendem Druck aus. Es wurde ein Aktivierungsvolumen von $(16 \pm 6) \mathrm{cm}^{3} \cdot \mathrm{mol}^{-1}$ für $C_{\mathrm{RD}}$ gefunden [9], jedoch für das Klasse A Monomer BA. Von einer Temperaturabhängigkeit ist nichts bekannt. Innerhalb der Literaturwerte (b-d) könnte man die von Buback erwartete Tendenz erkennen. Der Literaturwert aus (c) bei $50{ }^{\circ} \mathrm{C} / 1$ bar schließt jedoch innerhalb der 
angegebenen Fehlergrenzen die anderen Literaturwerte aus (b) und (d) mit ein. Der in dieser Arbeit bestimmte Wert für $C_{\mathrm{RD}}$ sollte bei einer Druckabhängigkeit der niedrigste in der Tabelle sein, was nicht der Fall ist. Mit dem in der Literatur angegeben Aktivierungsvolumen [9] würde sich für bei einer Umrechnung des in dieser Arbeit erhaltenen Ergebnisses auf 1000 bar ein Wert von $C_{\mathrm{RD}}=175$ ergeben, was auch unter Beachtung des Fehlers eine deutliche Abweichung vom Literaturwert von Beuermann [8] darstellt. Allerdings gibt der aufgeführte Fehler nur die statistische Abweichung an. Die eigentliche Ungenauigkeit aufgrund der oben beschriebenen Messungenauigkeit kann als noch größer angenommen werden.

\subsection{Untersuchungen mit dem Transferagens DDM}

Wie bereits mehrfach erwähnt, führten Inhomogenitäten der Reaktionsmischung in einigen Fällen zum Abbruch von SP-PLP-Experimenten vor Erreichen des Umsatzbereiches, in dem $k_{\mathrm{t}}$ gemäß dem in Kapitel 3 vorgestellten Modell der „Reaktionsdiffusionskontrolle“ unterliegt. Durch den Einsatz von DDM als Kettenübertragungsagens sollte der in homogener Phase zugängliche Monomerumsatz erhöht werden.

Die Wirkungsweise von Transferagenzien wurde bereits in Kapitel 3.3 erläutert. Diese Substanzen greifen in die Polymerisationsreaktion derart ein, dass sie die Radikalfunktion einer wachsenden Kette übernehmen und so totes Polymer erzeugen. Das Transferagens kann nun seinerseits mit einem Monomermolekül reagieren und eine neue Kette starten. In Abhängigkeit von der Reaktivität und Konzentration des Kettenüberträgers lässt sich die Anzahl dieser Reaktionen und damit die Molmasse der Polymermoleküle steuern. Es gibt katalytisch wirkende Agenzien, aber auch solche, die in die Polymerkette eingebaut und so während der Reaktion verbraucht werden [10, 11, 12]. Das in dieser Arbeit verwendete DDM zählt zum zweiten Typ von Kettenüberträgern.

In diesem Kapitel wird für die Auswertung der SP-PLP-Signale von idealer Polymerisationskinetik ausgegangen. Mit dem Programmpaket Predici ${ }^{\circledR}$ kann jedoch gezeigt werden, dass trotz des Hinzufügens eines zusätzlichen Reaktionsschrittes durch das DDM der Umsatz-Zeit-Verlauf bei den laserinduzierten Messungen unverändert bleibt. Dies ist in Abbildung 6.6 dargestellt. 

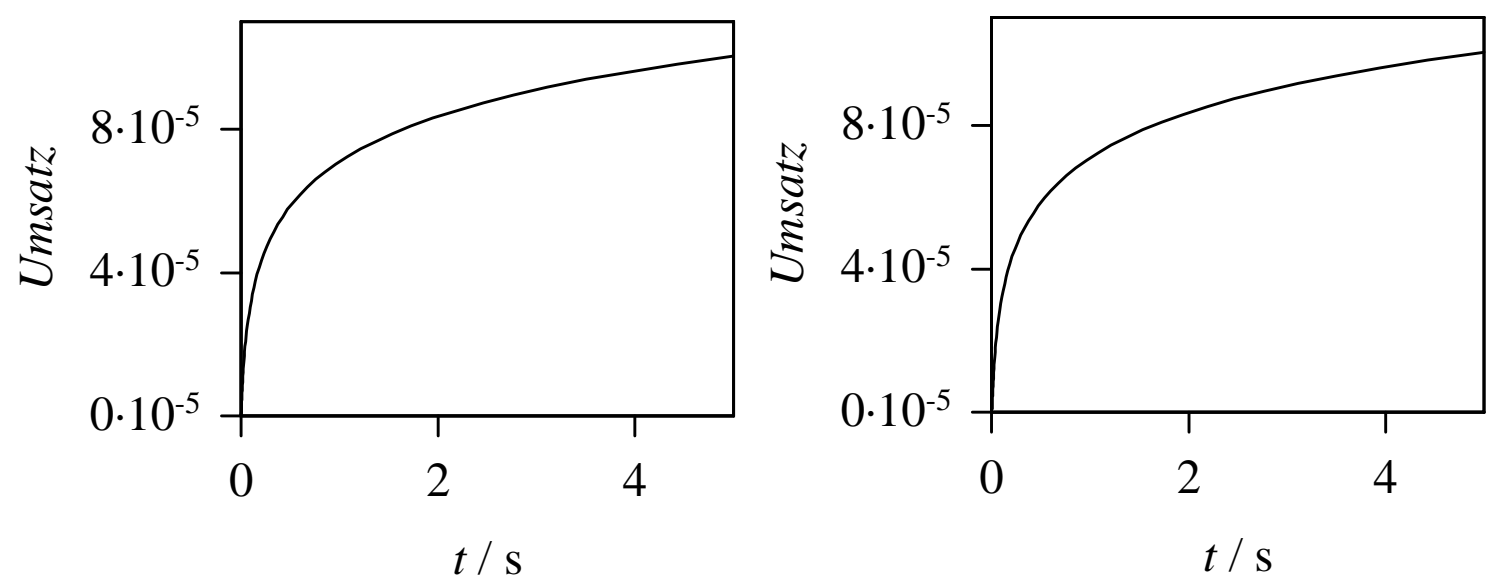

Abb. 6.6: Mit dem Programmpaket Predici ${ }^{\circledR}$ simulierte Umsatz-Zeit-Verläufe ohne (links) sowie mit Zusatz eines Kettenüberträgers (rechts) bei ansonsten gleichen Reaktionsbedingungen.

Durch die Zugabe von DDM ändert sich an der Fitprozedur nach Gleichung 3.6 bei angenommener idealer Kinetik nichts, da im Transferschritt kein Monomer verbraucht wird und die Reaktion schnell ist. Der Geschwindigkeitskoeffizient für die Transferreaktion $k_{\text {tr }}$ ist unter den verwendeten Messbedingungen mit $500 \mathrm{~L} \cdot \mathrm{mol}^{-1} \cdot \mathrm{s}^{-1}$ etwa halb so groß wie $k_{\mathrm{p}}$ [13].

\section{SP-PLP-Messungen mit DDM}

Die Experimente wurden analog zu den SP-PLP-Messungen ohne DDM bei $40{ }^{\circ} \mathrm{C} / 1000$ bar durchgeführt. Im Vergleich $\mathrm{zu}$ den oben beschriebenen Untersuchungen war bei den Experimenten mit DDM auch im mittleren Umsatzbereich das Coaddieren von vielen Laserpulsen pro Signal notwendig. Das Signal-Rausch-Verhältnis war also dort, wo es sich eigentlich bei Auftreten eines Geleffekts bessern müsste, ähnlich schlecht wie zu Beginn der Polymerisation. Allein dieser Befund lässt auf ein vergleichsweise großes $k_{\mathrm{t}}$ bis zu höheren Monomerumsätzen schließen. Das Coaddieren von vielen Laserpulsen führte dazu, dass pro Messreihe nicht mehr als $10 \%$ Monomerumsatz untersucht werden konnten. Bei den zugehörigen Vorpolymerisationen war es somit notwendig, von den oben diskutierten Untersuchungen abweichende Startumsätze für die SP-PLP-Messungen einzustellen.

Die Experimente mit Zusatz von DDM gelangen mit einer relativ geringen Streuung von 0.5 logarithmischen Einheiten. Sie sind in Abbildung 6.7 zusammen mit ähnlichen Messungen ohne Zugabe von DDM (aus Abschnitt 6.2) dargestellt. 


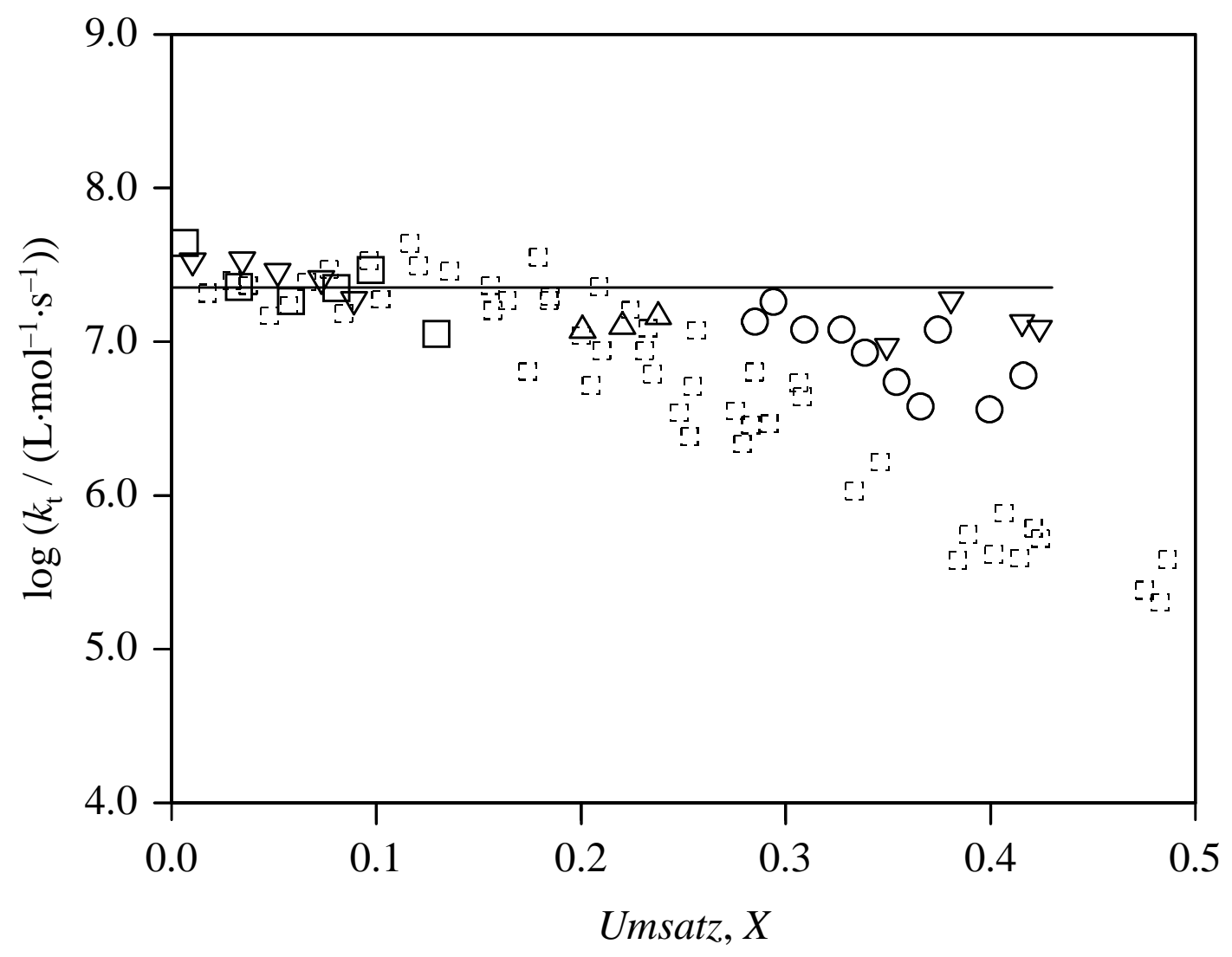

Abb. 6.7: $\log \left(k_{\mathrm{t}} /\left(\mathrm{L} \cdot \mathrm{mol}^{-1} \cdot \mathrm{s}^{-1}\right)\right)$ als Funktion des Umsatzes für Experimente mit Zusatz von DDM bei $40{ }^{\circ} \mathrm{C} / 1000$ bar. Unterschiedliche Symbole zeigen unabhängige Messreihen. Als gestrichelte Symbole sind entsprechende Messungen ohne DDM aus Abbildung 6.3 eingezeichnet.

Bei den Untersuchungen mit DDM ändert sich, wie bereits vermutet, $k_{\mathrm{t}}$ im gesamten gemessenen Umsatzbereich nur wenig. Es ist im Unterschied $\mathrm{zu}$ den Experimenten ohne DDM, bei denen $k_{\mathrm{t}}$ bis zu höheren Umsätzen deutlich fällt, kein Einsetzen des Geleffekts zu erkennen. Es gelangen allerdings keine Messungen bei Umsätzen größer $45 \%$, obwohl Inhomogenitäten bei diesen Messungen kein Problem darstellten. Jedoch sinkt bei höheren Umsätzen mit kleiner werdender Monomerkonzentration bei konstantem $k_{\mathrm{t}}$ der Umsatz pro Laserpuls. So verschlechtert sich das Signal-Rausch-Verhältnis immer weiter, bis schließlich unter diesen Bedingungen keine Messungen mehr möglich sind. Im Mittel über den gesamten Umsatzbereich ergibt sich ein Wert von $\log \left(k_{\mathrm{t}} /\left(\mathrm{L}_{\mathrm{mol}} \mathrm{m}^{-1} \cdot \mathrm{s}^{-1}\right)\right)=7.15$, was nur etwas kleiner als das bei kleinen Umsätzen ohne Zugabe von DDM bestimmte Ergebnis von $\log \left(k_{\mathrm{t}} /\left(\mathrm{L} \cdot \mathrm{mol}^{-1} \cdot \mathrm{s}^{-1}\right)\right)=7.39$ ist [6].

Eine Erklärung für das Fehlen eines Geleffekts liegt in der Produktion deutlich kleinerer Polymerketten. Somit wird die Viskosität der Reaktionsmischung herabgesetzt und bleibt 
auch bei höheren Umsätzen auf niedrigerem Niveau. Die Molmassenverteilung einer mit Zusatz von DDM hergestellten MMA-Probe ist in Abbildung 6.8 dargestellt.

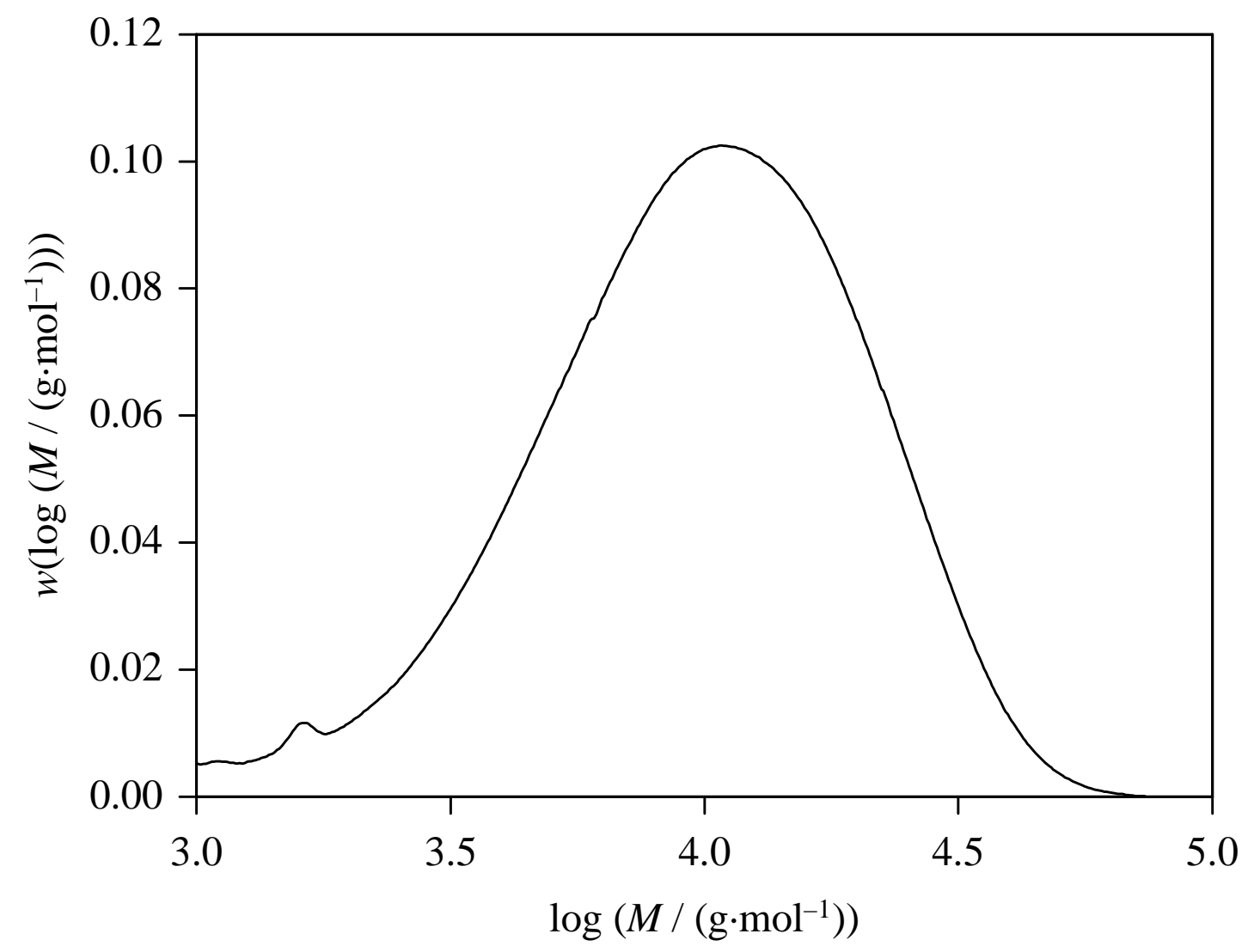

Abb. 6.8: Molmassenverteilung einer bei einem SP-PLP-Experiment bis $45 \%$ Umsatz mit Zusatz von DDM hergestellten Probe.

Mit dieser Probe wurden bei $40{ }^{\circ} \mathrm{C} / 1000$ bar SP-PLP-Experimente bis $45 \%$ Umsatz durchgeführt. Vor den laserinduzierten Experimenten erfolgte eine Vorpolymerisation bei $110{ }^{\circ} \mathrm{C} / 100$ bar bis $30 \%$ Monomerumsatz. Dabei wurde DDM mit $x_{\mathrm{DDM}}=0.025$ zugegeben. Diese Konzentration liegt im Bereich der maximalen von El Rezzi [13] verwendeten Menge bei der Bestimmung von Transferkonstanten von MMA-Homopolymerisationen in Substanz und überkritischem $\mathrm{CO}_{2}$. Es wurde eine hohe Konzentration an DDM gewählt, um einen deutlichen Effekt auf die Ergebnisse zu erreichen. Da außerdem DDM während der Reaktion verbraucht wird, ist eine große Menge an DDM sinnvoll, um zu gewährleisten, dass während des gesamten Reaktionsverlaufs eine effektive Molmassenregelung stattfindet. Die hier verwendete Konzentration an Mercaptan führte zu einer starken Verringerung der Molmasse auf einen Wert um $10^{4} \mathrm{~g} \cdot \mathrm{mol}^{-1}$. Das ist etwa zwei Größenordnungen kleiner als ohne Zugabe eines Kettenüberträgers (vgl. Abbildung 6.2). Auch bei hohen Umsätzen wurde das DDM 
nicht verbraucht, wie sich durch die Analyse entsprechender Proben zeigen ließ. Mit einer geringeren DDM-Konzentration könnte die Polymermolmasse gesteigert werden und vermutlich ergäbe sich dann auch wieder ein Geleffekt bei den Messungen. Allerdings reagiert das DDM bei zu kleiner Konzentration vor dem Ende der Polymerisation vollständig ab und es findet dann keine Molmassenregelung mehr statt. Dieses Problem ließe sich durch den Einsatz eines katalytischen Übertragungsagens lösen. So könnte die Molmasse bzw. Kettenlänge der terminierenden Radikale variabler eingestellt und der Einfluss auf $k_{\mathrm{t}}$ untersucht werden.

\subsection{Untersuchungen zur Kettenlängenabhängigkeit von $k_{\mathrm{t}}$ mit DMPA}

Ein optimales SP-PLP-Experiment beinhaltet die Verwendung eines Photoinitiators, bei dem beide Radikalfragmente effektiv und schnell mit einem Monomer reagieren und eine wachsende Kette starten. Die Untersuchungen in den Kapiteln 6 und 7 wurden mit DMPA (2,2-Dimethoxy-2-phenylacetophenon) als Initiator durchgeführt (siehe Abbildung 6.9).
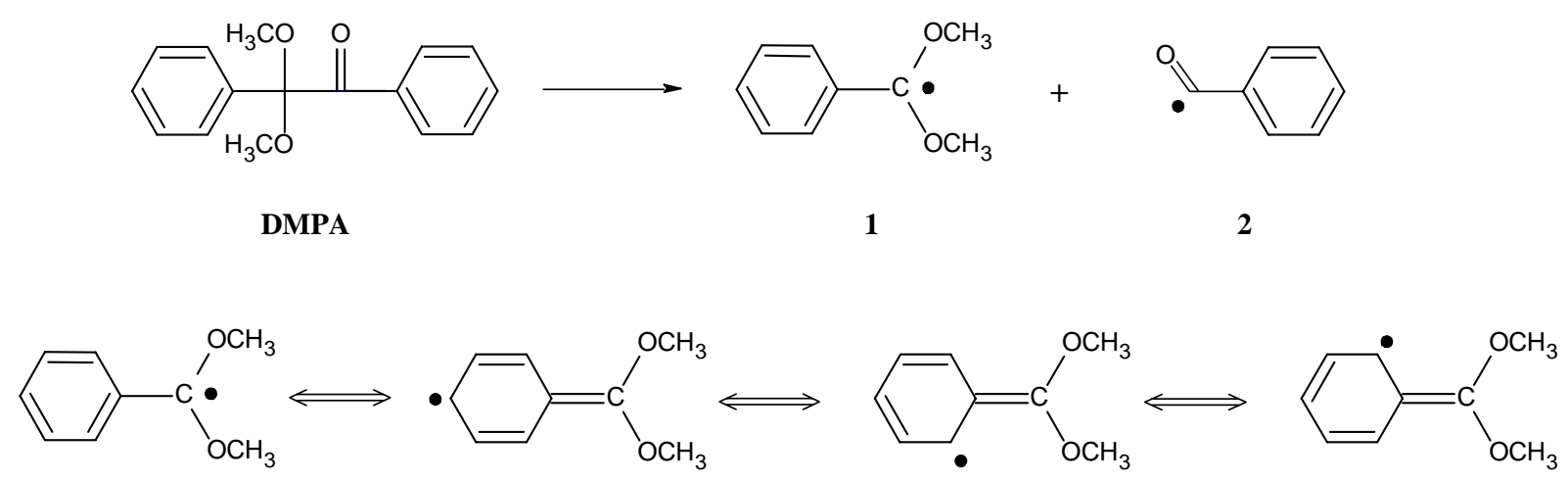

Abb. 6.9: Zerfallsschema des Photoinitiators DMPA (2,2-Dimethoxy-2-phenylacetophenon) (oben) und Resonanzstrukturen des Acetal-Fragments 1 (unten).

Wie in Abbildung 6.9 gezeigt, zerfällt DMPA in ein Acetal-Radikal 1 und ein BenzoylRadikal 2. Während 2 sehr effizient bezüglich der Addition an Monomermoleküle ist, reagiert 1 nicht nennenswert mit dem Monomer nach Auftreffen des Laserpulses.

Es wird aber vermutet, dass 1 mit Radikalen eine Terminierungsreaktion eingehen und somit wie ein Inhibitor wirken kann. Die geringe Aktivität bezüglich der Wachstumsreaktion von Radikal 1 wurde bereits von Fischer et al. [14] beschrieben und kürzlich durch MALDIExperimente von Davis und Mitarbeitern [15] bestätigt.

Dieses Verhalten kann dadurch erklärt werden, dass 1 über Resonanzstrukturen stabilisiert wird, was im unteren Teil von Abbildung 6.9 veranschaulicht ist. Durch die spezielle 
Eigenschaft von DMPA, sowohl initiierende als auch inhibierende Spezies zu erzeugen, ergibt sich bei SP-PLP-Experimenten eine Besonderheit. Mit unterschiedlicher Initiatorkonzentration unter sonst gleichen Bedingungen aufgenommene Signale kreuzen einander, so dass schließlich mit höherer Konzentration ein kleinerer Endumsatz resultiert [5]. Bei der Auswertung nach der idealen Polymerisationskinetik (Gleichung 3.6) ergibt sich für die höhere Konzentration ein größerer Wert von $k_{\mathrm{t}}$. In Abbildung 6.10 sind solche Signale von Buback et al. [5] für MA-Homopolymerisationen bei $40^{\circ} \mathrm{C} / 1000$ bar dargestellt.

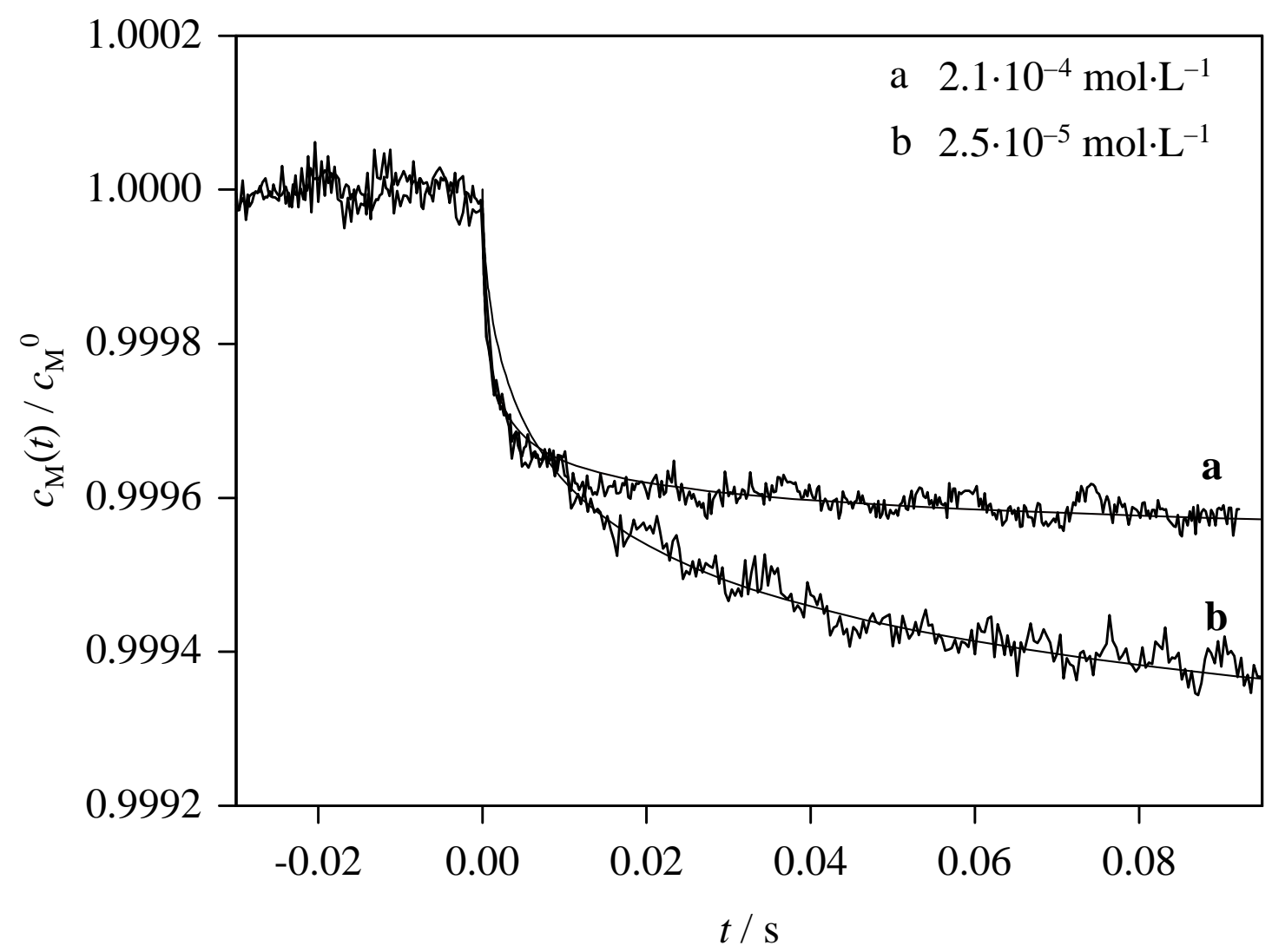

Abb. 6.10: Relative Monomerkonzentration als Funktion der Zeit für MA Homopolymerisationen bei $40{ }^{\circ} \mathrm{C} / 1000$ bar initiiert mit DMPA und verschiedenen Startradikalkonzentrationen [5]. Die durchgezogenen Linien veranschaulichen Anpassungen mit kettenlängenabhängigem $k_{\mathrm{t}}$.

Es konnte durch Modellierungen gezeigt werden, dass kreuzende Signale nur auftreten, wenn neben der Verwendung eines nicht idealen Photoinitiators auch eine Kettenlängenabhängigkeit des Terminierungsgeschwindigkeitskoeffizienten vorliegt [5]. Damit ergibt sich also ein Zugang zur Bestimmung dieser Kettenlängenabhängigkeit. Mit Hilfe des Programmpakets PREDICI ${ }^{\circledR}$ lässt sich ein entsprechendes kinetisches Schema aufstellen und 
an die Messkurven anpassen. Die Anpassungen sind in Abbildung 6.10 als durchgezogene Linien mit eingezeichnet. In dieser Arbeit sollte das Verfahren zur Ermittlung der Kettenlängenabhängigkeit von $k_{\mathrm{t}}$ aus sich kreuzenden Messsignalen für die MMAHopolymerisation bei höheren Monomerumsätzen angewendet werden. Dazu wurden Experimente mit der bereits beschriebenen Vorpolymerisation und verschiedenen DMPAKonzentrationen durchgeführt. Für die Modellierungen mit PREDICI ${ }^{\circledR}$ wurden anschließend Messsignale bei möglichst gleichem Monomerumsatz untersucht. Abbildung 6.11 zeigt zwei Signale, die bei etwa $17.5 \%$ Umsatz und $40{ }^{\circ} \mathrm{C} / 1000$ bar erhalten wurden.

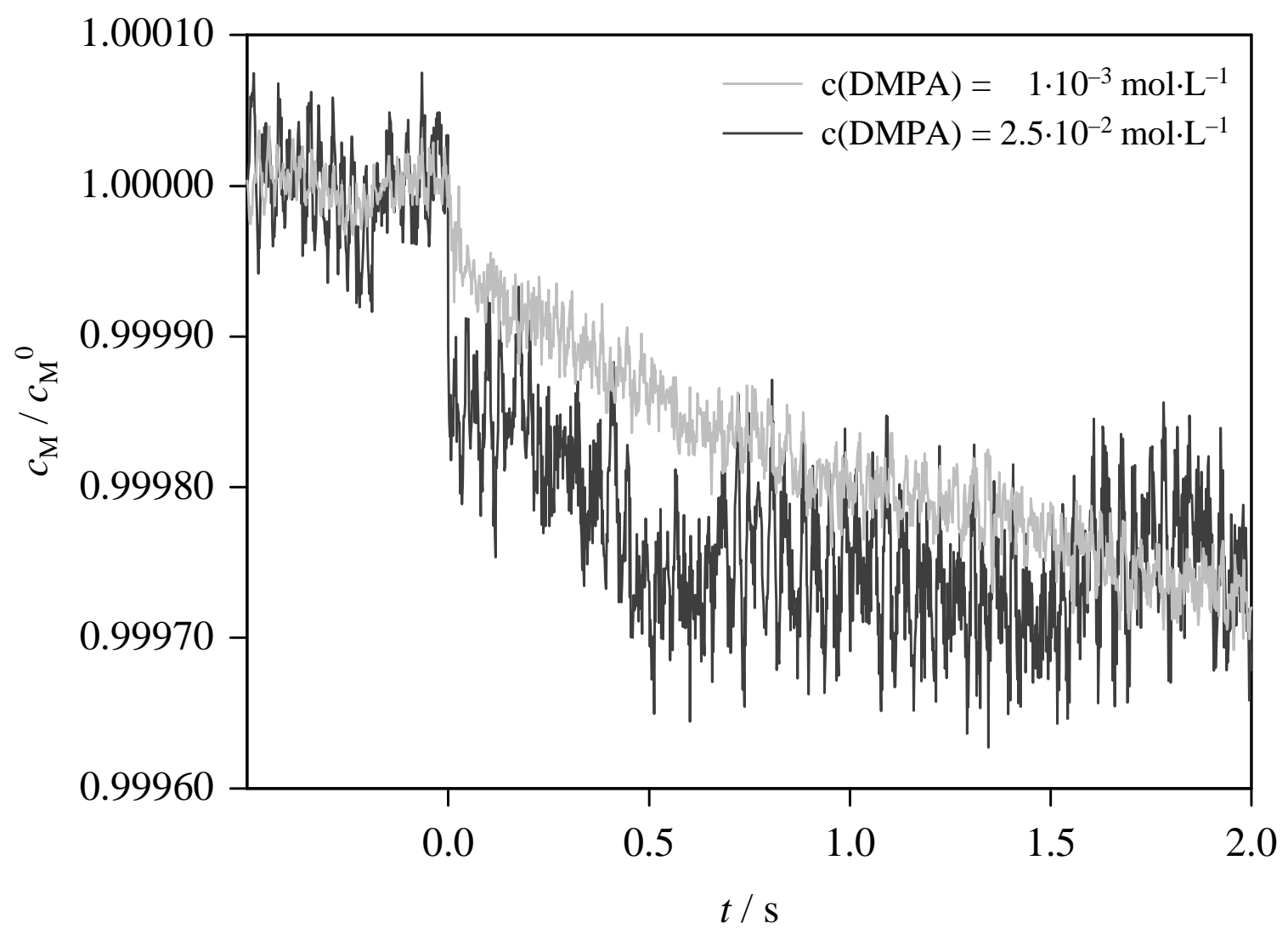

Abb. 6.11: Messsignale bei $40{ }^{\circ} \mathrm{C} / 1000$ bar und unterschiedlicher Initiatorkonzentration $(\sim 17.5 \%$ Monomerumsatz).

Eine ausführliche Beschreibung von Modellen zur Kettenlängenabhängigkeit von $k_{\mathrm{t}}$ erfolgt im Kapitel 8. Bei einem der Modelle ergibt sich ein Parameter $\alpha$ für das Ausmaß der Kettenlängenabhängigkeit. Kowollik [2] ermittelte für MMA bei $40^{\circ} \mathrm{C} / 1000$ bar einen Wert von $\alpha=0.15$, was in guter Übereinstimmung mit den Ergebnissen anderer Arbeitsgruppen $[16,17]$ ist. Diese Untersuchungen beziehen sich jedoch alle auf niedrige Monomerumsätze. 
Für die in Abbildung 6.11 gezeigten Signale wurde für $\alpha$ ein Wert von 0.18 bestimmt. Da mit einer Umsatzabhängigkeit von $\alpha$ zu rechnen ist, können für das hier vorgestellte Verfahren nur Paare von Signalen bei gleichem Umsatz untersucht werden. Außerdem führt eine Variation der Initiatorkonzentration zu unterschiedlichen Molmassen der gebildeten Polymerproben und wirkt sich so auf die Viskosität der Reaktionsmischung bei der Aufnahme der folgenden Signale aus. Um solche Einflüsse bei den Untersuchungen auszuschließen und möglichst gleiche Startbedingungen zu erreichen, sollten für die Analyse nur Signale ausgewählt werden, die am Anfang einer Messreihe aufgenommen wurden.

Diese Bedingungen schränken die Anzahl der für die Analyse infrage kommenden Signale stark ein. Somit konnten im Rahmen dieser Arbeit nur sehr wenige Signalpaarungen zur Modellierung herangezogen werden. Dabei ergaben sich für ebenso viele Paarungen kreuzende Signale wie nicht kreuzende Messkurven. Von den fünf sich kreuzenden Signalpaarungen ließ sich nur für zwei zufriedenstellende Anpassungen mit PREDICI ${ }^{\circledR}$ durchführen. Eines dieser beiden Paare ist das in Abbildung 6.11 dargestellte. Bei dem anderen Signalpaar konnte zwar eine gute Beschreibung der Messdaten mit dem Modell erreicht werden, wobei jedoch physikalisch nicht sinnvolle Parameter erhalten wurden.

Die Durchführung von SP-PLP-Messungen bei unterschiedlicher Initiatorkonzentration mit ansonsten völlig identischen Ausgangsbedingungen ist bei höheren Monomerumsätzen äußerst schwierig. Die in diesem Kapitel beschriebene Methode zur Bestimmung der Kettenlängenabhängigkeit von $k_{\mathrm{t}}$ für MMA mit DMPA als Photoinitiator ist als nicht empfehlenswert anzusehen. In Kapitel 8 wird eine alternative Prozedur vorgestellt, mit der die hier aufgetretenen Schwierigkeiten durch die Verwendung eines anderen Photoinitiators und die Anpassung einzelner Messsignale überwunden werden können. Auch bei den in Kapitel 8 dargestellten Untersuchungen wurde eine thermische Vorpolymerisation durchgeführt. Die Wahl des Photoinitiators hat keinen Einfluss auf die Homogenität der Reaktionsmischung, so dass ähnliche maximale Monomerumsätze erreicht wurden.

Die Untersuchungen in diesem Kapitel haben gezeigt, dass mit dem Hinzufügen einer Vorpolymerisation SP-PLP-Messungen reproduzierbar bis etwa $60 \%$ Monomerumsatz gelingen. Dabei ist ein möglichst hoher Druck bei den SP-PLP-Experimenten günstig. Durch die Verwendung eines zusätzlichen chemischen Initiators während der Vorpolymerisation lässt sich der zugängliche Umsatzbereich nicht weiter vergrößern. Wegen der beschriebenen Nachteile der verwendeten chemischen Initiatoren ist eine rein thermische Vorpolymerisation vorzuziehen. 
Die erhaltenen Ergebnisse für die Reaktionsdiffusionskonstante $C_{\mathrm{RD}}$ stimmen im Rahmen der Messungenauigkeit mit Literaturdaten überein. Über das Vorliegen einer Druckabhängigkeit von $C_{\mathrm{RD}}$ kann wegen der Unsicherheit in den Messdaten und weil nur bei einem Druck Experimente bis zu hohen Umsätzen durchgeführt werden konnten nicht entschieden werden.

Durch das Hinzufügen eines Kettenübertragungsagens und der damit verbundenen Verringerung der Polymermolmasse kann die Viskosität der Reaktionsmischung deutlich gesenkt werden. Bei MMA-Homopolymerisation führt das $\mathrm{zu}$ einem Ausbleiben des Geleffekts und einem $k_{\mathrm{t}}$ auf konstant hohem Niveau.

Eine Bestimmung der Kettenlängenabhängigkeit von $k_{\mathrm{t}}$ bei höheren Monomerumsätzen aus Messungen mit DMPA als Photoinitiator gelang nicht. Der Grund dafür lag in der großen Schwierigkeit, bei höheren Umsätzen Experimente unter bis auf die Initiatorkonzentration identischen Bedingungen durchzuführen.

\subsection{Literatur Kapitel 6}

[1] T. Junkers, Diplomarbeit, Göttingen 2002.

[2] C. Kowollik, Dissertation, Göttingen 1999.

[3] S. Beuermann, mündliche Mitteilung, 2002.

[4] M. Buback, B. Huckestein, G. T. Russell, Macromol. Chem. Phys. 1994, 195, 539.

[5] M. Buback, M. Busch, C. Kowollik, Macromol. Theory Simul. 2000, 9, 442.

[6] M. Buback, C. Kowollik, Macromolecules 1998, 31, 3211.

[7] M. Buback, D. Nelke, H.-P. Vögele, in Vorbereitung zur Veröffentlichung.

[8] S. Beuermann, Dissertation, Göttingen 1993.

[9] M. Buback, B. Degener, Macromol. Chem. 1993, 194, 2875.

[10] A. Gridnev, J. Polym. Sci, Polym. Chem. Ed. 2000, 38, 1753.

[11] J. P. A. Heuts, D. Kukulj, D. J. Foster, T. P. Davis, Macromolecules 1998, 31, 2894.

[12] R. A. Hutchinson, D. A. Paquet, J. H. McMinn, Macromolecules 1995, 28, 5655.

[13] V. El Rezzi, Dissertation, Göttingen 2001.

[14] H. Fischer, R. Baer, R. Hany, I. Verhoolen, M. Walbiner, J. Chem. Soc., Perkin Trans. 1990, $2,787$.

[15] P. Vana, T. P. Davis, C. Barner-Kowollik, J. Polym. Sci., Part A: Polym. Chem. 2002, $40,675$.

[16] H. K. Mahabadi, Macromolecules 1985, 18, 1319.

[17] O. F. Olaj, P. Vana, Macromol. Rapid Commun. 1998, 19, 433 und 533. 


\section{COPOLYMERISATIONEN}

In diesem Kapitel werden die Copolymerisationen der Methacrylat-Acrylat-Systeme Dodecylmethacrylat-Methylacrylat (DMA-MA) und Methylmethacrylat-Dodecylacrylat (MMA-DA) vorgestellt. Die Veränderung der Zusammensetzung der Monomermischung mit dem Umsatz und die Auswirkungen auf Wachstums- und Terminierungskinetik soll gezeigt werden. Es folgt eine Darstellung der Ergebnisse für $k_{\mathrm{t}}$ sowie die Beschreibung eines Terminierungsmodells und seine Anwendung bei den betrachteten Systemen. Dabei wird von einem kettenlängenunabhängigen $k_{\mathrm{t}}$ ausgegangen.

\subsection{Bestimmung von $k_{\mathrm{t}}$ aus SP-PLP-Experimenten}

Wie bereits in Kapitel 3 beschrieben, sind aus den SP-PLP-Experimenten bei Annahme einer idealen Polymerisationskinetik zunächst die gekoppelten Parameter $k_{\mathrm{t}} / k_{\mathrm{p}}$ zugänglich. Sie ergeben sich aus einer Anpassung von Gleichung 3.6 an die zeitaufgelöst gemessene Abnahme der Monomerkonzentration nach einem einzelnen Laserpuls. Durch Kombination mit einem unabhängig bestimmten $k_{\mathrm{p}}$ (aus PLP-SEC) lässt sich der (über alle Kettenlängen gemittelte) Terminierungsgeschwindigkeitskoeffizient $k_{\mathrm{t}}$ berechnen. Diese Methode zur Bestimmung von $k_{\mathrm{t}}$ wurde für zahlreiche Homopolymerisationen durchgeführt $[1,2,3,4,5]$.

Für Copolymerisationen sind in der Regel die Absorptionsmaxima im NIR-Spektrum sowie die Molarabsorptivitäten $B$ der beiden Monomere leicht verschieden. Kowollik hat gezeigt, dass trotzdem auch für Copolymerisationen entsprechende SP-PLP-Messungen durchgeführt und mit nur geringem Fehler ausgewertet werden können [6].

So lassen sich für Copolymerisationen nicht nur die Reaktionsbedingungen wie Druck und Temperatur variieren, sondern auch die Zusammensetzung der Comonomer-Mischung. Damit können weitere Erkenntnisse über den Terminierungsmechanismus gewonnen werden. Abbildung 7.1 zeigt Copolymerisationen von MMA-DA bei $40{ }^{\circ} \mathrm{C} / 1000 \mathrm{bar}$ und verschiedenen Mischungsverhältnissen [7], wobei $f_{\mathrm{MMA}}{ }^{0}$ der Molenbruch von MMA zu Beginn der Polymerisation ist. Aufgetragen sind die gekoppelten Parameter $k_{\mathrm{t}} / k_{\mathrm{p}}$ bis zu hohen Monomerumsätzen.

Bei niedrigen Umsätzen übertrifft $k_{\mathrm{t}} / k_{\mathrm{p}}$ für MMA um mehr als zwei Größenordungen den entsprechenden Wert für DA. Das hohe $k_{\mathrm{t}} / k_{\mathrm{p}}$ für MMA liegt sowohl an dem vergleichsweise großen $k_{\mathrm{t}}$ als auch an dem kleinen $k_{\mathrm{p}}$ und führt zu dem in Kapitel 6 ausführlich beschriebenen 
schlechten Signal-Rausch-Verhältnis. Für die hier diskutierten Copolymerisationen sind die Wachstums- und Terminierungsgeschwindigkeitskoeffizienten der entsprechenden Homopolymerisationen bei $40{ }^{\circ} \mathrm{C} / 1000$ bar in Abbildung 6.1 (Seite 36) eingetragen.

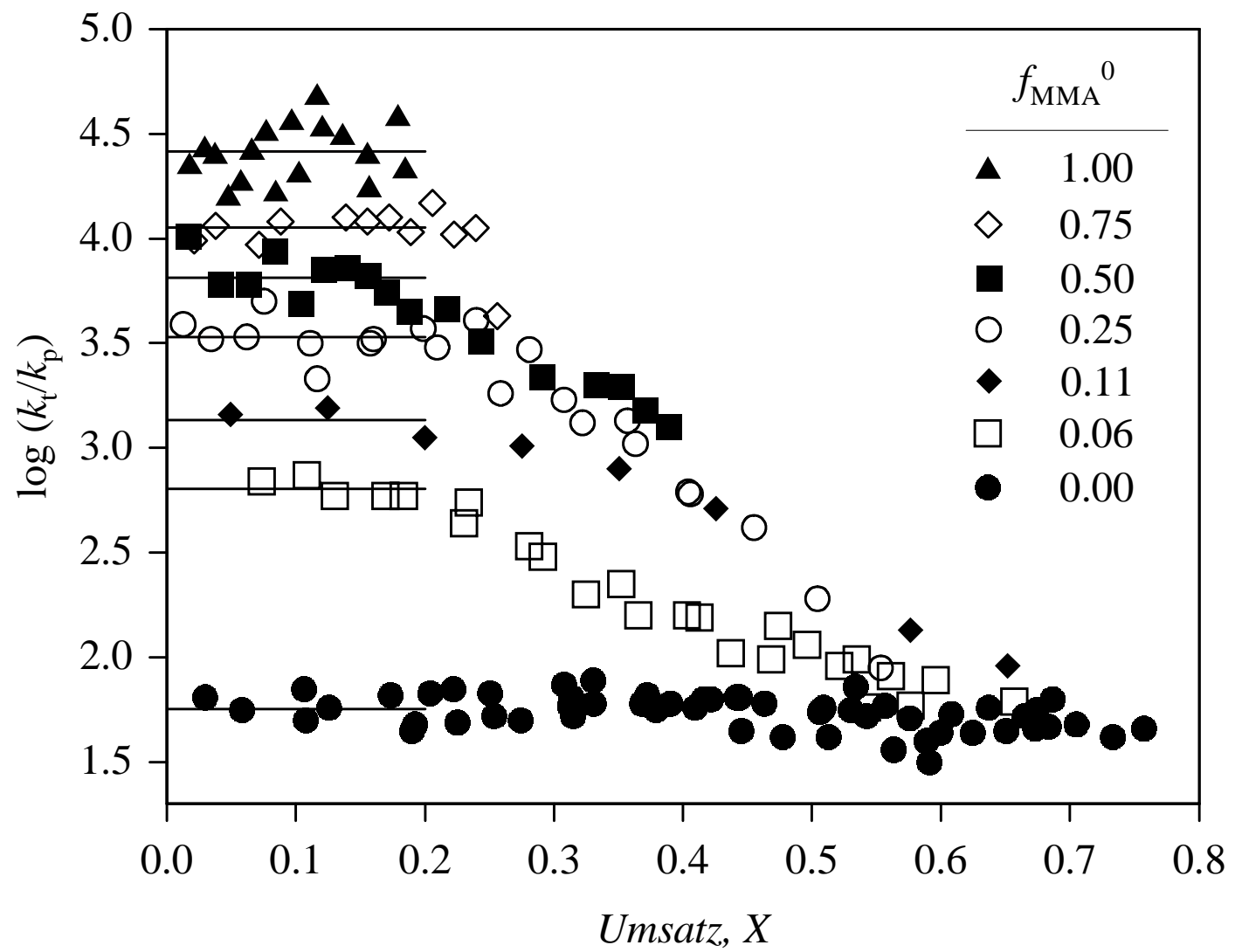

Abb. 7.1: $\log \left(k_{\mathrm{t}} / k_{\mathrm{p}}\right)$ für MMA-DA Copolymerisationen bei $40{ }^{\circ} \mathrm{C} / 1000$ bar als Funktion des Monomerumsatzes $X$. Mit $f_{\text {MMA }}{ }^{0}$ ist der Molenbruch von MMA in der Comonomer Mischung bezeichnet.

Das ungünstige Verhältnis von $k_{\mathrm{t}} \mathrm{zu} k_{\mathrm{p}}$ im Falle von MMA erklärt auch die große Streuung der als Dreiecke dargestellten Messwerte. Die Daten für reines MMA für Umsätze größer $20 \%$ stammen aus Experimenten mit Vorpolymerisationen (siehe Kapitel 6). Für alle in der Abbildung gezeigten Copolymerisationen ergibt sich bei niedrigen Umsätzen ein Plateaubereich mit nahezu konstanten $k_{\mathrm{t}} / k_{\mathrm{p}}$.

Da das chemisch kontrollierte $k_{\mathrm{p}}$ umsatzunabhängig sein sollte, ändert sich in diesem Anfangsbereich offensichtlich auch das diffusionskontrollierte $k_{\mathrm{t}}$ nur wenig. Der Plateaubereich ist sehr ausgedehnt für DA und eher schmal für MMA und Systeme mit hohem Anteil von MMA. Ähnliche Unterschiede im Plateauwert von $k_{\mathrm{t}} / k_{\mathrm{p}}$ wurden auch für das 
System MA-DA gefunden, wobei die Breite des Plateaus ebenfalls mit zunehmendem DA Gehalt ansteigt [8].

Bei höheren Umsätzen sind die Werte für $k_{\mathrm{t}} / k_{\mathrm{p}}$ im Gegensatz zum Anfangsbereich unabhängig von der Ausgangszusammensetzung der Monomermischung sehr ähnlich. Das deutet bei unterschiedlichem Umsatz auf verschiedene Arten der Diffusionskontrolle für $k_{\mathrm{t}}$ hin. Bei niedrigen Umsätzen wird davon ausgegangen, dass die Diffusion einzelner Segmente des Makroradikalknäuels die Terminierungsgeschwindigkeit bestimmt [9]. Wie in Kapitel 3 bereits beschrieben, wird für MMA bei höheren Umsätzen von einer Kontrolle durch Reaktionsdiffusion ausgegangen [10]. Für DA ist $k_{\mathrm{t}} / k_{\mathrm{p}}$ (und damit auch $k_{\mathrm{t}}$ ) innerhalb der Messgenauigkeit über den gesamten Umsatzbereich konstant. Es ist nicht möglich zu entscheiden, ob bei DA überall Segmentdiffusionskontrolle vorliegt oder ob bei den hohen Umsätzen die Reaktionsdiffusion wichtig wird.

In diesem Kapitel soll nur der Plateaubereich der Copolymerisationen diskutiert werden. In der Abbildung 6.1 stellen die horizontalen Linien jeweils den Mittelwert für $k_{\mathrm{t}} / k_{\mathrm{p}}$ bis $20 \%$ Umsatz dar.

Entsprechende Messungen für das System DMA-MA mit $f_{\mathrm{DMA}}{ }^{0}=1.0,0.75,0.25,0.05$ und 0.0 wurden ebenfalls bei $40{ }^{\circ} \mathrm{C} / 1000$ bar durchgeführt. Die $k_{\mathrm{t}} / k_{\mathrm{p}}$ Werte zeigen ein ähnliches Bild wie für MMA-DA, wobei $k_{\mathrm{t}} / k_{\mathrm{p}}$ von MA deutlich größer als bei DMA ist. Mit zunehmendem DMA Gehalt verbreitert sich der Plateaubereich bis zu $60 \%$ Umsatz bei reinem DMA.

$\mathrm{Zu}$ beiden hier besprochenen Systemen stehen $k_{\mathrm{p}}$ Daten bei den gleichen Messbedingungen von Buback et. al [11] zur Verfügung, von denen die für DMA-MA in Abbildung 7.2 dargestellt sind. 


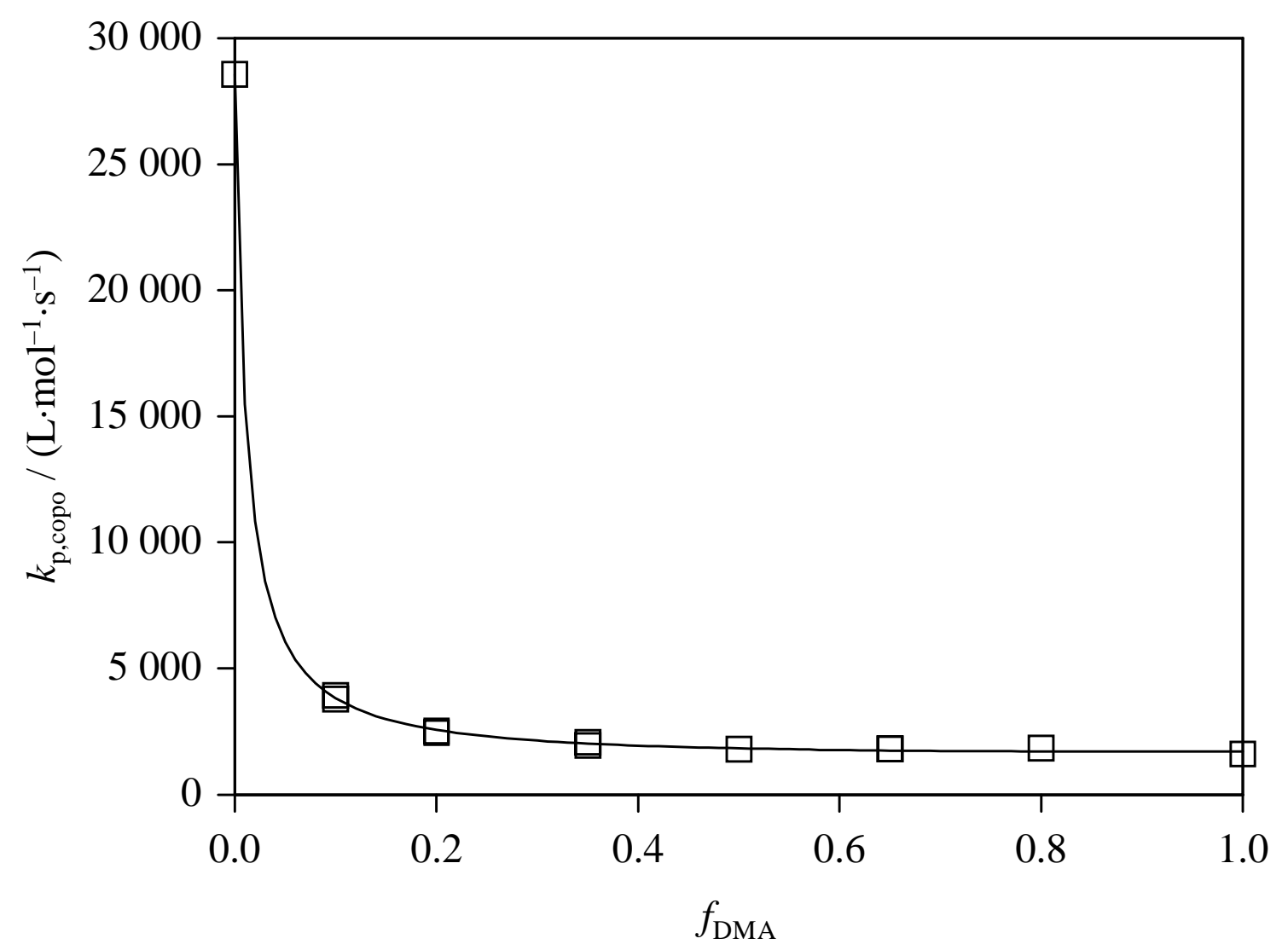

Abb. 7.2: Wachstumsgeschwindigkeitskoeffizient $k_{\mathrm{p}}$ für die DMA-MA Copolymerisation bei $40{ }^{\circ} \mathrm{C} / 1000$ als Funktion des Molenbruchs von DMA in der Comonomer Mischung, $f_{\text {DMA. }}$

Die Daten zeigen, dass $k_{\mathrm{p}}$ für MA um mehr als eine Größenordung über dem Wert für DMA liegt. Im Bereich zwischen $f_{\mathrm{DMA}}=0$ und 0.2 fällt $k_{\mathrm{p}}$ mit zunehmendem Anteil an DMA sehr stark ab und bleibt dann nahezu konstant. Gerade in diesem Bereich ist also $k_{\mathrm{p}}$ sehr empfindlich gegenüber kleinen Änderungen der Monomermischung. Da aber $k_{\mathrm{p}}$ direkt in die Berechnung von $k_{\mathrm{t}}$ eingeht, ist die Kenntnis der Molenbrüche der Monomere für jeden Messwert - auch bei höheren Umsätzen - notwendig.

Für Methacrylat-Acrylat Copolymerisationen ist jedoch bekannt, dass das Methacrylat im Allgemeinen bevorzugt in das Polymer eingebaut wird. In Abbildung 7.3 ist für vier Copolymerisationen bei niedrigen Umsätzen der Molenbruch des Methacrylats im Copolymer $F_{\text {Methacrylat }}$ gegen den Molenbruch im Monomerfeed $f_{\text {Methacrylat }}$ aufgetragen $[11,12]$. 


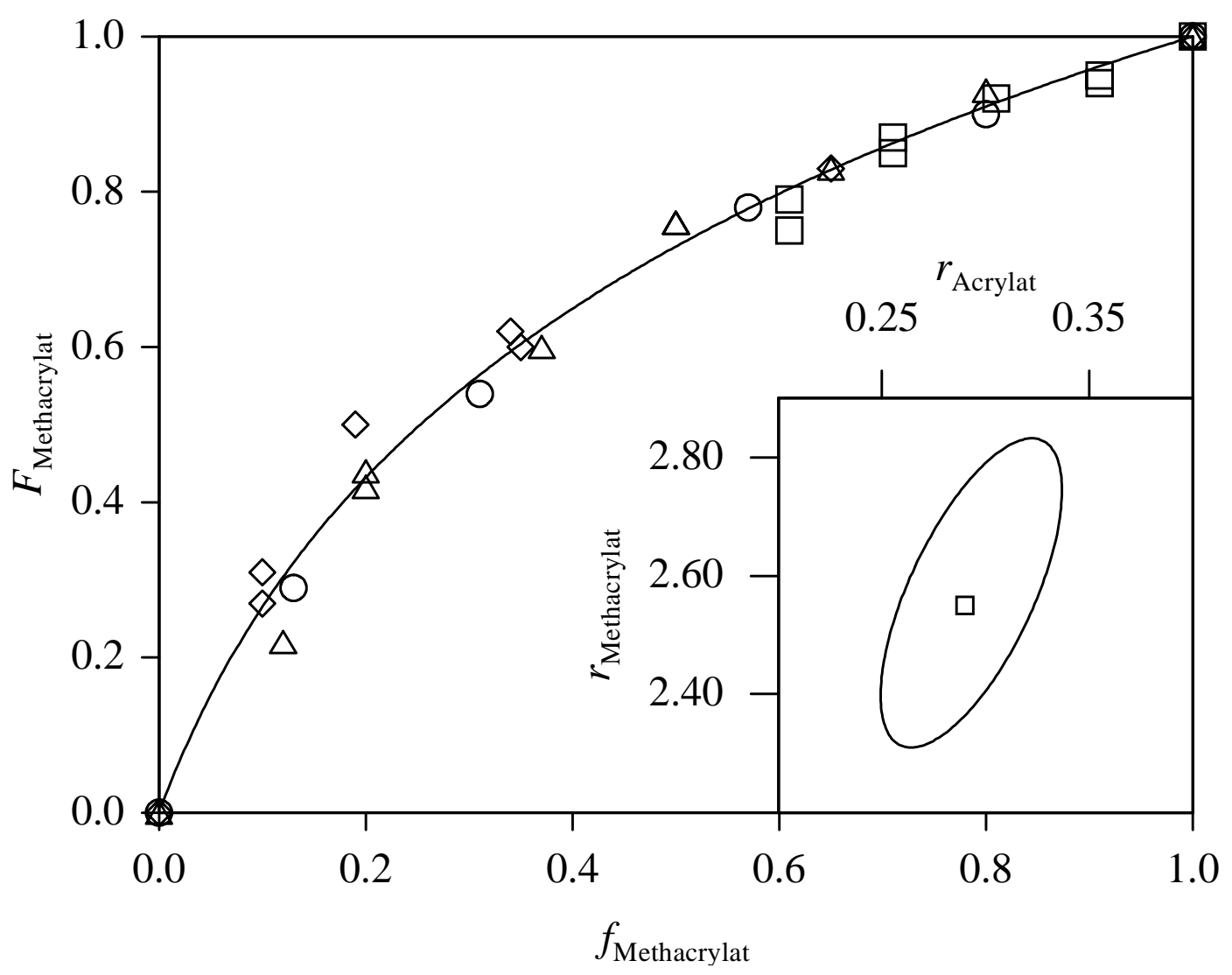

Abb. 7.3: Lewis-Mayo Auftragung für einige Copolymerisations-Systeme: MMA-BA (Kreise), DMA-MA (Rauten), MMA-DA (Dreiecke) und DMA-MA (Quadrate). Die durchgezogene Linie zeigt eine gemeinsame Anpassung der Lewis-Mayo Gleichung 7.1 an alle Datensätze. Alle Daten wurden bei $40{ }^{\circ} \mathrm{C} / 1000 \mathrm{bar}$ gemessen, bis auf MMA-BA $\left(50^{\circ} \mathrm{C} / 1\right.$ bar $)$.

Auch für die in der Abbildung gezeigten Systeme, inklusive DMA-MA und MMA-DA, kann ein erhöhter Anteil Methacrylat im Polymer gegenüber dem Monomergemisch nachgewiesen werden $[11,12]$. Die Linie stellt eine Anpassung der Lewis-Mayo Gleichung (7.1) [13] an die Messdaten dar.

$$
\frac{F_{1}}{F_{2}}=\frac{\mathrm{d} c_{\mathrm{M} 1}}{\mathrm{~d} c_{\mathrm{M} 2}}=\frac{f_{1} \cdot\left(r_{1} \cdot f_{1}+f_{2}\right)}{f_{2} \cdot\left(r_{2} \cdot f_{2}+f_{1}\right)} \quad \text { bzw. } \quad F_{1}=\frac{r_{1} \cdot f_{1}^{2}+f_{1} \cdot f_{2}}{r_{1} \cdot f_{1}^{2}+2 \cdot f_{1} \cdot f_{2}+r_{2} \cdot f_{2}^{2}}
$$

mit den Copolymerisationsparametern:

$$
r_{\mathrm{i}}=\frac{k_{\mathrm{pii}}}{k_{\mathrm{pij}}}
$$


Dabei ist folgendes kinetisches Schema zugrunde gelegt:

$$
\mathrm{R}-\mathrm{M}_{\mathrm{i}} \cdot+\mathrm{M}_{\mathrm{j}} \rightarrow \mathrm{R}-\mathrm{M}_{\mathrm{i}} \mathrm{M}_{\mathrm{j}} \cdot \quad k_{\mathrm{pij}} \quad \text { mit } \mathrm{i}, \mathrm{j}=1 \text { oder } 2
$$

Es wird also nur der Einfluss der letzten Monomereinheit der wachsenden Kette auf die Kinetik berücksichtigt (,terminal model“) [14].

Beim Fit von Gleichung 7.1 ergeben sich die Parameter $r_{1}$ und $r_{2}$ (in diesem Fall $r_{\text {Methacrylat }}$ und $\left.r_{\text {Acrylat }}\right)$. Die Daten aus Abbildung 7.3 von allen vier Systemen lassen sich gut mit einem Satz an Copolymerisationsparametern anpassen, wobei $r_{\text {Methacrylat }}=2.55$ und $r_{\text {Acrylat }}=0.29$ erhalten wird. Diese Werte sind mit einer berechneten $95 \%$ Konfidenzellipse ebenfalls in 7.3 dargestellt. Die genaue Art des Monomers scheint keinen Einfluss auf die $F_{\text {Methacrylat }}$ $f_{\text {Methacrylat }}$ Korrelation zu haben. Ebenso sind die Reaktionsbedingungen für den Zusammenhang von Copolymerzusammensetzung und Monomermischung nicht entscheidend, wie sich aus dem Vergleich der MMA-BA Daten $\left(50{ }^{\circ} \mathrm{C} / 1\right.$ bar $)$ mit den anderen Systemen $\left(40{ }^{\circ} \mathrm{C} / 1000\right.$ bar) zeigt. Über den gesamten Bereich der Zusammensetzungen liegt $F_{\text {Methacrylat }}$ deutlich über $f_{\text {Methacrylat }}$ Der Grund für diese Bevorzugung liegt wahrscheinlich in der größeren Stabilität von Radikalen, die nach Addition von einer Methacrylat Einheit an die wachsende Kette entstehen. Die $\alpha$-Methyl Gruppe des Methacrylats stabilisiert das Radikal durch eine Erhöhung der Elektronendichte.

Durch die bevorzugte Reaktion der wachsenden Ketten mit dem Methacrylat verarmt die Monomermischung an dieser Komponente zu höheren Umsätzen hin. Die aktuellen Werte von $f_{\text {Methacrylat }}$ unterscheiden sich so immer mehr von dem Molenbruch zu Beginn, $f_{\text {Methacrylat }}$. Um jedoch für die Auswertung der SP-PLP-Messungen ein korrektes $k_{\mathrm{p}}$ aus den in Abbildung 7.2 dargestellten Daten zu ermitteln, muss die aktuelle Zusammensetzung bekannt sein. Über die integrierte Form der Lewis-Mayo Gleichung (7.1), die Meyer-Lowry Gleichung [15], lässt sich die momentane Zusammensetzung für Untersuchungen bei höheren Umsätzen abschätzen. Für die Copolymerisationen von MMA-DA aus Abbildung 7.1 ist in Abbildung 7.4 die Entwicklung des aktuellen Molenbruchs von MMA, $f_{\text {MMA, als Funktion des }}$ Monomerumsatzes gezeigt. 


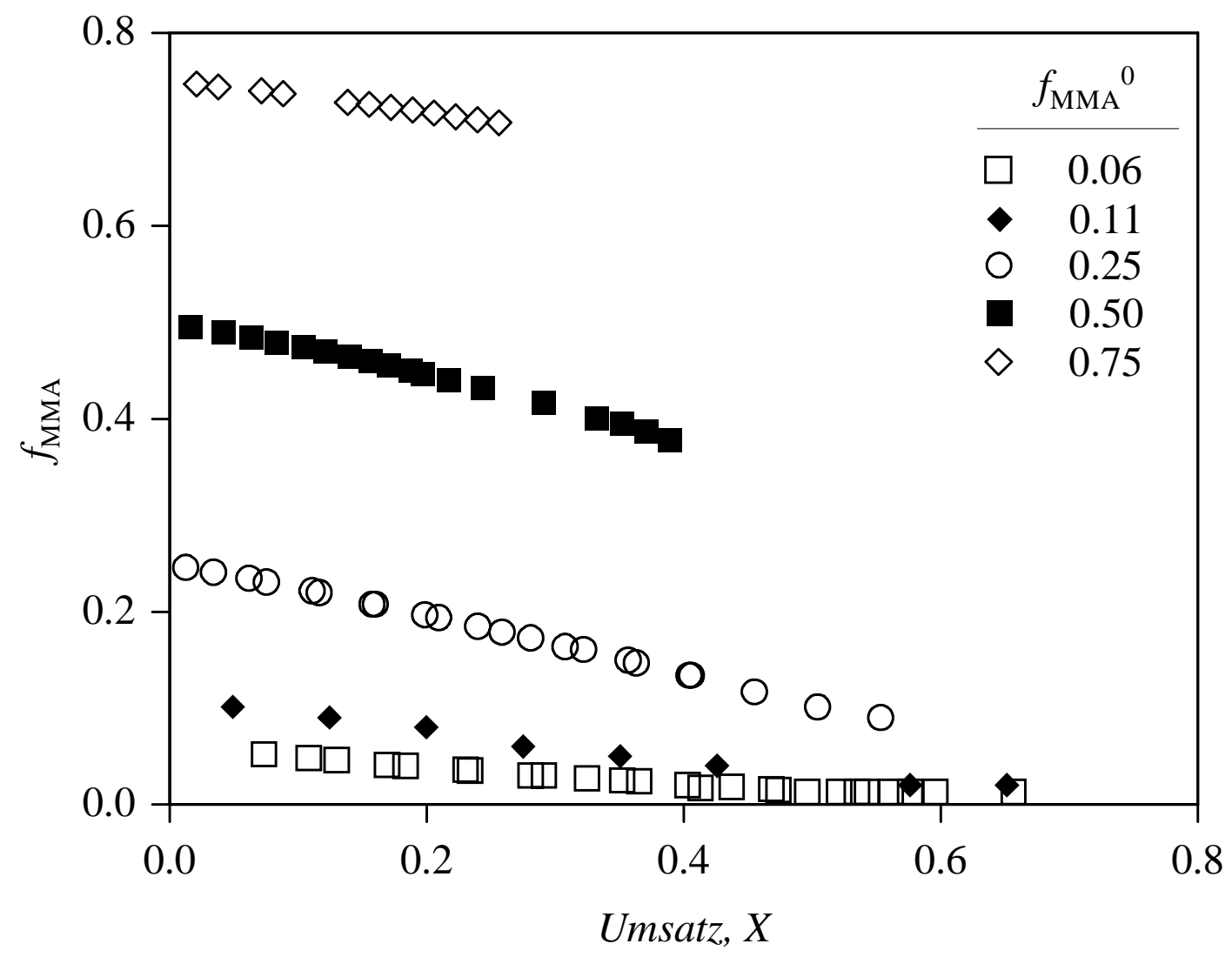

Abb. 7.4: Abgeschätzte Abnahme des MMA Molenbruchs, $f_{\mathrm{MMA}}$, während der Copolymerisationen von MMA-DA. Die Anfangszusammensetzungen sind identisch mit denen der Experimente dieses Abschnitts: $f_{\mathrm{MMA}}{ }^{0}=0.06,0.11,0.25,0.50$ und 0.75.

Die relative Änderung von $f_{\text {MMA }}$ ist besonders bedeutend für Mischungen mit niedrigem Anteil des Methacrylats. Die Abschätzung der aktuellen Zusammensetzung durch „terminal model“-Copolymerisationsparameter ist gerechtfertigt, da mit der Meyer-Lowry Gleichung nur die Prozedur zur Bestimmung der $r_{\mathrm{i}}$ umgekehrt wird.

Die Daten aus Abbildung 7.4 erlauben nun für jedes SP-PLP-Signal die Ermittlung des Molenbruchs beider Monomere und damit auch eines korrekten $k_{\mathrm{p}}$. Es sei an dieser Stelle noch erwähnt, dass diese aufwendige Auswertung der Daten bei Methacrylat-Methacrylat oder Acrylat-Acrylat Copolymerisationen nicht betrieben werden muss, da in diesen Fällen beide Monomere mit gleicher Wahrscheinlichkeit im Copolymer zu finden sind. Solche Copolymerisationen verlaufen fast ideal [8]. 


\subsection{Ergebnisse und Modellierungen}

Die aus dem SP-PLP-Experiment erhaltenen Ergebnisse für $k_{\mathrm{t}} / k_{\mathrm{p}}$ lassen sich in ein (über alle Kettenlängen gemitteltes) $k_{\mathrm{t}}$ umrechnen und dem dazugehörigen Molenbruch $f_{\text {Methacrylat }}$ zuordnen. Die so erhaltenen $k_{\mathrm{t}}$ Werte für die Plateaubereiche bis zu $20 \%$ Monomerumsatz der Systeme DMA-MA und MMA-DA werden in den Abbildungen 7.5 und 7.6 gezeigt.

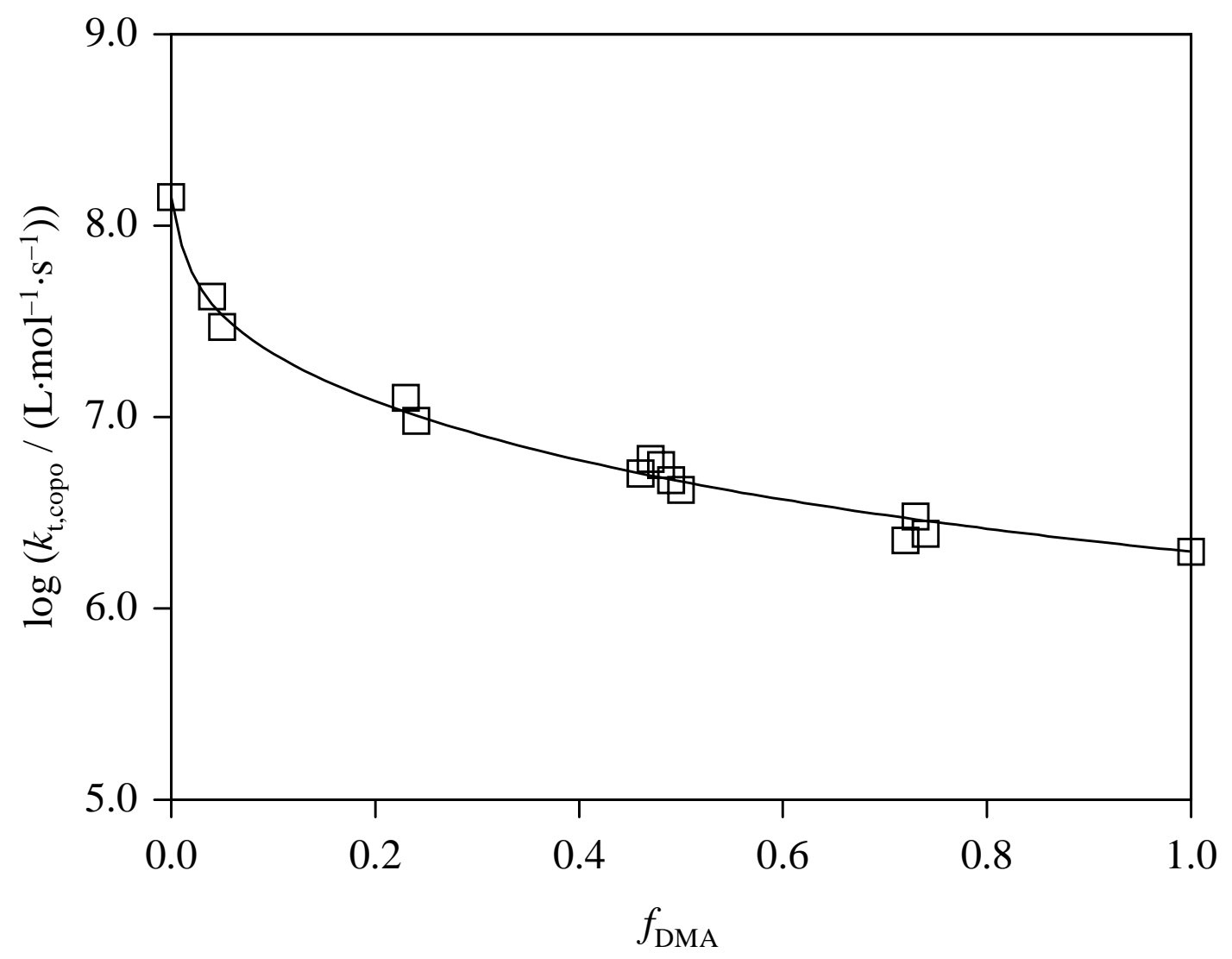

Abb. 7.5: (Mittlere) Terminierungsgeschwindigkeitskoeffizienten für den Plateaubereich der DMA-MA Copolymerisationen bei $40{ }^{\circ} \mathrm{C} / 1000$ bar aufgetragen gegen den aktuellen DMA-Molenbruch. Die durchgezogene Linie zeigt einen Fit mit dem unten beschriebenen ,penultimate model“ für $k_{\mathrm{t}}$ ( Gleichung 7.8).

In beiden Fällen werden Ansammlungen von Messpunkten erhalten, da sich in Abhängigkeit vom Umsatz bei gleicher Ausgangszusammensetzung $f_{\text {Methacrylat }}{ }^{0}$ unterschiedliche aktuelle Molenbrüche $f_{\text {Methacrylat }}$ ergeben. Die Korrelationen von $\log k_{\mathrm{t}}$ gegen $f$ zeigen für beide Copolymerisationen einen gekrümmten Verlauf im Gegensatz zum linearen Zusammenhang beim Acrylat-Acrylat System MA-DA [8] oder bei den Methacrylat-Methacrylat Systemen BMA-MMA [16] und DMA-MMA [16]. Ein linearer Verlauf wurde auch im Falle von 
DMA-DA erhalten [8] wobei hier die $k_{\mathrm{t}}$-Werte der entsprechenden Homopolymerisationen ohnehin gleich sind.

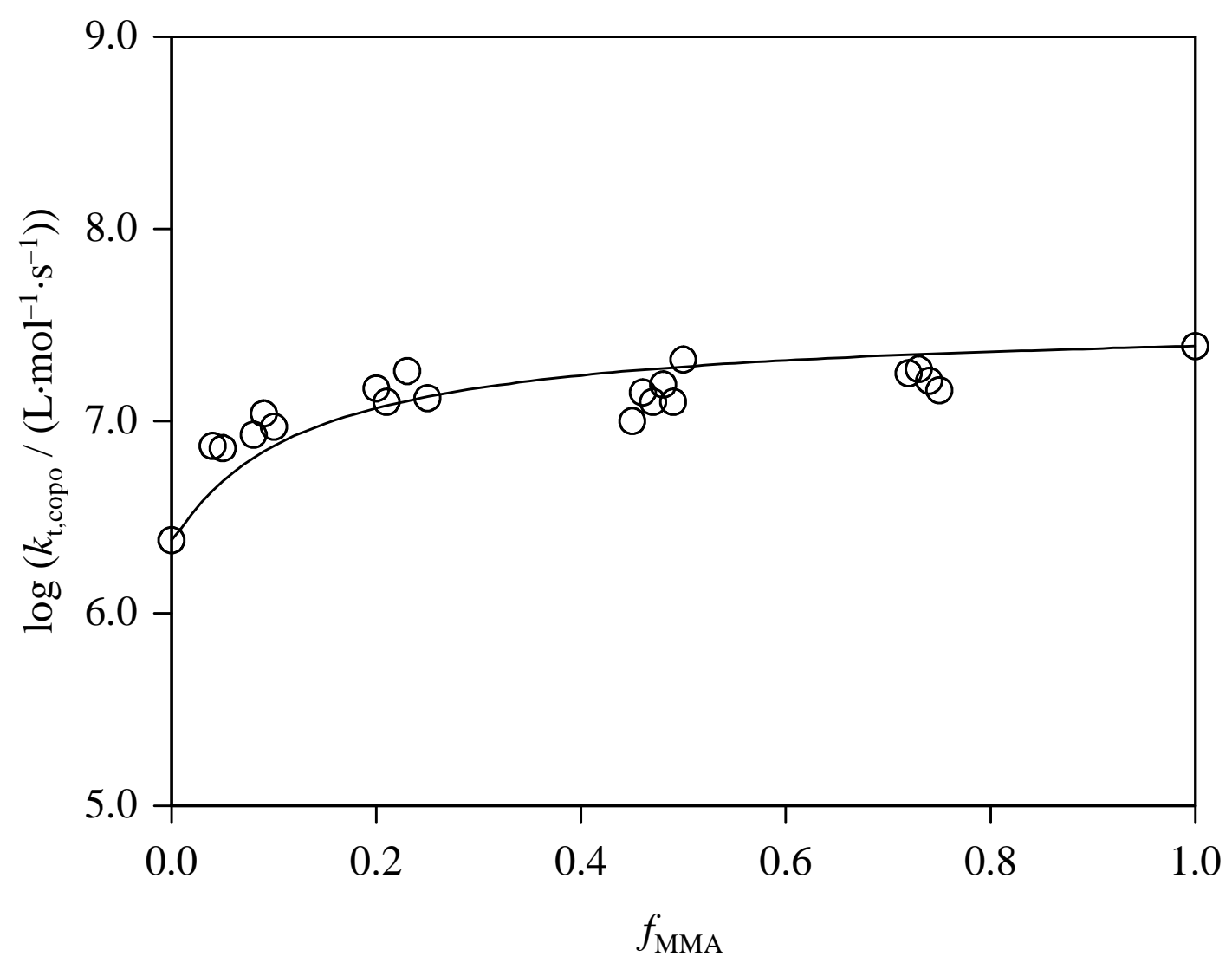

Abb. 7.6: (Mittlere) Terminierungsgeschwindigkeitskoeffizienten für den Plateaubereich der MMA-DA Copolymerisationen bei $40{ }^{\circ} \mathrm{C} / 1000$ bar aufgetragen gegen den aktuellen MMA Molenbruch. Die durchgezogene Linie zeigt einen Fit mit dem unten beschriebenen ,,penultimate model“ für $k_{\mathrm{t}}$ (Gleichung 7.8).

Trotz des Unterschiedes in der Korrelation zwischen $\log k_{\mathrm{t}}$ und $f$, gekrümmt oder linear, lassen sich die Daten für alle erwähnten Systeme, die hier betrachteten DMA-MA und MMA-DA eingeschlossen, mit demselben Terminierungsmodell beschreiben. Die Anpassungen sind in den Abbildungen 7.5 und 7.6 als durchgezogene Linien veranschaulicht. In Anbetracht der Unsicherheit der Bestimmung des direkt in $k_{\mathrm{t}}$ eingehenden $k_{\mathrm{p}}$, besonders bei niedrigem

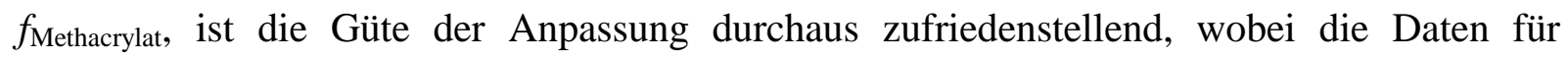
MMA-DA etwas schlechter beschrieben werden. Bei dem hier verwendeten Modell werden neben den letzten (,terminal“) auch die vorletzten (,penultimate“) Monomereinheiten der terminierenden Ketten berücksichtigt. Dieses Modell, das auf Russo und Munari [17, 18] zurückgeht, wurde von Fukuda et. al [19] weiterentwickelt und von Buback et. al [8] bei 
MA-DA und DMA-DA Copolymerisationen angewendet. Die wichtigen Gleichungen werden hier kurz aufgeführt. Ganz allgemein ergibt sich unter Betrachtung eines „penultimate“ Modells der folgende Ausdruck für den Terminierungsgeschwindigkeitskoeffizienten der Copolymerisation, $k_{\mathrm{t}, \mathrm{copo}}$ :

$$
k_{\mathrm{t}, \mathrm{copo}}=\sum_{\mathrm{l}=1}^{2} \sum_{\mathrm{k}=\mathrm{li}=1}^{2} \sum_{\mathrm{j}=1}^{2} \sum_{\mathrm{ij}} \cdot P_{\mathrm{kl}} \cdot k_{\mathrm{t} \mathrm{ij}, \mathrm{kl}}
$$

Hier sind $P_{\mathrm{ij}}$ und $P_{\mathrm{kl}}$ die Populationen der Radikale mit den letzten Monomereinheiten j und 1 und den vorletzten Einheiten i und k. Bei gegebenem Molenbruch eines Monomers werden diese Populationen aus den Wachstumsgeschwindigkeitskoeffizienten für die Homopolymerisation $k_{\mathrm{ii}}$, den Copolymerisationsparametern (,terminal“" und ,penultimate“) $r_{\mathrm{i}}$ und $r_{\mathrm{ii}}$, den (,penultimate“) Radikal-Copolymerisationsparametern $s_{\mathrm{i}}=k_{\mathrm{pjii}} / k_{\mathrm{piii}}$ sowie den Populationen nach dem ,terminal model“ $P_{\mathrm{i}}$ und $P_{\mathrm{j}}$ entsprechend den Gleichungen 7.3 und 7.4 abgeschätzt [20]:

$$
\begin{gathered}
P_{1}=1-P_{2}=\frac{\frac{\overline{r_{1}} \cdot f_{1}}{\overline{k_{11}}}}{\frac{\overline{r_{1}} \cdot f_{1}}{\overline{k_{11}}}+\frac{\overline{r_{2}} \cdot f_{2}}{\overline{k_{22}}}} \\
P_{11}=P_{1}-P_{21}=\frac{r_{11} \cdot f_{1} \cdot P_{1}}{r_{11} \cdot f_{1}+\frac{f_{2}}{s_{1}}}
\end{gathered}
$$

Der Querstrich über einigen Größen in 7.3 bedeutet, dass diese Variablen wiederum von der Mischungszusammensetzung abhängen. Sie lassen sich wie folgt berechnen [21]:

$$
\begin{aligned}
& \overline{r_{\mathrm{i}}}=r_{\mathrm{ji}} \cdot \frac{r_{\mathrm{ii}} \cdot f_{\mathrm{i}}+f_{\mathrm{j}}}{r_{\mathrm{ji}} \cdot f_{\mathrm{i}}+f_{\mathrm{j}}} \\
& \overline{k_{\mathrm{pii}}}=k_{\mathrm{piii}} \cdot \frac{r_{\mathrm{ii}} \cdot f_{\mathrm{i}}+f_{\mathrm{j}}}{r_{\mathrm{ii}} \cdot f_{\mathrm{i}}+f_{\mathrm{j}} / s_{\mathrm{i}}}
\end{aligned}
$$

Aus der Gleichung 7.2 ergeben sich insgesamt zehn „penultimate“ Terminierungsgeschwindigkeitskoeffizienten $k_{\mathrm{t}} \mathrm{ij,kl}$. Um diese große Anzahl zu reduzieren, werden die sogenannten ,unlike“-Terminierungen $k_{\mathrm{t}} \mathrm{jj}, \mathrm{kl}$ (mit $\mathrm{ij} \neq \mathrm{kl}$ ) durch eine geometrische Mittelung der ,like“-Terminierungen $k_{\mathrm{t} \mathrm{ij}, \mathrm{ij}}$ und $k_{\mathrm{t} \mathrm{kl}, \mathrm{kl}}$ angenähert $[8,19]$ : 


$$
k_{\mathrm{t} \mathrm{ij}, \mathrm{kl}}=\left(k_{\mathrm{t} \mathrm{j}, \mathrm{ij}} \cdot k_{\mathrm{t} \mathrm{kl}, \mathrm{kl}}\right)^{0.5}
$$

Einsetzen von 7.7 in 7.2 ergibt schließlich:

$$
k_{\mathrm{t}, \mathrm{copo}}^{0.5}=k_{\mathrm{t} 11,11}^{0.5} \cdot P_{11}+k_{\mathrm{t} 21,21}^{0.5} \cdot P_{21}+k_{\mathrm{t} 12,12}^{0.5} \cdot P_{12}+k_{\mathrm{t} 22,22}^{0.5} \cdot P_{22}
$$

wobei $k_{\mathrm{t} 11,11}$ und $k_{\mathrm{t} 22,22}$ die Terminierungsgeschwindigkeitskoeffizienten für die entsprechenden Homopolymerisationen sind. Dieses Modell wurde neben den schon erwähnten Systemen MA-DA [8] und DMA-DA [8] bereits für andere Copolymerisationen wie Styrol-Diethylfumarat [22] erfolgreich angewendet.

In einigen Fällen hat sich gezeigt, dass bei der Anpassung von Gleichung 7.8 an die Messdaten $k_{\mathrm{t} 21,21}=k_{\mathrm{t}} 12,12$ resultiert. Für die Fits aus den Abbildungen 7.5 und 7.6 wurde angenommen, dass dies auch hier gilt. Die Populationen der ,penultimate“ Radikale $P_{\mathrm{ij}}$ lassen sich mit den Gleichungen 7.3 und 7.4 bestimmen. Dabei wurden die Copolymerisationsparameter $r_{\text {Methacrylat }}=2.55$ und $r_{\text {Acrylat }}=0.29$ benutzt, die gemäß der Diskussion zu Abbildung 7.3 für viele Methacrylat-Acrylat Systeme gültig sind. Die RadikalCopolymerisationsparameter wurden mit $s_{\text {Methacrylat }}=2$ und $s_{\text {Acrylat }}=0.5$ abgeschätzt, was nahe den für MMA-BA bestimmten Werten ist [12].

Es ergibt sich $k_{\mathrm{t} 21,21}=k_{\mathrm{t}} 12,12=3.08 \cdot 10^{7} \mathrm{~L} \cdot \mathrm{mol}^{-1} \cdot \mathrm{s}^{-1}$ für DMA-MA und $2.55 \cdot 10^{6} \mathrm{~L} \cdot \mathrm{mol}^{-1} \cdot \mathrm{s}^{-1}$ für MMA-DA. Für beide Copolymerisationen liegen diese „like“-Terminierungsgeschwindigkeitskoeffizienten der Radikale mit unterschiedlicher letzter und vorletzter Monomereinheit wie erwartet zwischen den entsprechenden Koeffizienten für die Homopolymerisationen $k_{\mathrm{t} 11,11}$ und $k_{\mathrm{t} 22,22}$. Die Fitprozedur lässt sich auch ohne die Einschränkung $k_{\mathrm{t} 21,21}=k_{\mathrm{t} 12,12}$ durchführen und bringt noch etwas bessere Anpassungen an die Messdaten. Dabei resultieren jedoch Werte für die „like“-Koeffizienten, die sich nicht mehr zwischen den $k_{\mathrm{t}} 11,11$ und $k_{\mathrm{t}} 22,22$ befinden. Ein solches Ergebnis erscheint physikalisch nicht sinnvoll.

Zusammenfassend ergibt sich also, dass bei Methacrylat-Acrylat-Copolymerisationen mit einer Abhängigkeit der Zusammensetzung der Monomermischung vom Umsatz zu rechnen ist. Daraus resultiert auch eine Abhängigkeit von $k_{\mathrm{p}}$ vom Monomerumsatz, was bei der Bestimmung von $k_{\mathrm{t}}$ aus SP-PLP-Messungen berücksichtigt werden muss. Die Beschreibung des Terminierungsgeschwindigkeitskoeffizienten für Copolymerisationen gelingt dann bei sehr verschiedenen Systemen mit demselben ,penultimate“ Modell. 


\subsection{Literatur Kapitel 7}

[1] M. Buback, H. Hippler, J. Schweer, H.-P. Vögele, Makromol. Chem., Rapid Commun. 1986, 7, 261.

[2] B. Degener, Dissertation, Göttingen 1992.

[3] C. Kurz, Dissertation, Göttingen 1995.

[4] M. Buback, C. Kowollik, Macromolecules 1998, 31, 3221.

[5] M. Buback, C. Kowollik, C. H. Kurz, A. Wahl, Macromol. Chem. Phys. 2000, 201, 464.

[6] C. Kowollik, Dissertation, Göttingen 1999.

[7] M. Buback, A. Feldermann, Aust. J. Chem. 2002, 55, 475.

[8] M. Buback, C. Kowollik, Macromolecules 1999, 32, 1445.

[9] S. W. Benson, A. M. North, J. Am. Chem. Soc. 1962, 84, 935.

[10] G. V. Schulz, Z. Phys. Chem. (München) 1956, 8, 290.

[11] M. Buback, A. Feldermann, C. Barner-Kowollik, I. Lací kMacromolecules 2001, 34, 5439.

[12] R. A. Hutchinson, J. H. McMinn, D. A. Paquet Jr., S. Beuermann, C. Jackson, Ind. Eng. Chem. Res. 1997, 36, 1103.

[13] F. R. Mayo, F. M. Lewis, J. Am. Chem. Soc. 1954, 66, 1944.

[14] E. Merz, T. Alfrey, G. Goldfinger, J. Polym. Sci. 1946, 1, 75.

[15] G. Odian, Principles of Polymerization, 3rd edition,Wiley, New York, 1991.

[16] K. F. O’Driscoll, K. Ito, J. Polym. Sci. 1979, 17, 3913.

[17] S. Russo, S. Munari, J. Macromol. Sci. Chem. 1968, 2, 1321.

[18] G. Bonta, B. M. Gallo, S. Russo, J. Chem. Soc., Faraday Trans. 1 1975, 71, 1721.

[19] T. Fukuda, N. Ide, Y.-D. Ma, Macromol. Symp. 1996, 111, 305.

[20] T. Fukuda, K. Kubo, Y.-D. Ma, Prog. Polym. Sci. 1992, 17, 875.

[21] T. Fukuda, Y.-D. Ma, H. Inagaki, Macromolecules 1985, 18, 17.

[22] Y.-D. Ma, K.-S. Sung, Y. Tsujii, T. Fukuda, Macromolecules 2001, 34, 4749. 


\section{KETTENLÄNGENABHÄNGIGKEIT VON $\boldsymbol{k}_{\mathrm{t}}$}

Bei der SP-PLP-Technik wird eine schmale Größenverteilung der Radikale erreicht, wobei die Kettenlänge linear mit der Zeit nach dem Laserpuls (bei $t=0$ ) zunimmt. Diese Besonderheit erlaubt die Bestimmung der Kettenlängenabhängigkeit der Terminierungsreaktion. Allerdings ist die enge Poisson-Verteilung auf die frühen Aufnahmezeiten, in denen eine Verbreiterung durch Transfer zum Monomer oder Polymer noch nicht wesentlich auftritt, begrenzt. In diesem Kapitel werden Details zur Auswertung von SP-PLP-Signalen bezüglich einer Kettenlängenabhängigkeit von $k_{\mathrm{t}}$ dargestellt und die erhaltenen Ergebnisse diskutiert. Dabei soll zunächst der bei diesem SP-PLP-Experimenten verwendete Photoinitiator vorgestellt werden.

\subsection{Auswahl des Photoinitiators}

Neben der Beschränkung auf kurze Analysezeiträume zeichnet sich ein optimales SP-PLPExperiment durch die Verwendung eines Photoinitiators aus, bei dem beide Radikalfragmente mit der gleichen Effektivität und Geschwindigkeit durch Reaktion mit dem Monomer eine wachsende Kette starten. Wie bereits in Kapitel 6 erwähnt, erfüllt der bei den in den Kapiteln 6 und 7 vorgestellten Untersuchungen verwendete Photoinitiator DMPA diese Voraussetzungen nicht. Während eines der Radikalfragmente sehr effizient an Monomermoleküle addiert, nimmt das andere Fragment nur an Terminierungsreaktionen teil und wirkt wie ein Inhibitor [1, 2]. Diese Nichtidealität von DMPA lässt sich, wie in Kapitel 6 beschrieben, allerdings auch für eine Bestimmung der Kettenlängenabhängigkeit von $k_{\mathrm{t}}$ ausnutzen [3]. Es kann es bei diesem Verfahren jedoch zu einigen Schwierigkeiten kommen. So muss eine recht große Anzahl an Parametern angepasst oder abgeschätzt werden. Zudem ist die Messung von mehreren Signalen unter - mit Ausnahme der DMPA-Konzentration - gleichen experimentellen Bedingungen schwierig, besonders bei mittleren und höheren Monomerumsätzen. Aus diesen Gründen ist es wünschenswert, bei den Untersuchungen einen Photoinitiator zu verwenden, der in zwei Radikale von gleicher und hoher Aktivität bezüglich der Addition an Monomeres zerfällt, und die Kettenlängenabhängigkeit direkt aus jedem einzelnen Signal zu bestimmen. Vor der Vorstellung eines solchen Initiators sollte allerdings noch erwähnt werden, dass die Inhibierung durch das Radikalfragment vom Methoxy-Typ (siehe Abbildung 6.9, Seite 49) des DMPA die Bestimmung von $k_{\mathrm{t}}$ bei niedrigen Initiatorkonzentrationen nur geringfügig beeinflusst. Die in den Kapiteln 6 und 7 vorgestellten 
Ergebnisse wurden unter solchen Bedingungen erhalten. Das dort gezeigte $k_{\mathrm{t}}$ aus Experimenten mit niedriger Initiatorkonzentration kann als über alle Kettenlängen gemittelter Terminierungsgeschwindigkeitskoeffizient angesehen werden.

Neben dem Zerfall in zwei aktive Spezies gibt es noch weitere Anforderungen an einen optimalen Photoinitiator für SP-PLP-Experimente. Dazu gehört eine kurze Lebensdauer im angeregten Zustand, eine gute Löslichkeit in den gängigen Monomeren und ein großer Absorptionskoeffizient für Laserlicht der verwendeten Wellenlänge. Bei Untersuchungen mit verschiedenen Photoinitiatoren fand Külpmann, dass MMMP ( $\alpha$-Methyl-4-(methylmercapto)- $\alpha$-morpholinopropiophenon) schnell in zwei nahezu gleich reaktive Radikale zerfällt [4]. Das Zerfallsschema dieses Photoinitiators ist in Abbildung 8.1 gezeichnet.

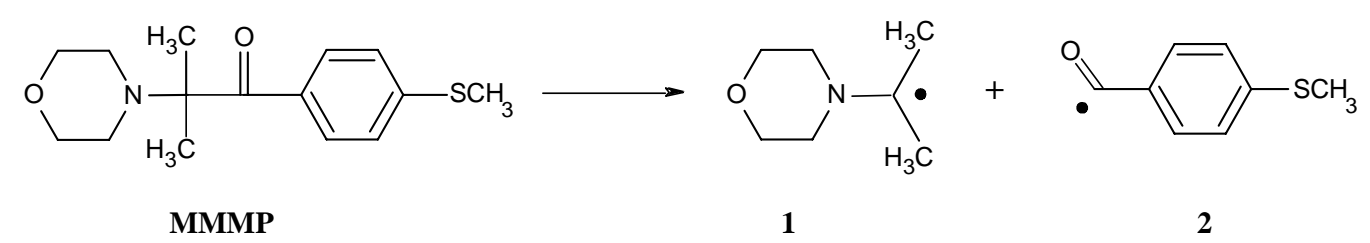

Abb. 8.1: Zerfallsschema des Photoinitiators MMMP ( $\alpha$-Methyl-4-(methylmercapto)$\alpha$-morpholinopropiophenon).

Als Beleg für die Idealität von MMMP dienen zum einen Endgruppenanalysen von Polymerproben, die mit diesem Initiator hergestellt wurden [5]. Dabei hat sich gezeigt, dass beide entstehenden Radikale mit nahezu gleicher Häufigkeit im Polymer zu finden sind. Deshalb ist von einer sehr ähnlichen Reaktivität der Fragmente 1 und 2 (vgl. Abbildung 8.1) auszugehen.

Zum anderen können Experimente mit verschiedenen Startkonzentrationen analog zu denen aus Abbildung 6.10 (Seite 50) durchgeführt werden. In Abbildung 8.2 sind entsprechende Signale von Külpmann [4] für MA-Homopolymerisationen bei $40^{\circ} \mathrm{C} / 2000$ bar initiiert mit MMMP gezeigt. Die Messkurven für verschiedene Radikalkonzentrationen kreuzen sich im Gegensatz zu den Untersuchungen mit DMPA (vgl. Abbildung 6.10, Seite 50) nicht. Die Auswertung nach idealer Polymerisationskinetik (Gleichung 3.6, als durchgezogene Linie gezeichnet) liefert in diesem Falle bei verschiedenen Startradikalkonzentrationen stets den gleichen Wert für $k_{\mathrm{t}}$. Dies sind starke Indizien dafür, dass bei diesem Initiator beide entstehenden Radikale zur Initiierung beitragen. Eine genauere Darstellung der Untersuchungen findet sich bei Külpmann [4]. 


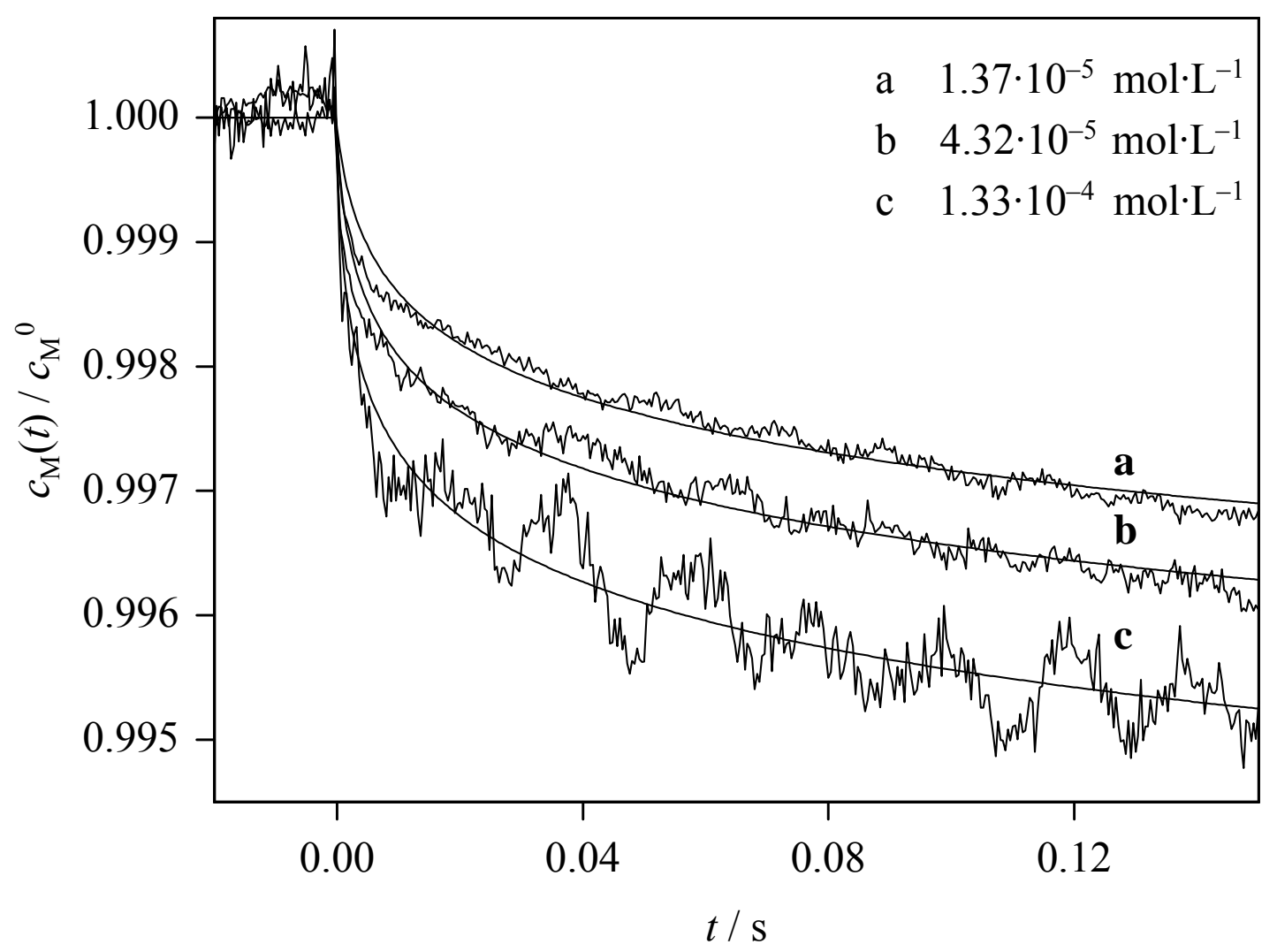

Abb. 8.2: Relative Monomerkonzentration als Funktion der Zeit für MA-Homopolymerisationen bei $40{ }^{\circ} \mathrm{C} / 1000$ bar initiiert mit MMMP und verschiedenen Startradikalkonzentrationen sowie Anpassungen nach idealer Polymerisationskinetik [4].

Durch die Aktivität beider Radikale vereinfacht sich die Auswertung von SP-PLP-Signalen bezüglich einer Kettenlängenabhängigkeit von $k_{\mathrm{t}}$ erheblich. Deshalb wurden die im Folgenden beschriebenen Experimente zur detaillierten Betrachtung der Kettenlängenabhängigkeit der Terminierungsreaktion mit MMMP durchgeführt.

\subsection{Auswertung der Messsignale}

Bei der Analyse der Signale sollen zur Vereinfachung der Auswertung Transferreaktionen nicht betrachtet werden. Dazu muss man sich allerdings auf kurze Aufnahmezeiten nach Auslösen des Laserpulses bei $t=0$ beschränken. Es wird hier aufgrund der Koeffizienten für den Transfer zum Monomer [6,7] davon ausgegangen, dass bis zu einer Zeit von $0.1 \mathrm{~s}$ für Acrylate und $0.5 \mathrm{~s}$ für Methacrylate der Einfluss von Kettenübertragung auf das Monomer vernachlässigt werden kann. 
Unter Beachtung des Ausschlusses von Transferreaktionen werden zunächst einige Unzulänglichkeiten bei der Auswertung nach der idealen Polymerisationskinetik dargestellt. In SP-PLP-Experimenten nimmt die Länge der wachsenden Radikale linear mit der Zeit zu. Werden nun für die Auswertung mit kettenlängenunabhängigem $k_{\mathrm{t}}$ (Gleichung 3.6) verschiedene Zeitintervalle nach Initiierung durch den Laser betrachtet, so ergibt sich jeweils ein mittlerer Terminierungsgeschwindigkeitskoeffizient $\left\langle k_{\mathrm{t}}\right\rangle$, der sich bei kürzerer Aufnahmezeit auf eine kleinere mittlere Kettenlänge bezieht. In Abbildung 8.3 ist $\left\langle k_{t}>\right.$ für Homopolymerisationen von MA und DMA bei $40{ }^{\circ} \mathrm{C} / 1000$ bar bis zu höheren Monomerumsätzen aufgetragen, wobei jeweils zwei verschiedene Zeitbereiche ausgewertet wurden.

Wenn der Terminierungsgeschwindigkeitskoeffizient nicht von der Kettenlänge abhängt, sollte $<k_{\mathrm{t}}>$ für beide Intervalle gleich sein. Wie aus der Abbildung hervorgeht, ist dies nicht der Fall.

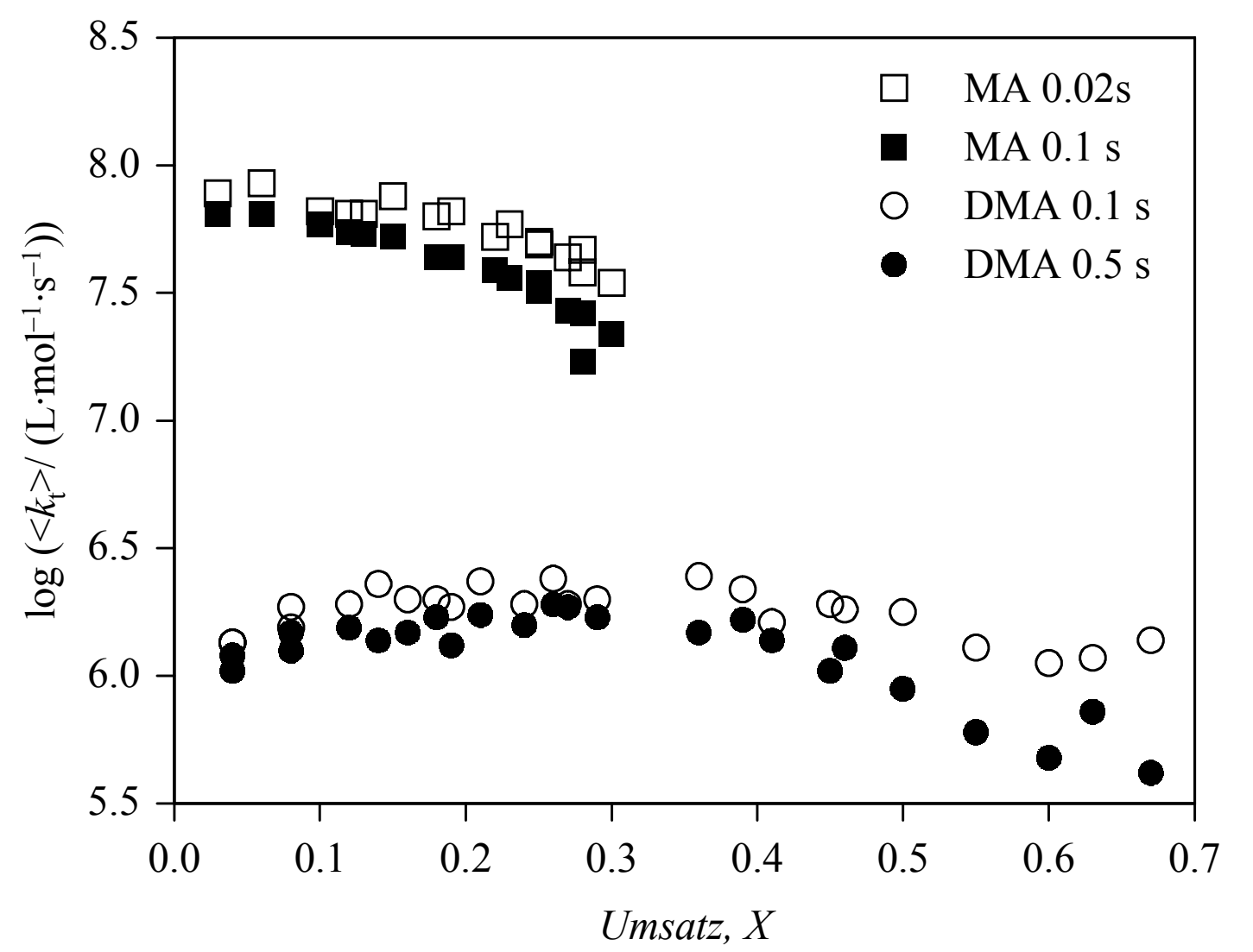

Abb. 8.3: Umsatzabhängigkeit von $\log \left\langle k_{t}>\right.$ für unterschiedliche Zeitbereiche nach dem Laserpuls bei $t=0$ für MA (Kreise) und DMA (Quadrate) bei $40{ }^{\circ} \mathrm{C} / 1000$ bar.

Bei beiden Monomeren sind die Werte für kleinere Zeitbereiche und demzufolge für kleinere Radikale (offene Symbole) größer als die Ergebnisse für Anpassungen derselben Signale über 
ein längeres Intervall. Die Unterschiede sind bei niedrigen und mittleren Umsätzen geringer als bei höheren Monomerumsätzen, wo die Datenpunkte klar voneinander getrennt sind. Die Daten aus Abbildung 8.3 lassen also auf eine Abnahme von $k_{\mathrm{t}}$ mit steigender Kettenlänge schließen. Ein ähnliches Bild ergibt sich für MMA und DA bei gleichen experimentellen Bedingungen, wie in Abbildung 8.4 gezeigt.

Auch für diese Monomere deuten die Ergebnisse auf eine Abnahme des Terminierungsgeschwindigkeitskoeffizienten mit steigender Kettenlänge hin. Im Fall von MMA ist die Differenz zwischen den entsprechenden $<k_{t}>$ Werten bei niedrigen Monomerumsätzen gering. Sie wird bei Umsätzen größer $20 \%$ erheblich.

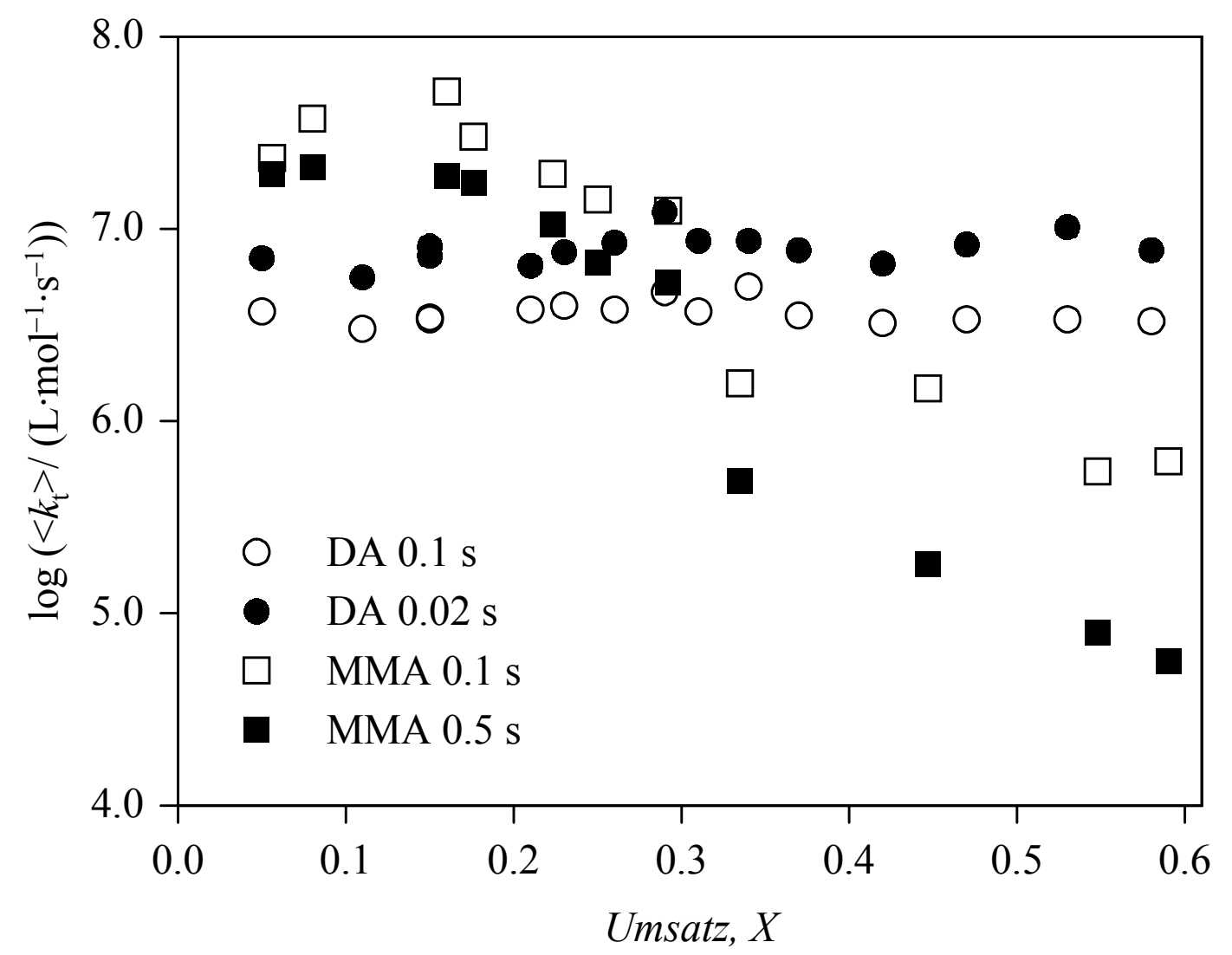

Abb. 8.4: Umsatzabhängigkeit von $\log \left\langle k_{t}>\right.$ für unterschiedliche Zeitbereiche nach dem Laserpuls bei $t=0$ für MMA bei (Kreise) und DA (Quadrate) bei $40{ }^{\circ} \mathrm{C} / 1000$ bar.

Für DA ergibt sich bei niedrigen Monomerumsätzen ein größerer Unterschied zwischen den $<k_{\mathrm{t}}>$-Werten für die betrachteten Intervalle als bei MA, DMA und MMA (Abbildungen 8.3 und 8.4). Allerdings tritt hier keine Zunahme des Abstandes bei höheren Monomerumsätzen auf. 
Bei den in den Kapiteln 6 und 7 untersuchten Polymerisationen wurde DMPA als Photoinitiator verwendet. Es wurde trotz der in Kapitel 6 diskutierten Nichtidealität dieser Substanz in den meisten Fällen eine vom Augenschein her recht gute Anpassung der Messsignale nach idealer Polymerisationskinetik (Gleichung 3.6) erreicht. Offensichtlich können sich bei mit DMPA-initiierten Polymerisationen der Einfluss des inaktiven Initiatorfragments und die Kettenlängenabhängigkeit von $k_{\mathrm{t}} \mathrm{zu}$ einem gewissen Grad ausgleichen. Durch die Abnahme von $k_{\mathrm{t}}$ mit steigender Kettenlänge ergibt sich ein höherer Umsatz pro Laserpuls, was durch die Inhibierung wieder ausgeglichen wird. Bei den in diesem Kapitel vorgestellten Untersuchungen ist durch die Verwendung von MMMP als Photoinitiator eine Störung oder auch Kompensation durch inhibierende Radikale auszuschließen. Die Abweichungen von einer idealen Polymerisationskinetik sind in den Messsignalen klar zu erkennen, was in der Abbildung 8.5 für ein bei $40{ }^{\circ} \mathrm{C} / 1000$ bar bei einer Homopolymerisation von DA aufgenommenes Messsignal demonstriert ist.

In der hier dargestellten relativen Monomerkonzentration ist als durchgezogene Linie ein Fit nach idealer Polymerisationskinetik gezeigt. Es ist offensichtlich, dass mit Gleichung 3.6 keine zufriedenstellende Anpassung an das Signal erreicht wird. Die Messkurve wird zunächst durch den Fit überschätzt. Im mittleren Bereich liegt die berechnete Kurve dann unter und am Ende wieder über dem gemessenen Signal. 


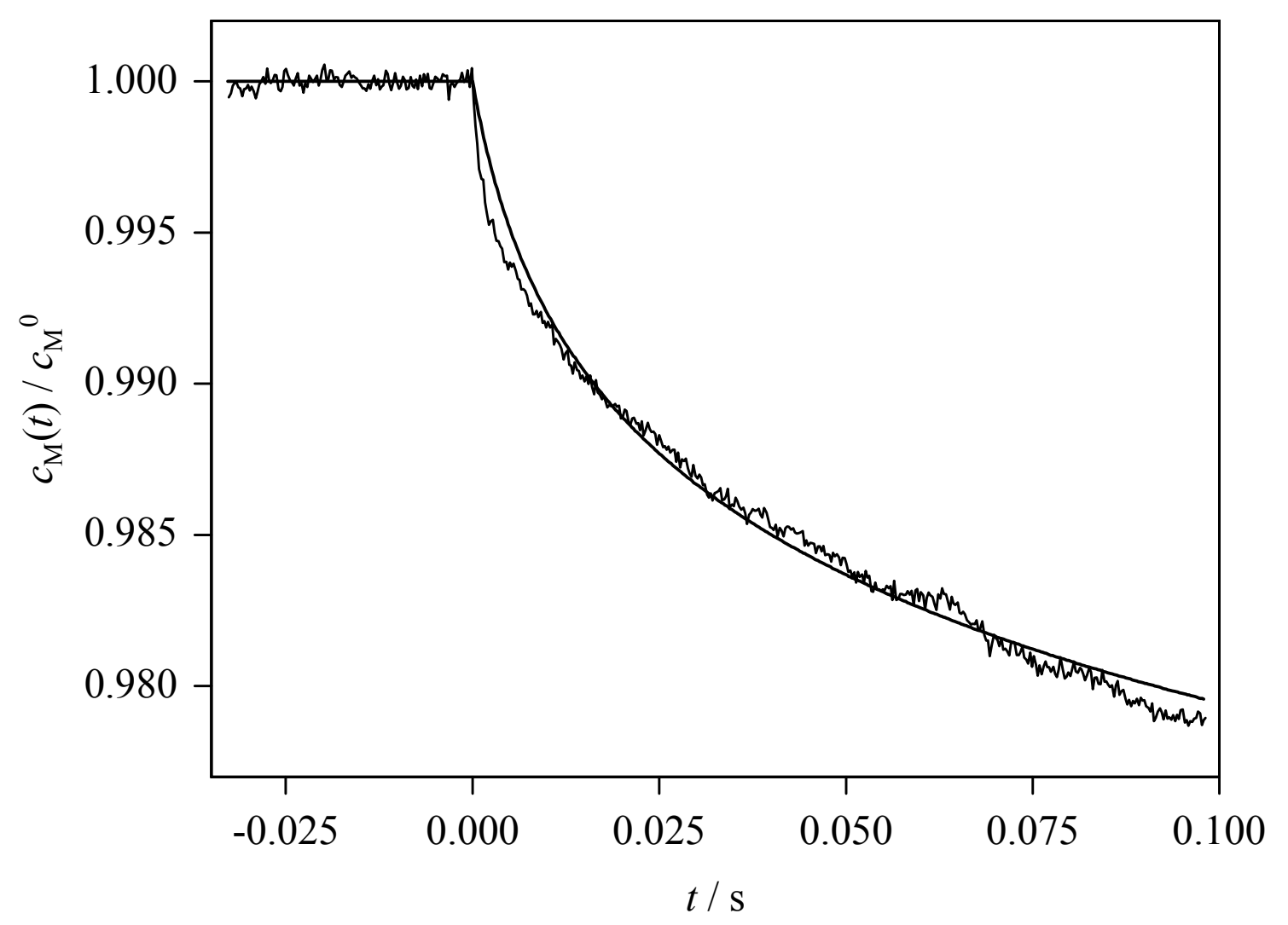

Abb. 8.5: Bei $40{ }^{\circ} \mathrm{C} / 1000$ bar und $11 \%$ Monomerumsatz aufgenommenes Messsignal für eine mit MMMP initiierte Homopolymerisation von DA. Die durchgezogene Linie kennzeichnet eine Anpassung nach idealer Polymerisationskinetik.

Zur besseren Beschreibung der Messsignale ist also die Berücksichtigung einer Kettenlängenabhängigkeit von $k_{\mathrm{t}}$ notwendig. Dazu sollen nun einige Konzepte vorgestellt werden.

Der weitreichendste Ansatz zur Auswertung von SP-PLP-Signalen wurde von de Kock et al. vorgeschlagen [8]. Mit Gleichung 8.1 (entspricht 3.3)

$$
\frac{\mathrm{d} c_{\mathrm{M}}}{\mathrm{d} t}=-k_{\mathrm{p}} \cdot c_{\mathrm{M}} \cdot c_{\mathrm{R}}
$$

lässt sich die Radikalkonzentration als Funktion der Zeit berechnen.

$$
c_{\mathrm{R}}(t)=-\frac{\mathrm{d} c_{\mathrm{M}}(t)}{\mathrm{d} t} \cdot \frac{1}{k_{\mathrm{p}} \cdot c_{\mathrm{M}}(t)}
$$

Dabei ist $\mathrm{d} c_{\mathrm{M}}(t) / \mathrm{d} t$ die erste Ableitung des Messsignals nach der Zeit. Aus Gleichung 8.3 (entspricht 3.4) 


$$
\frac{\mathrm{d} c_{\mathrm{R}}}{\mathrm{d} t}=-2 \cdot k_{\mathrm{t}} \cdot c_{\mathrm{R}}^{2}
$$

aufgelöst nach $k_{\mathrm{t}}$ ergibt sich:

$$
k_{\mathrm{t}}(t)=\frac{\mathrm{d} c_{\mathrm{R}}(t)}{\mathrm{d} t} \cdot \frac{1}{2 \cdot c_{\mathrm{R}}(t)^{2}}
$$

In 8.4 wird $\mathrm{d} c_{\mathrm{R}}(t) / \mathrm{d} t$ durch Ableitung von Gleichung 8.2 nach der Zeit erhalten. Somit ist zur Berechnung von $k_{\mathrm{t}}$ das zweimalige Differenzieren des Signals nach der Zeit notwendig. Wie bereits erwähnt, nimmt in einem SP-PLP-Experiment die Kettenlänge $i$ linear mit der Zeit zu:

$$
i(t)=k_{\mathrm{p}} \cdot c_{\mathrm{M}} \cdot t
$$

Es lässt sich also jedes $k_{\mathrm{t}}(t)$ direkt einem $k_{\mathrm{t}}^{\mathrm{i}, \mathrm{i}}$, dem Geschwindigkeitskoeffizienten der Terminierung von zwei Radikalen der Länge $i$, zuordnen. Dabei wird angenommen, dass zu jedem Zeitpunkt nur Radikale gleicher Länge terminieren. Diese Voraussetzung ist bis zu einem Auftreten einer breiteren Radikalverteilung durch Transfer zum Monomer oder Polymer in guter Näherung erfüllt. Da das Umsatz-Zeit-Signal zur Bestimmung von $k_{\mathrm{t}}$ zweimal nach der Zeit abgeleitet werden muss, sind die Anforderungen an die Signalqualität besonders hoch [9]. Eine solche Signalqualität wird mit der zur Verfügung stehenden Apparatur noch nicht erreicht, was in der Abbildung 8.6 demonstriert ist. Gezeigt sind die erste und zweiten Ableitung des Messsignals aus Abbildung 8.5 nach der Zeit.
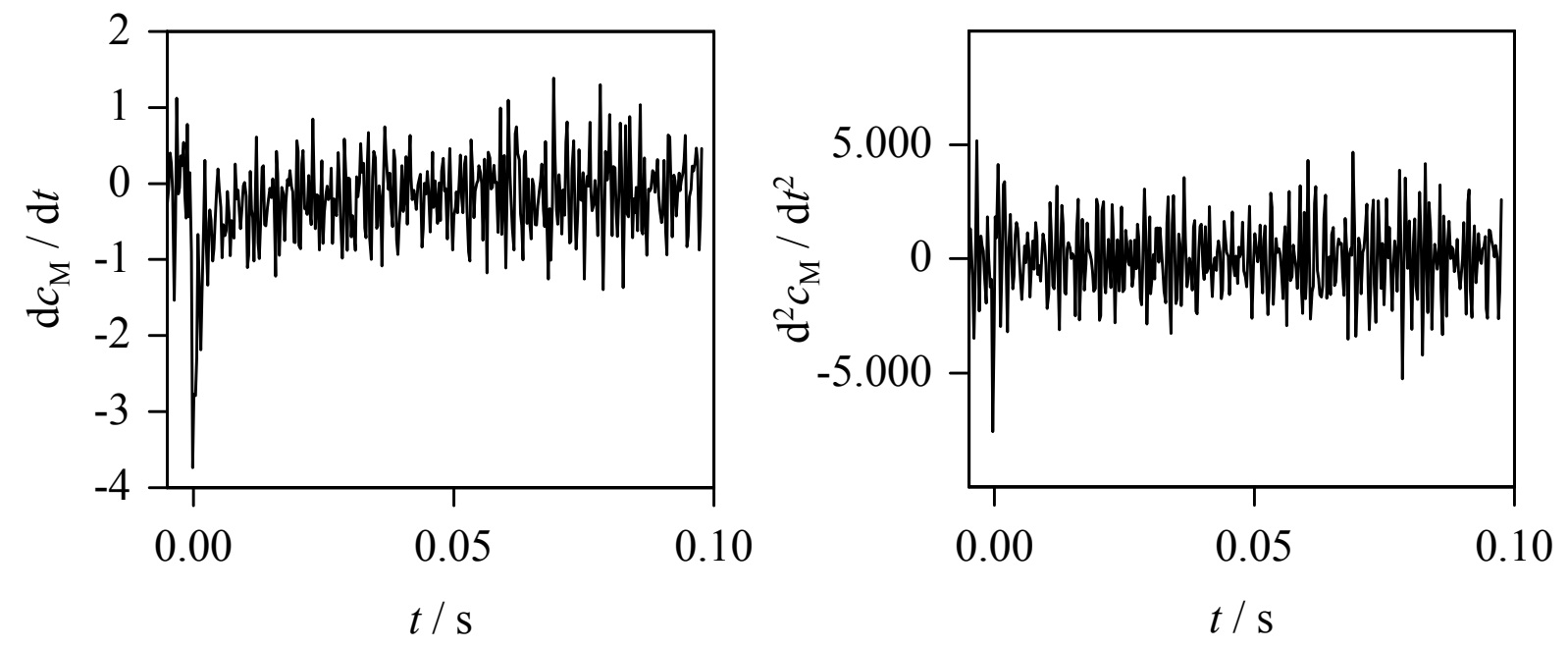

Abb. 8.6: Erste (linkes Bild) und zweite Ableitung (rechtes Bild) des Signals aus der Abbildung 8.5 nach der Zeit. 
Die große Streuung der Daten lässt offensichtlich eine Bestimmung der Kettenlängenabhängigkeit von $k_{\mathrm{t}}$ nach den Gleichungen 8.4 und 8.5 nicht zu.

Alternativ kann ein anderer Ansatz zur Untersuchung der Kettenlängenabhängigkeit von $k_{\mathrm{t}}$ verfolgt werden. Dabei wird ein Modell für ein kettenlängenabhängiges $k_{\mathrm{t}}$ vorgegeben und entsprechend an die Signale angepasst. In der Literatur werden zahlreiche Ansätze zur Beschreibung der kettenlängenabhängigen Terminierungsreaktion [z. B. 10, 11] diskutiert. Hier soll nur auf wenige Beispiele eingegangen werden. Eines der am häufigsten benutzten Modelle ist ein einfaches Potenzgesetz der Form

$$
k_{\mathrm{t}}^{i, i}=k_{\mathrm{t}}^{0} \cdot i^{-\alpha}
$$

wobei $k_{\mathrm{t}}^{0}$ der Terminierungsgeschwindigkeitskoeffizient für sehr kleine Radikale und $\alpha$ das Ausmaß der Kettenlängenabhängigkeit ist [12]. Dieser Ansatz wurde in der Literatur für diffusionskontrollierte $[13,14,15]$ Reaktionen diskutiert. Aber auch für chemisch kontrollierte Reaktionen makromolekularer Spezies fand Khokhlov [16] einen solchen Zusammenhang zwischen Kettenlänge und $k_{\mathrm{t}}$. Olaj et al. konnten durch einen Vergleich mit numerischen Verfahren zeigen, dass mit einem Potenzgesetz eine gute Beschreibung der Daten möglich ist [14].

Ein physikalisch sinnvolleres Modell basiert auf Überlegungen von de Gennes [17] und wurde von Buback et al. [18] ausführlich diskutiert. Es ist gültig für halb-verdünnte und konzentrierte Lösungen. Ein solches Szenario liegt für Polymerisationen bereits bei niedrigen Monomerumsätzen vor. Hier werden die Radikale in zwei Gruppen unterteilt:

a) Unterhalb einer kritischen Kettenlänge $i_{\mathrm{e}}$, bei SP-PLP verknüpft mit einer kritischen Zeit $t_{\mathrm{e}}$, verhalten sich die Radikale ideal. Es tritt keine Abhängigkeit von $k_{\mathrm{t}}$ von der Kettenlänge auf.

b) Überschreiten die Radikale die Kettenlänge $i_{\mathrm{e}}$, bzw. wachsen die Ketten länger als $t_{\mathrm{e}}$, so bilden sich Überlappungen und Verwicklungen (engl. „entanglements“) der wachsenden Ketten untereinander und mit totem Polymer. Dann nimmt $k_{\mathrm{t}}$ exponentiell mit der Kettenlänge ab, wobei ein Exponent von 3/2 vorhergesagt wird.

Es ergibt sich also folgender Ausdruck für $k_{\mathrm{t}}$ :

$$
k_{\mathrm{t}}(t)=k_{\mathrm{t}}^{0} \cdot \omega(t), \quad \omega(t)=\left\{\begin{array}{l}
1, t<t_{\mathrm{e}} \\
\left(t_{\mathrm{e}} / t\right)^{3 / 2}, t>t_{\mathrm{e}}
\end{array}\right.
$$


Dieses Modell lässt sich noch weiter verfeinern, etwa durch Einführen einer Kettenlängenabhängigkeit auch für Radikale kleiner $i_{\mathrm{e}}$, dann allerdings mit einem kleineren Exponenten. Ebenso ist auch eine Vereinfachung dahingehend möglich, dass für Ketten größer $i_{\mathrm{e}}$ ein ebenfalls konstantes, aber kleineres $k_{\mathrm{t}}$ angenommen wird. Dann entsteht ein Ausdruck in Form einer Stufenfunktion. Der mit Gleichung 8.7 beschriebene Fall (3) ist zusammen mit dem Potenzgesetz aus Gleichung 8.6 (2) und kettenlängenunabhängigem $k_{\mathrm{t}}(\mathbf{1})$ in Abbildung 8.7 veranschaulicht.

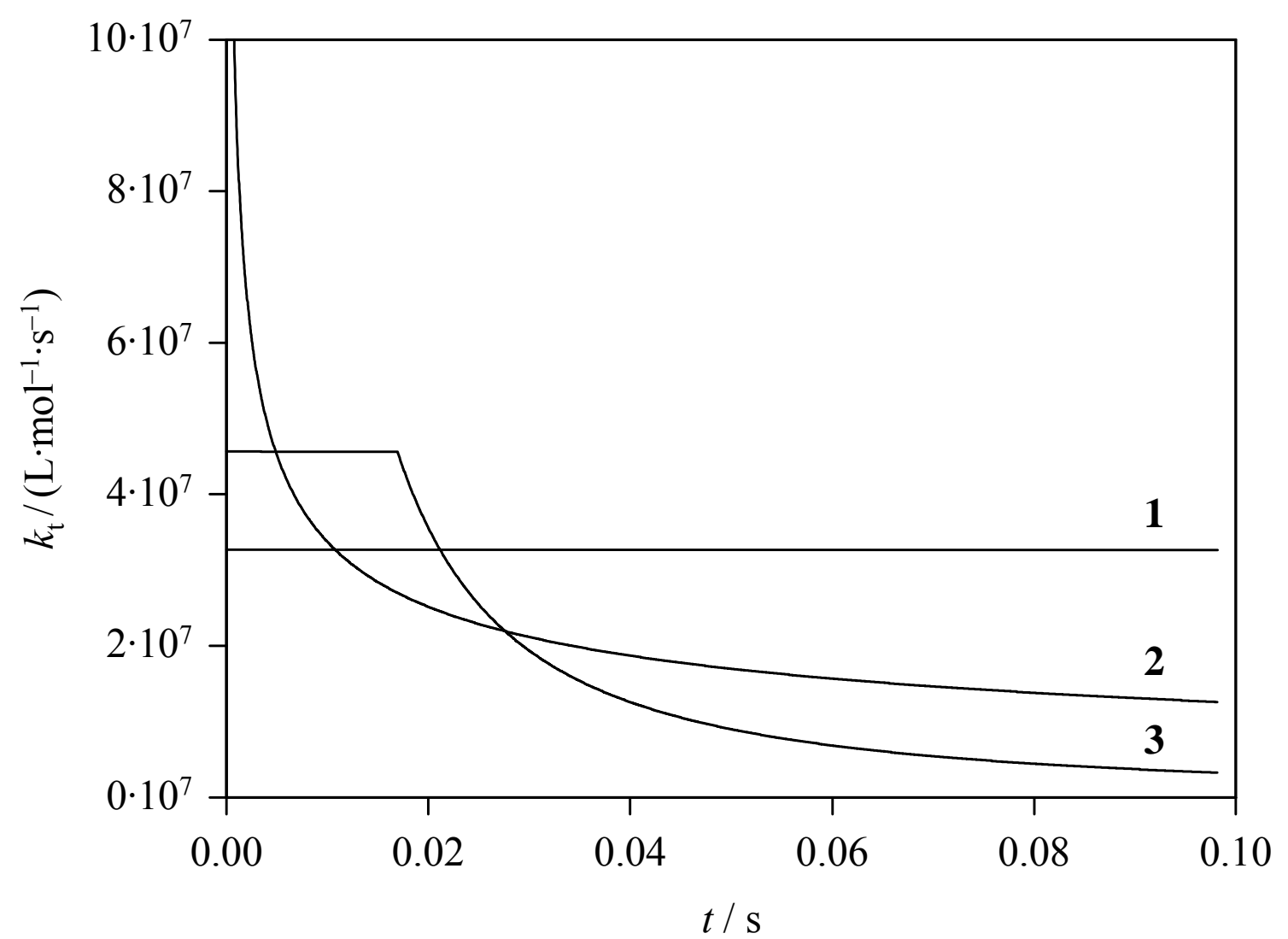

Abb. 8.7: Schematische Darstellung von Modellen für $k_{\mathrm{t}}$. Die ideale Kinetik ist mit 1 gekennzeichnet, $\mathbf{2}$ zeigt ein Potenzgesetz nach Gleichung 8.6 und $\mathbf{3}$ den Ansatz aus Gleichung 8.7.

Bevor nun die Bestimmung der Kettenlängenabhängigkeit von $k_{\mathrm{t}}$ mit Hilfe eines der beschriebenen Modelle vorgenommen wird, muss geklärt werden, ob anhand der Signale entschieden werden kann, welcher Ansatz die Realität am besten beschreibt. Dazu werden an das Signal für die DA-Homopolymerisation aus Abbildung 8.5 (bei dem die Anpassung mit Modell 1 dargestellt ist) die in 8.7 gezeigten Modelle 2 und $\mathbf{3}$ angepasst. Die entsprechenden Fits sind in Abbildung 8.8 gezeigt. 


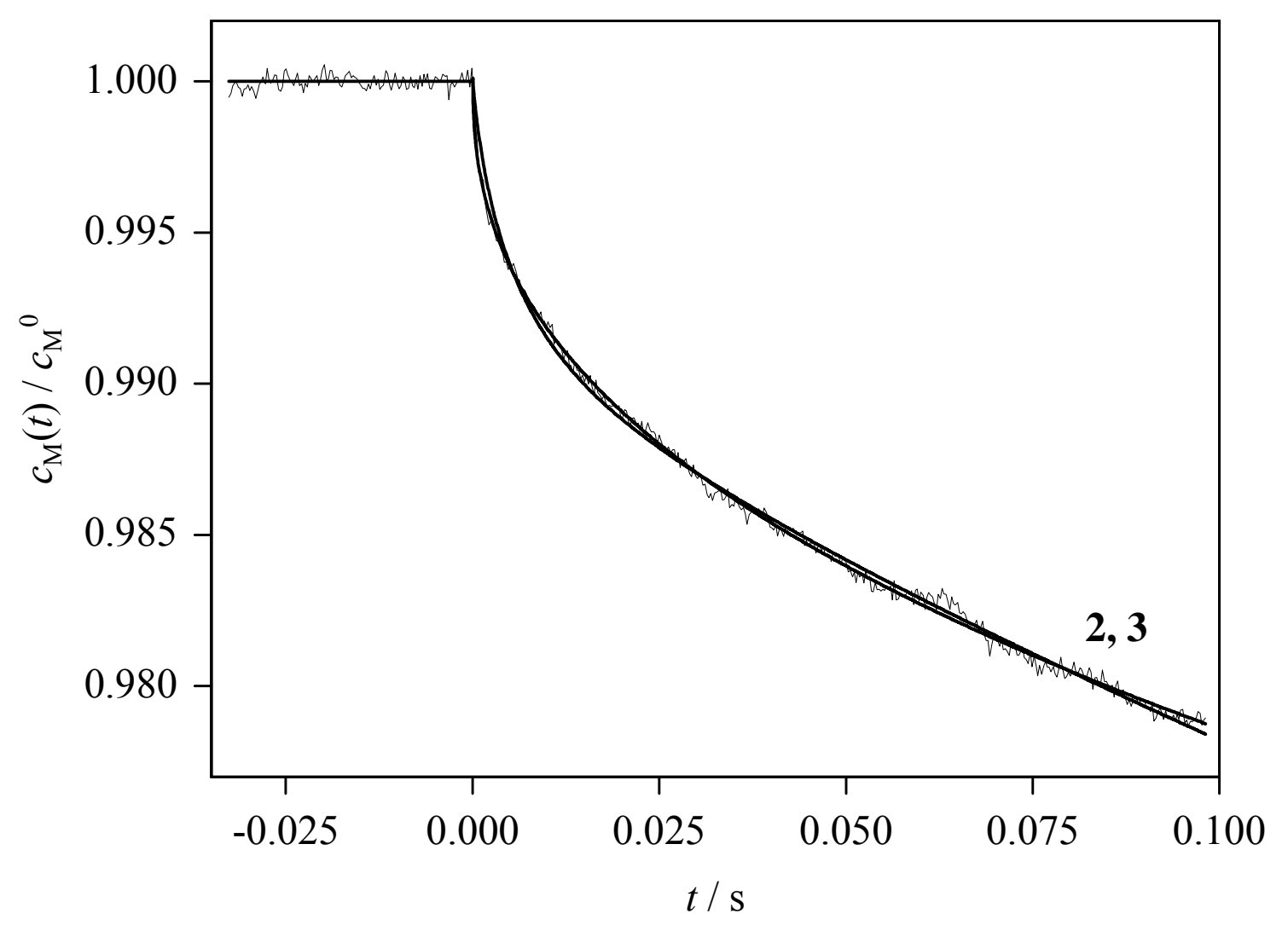

Abb. 8.8: Anpassung der in Abbildung 8.8 veranschaulichten Modelle 2 und $\mathbf{3}$ für $k_{\mathrm{t}}$ an das in 8.5 gezeigte Messsignal für eine mit MMMP initiierte Homopolymerisation von DA bei $40{ }^{\circ} \mathrm{C} / 1000$ bar.

Aus der Auftragung geht hervor, dass beide Modelle zur Kettenlängenabhängigkeit von $k_{\mathrm{t}}$ eine sehr gute Anpassung an das Messsignal ermöglichen. Allerdings ist bei der vorliegenden Signalqualität unklar, welcher Ansatz zu bevorzugen ist. Für eine solche Entscheidung wäre ein deutlich günstigeres Signal-Rausch-Verhältnis erforderlich. Für SP-PLP-Experimente ist DA entsprechend den Ausführungen in Kapitel 6.1.1 das am besten geeignete Monomer aus den Familien der Acrylate und Methacrylate, so dass sich für andere Monomere eine schlechtere Signalqualität ergibt und eine Modellunterscheidung erst recht unmöglich ist. Als Fazit resultiert aus diesen Betrachtungen, dass aus der Qualität der Anpassung von SP-PLPSignalen keine Aussage über die Güte der Modelle zur Kettenlängenabhängigkeit von $k_{\mathrm{t}}$ möglich ist. Allenfalls können die bei den Fits resultierenden Parameter und die daraus folgenden Aussagen Aufschlüsse über die Anwendbarkeit der hinter den Modellen stehenden mechanistischen Vorstellungen liefern.

Im Rahmen dieser Arbeit wird wegen der weiten Verbreitung und damit verbundenen Vergleichbarkeit der Ergebnisse das als Gleichung 8.6 vorgestellte Potenzgesetz verwendet. 
Im Vergleich zu dem mit Gleichung 8.7 beschriebenen Modell wird bei dem Potenzgesetz das Ausmaß der Kettenlängenabhängigkeit mit einem „effektiven“ Exponenten $\alpha$ beschrieben. Dieser Exponent gilt dann für die gesamte Signalaufnahmezeit und damit auch für alle betrachteten Kettenlängen. Mit einer durch 8.6 beschriebenen Kettenlängenabhängigkeit von $k_{\mathrm{t}}$ ergibt sich für die Abnahme der relativen Monomerkonzentration nach einem Laserpuls bei $t=0$ der folgende Ausdruck [18]:

$$
\frac{c_{\mathrm{M}}(t)}{c_{\mathrm{M}}^{0}}=1-\frac{b}{c} \cdot \int_{0}^{t}\left(b \int_{0}^{t^{\prime}}\left(t_{\mathrm{p}} / t^{\prime \prime}\right)^{\alpha} \mathrm{d} t^{\prime \prime}+1\right)^{-1} \mathrm{~d} t^{\prime}
$$

In dieser Gleichung ist $t_{\mathrm{p}}=\left(k_{\mathrm{p}} \cdot c_{\mathrm{M}}^{0}\right)^{-1}$ die für einen Wachstumsschritt benötigte Zeit (und für jeden folgenden Schritt bei diesem SP-PLP-Experiment mit $c_{\mathrm{M}} \cong$ konstant, da $\Delta c_{\mathrm{M}}$ pro Puls klein ist), $b \equiv 2 \cdot c_{\mathrm{R}}^{0} \cdot k_{\mathrm{t}}^{0}$ und $c \equiv 2 \cdot k_{\mathrm{t}}^{0} / k_{\mathrm{p}}$. Bei Gleichung 8.8 wird angenommen, dass Transferprozesse ignoriert werden können. Diese Näherung ist gerechtfertigt für den Anfangsbereich eines SP-PLP-Signals bei Zeiten $t<t_{\mathrm{tr}}$, wobei $t_{\mathrm{tr}}=\left(k_{\mathrm{tr}} \cdot c_{\mathrm{M}}\right)^{-1}$ den Zeitraum darstellt, nach dem Kettenübertragung bedeutsam wird.

Wie bereits erwähnt, wird hier davon ausgegangen, dass Signale aus Messungen mit Acrylaten bis $0.1 \mathrm{~s}$ und Kurven aus Untersuchungen mit Methacrylaten bis $0.5 \mathrm{~s}$ unter Vernächlässigung von Transferreaktionen zum Monomer oder Polymer ausgewertet werden können.

Mit Gleichung 8.8 lassen sich auch Messsignale von für Untersuchungen mit der SP-PLPTechnik weniger gut geeigneten Monomeren wie MMA beschreiben, wie in Abbildung 8.9 gezeigt ist. Allerdings wurde dieses Experiment zur Verbesserung des Signal-RauschVerhältnisses bei einem höheren Druck von 2000 bar durchgeführt. Über den gesamten Analysezeitraum zeigt der Fit keine signifikante Abweichung von der gemessenen relativen Monomerkonzentration. 


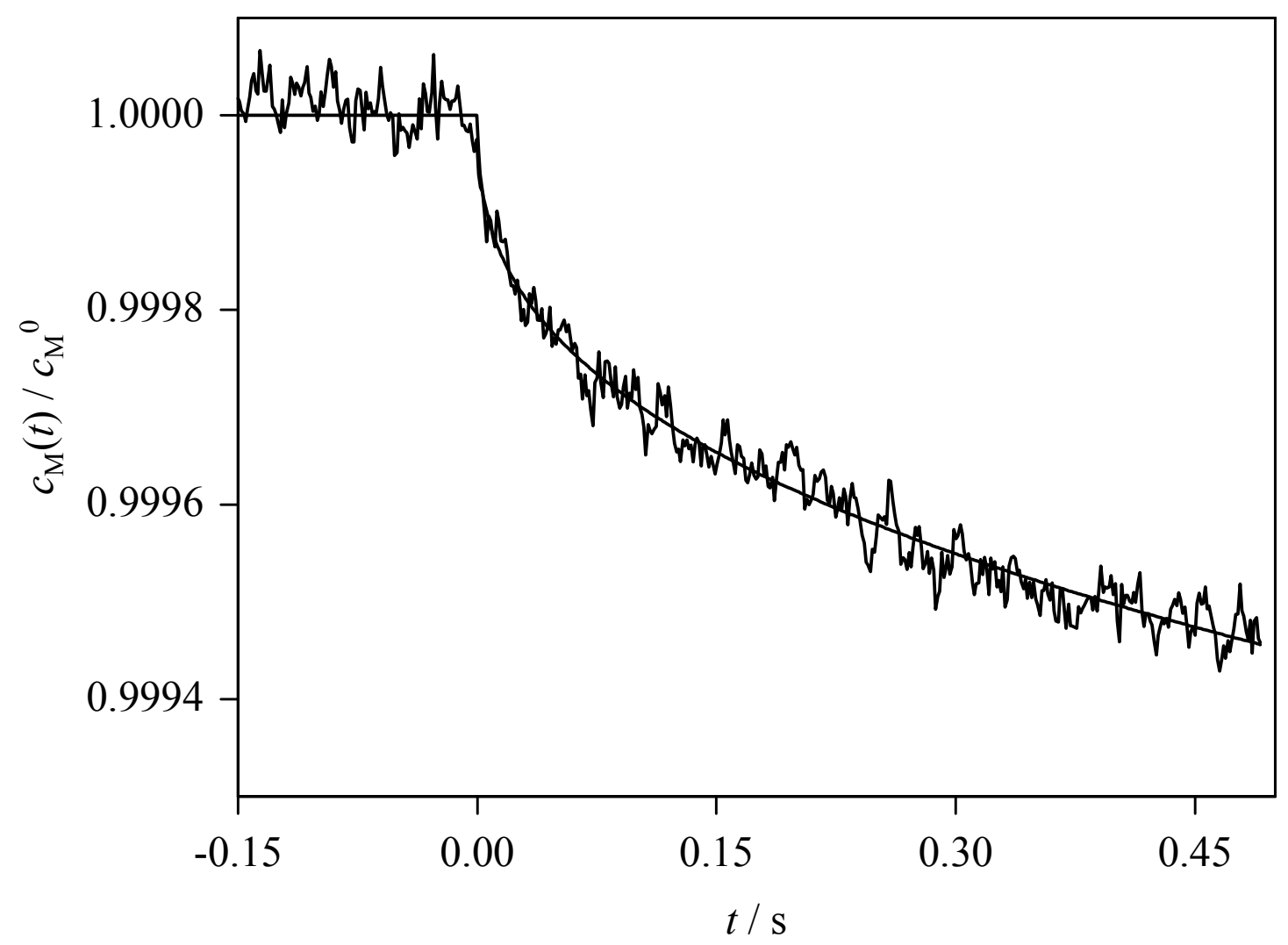

Abb. 8.9: Bei $40{ }^{\circ} \mathrm{C} / 2000$ bar und $19 \%$ Monomerumsatz aufgenommenes Messsignal für eine mit MMMP initiierte Homopolymerisation von MMA. Die durchgezogene Linie kennzeichnet eine Anpassung mit Gleichung 8.8.

Bei einer Anpassung der Gleichung 8.8 an die experimentell bestimmten relativen Monomerkonzentrationen werden die Parameter $b, c$ und $\alpha$ erhalten, woraus sich schließlich auch der Terminierungsgeschwindigkeitskoeffizient für sehr kleine Radikale, $k_{\mathrm{t}}^{0}$, ermitteln lässt. In diesem Kapitel sollen die in Gleichung 8.6 vorgestellten Parameter $k_{\mathrm{t}}{ }^{0}$ und $\alpha$ für eine Reihe von Monomeren bestimmt und diskutiert werden. Mit der hier verwendeten Methodik ergeben sich diese Größen paarweise für jedes einzelne Messsignal bis zu hohen Monomerumsätzen. Eine entsprechende Auswertung ist für eine Serie von Messkurven für MMA-Homopolymerisationen bei $40{ }^{\circ} \mathrm{C} / 2000$ bar (analog zu dem Signal aus 8.8) in der Abbildung 8.10 dargestellt, wobei die Kreise $\alpha$ und die Quadrate $k_{\mathrm{t}}^{0}$ symbolisieren. Bei diesen Bedingungen gelingen SP-PLP-Experimente bis zu Monomerumsätzen von etwa $55 \%$ in homogener Phase. 


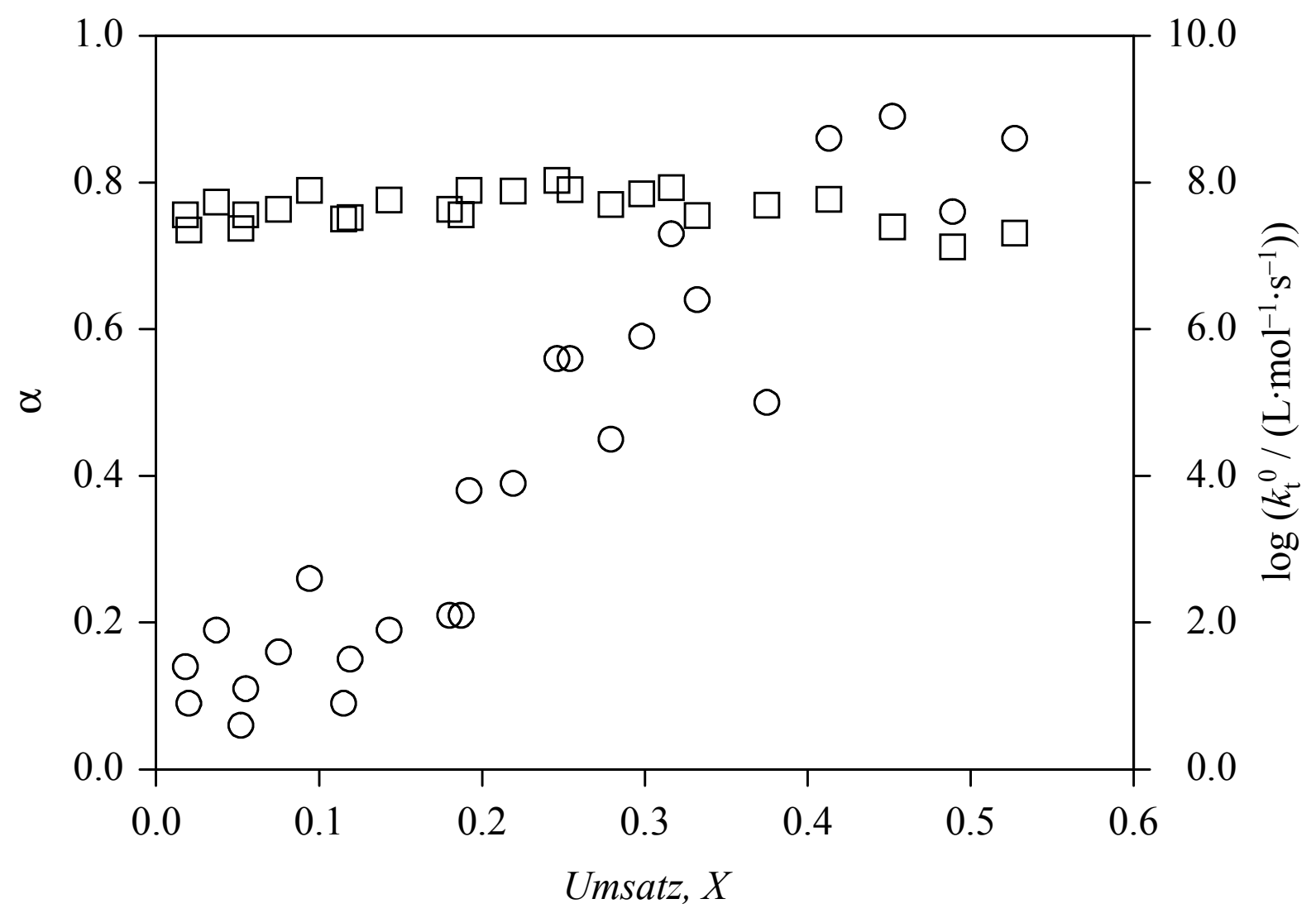

Abb. 8.10: Exponent $\alpha$ (Kreise, Ordinate links) und $\log k_{\mathrm{t}}^{0}$ (Quadrate, Ordinate rechts) für MMA-Homopolymerisationen bei $40{ }^{\circ} \mathrm{C} / 2000$ bar aus der Anpassung von Gleichung 8.8 .

Die Ergebnisse für $\alpha$ weisen in dieser Auftragung eine größere Streuung auf als die Werte für $k_{\mathrm{t}}^{0}$, was aber im Wesentlichen durch die logarithmische Skala beim Terminierungsgeschwindigkeitskoeffizienten hervorgerufen wird.

Der Parameter $k_{\mathrm{t}}^{0}$ ist nahezu umsatzunabhängig, erst bei Umsätzen über $40 \%$ ist ein leichter Abfall zu beobachten. Für $\alpha$ ergibt sich bis etwa $15 \%$ Umsatz innerhalb der Streuung ein konstanter Wert, dann folgt ein starker Anstieg mit dem Umsatz.

Bei genauer Betrachtung der Abbildung zeigt sich jedoch, dass die Parameter $\alpha$ und $k_{\mathrm{t}}^{0}$ miteinander korreliert sind. Im niedrigen Umsatzbereich bis etwa $15 \%$ ergibt sich für einen höheren Wert von $\alpha$ auch ein höheres Ergebnis für $k_{\mathrm{t}}^{0}$. Mit Eintritt in den Geleffekt bei einem Monomerumsatz von etwa $20 \%$ verliert sich diese Korrelation, die Steigerung bei $\alpha$ ist nicht mehr mit einer entsprechenden Vergrößerung bei $k_{\mathrm{t}}^{0}$ verbunden. Die Abhängigkeit der beiden Parameter zu Beginn der Polymerisation ist in der Abbildung 8.11 veranschaulicht. Aus dieser Auftragung ist auch ersichtlich, dass die Unsicherheit für beide Werte etwa in der gleichen Größenordung liegt. 


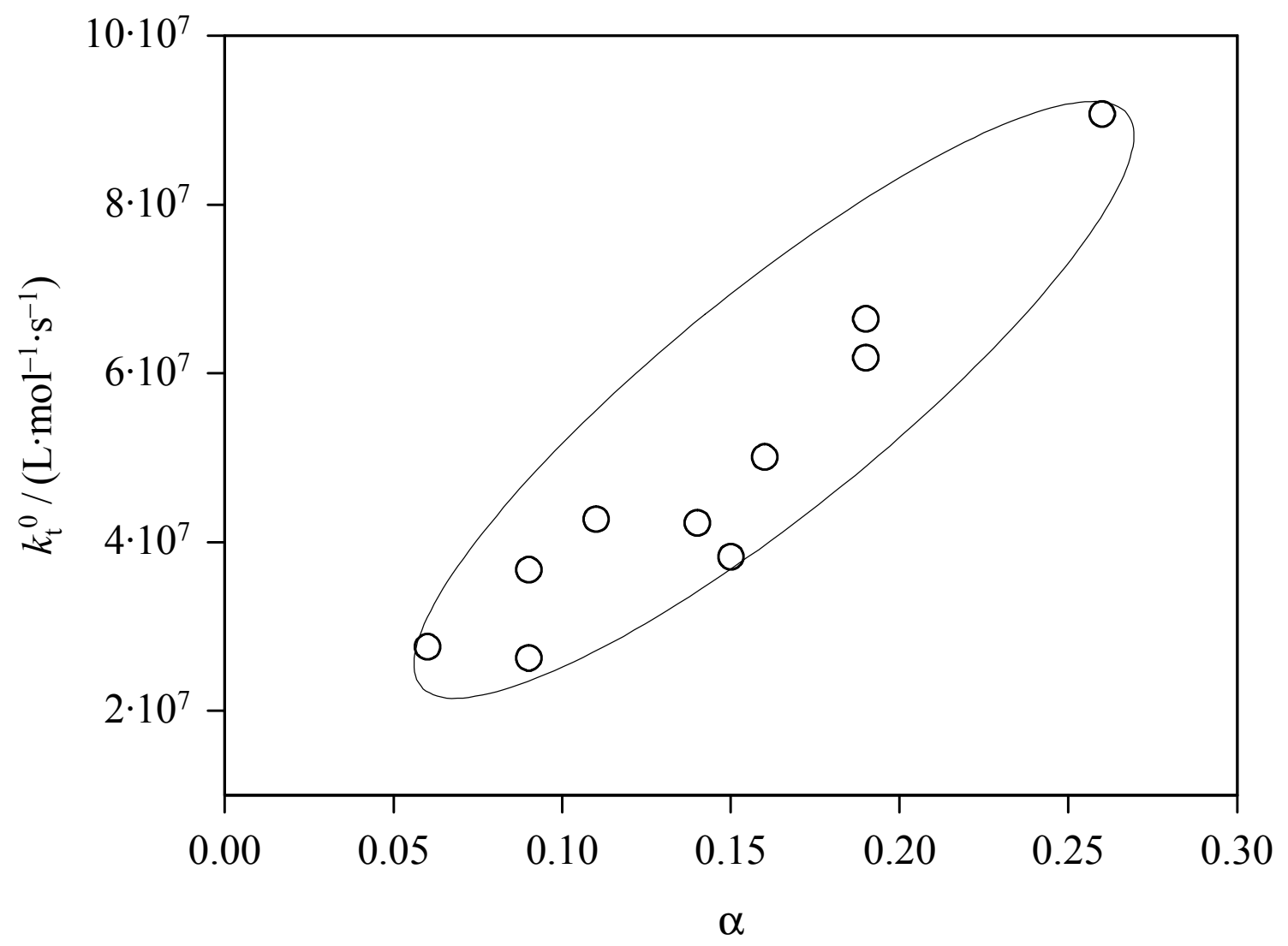

Abb. 8.11: Korrelation der Parameter $\alpha$ und $k_{\mathrm{t}}^{0}$ bei Monomerumsätzen kleiner $15 \%$ für die MMA-Homopolymerisation bei $40{ }^{\circ} \mathrm{C} / 2000$ bar.

Angesichts dieser Abhängigkeit der Parameter voneinander erscheint es gerechtfertigt, die Fitprozedur nach Gleichung 8.8 durch ein unabhängiges Verfahren zu überprüfen. Dazu wird nun eine andere Möglichkeit der Auswertung abgeleitet.

Es sei $c_{\mathrm{M}}$ die Monomerkonzentration und $c_{\mathrm{R}}$ die Radikalkonzentration. Zur Vereinfachung der Herleitung sollen die Einheiten so gewählt sein, dass für die Wachstumsgeschwindigkeit $v_{\mathrm{p}}$ gilt: $v_{\mathrm{p}}=k_{\mathrm{p}} \cdot c_{\mathrm{M}} \equiv 1$. Damit ergeben sich folgende Vereinfachungen:

$$
\begin{aligned}
& \frac{\mathrm{d} c_{\mathrm{M}}}{\mathrm{d} t}=-k_{\mathrm{p}} \cdot c_{\mathrm{M}} \cdot c_{\mathrm{R}}=-v_{\mathrm{p}} \cdot c_{\mathrm{R}}=-c_{\mathrm{R}} \\
& i(t)=k_{\mathrm{p}} \cdot c_{\mathrm{M}} \cdot t=v_{\mathrm{p}} \cdot t=t
\end{aligned}
$$

Es gelte außerdem das mit Gleichung 8.6 bezeichnete Potenzgesetz für $k_{\mathrm{t}}$. Gleichung 8.3 lässt sich dann integrieren und umformen $\mathrm{zu}$ : 


$$
\frac{c_{\mathrm{R}}(t)}{c_{\mathrm{R}}^{0}}=\frac{1}{1+\int_{0}^{t} c_{\mathrm{R}}^{0} \cdot k_{\mathrm{t}}(t) \mathrm{d} t} \approx \frac{1}{1+\left(t / \tau_{0}\right)^{1-\alpha}} \quad(\alpha<1)
$$

mit: $\quad \tau_{0} \equiv\left(\frac{c_{\mathrm{R}}^{0} \cdot k_{\mathrm{t}}^{0}}{1-\alpha}\right)^{\frac{1}{\alpha-1}} \quad$ bei $v_{\mathrm{p}} \equiv 1$

$\tau_{0}$ entspricht für $\alpha<<1$ der Zeit, die für die Terminierung von zwei kleinen Radikalen benötigt wird. Gleichung 8.11 lässt sich in eine Reihe entwickeln. Dabei werden zwei Fälle unterschieden:

$$
\begin{aligned}
& \frac{c_{\mathrm{R}}(t)}{c_{\mathrm{R}}^{0}} \approx 1-\left(\frac{t}{\tau_{0}}\right)^{1-\alpha} \approx 1, \quad t<<\tau_{0} \\
& \frac{c_{\mathrm{R}}(t)}{c_{\mathrm{R}}^{0}} \approx\left(\frac{\tau_{0}}{t}\right)^{1-\alpha}, \quad t>>\tau_{0}
\end{aligned}
$$

Außerdem ist folgende Umformung von Gleichung 8.9 möglich:

$$
\frac{c_{\mathrm{M}}(t)}{c_{\mathrm{M}}^{0}}=1-\frac{1}{c_{\mathrm{M}}^{0}} \int_{0}^{t} c_{\mathrm{R}}(t) \mathrm{d} t
$$

oder

$$
\frac{c_{\mathrm{M}}^{0}-c_{\mathrm{M}}(t)}{c_{\mathrm{M}}^{0}} \equiv \phi(t)=\frac{1}{c_{\mathrm{M}}^{0}} \int_{0}^{t} c_{\mathrm{R}}(t) \mathrm{d} t
$$

Hier wird als $\phi(t)$ der Monomerumsatz nach einem Laserpuls definiert. Einsetzen von 8.13 in 8.16 und Integrieren von 8.16 ergibt:

$$
\frac{c_{\mathrm{M}}^{0} \phi(t)}{c_{\mathrm{R}}^{0}}=\int_{0}^{t} c_{\mathrm{R}} / c_{\mathrm{R}}^{0} \mathrm{~d} t \approx t, \quad t<<\tau_{0}
$$

Entsprechend folgt mit 8.13 und 8.15:

$$
\frac{c_{\mathrm{M}}^{0} \phi(t)}{c_{\mathrm{R}}^{0}} \approx \tau_{0}\left(\frac{t}{\tau_{0}}\right)^{\alpha}, \quad t>>\tau_{0}
$$

Schließlich erhält man durch Logarithmieren von 8.17 und 8.18: 


$$
\begin{gathered}
\log \frac{c_{\mathrm{M}}^{0}}{c_{\mathrm{R}}^{0}}+\log \phi(t)=\log t, \quad t<<\tau_{0} \\
\log \frac{c_{\mathrm{M}}^{0}}{c_{\mathrm{R}}^{0}}+\log \phi(t)=\log \tau_{0}-\alpha \log \tau_{0}+\alpha \log t, \quad t>>\tau_{0}
\end{gathered}
$$

Aus den Gleichungen 8.19 und 8.20 ist ersichtlich, dass für eine Kettenlängenabhängigkeit nach dem mit 8.6 definierten Potenzgesetz eine Auftragung von $\log \phi(t)$ gegen $\log t$ Folgendes zeigen sollte: Die Daten nähern sich für Zeiten $t>>\tau_{0}$ asymptotisch einer Geraden mit der Steigung $\alpha$ und für Zeiten $t<<\tau_{0}$ einer Geraden mit der Steigung eins an. Eine solche Auftragung ist in der Abbildung 8.12 für eine MA-Homopolymerisation bei $40{ }^{\circ} \mathrm{C} / 1000 \mathrm{bar}$ und $12 \%$ Monomerumsatz mit dem dazugehörigen Messsignal gezeigt.

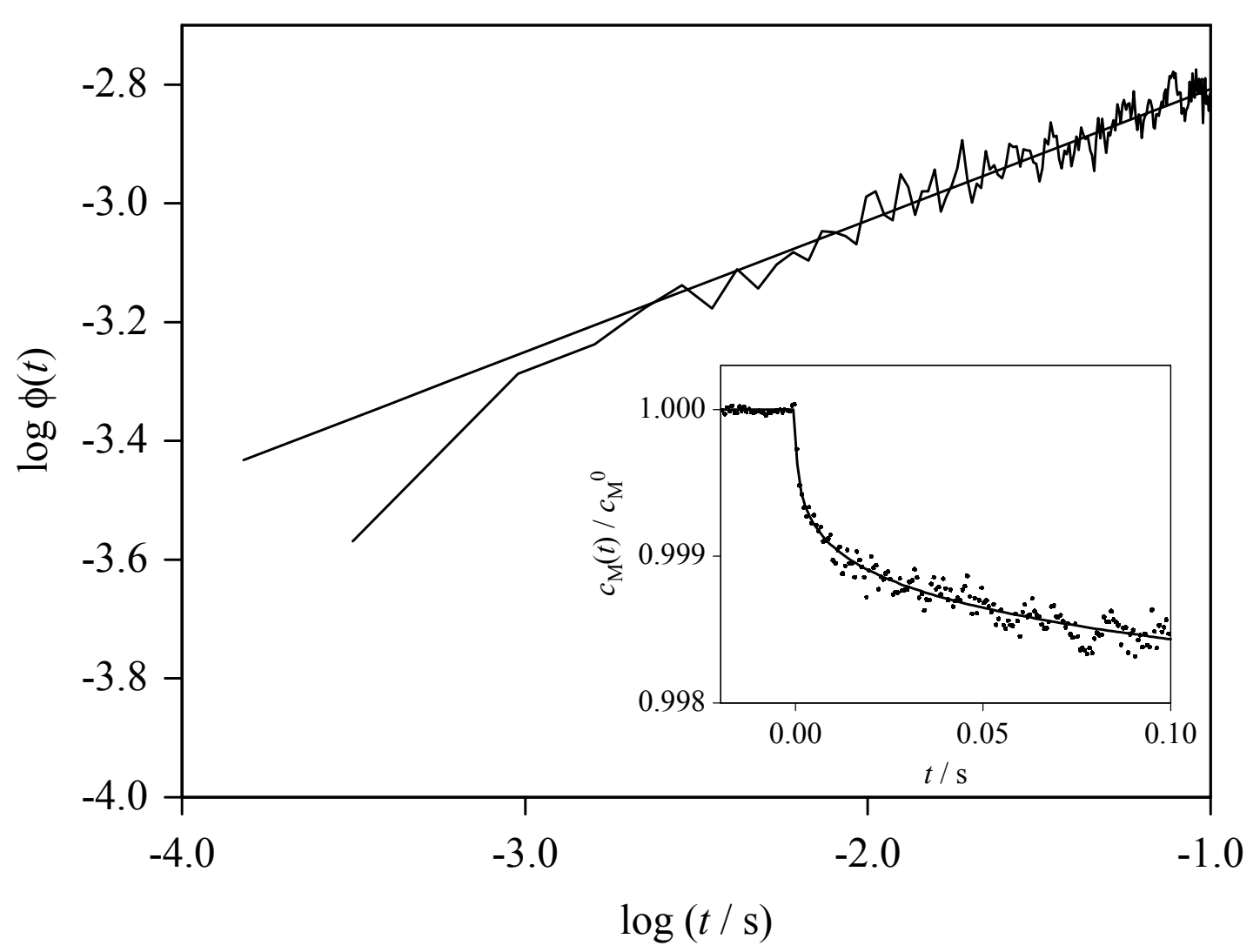

Abb. 8.12: Auftragung von $\log \phi(t)$ gegen $\log (t / \mathrm{s})$ für ein Messsignal aufgenommen bei einer MA-Homopolymerisation bei $40{ }^{\circ} \mathrm{C} / 1000$ bar und $12 \%$ Monomerumsatz. Die verkleinerte Darstellung zeigt dieses Signal und eine Anpassung nach Gleichung 8.8 . 
Zur Veranschaulichung wurde MA wegen der besseren Signalqualität ausgewählt. Für das vorher diskutierte MMA ergibt sich aber ein ähnliches Bild. Es sollte noch erwähnt werden, dass die hier vorgestellte Methode zur Auswertung von SP-PLP Signalen nicht gültig ist, wenn $\alpha$ nahe oder größer eins ist.

Wie in der Abbildung zu erkennen, wird eine asymptotische Annäherung von log $\phi(t)$ an eine Gerade bei $\log t>>-2.5$ bzw. Zeiten $t>>10^{-3} \mathrm{~s}$ erreicht. Die Steigung der Geraden entspricht dem Exponenten $\alpha$, der in diesem Fall $0.21 \pm 0.05$ ist. Dieses Ergebnis ist in guter Übereinstimmung mit dem Wert von $\alpha=0.15$, der aus einem direkten Fit von Gleichung 8.8 an das Messsignal resultiert (auch dargestellt in Abbildung 8.12). Es ist noch zu bedenken, dass die Bestimmung des Bereichs, für den die Anpassung mit einer Geraden durchgeführt wird, einen zusätzlichen Fehler ergibt.

Auch für kurze Zeiten nach dem Laserpuls ergibt sich ein linearer Zusammenhang von $\log \phi(t)$ und $\log t$ mit einer Steigung etwas kleiner eins, jedoch ist dies wegen der geringen Anzahl von Datenpunkten für $t<10^{-3} \mathrm{~s}$ mit einer größeren Unsicherheit behaftet.

Insgesamt bestärkt also die hier vorgestellte alternative Auswertung von Messsignalen die Richtigkeit der mit der direkten Anpassung von Gleichung 8.8 verbundenen Prozedur. Im nächsten Abschnitt werden nun die Ergebnisse für die Auswertung der Messkurven nach 8.8 für verschiedene Monomere vorgestellt und diskutiert.

\subsection{Ergebnisse und Diskussion}

\subsubsection{Terminierungsgeschwindigkeitskoeffizient $k_{\mathrm{t}}{ }^{0}$}

In Abbildung 8.10 wurde gezeigt, dass sich bei der Auswertung nach Gleichung 8.8 die Parameter $k_{\mathrm{t}}^{0}$ und $\alpha$ für jedes Messsignal ergeben. Zunächst sollen einige Ergebnisse für $k_{\mathrm{t}}^{0}$ betrachtet werden. An dieser Stelle muss noch erwähnt werden, dass in Gleichung 8.8 das mit 8.6 bezeichnete Potenzgesetz für die gesamte Signalaufnahmezeit und damit für alle Kettenlängen verwendet wird. Dann ist $k_{\mathrm{t}}^{0}$ formal der Terminierungsgeschwindigkeitskoeffizient für Radikale der Kettenlänge eins. Vana et al. [19] haben jedoch bei Experimenten mit Styrol gefunden, dass sich für Radikale mit Kettenlängen kleiner 10 eine wesentlich stärkere Kettenlängenabhängigkeit von $k_{\mathrm{t}}$ ergibt und Gleichung 8.6 nur für Kettenlängen zwischen 10 und 100 angewendet. Dann jedoch ist $k_{\mathrm{t}}^{0}$ nur ein Fitparameter und entspricht lediglich der Extrapolation auf die Kettenlänge eins. Vana et al. [19] konnten experimentell direkt das $k_{\mathrm{t}}$ für Radikale der Kettenlänge eins bestimmen. Dieser mit $k_{\mathrm{t}}^{1,1}$ bezeichnete 
Koeffizient ist in guter Übereinstimmung mit Literaturdaten für die Dimerisierung von kleinen Radikalen [20]. Wegen der gefundenen stärkeren Kettenlängenabhängigkeit bei sehr kleinen Radikalen (Kettenlängen zwischen eins und zehn) ist $k_{\mathrm{t}}^{1,1}$ fast eine Größenordnung höher als das extrapolierte $k_{\mathrm{t}}^{0}$. Aus den in dieser Arbeit untersuchten Messsignalen ist jedoch der Bereich solch kleiner Kettenlängen nicht zugänglich. Im Folgenden wird deshalb nur $k_{t}^{0}$ betrachtet und so der Terminierungsgeschwindigkeitskoeffizient für sehr kleine Radikale bezeichnet. Als Fitparameter für das gesamte Messsignal wird $k_{\mathrm{t}}^{0}$ allerdings durch das Verhalten von allen im betrachteten Zeitintervall vorliegenden Radikalkettenlängen beeinflusst.

Für Homopolymerisationen von MA, BA und DMA ist in der Abbildung $8.13 k_{\mathrm{t}}^{0}$ bis zu Monomerumsätzen von etwa $20 \%$ dargestellt. Bei $k_{\mathrm{t}}^{0}$ ergibt sich ein ähnliches Verhalten wie beim über alle Kettenlängen gemittelten $\left\langle k_{t}>\right.$. Für alle untersuchten Monomere zeigt sich, wie bereits in den theoretischen Grundlagen vorgestellt und auch bei den Copolymerisationen für $<k_{\mathrm{t}}>$ beobachtet, zu Beginn der Polymerisation ein Plateaubereich mit einem vom Monomerumsatz unabhängigen $k_{\mathrm{t}}^{0}$.

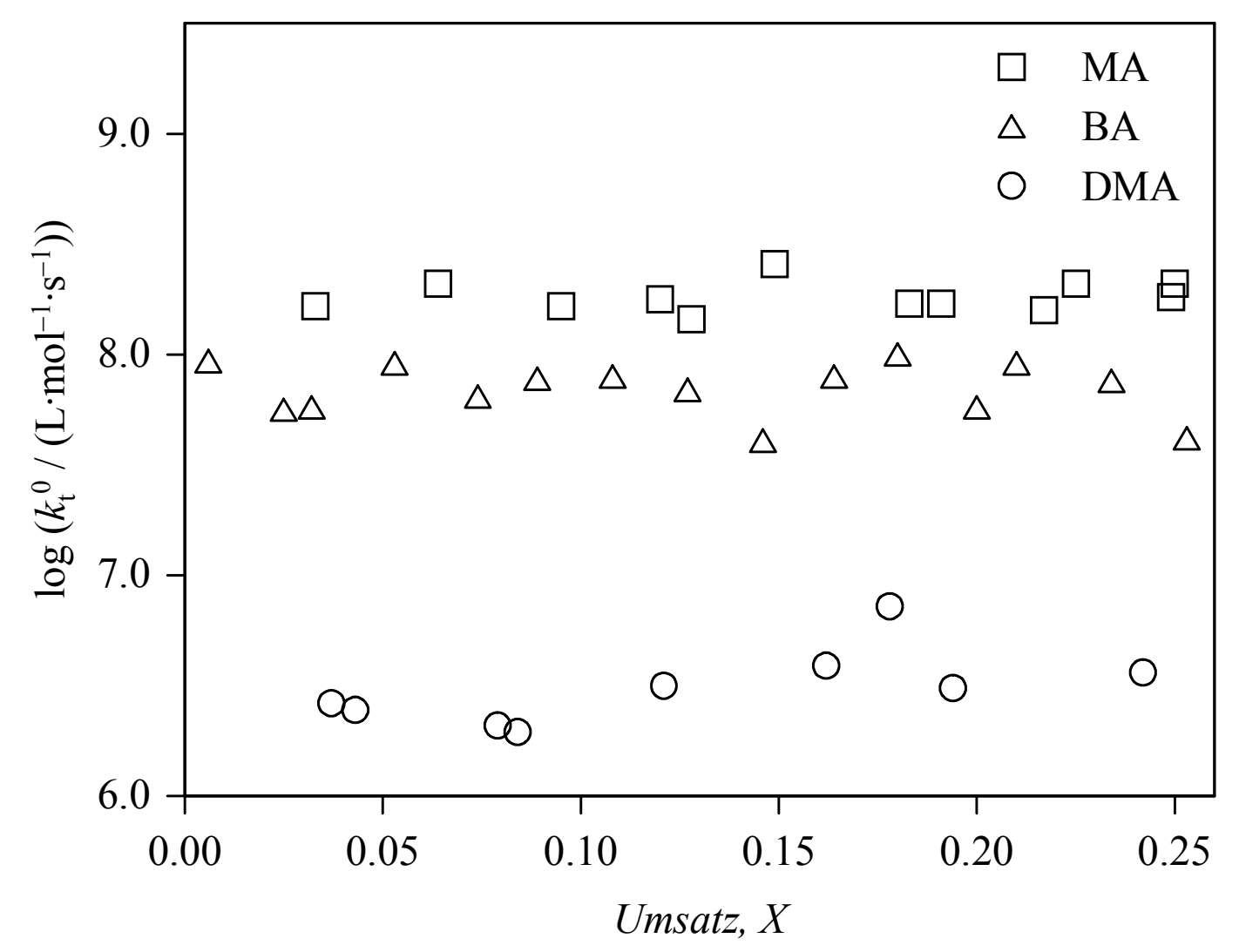

Abb. 8.13: Umsatzabhängigkeit von $\log \left(k_{\mathrm{t}}^{0} /\left(\mathrm{L} \cdot \mathrm{mol}^{-1} \cdot \mathrm{s}^{-1}\right)\right)$ für Polymerisationen von MA, BA und DMA bei $40{ }^{\circ} \mathrm{C} / 1000$ bar. 
Als Parameter für kleine, bewegliche Radikale sollte $k_{\mathrm{t}}^{0}$ erst bei höheren Umsätzen mit großer Zunahme der Viskosität der Reaktionsmischung und dadurch behinderter Translationsdiffusion abnehmen. Dies ist im hier gezeigten Umsatzbereich noch nicht zu erwarten. Vergleicht man die Ergebnisse für die verschiedenen Monomere aus Abbildung 8.13 miteinander, so erkennt man die gleiche Abfolge für $k_{\mathrm{t}}^{0}$, wie sie bereits für die kettenlängengemittelten $\left\langle k_{\mathrm{t}}>\right.$ aus Messungen mit DMPA erhalten wurde (vgl. Abbildung 6.1, Seite 36). Der Wert von MA liegt etwa eine halbe logarithmische Einheit über $k_{\mathrm{t}}^{0}$ für BA und bei DMA ist der Terminierungsgeschwindigkeitskoeffizient nochmals um mehr als eine Einheit niedriger. Die Absolutwerte von $k_{\mathrm{t}}^{0}$ für MA und DMA sollten wegen des Einflusses des Photoinitiators mit den Daten aus Polymerisationen mit MMMP (Abbildung 8.3) verglichen werden. Für diese Monomere ist $k_{\mathrm{t}}^{0}$ jeweils nur ca. 0.4 Einheiten über dem mittleren $<k_{\mathrm{t}}>$ für längere Auswertezeiten, was für eine schwache Kettenlängenabhängigkeit von $k_{\mathrm{t}}$ mit nach Gleichung 8.6 kleinem Parameter $\alpha$ spricht. Dieser Koeffizient sollte dementsprechend für MA, BA und DMA im betrachteten Umsatzbereich in etwa gleich groß sein.

Nicht in die Abbildung eingetragen ist DA. Bereits bei der Diskussion der Abbildung 8.4 wurde erwähnt, dass für dieses Monomer schon bei niedrigen Monomerumsätzen ein deutlicher Einfluss der Signalaufnahmezeit auf das mittlere $\left\langle k_{t}>\right.$ vorliegt. Es ergibt sich also ein größerer Parameter $\alpha$ und auch $k_{\mathrm{t}}^{0}$ passt nicht in die oben beschriebene Abfolge. Zu der hier beschriebenen und in Abbildung 6.1 (Seite 36) dargestellten Reihenfolge von $<k_{\mathrm{t}}>$ und $k_{\mathrm{t}}{ }^{0}$ bei den Acrylaten und Methacrylaten sind noch einige Anmerkungen notwendig. So ist bei einem Vergleich verschiedener Monomere hinsichtlich $\left\langle k_{t}\right\rangle$ darauf $\mathrm{zu}$ achten, dass jeweils dieselbe Kettenlänge untersucht wurde. In Abbildung 6.1 wurde für $<k_{t}>$ von DMA und DA bei $40{ }^{\circ} \mathrm{C} / 1000$ bar ein fast gleich großer Wert eingetragen. Bei Betrachtung der Abbildungen 8.3 und 8.4 wird jedoch klar, dass dies nur für die Auswertung bei gleicher Signalaufnahmezeit der Fall ist. Werden die Signale bis jeweils $0.1 \mathrm{~s}$ analysiert, so ergibt sich

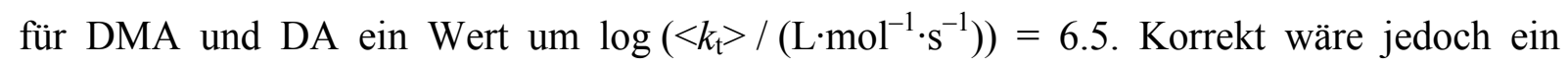
Vergleich bei gleicher Kettenlänge. Aufgrund des deutlich größeren $k_{\mathrm{p}}$ von DA sollten dann die Ergebnisse bei Analyse bis $0.02 \mathrm{~s}$ für DA und $0.5 \mathrm{~s}$ für DMA verglichen werden. In diesem Fall ist $\left\langle k_{t}>\right.$ von DA um etwa eine halbe logarithmische Einheit größer als $\left\langle k_{t}>\right.$ von DMA. Die Bestimmung sowohl von $<k_{\mathrm{t}}>$ als auch von $k_{\mathrm{t}}^{0}$ erfordert die Kenntnis von $k_{\mathrm{p}}$. Später in diesem Kapitel wird auf mögliche Nebenreaktionen hingewiesen, die die Ermittlung 
von $k_{\mathrm{p}}$ erschweren können. In Abschnitt 8.3.4 wird das abweichende Verhalten einiger Acrylate diskutiert.

Der folgende Teil dieses Kapitels stellt nun die Ergebnisse für $\alpha$ von einigen Monomeren bis zu hohen Monomerumsätzen vor.

\subsubsection{Parameter $\alpha$ bei niedrigen Monomerumsätzen}

In Abbildung 8.14 sind die aus der Anpassung von Gleichung 8.8 erhaltenen Ergebnisse für $\alpha$ für Homopolymerisationen von MA, BA, DMA und MMA bis zu Monomerumsätzen von etwa $20 \%$ gezeigt. Alle Daten stammen aus Experimenten bei $40{ }^{\circ} \mathrm{C} / 1000$ bar, nur für MMA wurde wegen des günstigeren Signal-Rausch-Verhältnisses ein Druck von 2000 bar gewählt.

Für MA gibt es in der Literatur Werte für diesen Parameter von $\alpha=0.32$ [3] und $\alpha=0.35$ 0.36 (für Kettenlängen $i$ zwischen 50 und 100) [9]. Der letztere Wert lässt sich allerdings nicht mit dem in dieser Arbeit erhaltenen Ergebnis vergleichen, da mit der hier verwendeten Methode derart kurze Ketten und entsprechend kurze Aufnahmezeiten nicht untersucht werden können. Der Unterschied zu dem in Referenz [3] aufgeführten $\alpha$ lässt sich damit erklären, dass dort für $k_{\mathrm{t}}^{0}$ ein Wert von $7 \cdot 10^{8} \mathrm{~L} \cdot \mathrm{mol}^{-1} \cdot \mathrm{s}^{-1}$ angenommen wurde, während sich hier bei der Auswertung nach Gleichung 8.8 ein $k_{\mathrm{t}}^{0}$ für MA von etwa $2 \cdot 10^{8} \mathrm{~L} \cdot \mathrm{mol}^{-1} \cdot \mathrm{s}^{-1}$ ergibt. (vgl. Abb. 8.14). Mit der in Abbildung 8.11 illustrierten Korrelation der beiden Parameter ist somit auch der in dieser Arbeit erhaltene niedrigere Wert für $\alpha$ nachvollziehbar. In Abbildung 8.11 wurde gezeigt, dass bei niedrigen Monomerumsätzen innerhalb einer Messreihe ein vergleichsweise hoher Wert von $k_{\mathrm{t}}^{0}$ auch ein hohes $\alpha$ bedeutet. Würde man bei der Anpassung mit Gleichung $8.8 k_{\mathrm{t}}^{0}$ bei einem größeren Wert fixieren, ergäbe sich dementsprechend auch für $\alpha$ eine Übereinstimmung mit dem in Referenz [3] angegebenen Wert von 0.32 .

Für BA gibt de Kock einen Wert von $\alpha=0.17-0.19$ an [9], allerdings wie bei MA für Kettenlängen zwischen 50 und 100, was sich, wie bereits erwähnt, nicht mit den in dieser Arbeit erhaltenen Ergebnissen vergleichen lässt. 


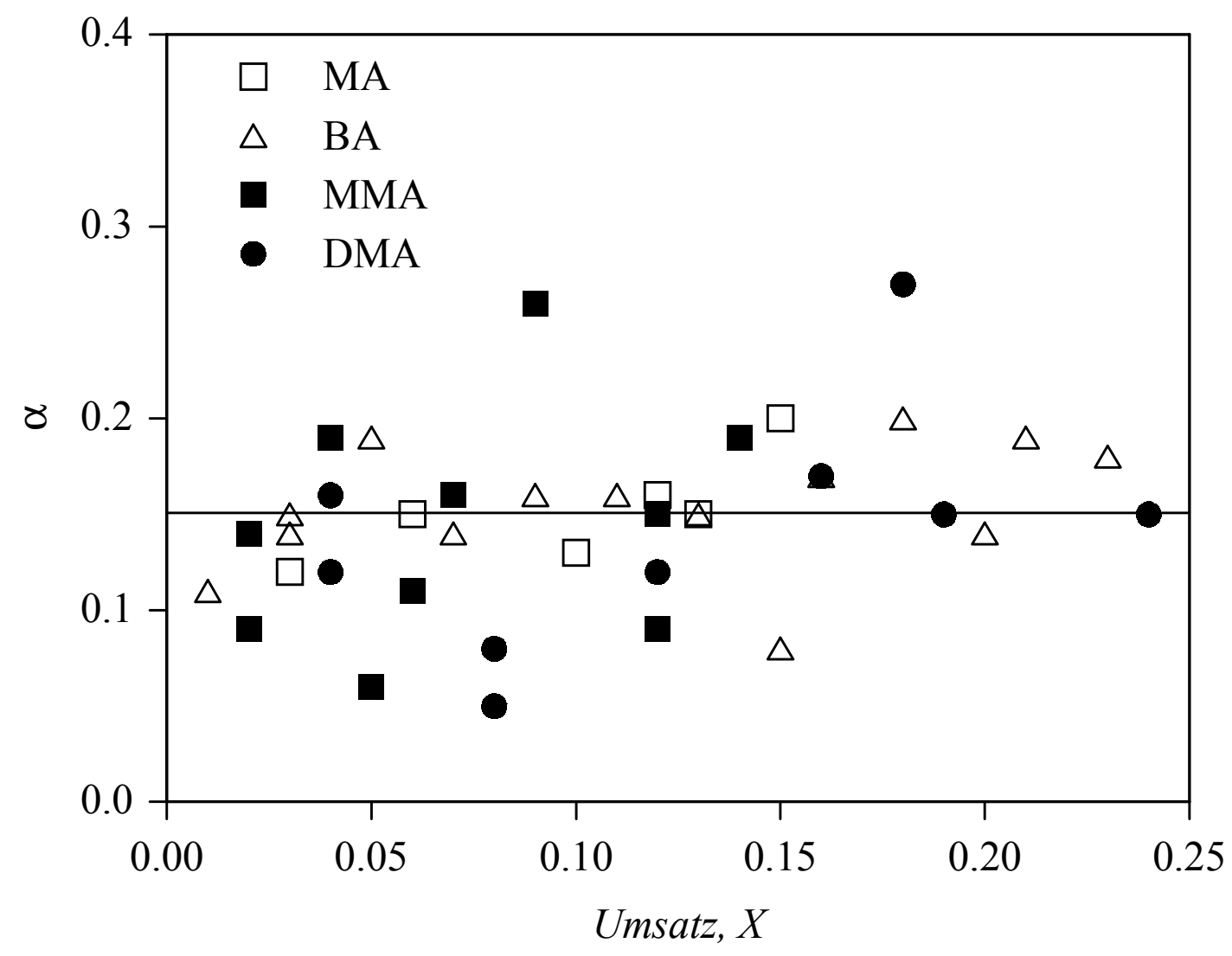

Abb. 8.14: Exponent $\alpha$ aus der Anpassung von Gleichung 8.8 an SP-PLP-Signale für MA, BA und DMA bei $40{ }^{\circ} \mathrm{C} / 1000$ bar sowie für MMA bei $40{ }^{\circ} \mathrm{C} / 2000$ bar. Die horizontale Linie kennzeichnet den Mittelwert von $\alpha=0.15$.

Die Literaturwerte für MMA von $\alpha=0.15$ [21] und $\alpha=0.16-0.17$ [22] sind in guter Übereinstimmung mit den in Abbildung 8.15 gezeigten Daten. In diesem Bereich liegen auch die publizierten Ergebnisse für Styrol ( $\alpha=0.24$ [21] und $\alpha=0.17$ [22]).

Der Mittelwert über alle in Abbildung 8.14 dargestellten Daten für MA, BA, MMA und DMA beträgt $\alpha=0.15$ und ist als horizontale Linie verdeutlicht. Dieses Ergebnis ist nahe dem von Friedman und O'Shaugnessy [23, 24] vorhergesagten theoretischen Wert von $\alpha=0.16$ für chemisch- und diffusionskontrollierte Reaktionen in verdünnten Lösungen mit hinreichend großen, linearen Radikalen $(i>3000)$, bei denen sich die Radikalfunktion am Kettenende oder nahe dem Kettenende befindet. Für den Fall, dass bei einem der terminierenden Radikale das aktive Zentrum eher in der Mitte und beim anderen am Kettenende ist, wird $\alpha=0.27$ angenommen. Schließlich sollte dann, wenn die Radikalfunktion bei beiden Radikalen in der Mitte der Kette zu finden ist, ein Exponent von $\alpha=0.43$ resultieren [23]. Solche Radikale werden in diesem Kapitel als „mid-chain“ Radikale bezeichnet. 
Für die in Abbildung 8.14 gezeigten Untersuchungen ergibt sich ein Exponent $\alpha<0.2$ auch für Umsätze bis zu 15 oder 20 \%, was bei Polymerisationen eindeutig nicht mehr als Bereich verdünnter Lösungen zu bezeichnen ist. Im Folgenden soll das Verhalten bei mittleren und höheren Umsätzen in die Diskussion mit einbezogen werden. Dabei werden Acrylate und Methacrylate getrennt behandelt.

\subsection{3 $\alpha$ für Methacrylate bis zu höheren Monomerumsätzen}

Bei der Diskussion der Abbildungen 8.3 und 8.4 wurde bereits erwähnt, dass bei höheren Monomerumsätzen die Differenz des mittleren $k_{\mathrm{t}}$ für kurze und lange Signalaufnahmezeiten zunimmt. Das deutet auf ein Ansteigen des Exponenten $\alpha$ mit dem Umsatz hin. In Abbildung 8.15 ist $\alpha$ für Homopolymerisationen von DMA bei $40{ }^{\circ} \mathrm{C} / 1000$ bar und von MMA bei $40{ }^{\circ} \mathrm{C} / 2000$ bar als Funktion des Umsatzes aufgetragen.

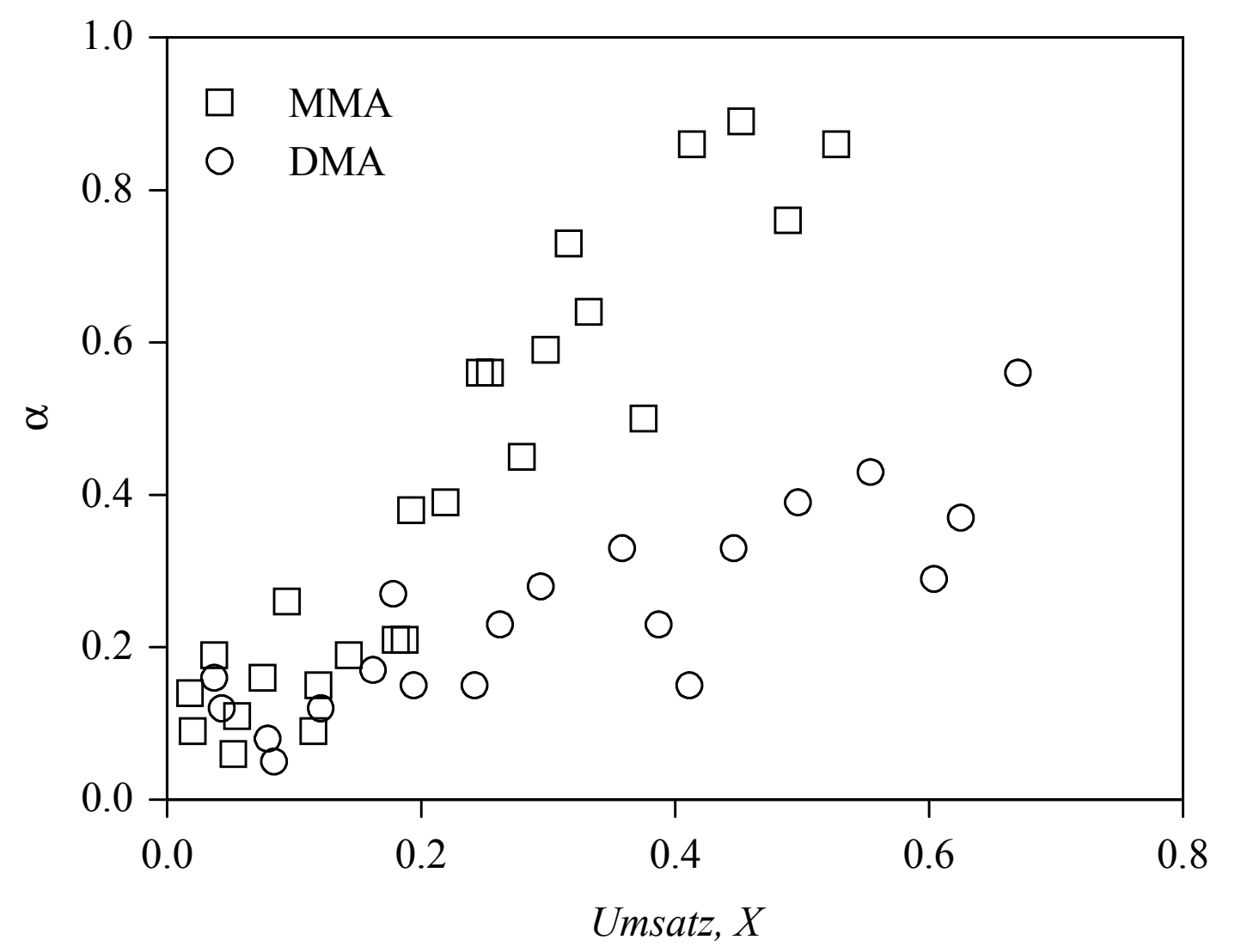

Abb. 8.15: Der Exponent $\alpha$ als Funktion des Monomerumsatzes für Homopolymerisationen von DMA bei $40{ }^{\circ} \mathrm{C} / 1000$ bar und von MMA bei $40^{\circ} \mathrm{C} / 2000$ bar. 
Für beide Monomere aus der Abbildung ergibt sich für niedrige Umsätze, wie schon in Abbildung 8.14 gezeigt, ein $\alpha$ um 0.15 . Ab etwa $15 \%$ Umsatz erhöht sich der Exponent mit Einsetzen des Geleffekts merklich. Dieses Verhalten ist im Fall von MMA noch stärker ausgeprägt, so dass $\alpha$ schließlich bei einem Umsatz von etwa $50 \%$ nahe eins ist.

Das deutliche Ansteigen von $\alpha$ bei größeren Monomerumsätzen weist auf eine Änderung im Mechanismus der Diffusionskontrolle des Terminierungsgeschwindigkeitskoeffizienten hin. Im Plateaubereich bei niedrigen Umsätzen wird $k_{\mathrm{t}}$ durch Segmentdiffusion kontrolliert. In diesem Bereich ist $\alpha$ klein und zeigt die mit zunehmender Kettenlänge $i$ verringerte Wahrscheinlichkeit, dass beim Überlappen von zwei Makroradikalen auch die Radikalfunktionen am Kettenende miteinander in Kontakt kommen. Bei höheren Umsätzen gerät $k_{\mathrm{t}}$ zunehmend unter Translationsdiffusionskontrolle und das größere $\alpha$ bedeutet eine starke Einschränkung der Bewegung von langen Ketten. Für durch Translationsdiffusion kontrollierte Terminierungsreaktionen wird für nicht miteinander verstrickte Radikale („nonentangled regime“) in halbverdünnter oder konzentrierter Lösung ein $\alpha$ von 0.5 vorausgesagt [17]. Die hier ermittelten Werte für MMA liegen jedoch deutlich über 0.5 bei höheren Umsätzen. Entsprechend den Erläuterungen zu Gleichung 8.7 wurde von de Gennes [17] für miteinander verstrickte Makroradikale (,entangled regime“) ein Exponent von 1.5 vermutet. Welcher Bereich bei den in der Abbildung 8.15 dargestellten Ergebnissen vorliegt, lässt sich anhand der Daten nicht entscheiden. Außerdem wurde bei den Untersuchungen zur MMAHomopolymerisation (Kapitel 6) davon ausgegangen, dass die Terminierungsreaktion bei Monomerumsätzen größer 50 \% durch Reaktionsdiffusion bestimmt wird. In diesem Bereich sollte $k_{\mathrm{t}}$ kettenlängenunabhängig sein. Da in dieser Arbeit keine Daten für noch höhere Umsätze bestimmt werden konnten, bleibt unklar, ob $\alpha$ tatsächlich ein Maximum durchläuft und wieder abfällt.

\subsection{4 $\alpha$ für Acrylate bis zu höheren Monomerumsätzen}

Entsprechend dem vorherigen Abschnitt werden nun auch für die Acrylate die Ergebnisse bis zu höheren Umsätzen betrachtet. In die Diskussion wird als weiteres Monomer DA miteinbezogen. Abbildung 8.16 stellt $\alpha$ als Funktion des Umsatzes für MA, BA und DA bei $40{ }^{\circ} \mathrm{C} / 1000$ bar dar. 


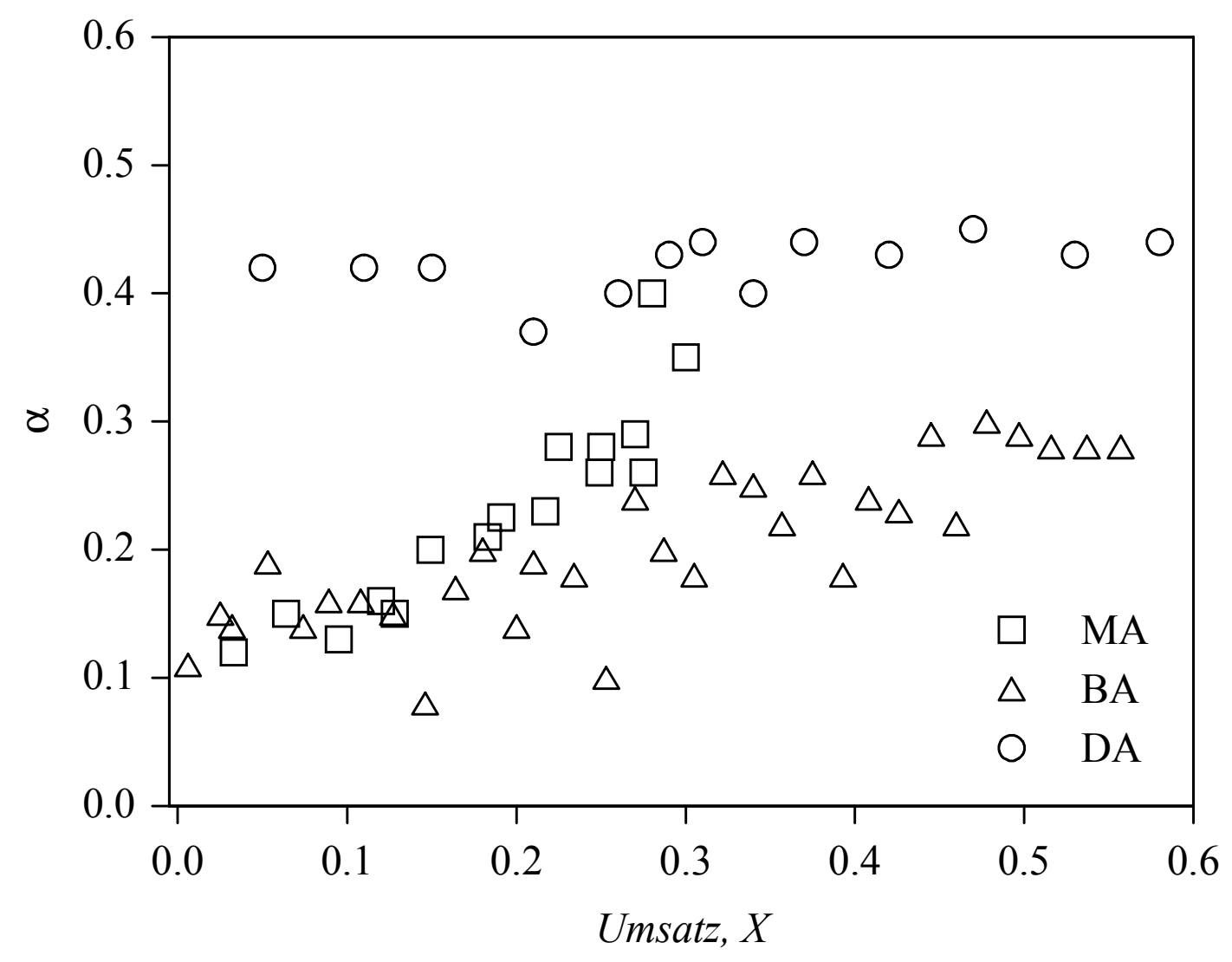

Abb. 8.16: Der Exponent $\alpha$ als Funktion des Monomerumsatzes für Homopolymerisationen von MA, BA und DA bei $40^{\circ} \mathrm{C} / 1000$ bar.

MA und BA zeigen das schon für MMA und DMA aus Abbildung 8.14 bekannte Verhalten. Im Anfangsbereich bis etwa $15 \%$ Monomerumsatz ergibt sich ein $\alpha$ um 0.15 und der Exponent steigt mit zunehmendem Umsatz an. Auffällig ist jedoch, dass zwischen 20 und $30 \%$ Monomerumsatz $\alpha$ für MA jeweils über $\alpha$ für BA liegt. Bei MA ist der Anstieg von $\alpha$ mit dem Umsatz nach dem Anfangsbereich deutlich stärker. Es kann vermutet werden, dass bei MA ähnlich zu MMA bei mittleren Monomerumsätzen andere Diffusionsprozesse für die Terminierungsreaktion bestimmend werden. Bei diesen Monomeren fällt auch das mittlere $<k_{\mathrm{t}}>$ im Bereich des Geleffekts besonders stark ab.

Für das auch in der Abbildung 8.16 gezeigte DA jedoch bleibt $\alpha$ nahe 0.4 über dem gesamten Umsatzbereich. In Kapitel 7 wurde bereits das Verhalten des kettenlängengemittelten $<k_{\downarrow}>$ für DA bei den gleichen experimentellen Bedingungen diskutiert. Das mittlere $\left\langle k_{t}\right\rangle$ ist entsprechend den dort besprochenen Untersuchungen umsatzunabhängig und somit ist ein konstantes $\alpha$ nicht unerwartet. Schwieriger zu verstehen ist der hohe Absolutwert von $\alpha$ bei diesem Monomer. Da sich DA von den anderen in Abbildung 8.16 dargestellten Acrylaten 
durch den deutlich größeren Esterrest unterscheidet, soll im Folgenden der Einfluss der Seitenkette auf $\alpha$ untersucht werden. Deshalb werden in Abbildung 8.17 einige Ergebnisse für Monomere mit ähnlich voluminösem Esterrest, Ethylhexylacrylat und Isodecylacrylat, gezeigt.

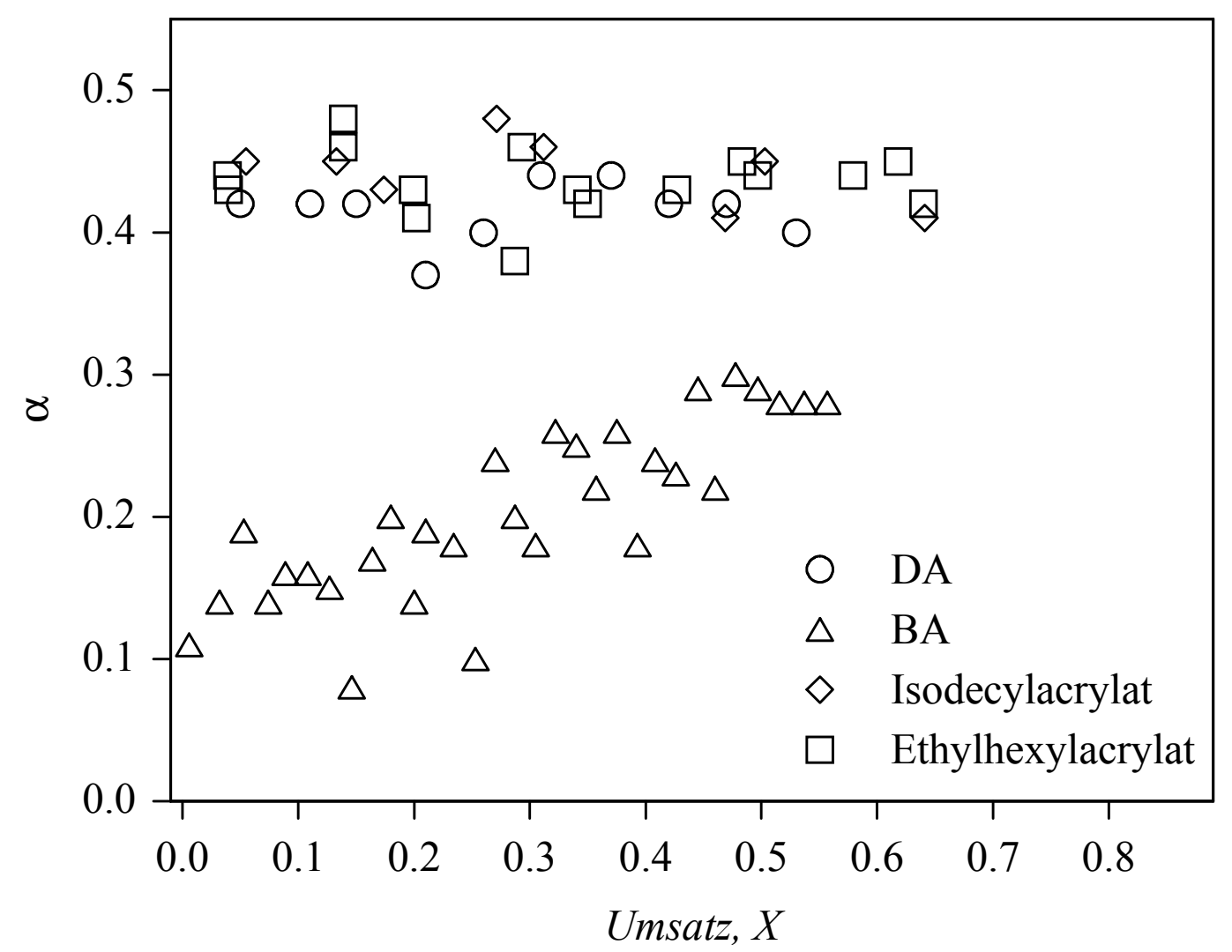

Abb. 8.17: Der Exponent $\alpha$ als Funktion des Monomerumsatzes für Homopolymerisationen von DA, BA, Isodecylacrylat und Ethylhexylacrylat bei $40^{\circ} \mathrm{C} / 1000$ bar.

Aus der Abbildung geht hervor, dass für Ethylhexylacrylat und Isodecylacrylat im Rahmen der Messungenauigkeit eine Kettenlängenabhängigkeit von $k_{\mathrm{t}}$ wie bei DA vorliegt. Der Exponent $\alpha$ ist auch bei diesen Monomeren vom Monomerumsatz unabhängig und liegt bei etwa 0.4.

Gemäß den in Kapitel 8.3.2 vorgestellten theoretischen Überlegungen würde eine Terminierung von Radikalen mit der Radikalfunktion in der Mitte der Kette zu einem $\alpha$ mit dem Wert 0.43 führen. Ein hoher Anteil an Radikalen mit dem aktiven Zentrum in der Mitte würde auch einen bedeutsamen Einfluss auf den Wachstumsgeschwindigkeitskoeffizienten $k_{\mathrm{p}}$ ausüben. Radikale dieser Art können durch Transferreaktionen auf das Polymer oder auf das wachsende Makroradikal entstehen.

Bei den hier erwähnten Acrylaten (DA, Ethylhexylacrylat und Isodecylacrylat) wäre eine Übertragung der Radikalfunktion auf den großen Esterrest desselben Kettenglieds denkbar. 
Diese Reaktion könnte über einen 6-gliedrigen Übergangszustand verlaufen. Dabei würden wachsende Radikale gebildet, bei denen sich das aktive Zentrum nicht am Ende befindet. Allerdings hätte das Produkt eines solchen intramolekularen Transfers die Radikalfunktion immer noch nahe dem Kettenende, was gemäß der Theorie [23, 24] eher zu einem $\alpha$ von 0.16 führen sollte.

Diese Hypothese der intramolekularen Übertragung sollte in einigen Experimenten überprüft werden. Als Beleg für das Auftreten einer solchen Reaktion können ${ }^{13} \mathrm{C}$-NMR-Analysen entsprechender Polymerproben dienen. Falls eine intramolekulare Übertragung stattfindet, sollten sich mit dieser Technik tertiäre C-Atome in der Seitenkette und damit Verzweigungen nachweisen lassen. Solche Analysen konnten jedoch wegen unzureichender Löslichkeit des bei der SP-PLP-Technik produzierten sehr hochmolekularen Polymers in den zur Verfügung stehenden NMR-Lösungsmitteln nicht durchgeführt werden. Eine bessere Löslichkeit ließe sich durch chemisch initiierte Polymerisationen oder die Wahl einer höheren Laserfrequenz bei PLP erreichen. Auch durch die Zugabe eines Transferagens könnte die Polymermolmasse gesenkt werden. In jedem dieser Fälle würden jedoch die Reaktionsbedingungen stark verändert werden, so dass die dann erhaltenen NMR-Daten für die SP-PLP-Experimente von nur eingeschränkter Aussagekraft sind.

Als Alternative zu den NMR-Analysen wurden deshalb weitere Monomere mit verschiedenem Esterrest untersucht. Zunächst wurden Messungen mit Hexylacrylat durchgeführt, bei dem nach einer Transferreaktion auf die Seitenkette das aktive Zentrum immer noch nahe dem Kettenende (jetzt nahe dem Ende der Seitenkette) läge und außerdem im Vergleich zum Ethylhexylacrylat noch weniger abgeschirmt wäre. Im Vergleich dazu sollte aus sterischen Gründen beim Cyclohexylacrylat keine Übertragung auf den Cyclohexyl-Ring möglich sein. In diesem Fall kann kein 6-gliedriger Übergangszustand gebildet werden. In der Abbildung 8.18 sind die Ergebnisse dargestellt, die für SP-PLP-Experimente mit diesen Monomeren bei $40{ }^{\circ} \mathrm{C} / 1000$ bar erhalten wurden. 


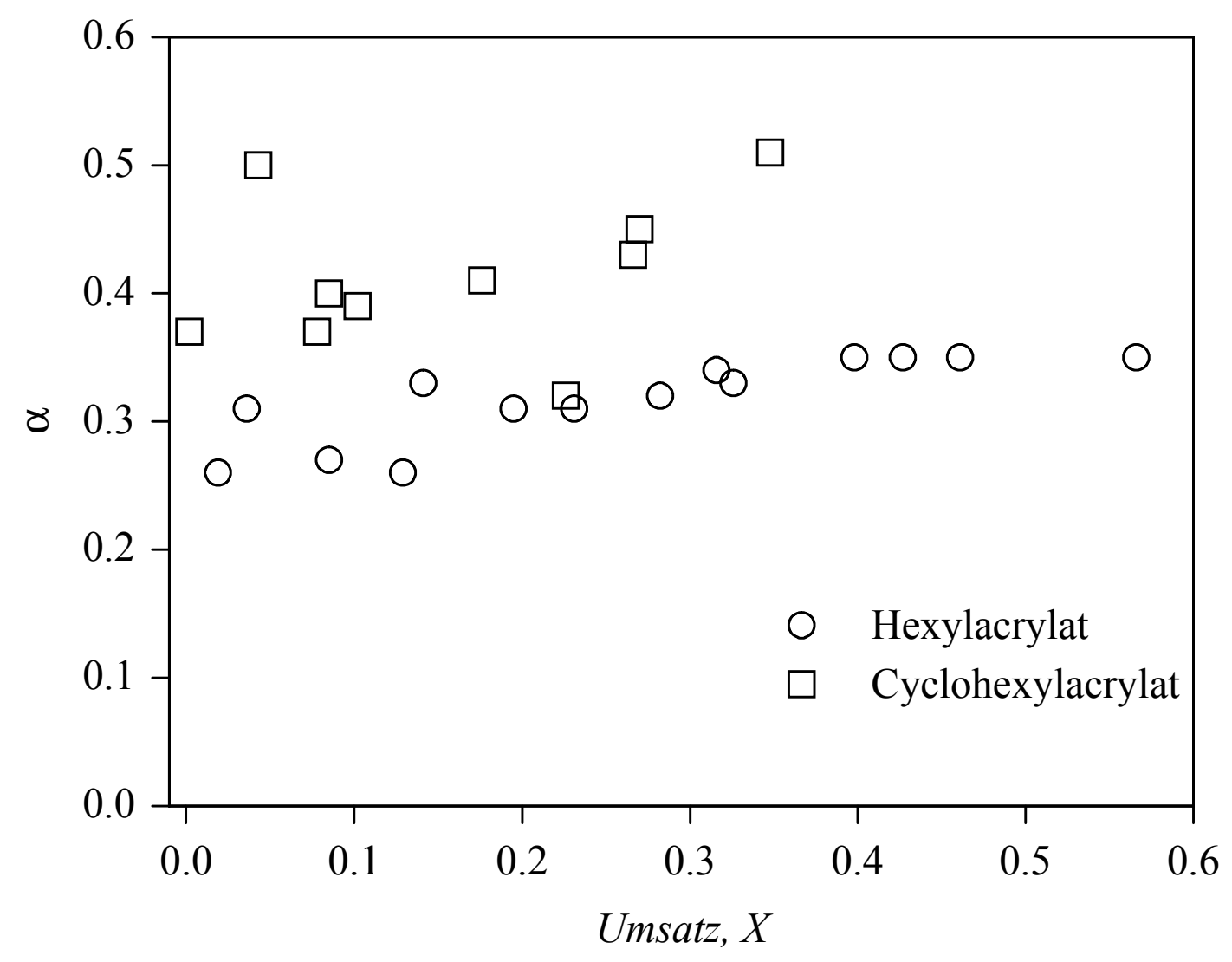

Abb. 8.18: Der Exponent $\alpha$ als Funktion des Monomerumsatzes für Homopolymerisationen von Hexylacrylat und Cyclohexylacrylat bei $40{ }^{\circ} \mathrm{C} / 1000$ bar.

Die Werte für Hexylacrylat scheinen die Hypothese zunächst zu bestätigen. Es ergibt sich ein $\alpha$ um 0.3, was deutlich über dem Wert für MA und BA, aber auch unter dem Wert für DA liegt. Bei einer intramolekularen Transferreaktion wäre im Fall von Hexylacrylat die Radikalfunktion nicht mehr am Kettenende, aber auch nicht weit in der Mitte. Ein $\alpha$, das zwischen den theoretischen Werten von 0.16 für Reaktionen von „mid-chain“ Radikalen und 0.43 für Reaktionen von „end-chain“ Radikalen miteinander liegt, ist somit verständlich.

Die Experimente mit Cyclohexylacrylat waren wegen eines schnellen Auftretens von Inhomogenitäten schwierig, was sich auch in der erheblichen Streuung der Ergebnisse widerspiegelt. Trotzdem ist klar zu erkennen, dass $\alpha$ für Cyclohexylacrylat im Mittel über dem Wert für Hexylacrylat liegt. Falls ein intramolekularer Transfer auf dasselbe Kettenglied stattfinden würde, müsste es jedoch umgekehrt sein.

Einen weiteren Hinweis, ob eine intramolekularen Reaktion mit der Seitenkette stattfindet, kann der Vergleich von BA mit tert-Butylacrylat, das als weiteres Monomer untersucht wurde, liefern. Die Ergebnisse für diese beiden Monomere sind in Abbildung 8.19 veranschaulicht. Ähnlich wie bei den Polymerisationen aus Abbildung 8.18 sollte eine Übertragung bei 
tert-Butylacrylat nicht möglich sein, während BA eine solche Reaktion eingehen könnte. Bei BA wäre dann allerdings das aktive Zentrum trotzdem am Kettenende und wiederum würde $\alpha=0.16$ resultieren. Die schon in Abbildung 8.16 gezeigten BA-Daten können somit die Hypothese ohnehin weder bestätigen noch widerlegen.

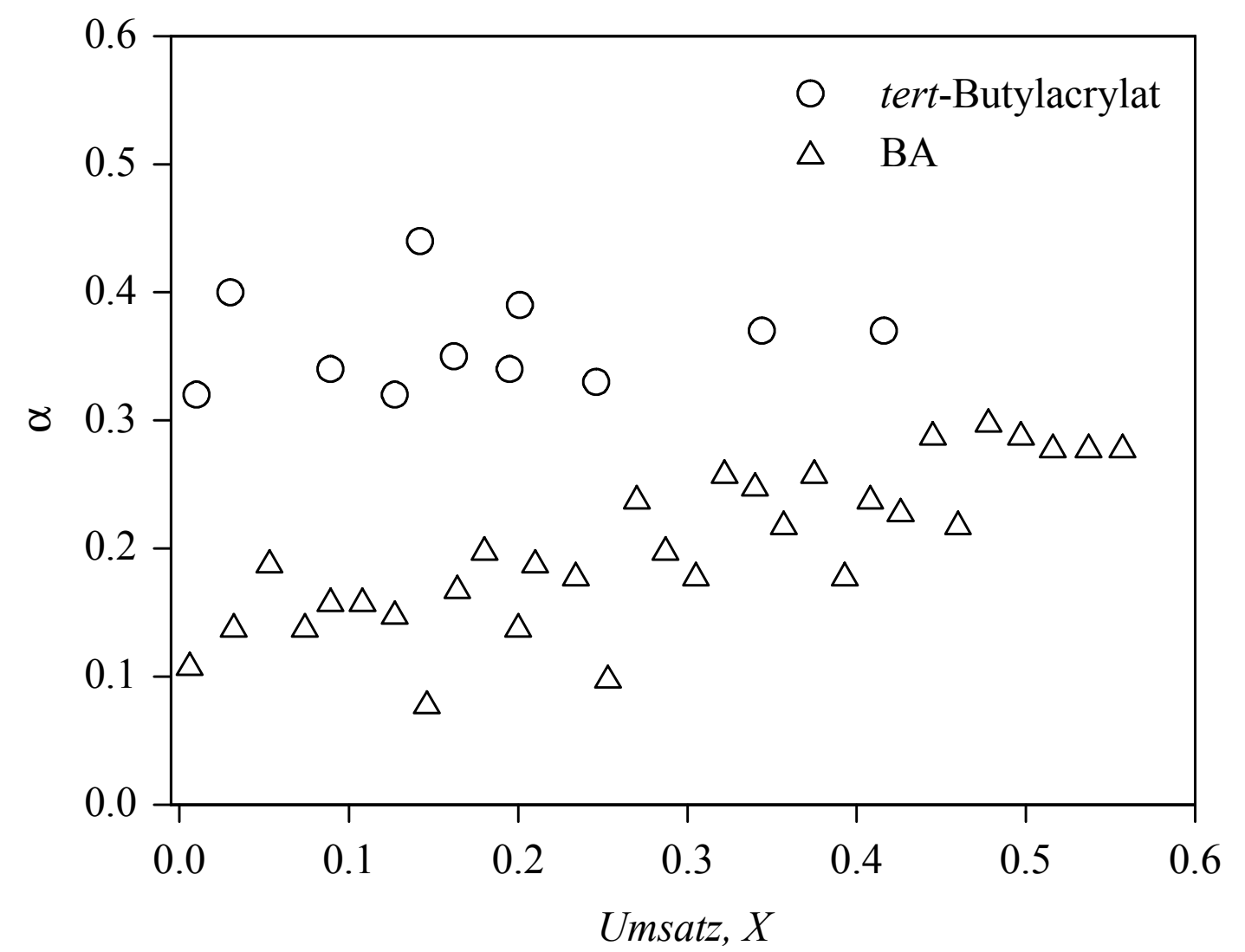

Abb. 8.19: Der Exponent $\alpha$ als Funktion des Monomerumsatzes für Homopolymerisationen von BA und tert-Butylacrylat bei $40^{\circ} \mathrm{C} / 1000$ bar.

Das Ergebnis für tert-Butylacrylat hingegen ist eindeutig. Es ergibt sich ein Exponent um 0.35, obwohl hier keine Übertragungsreaktion auf die Esterseitenkette über einen 6-gliedrigen Übergangszustand stattfinden kann. Zusammen mit den in Abbildung 8.18 gezeigten Daten für Hexylacrylat und Cyclohexylacrylat zeigen diese Untersuchungen also, dass sich ein solcher Transfer auf den Esterrest als Grund für die größere Kettenlängenabhängigkeit von $k_{\mathrm{t}}$ bei einigen Monomeren ausschließen lässt.

Außer der Übertragung auf den Esterrest desselben Kettengliedes kommt eine weitere Transferreaktion für die Bildung von Radikalen mit aktivem Zentrum in der Mitte der Kette infrage. Die Ergebnisse legen nahe, dass die Radikalfunktion intra- oder intermolekular auf andere Monomereinheiten der wachsenden Kette durch Abstraktion eines Wasserstoffatoms 
übertragen werden könnte. Dies ist bei Acrylaten wegen der höheren Reaktivität und Flexibilität der Makroradikale wahrscheinlicher als bei Methacrylaten. Auch auf diese Weise können verzweigte Polymere entstehen. Der intramolekulare Transfer verläuft meist über einen 6-gliedrigen Übergangszustand mit den letzten 3 Monomereinheiten und wird als „,backbiting“ bezeichnet [25, 26]. Über einen solchen Mechanismus entstehen Kurzkettenverzweigungen. Es ist jedoch auch eine Übertragung auf weiter vom aktiven Zentrum entfernte Kettenglieder mit der Bildung von Langkettenverzweigungen vorstellbar, wobei sich eine solche Reaktion nicht vom Transfer zum Polymer unterscheidet. Das Auftreten von Transferreaktionen wurde bereits als Erklärung für hohe Reaktionsordnungen des Monomers bei Polymerisationen von $n$-Alkylacrylaten vorgeschlagen [27] und von einigen Arbeitsgruppen bei Polymerisationen verschiedener Acrylate, wie BA [28, 29, 30, 31, 32], Cyclohexylacrylat [33], Ethylhexylacrylat [34] und Phenylacrylat [35] beobachtet. Während Ahmad et al. [28] für BA fanden, dass es sich bei den Verzweigungen mehrheitlich um Langkettenverzweigungen handelt, deuteten die Untersuchungen von Plessis et al. [30, 31, 32] bei demselben Monomer auf eine Dominanz von Kurzkettenverzweigungen hin.

In beiden Fällen ist für die Wahrscheinlichkeit einer solchen Reaktion die Anzahl der zur Verfügung stehenden Wasserstoffatome sowie die Stabilität der neu gebildeten Radikale von Bedeutung. So können etwa bei DA im Vergleich zu etwa BA deutlich mehr H-Atome abstrahiert werden. Im Fall von tert-Butylacrylat wird eine Radikalfunktion im Esterrest besser stabilisiert als bei BA. Im Folgenden werden weitere Experimente zur Untersuchung des Zusammenhangs zwischen der Bildung von „,mid-chain“ Radikalen und der Größe der Kettenlängenabhängigkeit von $k_{\mathrm{t}}$ vorgestellt.

In Abbildung 8.20 ist der Parameter $\alpha$ als Funktion des Monomerumsatzes für Polymerisationen von Phenylacrylat und Benzylacrylat dargestellt. Bei diesen Monomeren ist die Abstraktion eines Wasserstoffatoms vom Esterrest wegen der geringen Stabilität der entstehenden Radikale sehr unwahrscheinlich. Es stehen also nur die wenigen Wasserstoffatome aus der Hauptkette für Übertragungsreaktionen zur Verfügung. Dementsprechend sollte die Konzentration von „,mid-chain“ Radikalen gering und damit der Exponent $\alpha$ niedrig sein.

Ähnlich wie bei Cyclohexylacrylat waren die Experimente mit Phenylacrylat und Benzylacrylat aufgrund von leicht auftretenden Inhomogenitäten der Reaktionsmischung schwierig. Trotzdem ist aus den Daten deutlich ersichtlich, dass die Kettenlängenabhängigkeit von $k_{\mathrm{t}}$ bei niedrigen Umsätzen wie vorausgesagt gering ist und mit dem Umsatz ansteigt. Die 
beiden Monomere verhalten sich somit wie BA oder MA, unterscheiden sich aber deutlich von den anderen Monomeren mit großem Esterrest.

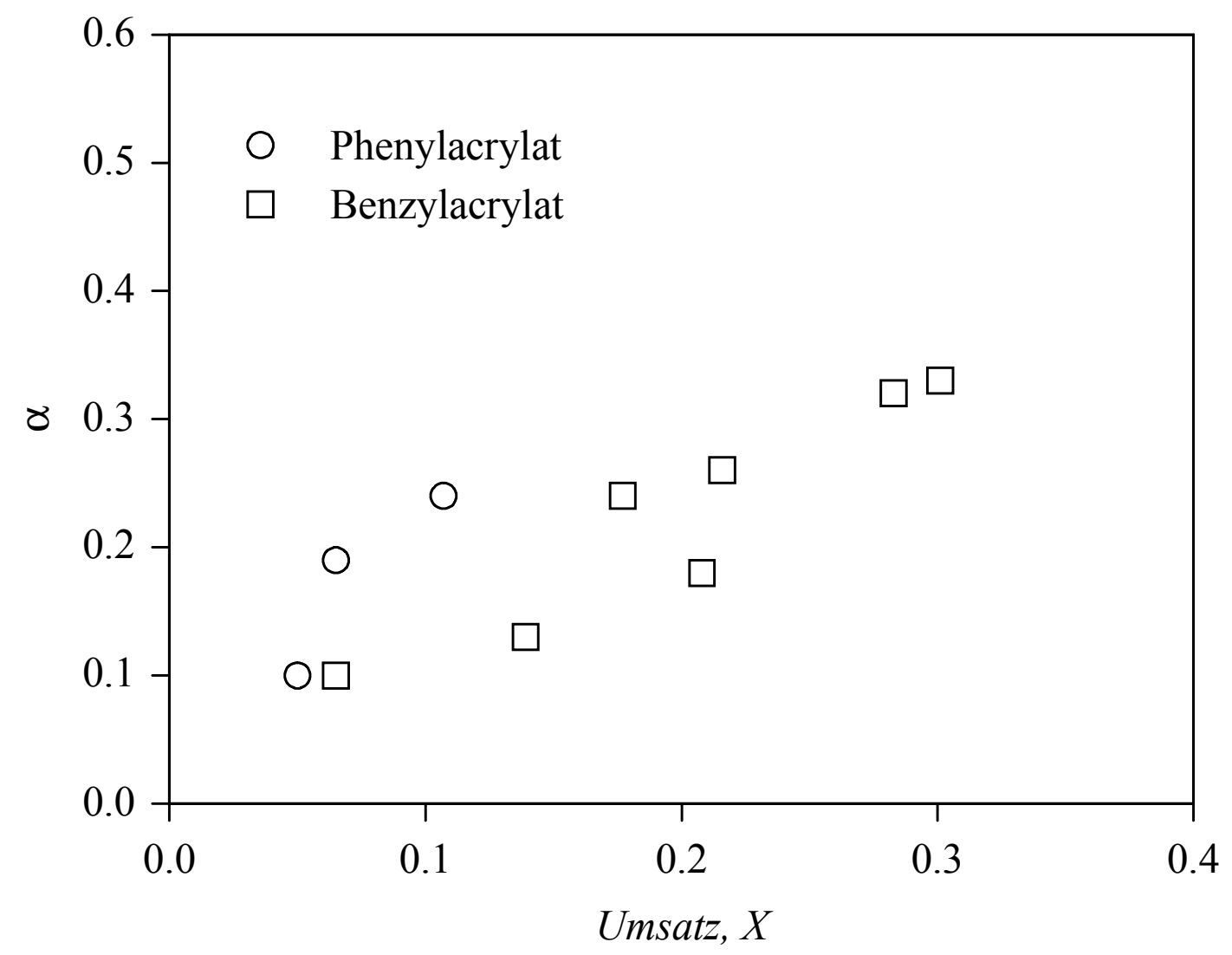

Abb. 8.20: Der Exponent $\alpha$ als Funktion des Monomerumsatzes für Homopolymerisationen von Phenylacrylat und Benzylacrylat bei $40^{\circ} \mathrm{C} / 1000$ bar.

Die hier gezeigten Daten bestätigen somit die Hypothese einer Beteiligung von Radikalen mit aktivem Zentrum in der Mitte der Kette. An dieser Stelle kann zudem ausgeschlossen werden, dass allein die Größe des Esterrests durch eine Abschirmung der Radikalfunktion die Kettenlängenabhängigkeit bestimmt. In einem solchen Fall müssten die Ergebnisse für Phenylacrylat und Benzylacrylat eher denen von Cyclohexylacrylat oder von anderen Monomeren mit voluminöser Esterseitenkette ähneln. Außerdem dürfte es dann keinen großen Unterschied zwischen den oben gezeigten Ergebnissen für DA und DMA geben.

Unter Berücksichtigung einer Transferreaktion zur Bildung von Makroradikalen mit dem aktiven Zentrum in der Mitte der Kette lässt sich ein kinetisches Schema aufstellen, in dem bei den Terminierungsreaktionen jeweils Rekombination und Disproportionierung berücksichtigt sind. Der Übertragungsschritt wird als ,interner Transfer“ bezeichnet, unabhängig davon, ob Lang- oder Kurzkettenverzweigungen gebildet werden. 


$\begin{array}{lll}\text { Initiierung: } & \mathrm{I} \cdot+\mathrm{M} \rightarrow \mathrm{R}_{\mathrm{i}} \cdot & k_{\mathrm{p}} \\ \text { Wachstum: } & \mathrm{R}_{\mathrm{i}} \cdot \mathrm{M} \rightarrow \mathrm{R}_{\mathrm{i}+1^{\cdot}} & k_{\mathrm{p}} \\ \text { Interner Transfer: } & \mathrm{R}_{\mathrm{i}} \cdot \rightarrow \mathrm{R}_{\mathrm{i} \text { mid }} \cdot & k_{\text {itr }} \\ \text { Wachstum tertiäres Radikal: } & \mathrm{R}_{\mathrm{i} \text { mid }}+\mathrm{M} \rightarrow \mathrm{R}_{\mathrm{i}+1^{\cdot}} & k_{\mathrm{p} \text { mid }} \\ \text { Terminierung: } & \mathrm{R}_{\mathrm{i}} \cdot+\mathrm{R}_{\mathrm{j}} \cdot \rightarrow \mathrm{P}_{\mathrm{i}+\mathrm{j}} & k_{\mathrm{t}} \\ & \mathrm{R}_{\mathrm{i}} \cdot+\mathrm{R}_{\mathrm{j}} \cdot \rightarrow \mathrm{P}_{\mathrm{i}}+\mathrm{P}_{\mathrm{j}} & k_{\mathrm{t}} \\ & \mathrm{R}_{\mathrm{i} \text { mid }}+\mathrm{R}_{\mathrm{j} \text { mid }} \rightarrow \mathrm{P}_{\mathrm{i}+\mathrm{j}} & k_{\mathrm{t} \text { mid }} \\ & \mathrm{R}_{\mathrm{i} \text { mid }}+\mathrm{R}_{\mathrm{j} \text { mid }} \rightarrow \mathrm{P}_{\mathrm{i}}+\mathrm{P}_{\mathrm{j}} & k_{\mathrm{tmid}} \\ & \mathrm{R}_{\mathrm{i}} \cdot+\mathrm{R}_{\mathrm{j} \text { mid }} \rightarrow \mathrm{P}_{\mathrm{i}+\mathrm{j}} & k_{\mathrm{t} \text { mid_n }} \\ & \mathrm{R}_{\mathrm{i}} \cdot+\mathrm{R}_{\mathrm{j} \text { mid }} \rightarrow \mathrm{P}_{\mathrm{i}}+\mathrm{P}_{\mathrm{j}} & k_{\mathrm{t} \text { mid_n }}\end{array}$

Es erscheint interessant, die Abnahme der Monomerkonzentration nach einem Laserpuls entsprechend dem vorgestellten kinetischen Schema zu modellieren, z. B. mit dem Programmpaket PREDICI ${ }^{\circledR}$. Die Anzahl der unbekannten Koeffizienten ist jedoch sehr groß, da bei jeder Terminierungsreaktion die Kettenlängenabhängigkeit von $k_{\mathrm{t}}$ mit weiteren Parametern berücksichtigt werden muss. Problematisch ist ebenso die Verwendung von Wachstumsgeschwindigkeitskoeffizienten aus der Literatur, da diese bei Gültigkeit des vorgestellten Schemas als eine Kombination von $k_{\mathrm{p}}$ und $k_{\mathrm{p} \text { mid }}$ und somit als „effektive“ Koeffizienten angesehen werden müssen. Eine aussagekräftige Modellierung von SP-PLPSignalen mit diesem Schema ist somit nicht möglich.

Die Aktivierungsenergie für die Übertragungsreaktion ist hoch $(29.3 \mathrm{~kJ} / \mathrm{mol}$ für BA [36]). Deshalb sollten Untersuchungen bei tieferen Temperaturen zu weniger „,mid-chain“ Radikalen und damit geringerem $\alpha$ führen. In Abbildung 8.21 sind die Ergebnisse für Ethylhexylacrylat und Isodecylacrylat bei 1000 bar und verschiedenen Temperaturen dargestellt.

Wie erwartet, ergibt sich für die Messungen bei $10^{\circ} \mathrm{C}$ im Mittel ein geringerer Wert für $\alpha$ als bei $40{ }^{\circ} \mathrm{C}$. Der Unterschied ist jedoch nicht so groß, wie anhand der hohen Aktivierungsenergie für den Übertragungsschritt zu vermuten wäre. Eine Erklärung ergibt sich bei genauerer Betrachtung des vorgestellten kinetischen Schemas.

Es wird davon ausgegangen, dass die „mid-chain“ Radikale nach dem ersten Wachstumsschritt wieder den normalen Radikalen mit aktivem Zentrum am Kettenende zuzurechnen sind. Ein hoher Exponent $\alpha$ resultiert aus einer deutlichen Beteiligung von „midchain“ Radikalen an der Terminierungsreaktion. Eine entsprechend große Konzentration 
dieser Radikale lässt sich durch ein hohes $k_{\text {itr }}$ oder durch eine niedrige Geschwindigkeit für den Wachstumsschritt der ,,mid-chain“ Radikale erreichen.

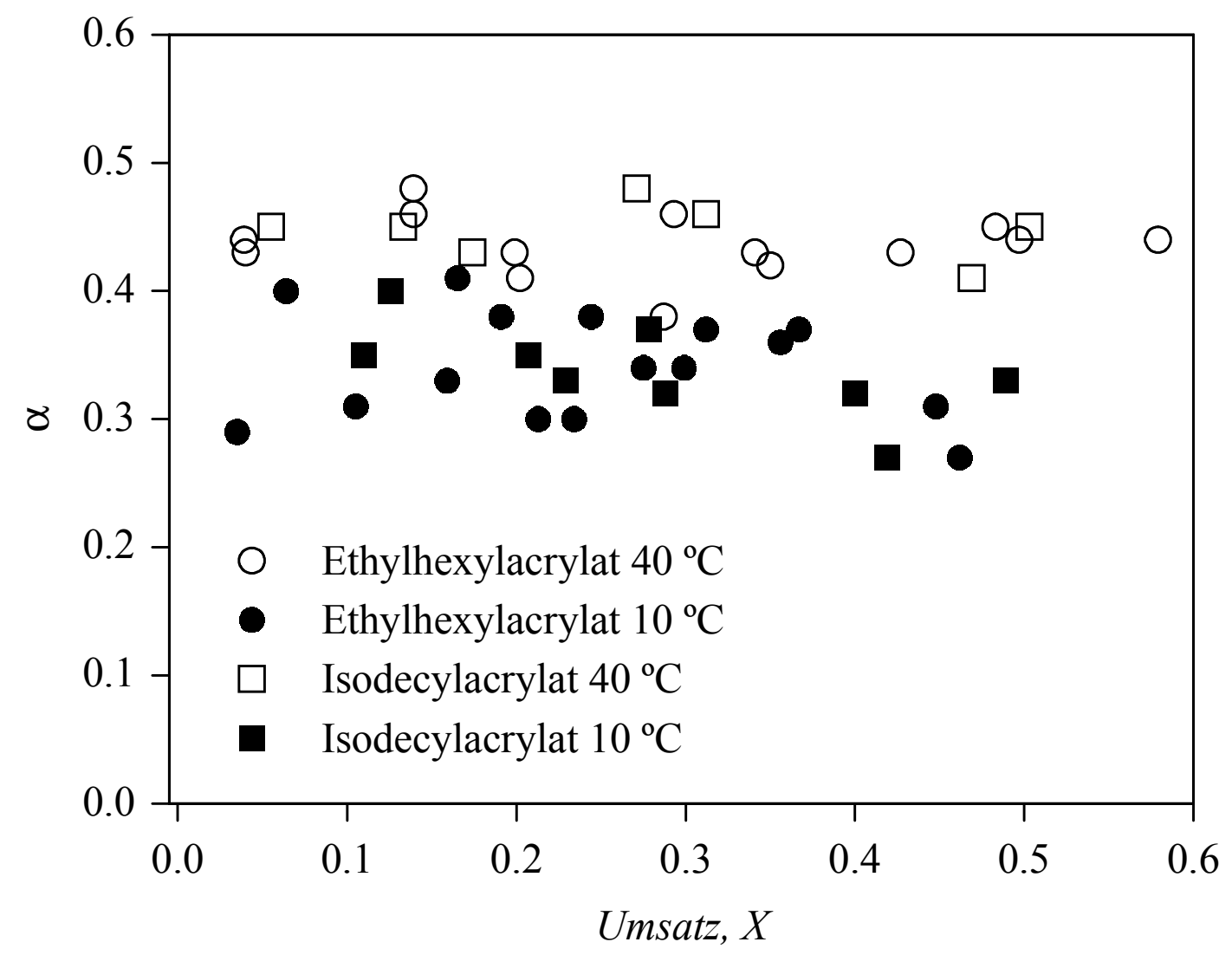

Abb. 8.21: Der Exponent $\alpha$ als Funktion des Monomerumsatzes für Ethylhexylacrylat und Isodecylacrylat bei 1000 bar und verschiedenen Temperaturen.

Als Koeffizient für eine Wachstumsreaktion zeigt auch $k_{\mathrm{p} \text { mid }}$ eine hohe Aktivierungsenergie $[30,37]$. Es werden bei niedrigerer Temperatur zwar weniger „,mid-chain“ Radikale gebildet. Diese werden aber auch langsamer wieder zu ,end-chain“ Radikalen und stehen somit länger für Terminierungsreaktionen zur Verfügung. Bei der Betrachtung des Einflusses von „mid-chain“ Radikalen auf die Kettenlängenabhängigkeit von $k_{\mathrm{t}}$ ist also das Verhältnis $k_{\text {itr }} /\left(k_{\mathrm{p} \text { mid }} \cdot c_{\mathrm{M}}\right)$ entscheidend.

Aus dieser Überlegung heraus ergibt sich aber auch, dass ein Nachweis von verzweigtem Polymer etwa mittels ${ }^{13} \mathrm{C}-\mathrm{NMR}$-Spektroskopie nicht notwendigerweise einen hohen Exponenten $\alpha$ bedeutet. Falls das Wachstum der „mid-chain“ Radikale hinreichend schnell ist, treten verzweigte Ketten auf, obwohl die Terminierungsreaktion nicht durch diese Radikale bestimmt wird. Weitere Hinweise auf den Ursprung der unterschiedlichen 
Kettenlängenabhängigkeit von $k_{\mathrm{t}}$ bei den betrachteten Monomeren können Experimente in Lösung erbringen. Im nächsten Abschnitt werden solche Untersuchungen vorgestellt.

\subsubsection{DA und $\mathrm{BA}$ in überkritischem $\mathrm{CO}_{2}$}

Überkritisches $\mathrm{CO}_{2}\left(\mathrm{scCO}_{2}\right)$ ist als Lösungsmittel für radikalische Polymerisationen aus ökologischen, ökonomischen und praktischen Gründen von großem Interesse. $\mathrm{CO}_{2}$ lässt sich aufgrund des niedrigen kritischen Drucks leicht in den überkritischen Zustandsbereich überführen. Durch Druck- und Temperaturvariation lassen sich die Dichte und Lösungsmittelgüte in einem weiten Bereich verändern, wobei die Viskosität gering bleibt.

Es wurden bereits für zahlreiche Homo- und Copolymerisationen Daten für die Geschwindigkeitskoeffizienten der Wachstums- und Terminierungsreaktion mittels PLP-SEC und SP-PLP in diesem Lösungsmittel ermittelt [38, 39, 40, 41, 42, 43, 44]. Die Verfügbarkeit solcher Daten, die bei ähnlichen Bedingungen wie die in dieser Arbeit vorgestellten Ergebnisse ermittelt wurden, erleichtert die Interpretation der hier erhaltenen Parameter. Aus diesem Grund war für die im Rahmen dieser Arbeit durchgeführten Untersuchungen $\mathrm{CO}_{2}$ anderen Lösungsmitteln vorzuziehen. Bei Experimenten mit $\mathrm{scCO}_{2}$ wird abweichend von der in Kapitel 5 vorgestellten Innenzellentechnik die Hochdruckzelle direkt mit einer Mischung aus Lösungsmittel und Monomer befüllt. Der weitere Ablauf der Messungen entspricht dem für Experimente zur Substanzpolymerisation. Eine Beschreibung der Apparatur zum Befüllen von Hochdruckmesszellen mit Mischungen aus Monomer und überkritischem $\mathrm{CO}_{2}$ findet sich bei Schmaltz [45].

Ziel der Untersuchungen war es, den Einfluss von $\mathrm{scCO}_{2}$ auf die Kettenlängenabhängigkeit von $k_{\mathrm{t}} \mathrm{zu}$ bestimmen. Die Änderungen der Viskosität des Mediums sowie der Lösungsmittelgüte sollten weitere Aufschlüsse darüber geben, welche Mechanismen für die Größe des Parameters $\alpha$ verantwortlich sind. Durch die verringerte Konzentration des Monomers in der Lösung sollten die wachsenden Radikale weniger miteinander verstrickt (,entangled“) vorliegen. In der Abbildung 8.22 sind zunächst die mittleren Terminierungsgeschwindigkeitskoeffizienten $\left\langle k_{\mathrm{t}}>\right.$ für Homopolymerisationen von DA und BA bei $40{ }^{\circ} \mathrm{C} /$ 1000 bar in Substanz und in 40 Gew.\% $\mathrm{CO}_{2}$ gezeigt. 


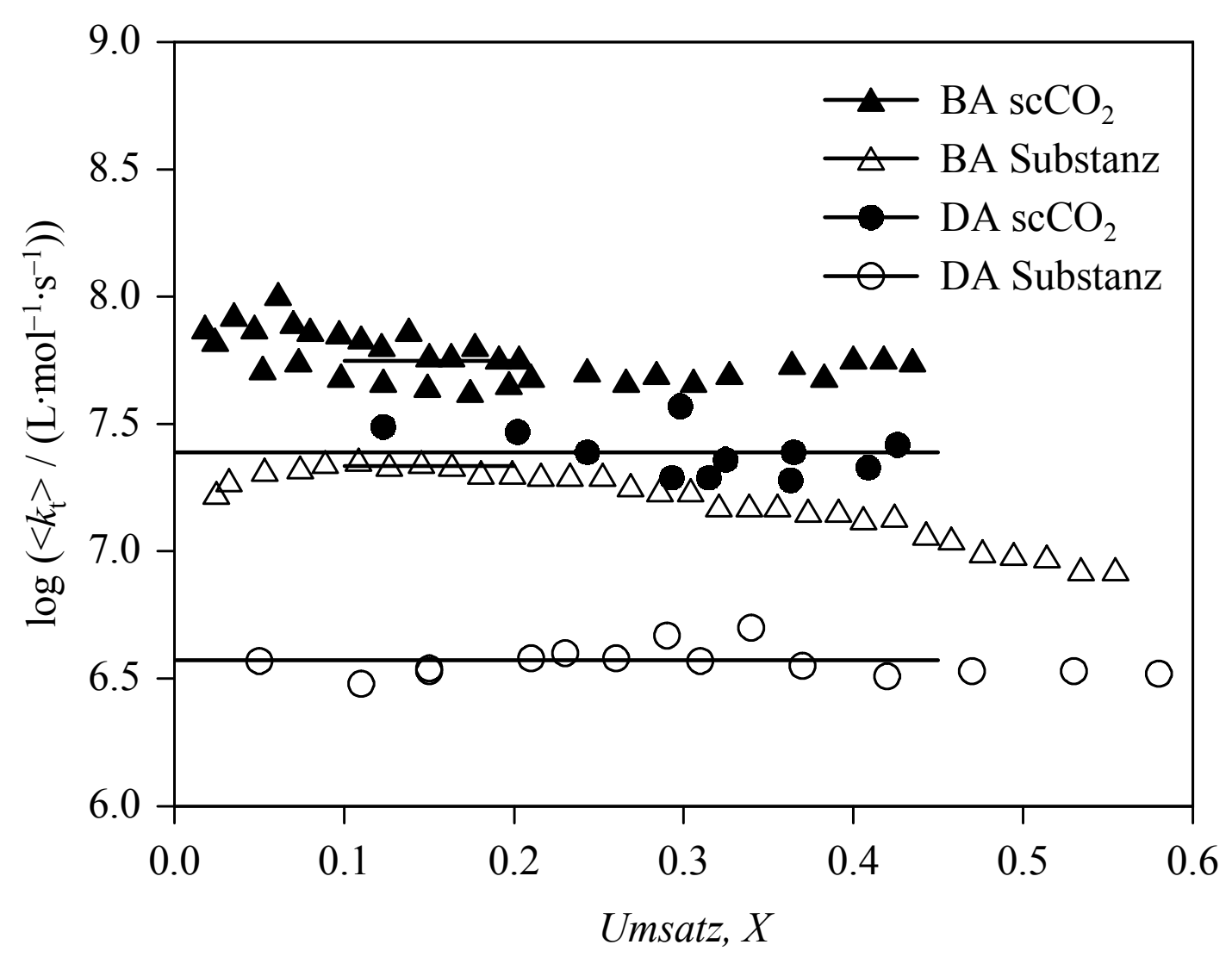

Abb. 8.22: Mittlere Terminierungsgeschwindigkeitskoeffizienten $<k_{\mathrm{t}}>$ für DA und BA bei $40{ }^{\circ} \mathrm{C} / 1000$ bar in Substanz und in 40 Gew. $\% \mathrm{CO}_{2}$.

Der Einfluss von $\mathrm{CO}_{2}$ auf das mittlere $<k_{t}>$ wurde bereits von Nelke [44] mit DMPA als Photoinitiator und längerer Signalaufnahmezeit untersucht. Es wurde festgestellt, dass $<k_{t}>$ bei Zugabe von 40 Gew. \% $\mathrm{CO}_{2}$ um etwa den Faktor 8 erhöht wird. Für die in dieser Arbeit erhaltenen Daten ergibt sich in guter Übereinstimmung für die in Abbildung 8.22 eingezeichneten Mittelwerte eine Steigerung um etwa den Faktor 7. Auch die Absolutwerte von $\left\langle k_{\mathrm{t}}>\right.$ sind denen von Nelke [44] sehr ähnlich. Offensichtlich gleichen sich die Einflüsse durch den nicht idealen Initiator DMPA und die längere Aufnahmezeit aus. Bei der Verwendung von DMPA erhöht sich das gemessene $<k_{t}>$ durch die inhibierende Wirkung eines der Radikalfragmente (siehe Kapitel 6) während durch die Kettenlängenabhängigkeit von $k_{\mathrm{t}}$ das mittlere $<k_{\mathrm{t}}>$ bei längerer Aufnahmezeit abnimmt.

Für die BA-Daten ergibt sich eine befriedigende Übereinstimmung mit den Untersuchungen von Schmaltz [45]. Diese Messungen [45] wurden ebenfalls mit DMPA und längerer Signalaufnahmezeit durchgeführt. Durch die Zugabe von $\mathrm{CO}_{2}$ ergab sich eine Steigerung von $<k_{\mathrm{t}}>$ um etwa den Faktor 4. Bei den Experimenten mit BA fand Schmaltz [45] die Besonderheit, dass $<k_{\mathrm{t}}>\mathrm{zu}$ Beginn der Polymerisation ansteigt und erst nach 10-20\% 
Monomerumsatz in einen Plateaubereich gelangt. Der hier angegebene Faktor für die Zunahme von $<k_{\mathrm{t}}>$ bezieht sich auf diesen Plateaubereich.

Bei den Daten aus Abbildung 8.22 ist ein Ansteigen von $<k_{\mathrm{t}}>$ nur für die BA-Messung in Substanz zu erkennen. Die Messwerte für den Anfangsbereich weisen bei Experimenten in $\mathrm{CO}_{2}$ oftmals eine größere Unsicherheit auf, da geringe Mengen an Sauerstoff in der Messzelle nicht immer völlig zu vermeiden sind und dadurch oftmals eine Inhibierung zu Beginn der Messung zu beobachten ist. So wurden bei den hier diskutierten Messungen in $\mathrm{CO}_{2}$ bei beiden Monomeren zu Beginn des Experiments einige Laserpulse abgegeben, ohne dass eine Reaktion zu beobachten war. Es kann somit nicht ausgeschlossen werden, dass auch bei den ersten „richtigen“ Signalen eine Beeinflussung durch Restsauerstoff vorlag. Diese Inhibierung kann $\mathrm{zu}$ einem höheren gemessenen $<k_{\mathrm{t}}>$ bei niedrigen Umsätzen führen und somit den eigentlich zu beobachtenden Anstieg dieses Koeffizienten ausgleichen bzw. unsichtbar werden lassen. Für den bei der Messung in Substanz gefundenen Plateaubereich zwischen 10 und $20 \%$ Umsatz ergibt sich in guter Übereinstimmung mit den Daten von Schmaltz [45] eine Zunahme von $<k_{\mathrm{t}}>$ um etwa den Faktor 3.

In Abbildung 8.23 zeigt den Parameter $\alpha$ für DA in Substanz und in $\mathrm{CO}_{2}$ aus einer Auswertung der schon in Abbildung 8.22 vorgestellten Messungen unter Berücksichtigung der Kettenlängenabhängigkeit von $k_{\mathrm{t}}$.

Innerhalb der Messungenauigkeit ist für DA kein Einfluss des $\mathrm{CO}_{2}$ auf den Exponenten $\alpha \mathrm{zu}$ erkennen, d. h. auch in Lösung ergibt sich ein $\alpha$ von etwa 0.4. Die Werte für Messungen mit $\mathrm{CO}_{2}$ weisen eine etwas größere Streuung auf, was sich durch die schlechtere Signalqualität erklären lässt. Das liegt einerseits an der durch das Lösungsmittel verringerten Monomerkonzentration und andererseits an der bereits beschriebenen Zunahme des mittleren $<k_{\mathrm{t}}>$ bei den Messungen in $\mathrm{CO}_{2}$, was sich weiterhin verschlechternd auf das Signal-RauschVerhältnis auswirkt. 


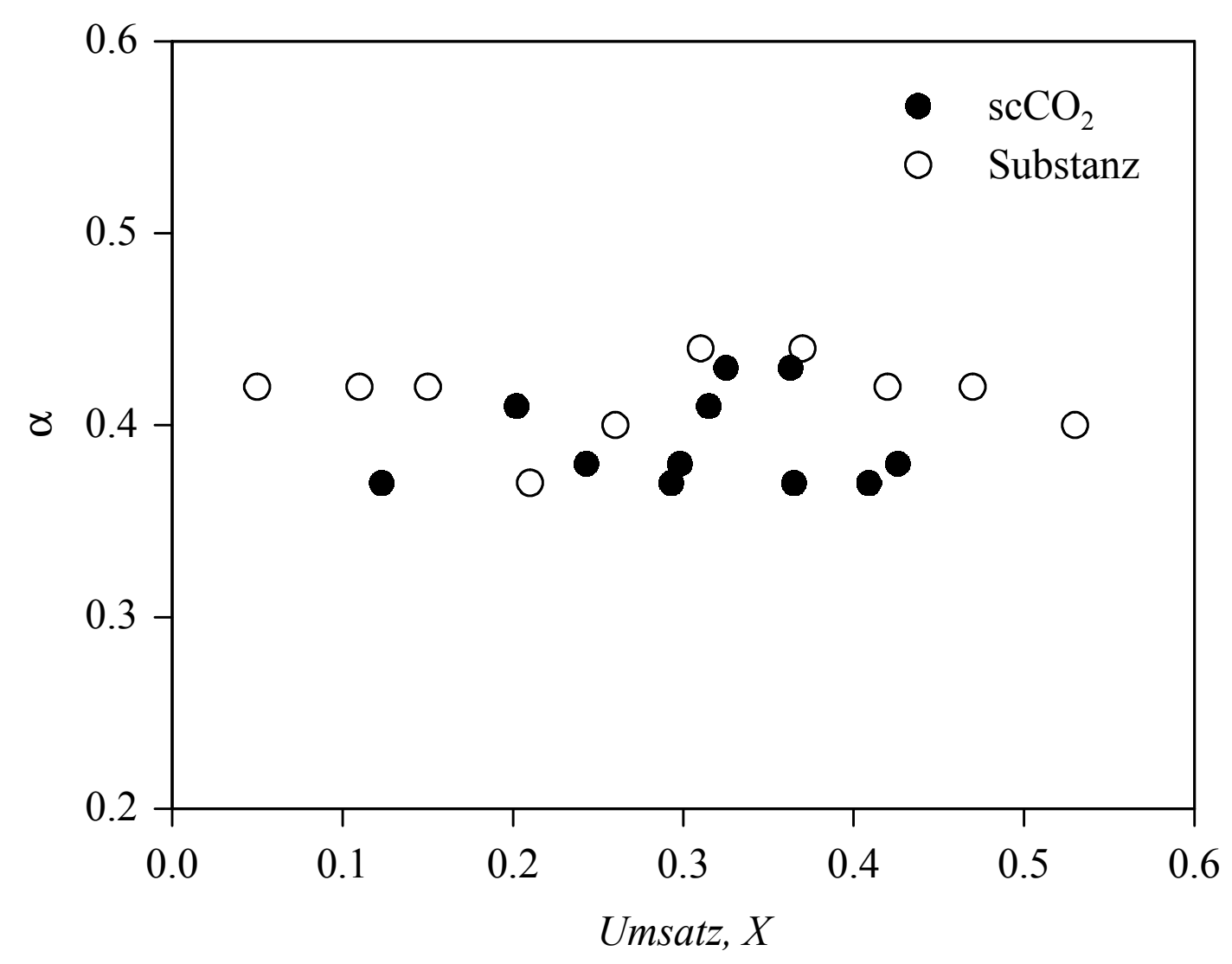

Abb. 8.23: Der Exponent $\alpha$ als Funktion des Monomerumsatzes für die Homopolymerisation von DA in Substanz und in 40 Gew. $\% \mathrm{CO}_{2}$ bei $40{ }^{\circ} \mathrm{C} / 1000$ bar.

Überkritisches $\mathrm{CO}_{2}$ ist im Vergleich zu Acrylaten mit kurzkettigen Estergruppen für DA und Poly-DA ein gutes Lösungsmittel. Die Viskosität der Mischung erniedrigt sich durch die Zugabe von $\mathrm{CO}_{2}$. Dadurch erhöht sich die Beweglichkeit der Radikale und sie überlappen häufiger miteinander. Somit sollte $\mathrm{CO}_{2} \mathrm{zu}$ einer Erhöhung von $k_{\mathrm{t}}^{0}$ und damit $\mathrm{zu}$ der schon erwähnten Steigerung des mittleren $<k_{t}>$ ohne Zunahme von $\alpha$ führen. In Abbildung 8.24 ist $k_{\mathrm{t}}^{0}$ für DA und BA jeweils in Substanz und in $\mathrm{CO}_{2}$ bei $40^{\circ} \mathrm{C} / 1000$ bar dargestellt.

Im Fall von DA in $\mathrm{CO}_{2}$ zeigt sich ab etwa $20 \%$ Monomerumsatz ein nahezu konstantes $k_{\mathrm{t}}{ }^{0}$. Unter $20 \%$ Umsatz sind die Daten wegen der bereits erwähnten möglichen Inhibierung durch Restsauerstoff mit einem größeren Fehler behaftet. Im konstanten Bereich liegen die Werte für $k_{\mathrm{t}}^{0}$ bei den Messungen mit $\mathrm{CO}_{2}$ deutlich über denen in Substanz. Der Unterschied beträgt etwa den Faktor 4, was noch nicht die bei $<k_{t}>$ gefundene Differenz (Faktor 7) erklärt. Jedoch sind beim mittleren $\left\langle k_{\mathrm{t}}\right\rangle$ auch Einflüsse durch die Signalaufnahmezeit und damit durch die Kettenlänge zu berücksichtigen. So ist bei gleicher Aufnahmezeit wegen der geringeren Monomerkonzentration in $\mathrm{CO}_{2}$ die mittlere Kettenlänge kleiner und damit $<k_{\mathrm{t}}>$ größer. 


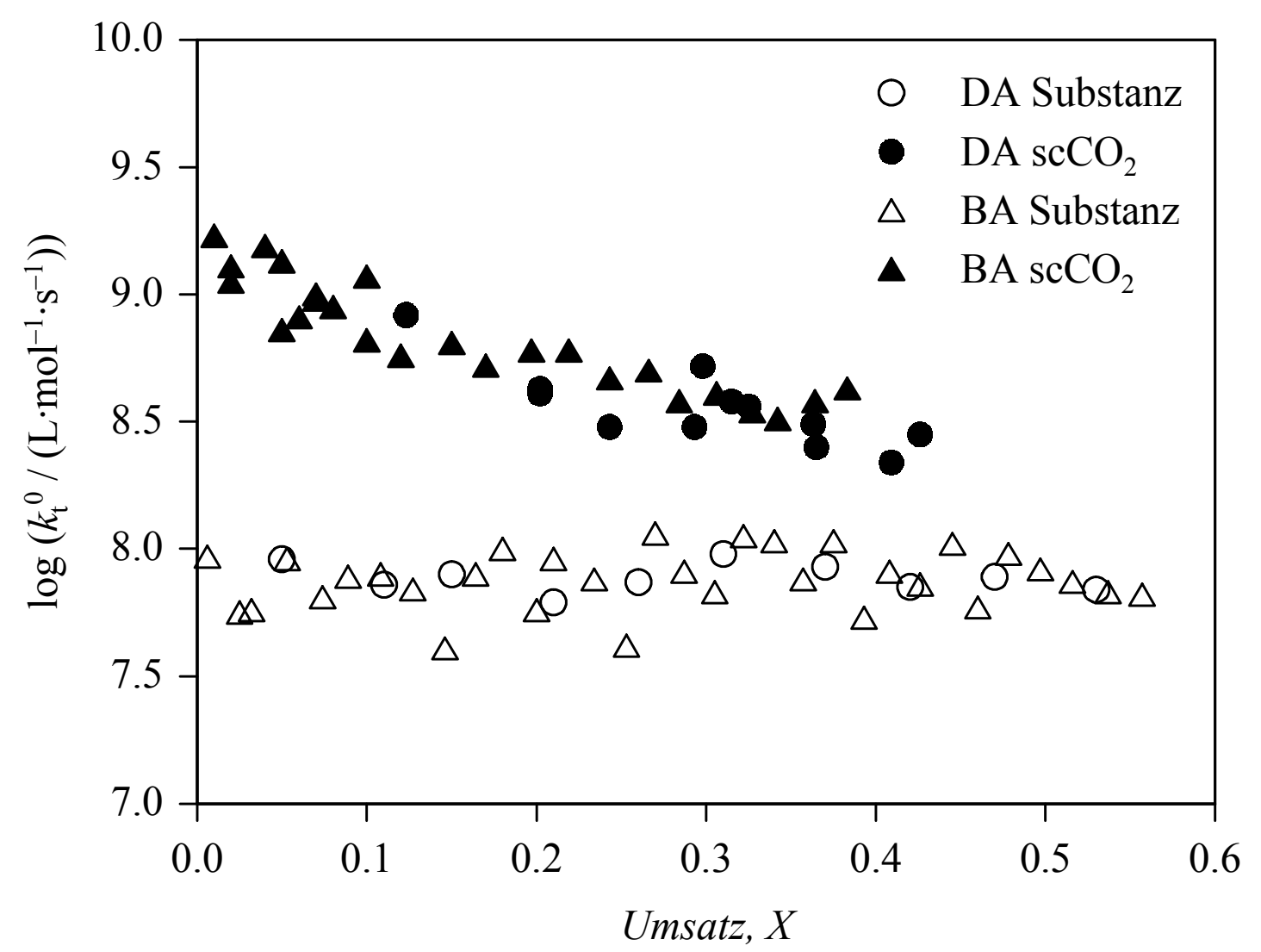

Abb. 8.24: Umsatzabhängigkeit von $\log \left(k_{\mathrm{t}}^{0} /\left(\mathrm{L} \cdot \mathrm{mol}^{-1} \cdot \mathrm{s}^{-1}\right)\right)$ für Homopolymerisationen von DA und BA in Substanz sowie in 40 Gew. $\% \mathrm{CO}_{2}$ bei $40{ }^{\circ} \mathrm{C} / 1000$ bar.

Bei BA in $\mathrm{CO}_{2}$ im Anfangsbereich bis etwa $10 \%$ Umsatz als Auffälligkeit ein abfallendes $k_{\mathrm{t}}{ }^{0}$ zu beobachten. Bei der Diskussion der Daten aus Abbildung 8.22 wurde bereits erwähnt, dass für das mittlere $\left\langle k_{\mathrm{t}}>\right.$ zu Beginn der Polymerisation ungewöhnlich hohe Werte erhalten wurden. Dieser Befund zeigt sich nun auch im $k_{\mathrm{t}}^{0}$. Bei den Untersuchungen in $\mathrm{CO}_{2}$ ergibt sich also sowohl für DA als auch für BA bei niedrigen Monomerumsätzen die Besonderheit eines sehr hohen $k_{\mathrm{t}}^{0}$. Erklären lässt sich dies durch Reste von Sauerstoff oder einer anderen Verunreinigung, die zu Beginn der Polymerisation zu verstärkter Terminierung führt.

Die Werte für $k_{\mathrm{t}}^{0}$ von BA in Substanz und in $\mathrm{CO}_{2}$ unterscheiden sich zwischen 10 und $20 \%$ Monomerumsatz um den Faktor 10. In diesem Bereich zeigte sich für $\left\langle k_{t}\right\rangle$ in Substanz ein Plateau (Abbildung 8.22) und es wurde ein Steigerung von $<k_{\mathrm{t}}>$ durch $\mathrm{CO}_{2}$-Zugabe um den Faktor 4 festgestellt. Durch das Lösungsmittel $\mathrm{CO}_{2}$ erhöht sich also im Fall von BA $k_{\mathrm{t}}^{0}$ deutlich stärker als $\left\langle k_{\mathrm{t}}>\right.$. Dieser Unterschied ist größer als bei DA und es kann davon ausgegangen werden, dass bei BA auch der Exponent $\alpha$ durch das $\mathrm{CO}_{2}$ beeinflusst (d. h. erhöht) wird. In Abbildung 8.25 ist $\alpha$ für Homopolymerisationen von BA in Substanz und in $\mathrm{scCO}_{2}$ bei $40{ }^{\circ} \mathrm{C} / 1000$ bar dargestellt. 
Für BA ergibt sich wie vermutet ein völlig anderes Bild für den Einfluss des $\mathrm{CO}_{2}$ auf $\alpha$ als bei DA. Wie in Abbildung 8.25 gezeigt, erhöht sich $\alpha$ bei niedrigen Monomerumsätzen von etwa 0.15 in Substanz auf etwa 0.35 in Lösung. Außerdem steigt der Exponent ohne Lösungsmittel mit dem Monomerumsatz bis auf etwa 0.3 an, so dass sich die Werte für $\alpha$ bei höheren Umsätzen annähern. In $\mathrm{CO}_{2}$ scheint $\alpha$ zu höheren Umsätzen hin etwas abzunehmen. Dafür kann aber kein physikalischer Grund gefunden werden. Im Anfangsbereich bis $10 \%$ Umsatz wurden auffällig hohe Werte für $k_{\mathrm{t}}^{0}$ ermittelt (Abbildung 8.24). Aufgrund der bereits beschriebenen Korrelation von $k_{\mathrm{t}}^{0}$ und $\alpha$ ist es denkbar, dass auch die Werte von $\alpha$ zu Beginn der Polymerisation etwas überschätzt werden und eigentlich im Bereich der zwischen 20 und $40 \%$ Umsatz erhaltenen Daten liegen müssten. Trotz dieser Unsicherheit zu Beginn der Polymerisation unterscheiden sich die Ergebnisse für $\alpha$ in Substanz und in $\mathrm{CO}_{2}$ deutlich.

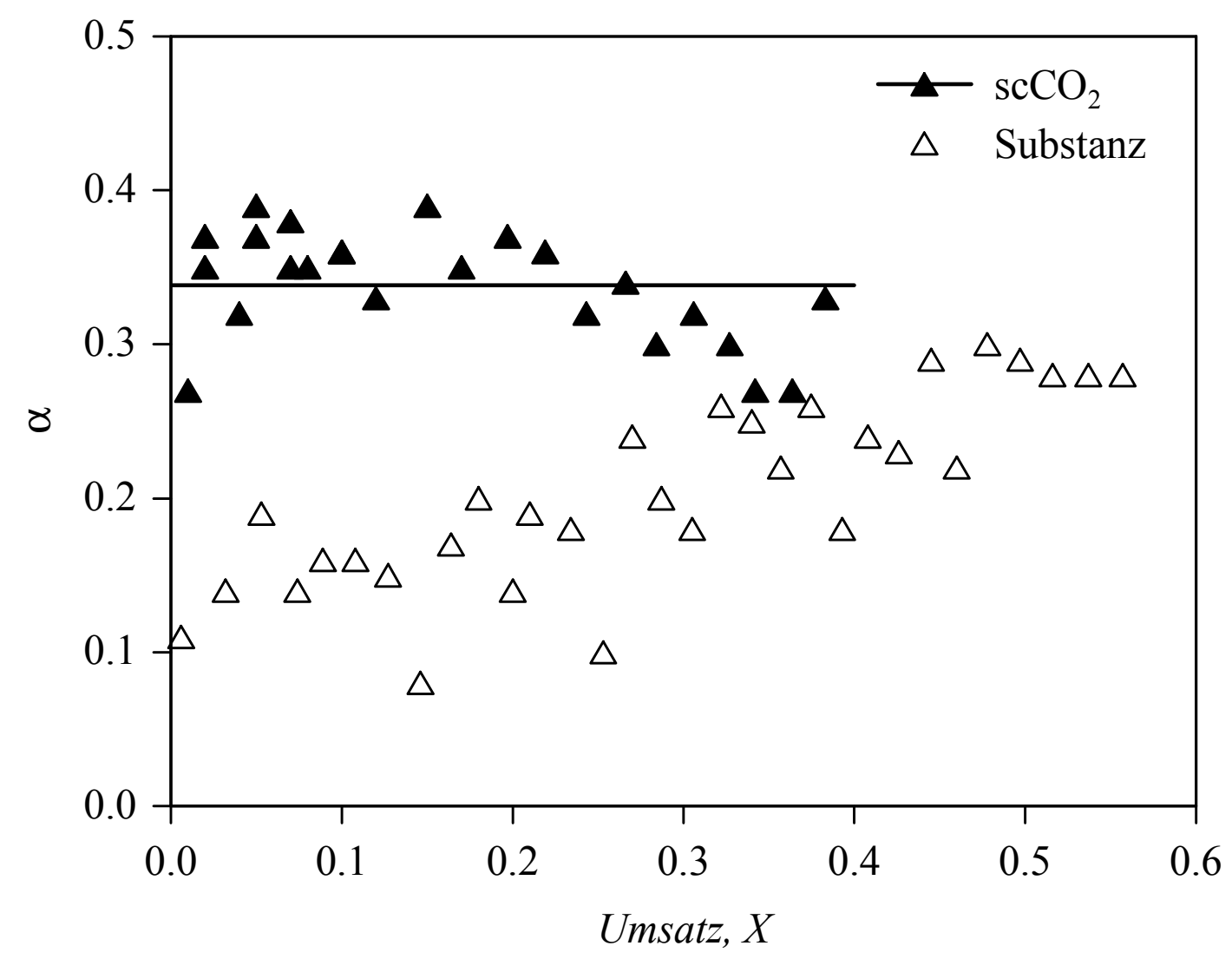

Abb. 8.25: Der Exponent $\alpha$ als Funktion des Monomerumsatzes für die Homopolymerisation von BA in Substanz und in 40 Gew. $\% \mathrm{CO}_{2}$ bei $40{ }^{\circ} \mathrm{C} / 1000$ bar.

Für Poly-BA ist $\mathrm{CO}_{2}$ ein schlechteres Lösungsmittel als für Poly-DA und es kann davon ausgegangen werden, dass es bei den Makroradikalen zu einer verstärkten Knäuelbildung kommt. Dadurch wird die Wahrscheinlichkeit einer intramolekularen Übertragungsreaktion 
mit der Bildung von „,mid-chain“ Radikalen erhöht. Dieser Effekt tritt vor allem in schlechten Lösungsmitteln auf.

In der Literatur finden sich einige Beispiele dafür, dass im Allgemeinen durch die Zugabe eines Lösungsmittels während der Reaktion der Anteil an verzweigtem Polymer zunimmt $[28,30,31,32,36]$. Bei der Diskussion zur Temperaturabhängigkeit von $\alpha$ wurde bereits erwähnt, dass bei der Betrachtung des Einflusses von „mid-chain“ Radikalen auf die Terminierungsreaktion das Verhältnis $k_{\text {itr }} /\left(k_{\mathrm{p} \text { mid }} \cdot c_{\mathrm{M}}\right)$ entscheidend ist. Durch die Verringerung der Monomerkonzentration wird somit die Zeit für den Wachstumsschritt dieser Radikale und damit ihre Lebensdauer sowie Konzentration erhöht.

Im Rahmen dieser Argumentation ist auch der Anstieg von $\alpha$ mit dem Umsatz bei einigen oben vorgestellten Monomeren in Substanz verständlich. Auch in diesen Fällen äußert sich die Abnahme der Monomerkonzentration in einer Zunahme des Verhältnisses von „,midchain“ zu „end-chain“ Radikalen. Eine Zunahme des Anteils von verzweigtem Polymer mit dem Umsatz wurde bereits von Azukizawa et al. [35] für Phenylacrylat berichtet.

Für die hier diskutierte Modellvorstellung ist ein Wert von $\alpha=0.43$ als Grenzwert für eine durch „mid-chain“ Radikale dominierte Terminierungsreaktion zu verstehen. Im Fall von DA kann zwar nicht ausgeschlossen werden, dass durch das $\mathrm{CO}_{2}$ als Lösungsmittel die Konzentration an „mid-chain“ Radikalen erhöht wird. Da jedoch der Grenzwert von $\alpha$ bereits erreicht ist, wirkt sich eine weitere Steigerung der Konzentration dieser Radikalen nicht mehr auf das Ausmaß der Kettenlängenabhängigkeit von $k_{\mathrm{t}}$ aus.

Die Werte für $k_{\mathrm{t}}^{0}$ bei den Polymerisationen in Substanz (Abbildung 8.24) unterscheiden sich für BA und DA nicht. Die Differenz im mittleren $<k_{t}>$ für diese beiden Monomere von fast einer Größenordnung [45, 46] (vgl. Abbildung 8.22) ist somit auf die stark unterschiedliche Kettenlängenabhängigkeit des Terminierungsgeschwindigkeitskoeffizienten zurückzuführen. Im Gegensatz zu dem bereits diskutierten Vergleich von DMA und DA ist bei BA/DA die Betrachtung des mittleren $<k_{\mathrm{t}}>$ bei gleicher Signalaufnahmezeit (wie in der Abbildung 8.22 dargestellt) durchaus gerechtfertigt. Die Literaturdaten für $k_{\mathrm{p}}$ sind bei $n$-Acrylaten recht ähnlich $[45,46]$, so dass bei der Analyse von DA und BA hinsichtlich $\left\langle k_{t}>\right.$ gleiche Zeitintervalle auch annähernd gleiche Kettenlängen bedeuten. 


\subsection{6 $\alpha$ für MA-DA Copolymerisationen}

Bei Kenntnis der Kettenlängenabhängigkeit von $k_{\mathrm{t}}$ für viele Monomere erschien es interessant, das Verhalten von $\alpha$ bei Copolymerisationen zu untersuchen. Als Copolymerisationssystem wurde MA-DA ausgewählt, weil hier mit einer guten Signalqualität zu rechnen ist und große Unterschiede für $\alpha$ bei den entsprechenden Homopolymerisationen gefunden wurden. In Abbildung 8.26 sind die Ergebnisse für eine MA-DA Copolymerisation mit gleichen Molenbrüchen für beide Monomere $\left(f_{\mathrm{MA}}=f_{\mathrm{DA}}=0.5\right)$ sowie für die entsprechenden Homopolymerisationen bei $40{ }^{\circ} \mathrm{C} / 1000$ bar dargestellt.

Wie aus der Abbildung zu erkennen ist, ergibt sich der Parameter $\alpha$ nahezu als der Mittelwert der Werte für die Homopolymerisationen.

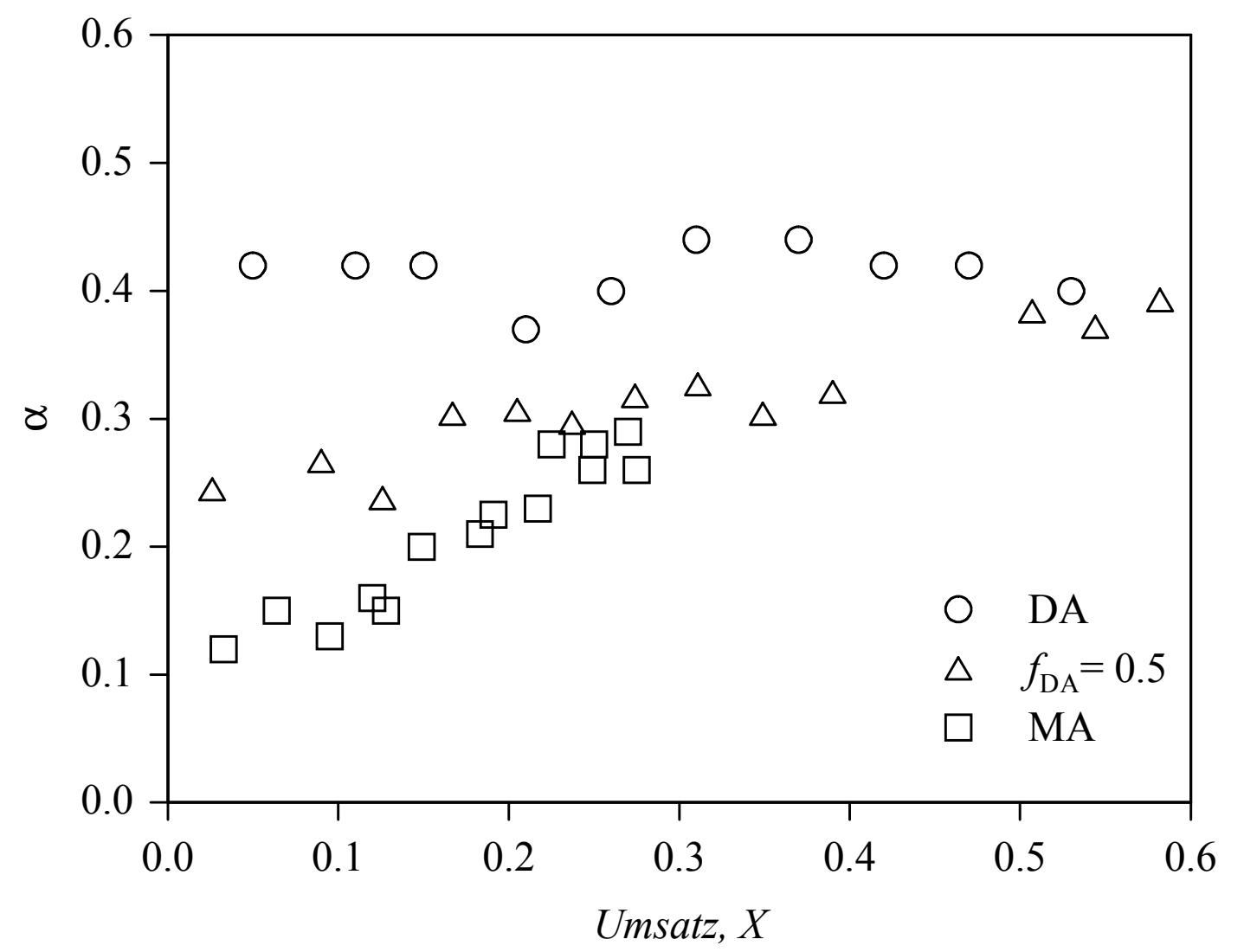

Abb. 8.26: Der Exponent $\alpha$ als Funktion des Monomerumsatzes für Homopolymerisationen von DA und MA sowie einer Copolymerisation mit gleichen Molenbrüchen beider Monomere bei $40{ }^{\circ} \mathrm{C} / 1000$ bar.

Für Acrylat-Acrylat Copolymerisationen ist bekannt [47], dass beide Monomere mit gleicher Wahrscheinlichkeit in das Polymer eingebaut werden, was sich durch die sehr ähnlichen 
Copolymerisationsparamter $r_{\mathrm{i}}$ (vgl. Kapitel 7) ausdrückt. Wenn also die Anzahl der für eine Transferreaktion zur Verfügung stehenden Wasserstoffatome und die Wahrscheinlichkeit der Abstraktion für das Ausmaß der Bildung von „mid-chain“ Radikalen verantwortlich ist, dann sollte sich $\alpha$ bei Copolymerisationen entsprechend den Anteilen der Monomere im Copolymer verhalten.

Es sei an dieser Stelle noch erwähnt, dass für die hier verwendete Auswertung gemäß Gleichung 8.8 die Zeit für einen Wachstumsschritt $t_{\mathrm{p}}=\left(k_{\mathrm{p}} \cdot c_{\mathrm{M}}\right)^{-1}$ sowie für die Bestimmung von $k_{\mathrm{t}}^{0}$ aus dem Fitparameter $\mathrm{c} \equiv 2 \cdot k_{\mathrm{t}}^{0} / k_{\mathrm{p}}$ der Wachstumsgeschwindigkeitskoeffizient $k_{\mathrm{p}}$ bekannt sein muss. Dabei ergeben sich für Copolymerisationen mit stark unterschiedlichen Copolymerisationsparametern, wie es etwa bei den Methacrylat-Acrylat Copolymerisationen der Fall ist [48, 49], einige Probleme. Bei diesen Systemen ändert sich die Zusammensetzung der Monomermischung mit dem Umsatz und damit auch $k_{\mathrm{p}}$ [50]. Es muss also mit der in Kapitel 7 beschriebenen Prozedur für jeden Umsatz die aktuelle Zusammensetzung errechnet werden, was sich als zusätzlicher Fehler auf die Ergebnisse auswirkt. Bei dem in diesem Kapitel untersuchten feinen Effekt einer Kettenlängenabhängigkeit von $k_{\mathrm{t}}$ und der bei Methacrylaten (bzw. Copolymerisationen mit hohem Methacrylat-Anteil) ohnehin schlechteren Signalqualität ist aus diesem Grund die Untersuchung von Methacrylat-Acrylat Systemen sehr schwierig.

\subsection{Schlussfolgerungen}

Die Untersuchungen in diesem Kapitel haben gezeigt, dass mit dem Photoinitiator MMMP die modellabhängige Bestimmung der Kettenlängenabhängigkeit von $k_{\mathrm{t}}$ aus einzelnen Messsignalen möglich ist. Eine Unterscheidung zwischen verschiedenen Modellen, die eine Abnahme von $k_{\mathrm{t}}$ mit der Kettenlänge beschreiben, gelingt nicht. Es werden jedoch für das verwendete Potenzgesetz von der Fitprozedur unabhängige Ergebnisse erhalten.

Eine verbesserte Genauigkeit ließe sich vermutlich über eine unabhängige Bestimmung eines der Koeffizienten erreichen. So kann durch Zugabe eines Transferagens die Kettenlänge der Radikale variiert werden. Eine Auswertung entsprechender SP-PLP-Signale nach idealer Polymerisationskinetik würde ein $<k_{t}>$ für eine mittlere, von der Konzentration des Transferagens abhängige Kettenlänge ergeben. Dabei muss auf eine korrekte Mittelung der Kettenlängen aus den SEC-Daten geachtet werden. Die Ergebnisse könnten bis auf sehr kleine Kettenlängen extrapoliert und mit dem in dieser Arbeit erhaltenen $k_{t}^{0}$ verglichen werden. Erste Untersuchungen mit dieser Methode wurden bereits durchgeführt [51]. Einen alternativen 
Zugang zu $k_{\mathrm{t}}^{0}$ bietet die in Kapitel 8.3.1 erwähnte Methode von Vana et al. [19]. Dort wird ausgenutzt, dass mittels spezieller Polymerisationstechniken („,kontrollierte/ lebende radikalische Polymerisation") auch unter stationären Bedingungen ein linearer Zusammenhang zwischen Zeit und Kettenlänge erreicht werden kann. Die kinetische Analyse bei kurzen Zeiten erlaubt dann die Bestimmung von $k_{\mathrm{t}}$ für sehr kleine Radikale.

Mit der in dieser Arbeit verwendeten Methode ergibt sich ein Parameter $\alpha$, der für die untersuchten Methacrylate um 0.15 bei niedrigen Umsätzen bestimmt wird und dann mit dem Umsatz ansteigt. Der Umsatzverlauf zeigt, dass bei höheren Umsätzen andere Diffusionsprozesse für $k_{\mathrm{t}}$ bestimmend sind als zu Beginn der Polymerisation.

Bei Acrylaten sind aufgrund der größeren Reaktivität und der höheren Flexibilität der Makroradikale mehr Nebenreaktionen möglich. Für diese Monomere ergibt sich eine Zunahme von $\alpha$ mit der Anzahl von abstrahierbaren Wasserstoffatomen im Esterrest bzw. der Stabilität der durch Abstraktion gebildeten Radikale und dem Monomerumsatz, wobei maximal ein Wert um 0.43 erreicht wird. Die Ergebnisse deuten darauf hin, dass bei den Acrylaten mit großer Seitenkette ein hoher Anteil von wachsenden Spezies mit der Radikalfunktion in der Mitte der Kette (,,mid-chain“ Radikale) für den großen Wert von $\alpha$ verantwortlich ist. Diese Radikale werden durch Übertragungsreaktionen gebildet, deren relative Wahrscheinlichkeit mit dem Umsatz zunimmt. Bei Monomeren mit wenigen zur Verfügung stehenden H-Atomen kann dies einen Anstieg von $\alpha$ zur Folge haben.

Die Theorie zur Größe der Kettenlängenabhängigkeit von $k_{\mathrm{t}}[23,24]$ ist für unverzweigte Makroradikale in verdünnter Lösung entwickelt worden. Die hier vorgestellten Experimente wurden aber im Wesentlichen in halbverdünnter oder konzentrierter Lösung durchgeführt. Außerdem führt ein großer Anteil an „mid-chain“ Radikalen zur Bildung von verzweigten Ketten. Ob die Vorhersagen für lineare Ketten auch für Systeme gültig sind, in denen sowohl die wachsenden Radikale als auch das tote Polymermoleküle verzweigt sind, muss noch durch weitere experimentelle Untersuchungen und theoretische Betrachtungen geklärt werden.

Das Auftreten von „mid-chain“ Radikalen bei der radikalischen Polymerisation hat weitreichende Konsequenzen für die Bestimmung von kinetischen Koeffizienten. So wurde von Yamada und Mitarbeitern eine geringere Aktivität dieser Radikale diskutiert [33, 35]. Daraus ergibt sich eine Erschwernis bei der Bestimmung des Wachstumsgeschwindigkeitskoeffizienten $k_{\mathrm{p}}$ mit der PLP-SEC-Technik. Es wurde vermutet [33, 35], dass die Spezies mit dem aktiven Zentrum in der Mitte der Kette langsamer mit den durch die Laserpulse neu gebildeten Primärradikalen terminieren. Auf diese Weise überlebt eine größere Konzentration 
dieser Radikale mehrere Laserpulse. Diese Population kann also länger wachsen und die Polymermolmasse an dem für die PLP-SEC-Technik wichtigen ersten Wendepunkt der Molmassenverteilung wird größer im Vergleich zum ideal verlaufenden Experiment. Aus diesen Überlegungen folgt also, dass $k_{\mathrm{p}}$ mit PLP-SEC überschätzt wird. Andererseits müssen die gemessenen $k_{\mathrm{p}}$-Werte als „effektive“ Koeffizienten aus einer Kombination von $k_{\mathrm{p}}$ und $k_{\mathrm{p} \text { mid }}$ angesehen werden. Die Qualität der Bestimmung von $k_{\mathrm{p}}$ wirkt sich direkt auf die Ermittlung von $k_{\mathrm{t}}$ und $k_{\mathrm{t}}^{0}$ aus den gekoppelten Parametern $k_{\mathrm{t}} / k_{\mathrm{p}}$ bzw. $k_{\mathrm{t}}^{0} / k_{\mathrm{p}}$ aus. Für eine genaue Bestimmung von $k_{\mathrm{p}}$ mit PLP-SEC wäre die Analyse von sehr kurzen Ketten, bei denen noch kein Transferschritt stattgefunden hat, notwendig. Eine andere Möglichkeit ist die Durchführung der Experimente bei möglichst niedriger Temperatur, so dass die Bildung der „mid-chain“ Radikale wegen der hohen Aktivierungsenergie der entsprechenden Reaktion [37] weitgehend vermieden wird. Die in dieser Arbeit zur Berechnung von $<k_{\mathrm{t}}>$ bzw. $k_{\mathrm{t}}^{0}$ verwendeten $k_{\mathrm{p}}$-Werte für die Acrylate [47] sind von niedrigerer Temperatur auf $40{ }^{\circ} \mathrm{C}$ extrapolierte Koeffizienten. Wenn bei höherer Temperatur vermehrt „mid-chain“ Radikale auftreten mit Wachstumsgeschwindigkeitskoeffizienten, die sich von denen der „end-chain“ Radikalen unterscheiden, so ist diese Extrapolation als zusätzliche Fehlerquelle in den Experimenten zu beachten.

Die in diesem Kapitel diskutierte Kettenlängenabhängigkeit von $k_{\mathrm{t}}$ muss auch beim Vergleich verschiedener Monomere bezüglich ihrer mittleren Terminierungsgeschwindigkeitskoeffizienten $<k_{t}>$ berücksichtigt werden. Insofern stellt die auf Seite 36 gezeigt „Landkarte“ (Abbildung 6.1) einiger Acrylate und Methacrylate bezüglich $k_{\mathrm{t}}$ eine grobe Näherung dar. Es ist darauf zu achten, dass für die betrachteten Monomere das mittlere $k_{\mathrm{t}}$ für die gleiche Kettenlänge angegeben wird. Besonders bei SP-PLP-Experimenten ist also der lineare Zusammenhang zwischen Signalaufnahmezeit und betrachteter Kettenlänge mit einzubeziehen. Dies hat wegen der deutlich unterschiedlichen $k_{\mathrm{p}}$-Werte vor allem Auswirkungen beim Vergleich von Methacrylaten mit Acrylaten und wird offensichtlich bei der Gegenüberstellung von DMA und DA, wo bei gleicher Signalaufnahmezeit ein ähnliches $<k_{\mathrm{t}}>$ für beide Monomere resultiert, während die Analyse gleicher Kettenlängen einen klaren Unterschied für diesen Koeffizienten ergibt. Insofern mag eine Betrachtung von $k_{\mathrm{t}}^{0}$ anstatt $<k_{\mathrm{t}}>$ beim Vergleich verschiedener Monomere als einfache Alternative erscheinen. Dabei ist aber zu beachten, dass $k_{\mathrm{t}}^{0}$ nur formal die Terminierungsreaktion zwischen zwei sehr kleinen Radikalen beschreibt und außerdem mit $\alpha$ korreliert ist. Der extrapolierte Wert kann sich durchaus vom mit der hier verwendeten Technik experimentell nicht zugänglichen $k_{\mathrm{t}}$ für 
Radikale der Kettenlänge eins unterscheiden. Beim Vergleich der Abfolgen von $k_{\mathrm{t}}^{0} \mathrm{bzw}$. von $<k_{\mathrm{t}}>$ innerhalb der Acrylate fällt auf, dass für BA und DA nahezu gleiche $k_{\mathrm{t}}^{0}$-Werte erhalten werden, während $<k_{\mathrm{t}}>$ bei DA um etwa eine Größenordung niedriger als bei BA ist. Diese Diskrepanz tritt also gerade bei zwei Monomeren auf, von denen für dass eine (DA) ein signifikanter Einfluss von „mid-chain“ Radikalen auf die Polymerisation vermutet werden kann, während beim anderen (BA) die Konzentration dieser Radikale deutlich geringer ist. Beim Vergleich von BA mit MA wird diese Auffälligkeit nicht beobachtet. Ein Einfluss von $k_{\mathrm{p}}$ kann ausgeschlossen werden, da bei der Bestimmung von $k_{\mathrm{t}}^{0}$ und $<k_{\mathrm{t}}>$ aus den gekoppelten Parametern $k_{\mathrm{t}} / k_{\mathrm{p}}$ bzw. $k_{\mathrm{t}}^{0} / k_{\mathrm{p}}$ dieselben Werte für $k_{\mathrm{p}}$ verwendet wurden. Als Ursache für den vergleichsweise erhöhten $k_{\mathrm{t}}^{0}$-Wert von DA kommt die Korrelation zwischen $k_{\mathrm{t}}^{0}$ und $\alpha$ infrage. So ergibt sich auch bei den anderen Monomeren mit hohem $\alpha$ ein ähnlicher Wert für $k_{\mathrm{t}}^{0}$ wie bei DA. Ebenso ist im Fall von BA in $\mathrm{scCO}_{2}$ eine Zunahme beider Koeffizienten zu beobachten.

Andererseits kann auch argumentiert werden, dass die Größe der Esterseitenkette nur einen geringen Einfluss auf $k_{\mathrm{t}}^{0}$ hat. Dann wäre nicht die Ähnlichkeit bei DA und BA auffällig, sondern der besonders hohe Wert für MA. Im Rahmen dieser Argumentation kann vermutet werden, dass bei MA nur eine geringe Abschirmung der Radikalfunktion durch die Seitenkette stattfindet und so die Terminierungsreaktion nur wenig behindert wird. Ab einer gewissen Größe des Esterrests (d.h. ab der Butylgruppe im BA) wirkt sich eine weitere Zunahme der Abschirmung nur noch geringfügig aus. Die Unterschiede im mittleren $<k_{\mathrm{t}}>$ zwischen verschiedenen Monomeren sind dann nur in der Größe der Kettenlängenabhängigkeit begründet. Zur Klärung dieser Effekte wäre die unabhängige Bestimmung von $k_{\mathrm{t}}^{0}$ wünschenswert.

Im Fall von Copolymerisationen muss ebenfalls bei Variation der Monomermischung auf die Kettenlänge geachtet werden. Dazu können entsprechend der in Kapitel 7 vorgestellten Prozedur die aktuellen Mischungszusammensetzungen bei fortschreitendem Umsatz und damit die korrekten Wachstumsgeschwindigkeitskoeffizienten berechnet werden, woraus sich schließlich die optimale Signalaufnahmezeit bestimmen lässt.

Die Modellierung von $k_{\mathrm{t}}$ bei Copolymerisationen stellt sich mit einer für die beiden Monomere unterschiedlichen Kettenlängenabhängigkeit von $k_{\mathrm{t}}$ völlig neu dar. Der Koeffizient $k_{\mathrm{t}, \mathrm{copo}}$ für eine beliebige Mischung resultiert dann nicht nur aus einer Kontrolle durch die letzten („,terminal“) oder zusätzlich vorletzten (,penultimate“) Kettenglieder. Im $k_{\mathrm{t}, \text { copo }}$ spiegelt sich dann auch der Einfluss aller anderen Monomereinheiten auf die Entstehung von 
„mid-chain“ Radikalen und damit die Kettenlängenabhängigkeit von $k_{\mathrm{t}}$ wieder. Allerdings geben die Populationen $P_{\mathrm{i}}$ bzw. $P_{\mathrm{ij}}$ der Radikale nach dem „terminal model“ bzw. „penultimate model“ (vgl. Kapitel 7) auch die Anteile der Comonomere in der gesamten wachsenden Kette an, so dass eine Modellierung mit diesen Populationen korrekte Voraussagen liefern kann

\subsection{Literatur Kapitel 8}

[1] H. Fischer, R. Baer, R. Hany, I. Verhoolen, M. Walbiner, J. Chem. Soc., Perkin Trans. $1990,2,787$.

[2] P. Vana, T. P. Davis, C. Barner-Kowollik, J. Polym. Sci., Part A: Polym. Chem. 2002, $40,675$.

[3] M. Buback, M. Busch, C. Kowollik, Macromol. Theory Simul. 2000, 9, 442.

[4] A. Külpmann, Diplomarbeit, Göttingen 2000.

[5] P. Vana, T. P. Davis, C. Barner-Kowollik, Aust. J. Chem. 2002, 55, 315.

[6] M. Busch, A. Wahl, Macromol. Theory Simul. 1998, 7, 217.

[7] Polymer Handbook, $3^{\text {rd }}$ ed.; A. Bandrup, E. H. Immergut, Eds, Wiley-Interscience, New York 1989.

[8] J. B. L. de Kock, B. Klumpermann, A. M. van Herk, A. L. German, Macromolecules 1997, 30, 6743 .

[9] J. B. L. de Kock, Dissertation, Eindhoven 1999.

[10] K. Horie, I. Mita, H. Kambe, Polym. J. 1973, 4, 341.

[11] H. K. Mahabadi, K. F. O’Driscoll, J. Polym. Sci., Polym. Chem. Ed. 1977, 15, 283.

[12] T. Yasukawa, T. Takahashi, K. Murakami, Macromol. Chem. 1973, 174, 235.

[13] O. F. Olaj, G. Zifferer, G. Gleixner, Macromol. Chem., Rapid Commun. 1985, 6, 773.

[14] O. F. Olaj, G. Zifferer, G. Gleixner, Macromol. Chem. 1986, 187, 977.

[15] O. F. Olaj, G. Zifferer, G. Gleixner, Macromolecules 1987, 20, 839.

[16] A. R. Khokhlov, Macromol. Chem., Rapid Commun. 1981, 2, 633.

[17] P. G. de Gennes, J. Chem. Phys. 1982, 76, 3316 und 3322.

[18] M. Buback, C. Barner-Kowollik, M. Egorov, V. Kaminsky, Macromol. Theory Simul. 2001, 10, 209.

[19] P. Vana, T. P. Davis, C. Barner-Kowollik, Macromol. Rapid Commun. 2002, 23, 952. 
[20] A. J. Beckwith, D. Giller, J. P. Lorand, in: "Landolt-Börnstein, Numerical Data and Functional Relationships in Science and Technology, New Series", Vol. 13, H. Fischer, Ed., Springer-Verlag Berlin, Heidelberg, New York, Tokyo 1984, Part a, Chapter 1.

[21] H. K. Mahabadi, Macromolecules 1985, 18, 1319.

[22] O. F. Olaj, P. Vana, Macromol. Rapid Commun. 1998, 19, 433 und 533.

[23] B. Friedman, B. O’Shaughnessy, Macromolecules 1993, 26, 5726.

[24] E. Karatekin, B. O’Shaughnessy, N. J. Turro, Macromol. Symp. 2002, 182, 81.

[25] M. J. Roedel, J. Am. Chem. Soc. 1953, 75, 6110.

[26] A. G. Morrell, Discussions Faraday Soc. 1956, 22, 152.

[27] G. E. Scott, E. Senogles, J. Macromol. Sci.-Chem. 1974, A8 (4), 753.

[28] N. M Ahmad, F. Heatley, P. A. Lovell, Macromolecules 1998, 31, 2822.

[29] D. Britton, F. Heatley, P. A. Lovell, Macromolecules 2001, 34, 817.

[30] C. Plessis, G. Arzamendi, J. R. Leiza, H. A. S. Scoonbrood, D. Charmot, J. M. Asua, Macromolecules 2000, 33, 5041.

[31] C. Plessis, G. Arzamendi, J. R. Leiza, J. M. Alberti, H. A. S. Scoonbrood, D. Charmot, J. M. Asua, Macromolecules 2001, 34, 5147.

[32] C. Plessis, G. Arzamendi, J. R. Leiza, H. A. S. Scoonbrood, D. Charmot, J. M. Asua, Macromolecules 2001, 34, 4.

[33] B.Yamada, M. Azukizawa, H. Yamazoe, D. J. T. Hill, P. J. Pomery, Polymer 2000, 41, 5611.

[34] F. Heatley, P. A. Lovell, T. Yamashita, Macromolecules 2001, 34, 7636.

[35] M. Azukizawa, B.Yamada, D. J. T. Hill, P. J. Pomery, Macromol. Chem. Phys. 2000, $201,774$.

[36] C. Plessis, G. Arzamendi, J. M. Alberti, A. M. van Herk, J. R. Leiza, J. M. Azua, Macromol. Rapid Commun. 2003, 24, 173.

[37] C. Plessis, G. Arzamendi, J. R. Leiza, H. A. S. Scoonbrood, D. Charmot, J. M. Asua, Ind. Eng. Chem. Res. 2001, 40, 3883.

[38] S. Beuermann, M. Buback, F.-D. Kuchta, C. Schmaltz, Macromol. Chem. Phys. 1998, 199, 1209.

[39] S. Beuermann, M. Buback, C. Isemer, A. Wahl, Macromol. Rapid Commun. 1999, 20, 26.

[40] S. Beuermann, M. Buback, C. Schmaltz, Macromol. Chem. Phys. 1998, 199, 8069.

[41] S. Beuermann, M. Buback, C. Schmaltz, Ind. Eng. Chem. Res. 1999, 38, 3338. 
[42] S. Beuermann, M. Buback, D. Nelke, Macromolecules 2001, 34, 6637.

[43] S. Beuermann, M. Buback, C. Isemer, I. Lacík, A. Wahl, Macromolecules 2002, 35, 3866.

[44] D. Nelke, Dissertation, Göttingen 2002.

[45] C. Schmaltz, Dissertation, Göttingen 1997.

[46] C. Kurz, Dissertation, Göttingen 1995.

[47] C. Kowollik, Dissertation, Göttingen 1999.

[48] R. A. Hutchinson, J. H. McMinn, D. A. Paquet Jr., S. Beuermann, C. Jackson, Ind. Eng. Chem. Res. 1997, 36, 1103.

[49] M. Buback, A. Feldermann, C. Barner-Kowollik, I. Lacík, Macromolecules 2001, 34, 5439.

[50] M. Buback, A. Feldermann, Aust. J. Chem. 2002, 55, 475.

[51] T. Junkers, unveröffentlichte Ergebnisse, Göttingen 2003. 


\section{Ausblick}

Die Messungen zur Terminierungskinetik der MMA-Homopolymerisation haben gezeigt, dass durch eine thermische Vorpolymerisation vor dem eigentlichen SP-PLP-Experiment das Erreichen höherer Monomerumsätze in homogener Phase möglich wird. Es erscheint deshalb interessant, dieses Verfahren auch für andere Monomer mit eingeschränktem zugänglichen Umsatzbereich, wie etwa MA, anzuwenden. Bei den MMA-Homopolymerisationen wurde ebenfalls der Einsatz des Molmassenreglers DDM untersucht. Diese Experimente sollten dahingehend erweitert werden, dass die Reglerkonzentration variiert und dadurch die Polymermolmasse eingestellt wird. Zudem könnten verschiedene Übertragungsagenzien eingesetzt werden, wobei auch katalytisch wirkende Molmassenregler in Betracht zu ziehen sind. Allerdings ergab sich bei einigen Messungen mit Vorpolymerisation das Problem von Inhomogenität der Reaktionsmischung. Ein anderer Ansatz für Messungen bei höheren Umsätzen ist deshalb das Mischen von Polymer mit Monomer, um auf diese Weise entsprechende Umsätze zu simulieren. Dabei kann zusätzlich die Molmasse des Polymers und damit auch die Viskosität der Mischung gezielt variiert und der Einfluss auf $k_{\mathrm{t}}$ studiert werden.

Bei den Untersuchungen zur Copolymerisation von Methacrylat-Acrylat Systemen hat sich gezeigt, dass die Änderung der aktuellen Monomerzusammensetzung bei höheren Monomerumsätzen durchaus signifikant wird. Dieser Effekt wirkt sich besonders bei Mischungen mit niedrigem Acrylat-Anteil auf $k_{\mathrm{p}}$ und damit auch auf $k_{\mathrm{t}}$ aus und sollte bei der Modellierung der Terminierungsgeschwindigkeitskoeffizienten beachtet werden. Somit wäre auch bei anderen bereits untersuchten Systemen wie MMA-BA [1] und DMA-DA [2] eine entsprechende Analyse der vorhandenen Daten wünschenswert. Die Anwendbarkeit des bei den in dieser Arbeit betrachteten Copolymerisationssystemen erfolgreichen „penultimate“ Modells sollte durch Untersuchung weiterer Systeme überprüft werden. Dazu bietet sich die Kombination eines langsam wachsenden Monomers wie etwa Styrol mit DA oder MA an. Bei allen Analysen von Copolymerisationen muss zusätzlich die Kettenlängenabhängigkeit von $k_{\mathrm{t}}$ durch die Auswahl geeigneter Signalaufnahmezeiten berücksichtigt werden.

Mit der SP-PLP-Technik lässt sich bei Verwendung eines geeigneten Photoinitiators wie etwa MMMP die Kettenlängenabhängigkeit von $k_{\mathrm{t}}$ aus einzelnen Messsignalen modellabhängig bestimmen. Aufgrund der unzureichenden Signalqualität ist mit SP-PLP eine modellunabhängige Ermittlung oder eine Modelldiskriminierung nicht möglich. Die Ergebnisse 
deuten darauf hin, dass für das Ausmaß der Kettenlängenabhängigkeit die Bildung von Radikalen mit aktivem Zentrum in der Mitte der Kette (,mid-chain“ Radikale) verantwortlich ist. Dieser Befund sollte aber durch den Nachweis von verzweigtem Polymer mit unabhängigen Techniken, wie etwa NMR, bestätigt werden. Zur Erhöhung der Genauigkeit der Anpassungen des verwendeten Potenzgesetzes an die Messsignale wäre es wünschenswert, einen der Parameter unabhängig zu bestimmen. So kann $k_{\mathrm{t}}^{0}$ durch gezielte Variation der Radikalkettenlänge mit einem Übertragungsagens und Extrapolation auf sehr kleine Kettenlängen abgeschätzt werden. Eine Alternative bietet die von Vana et al. [3] vorgeschlagene Auswertung von Daten aus „kontrollierter/lebender radikalischer Polymerisation“.

Durch die Kombination von PLP mit Elektronenspinresonanz (ESR) könnte direkt die Radikalkonzentration nach einem einzelnen Laserpuls zeitaufgelöst verfolgt werden. Dadurch wäre eine modellunabhängige Bestimmung der Kettenlängenabhängigkeit von $k_{\mathrm{t}}$ möglich. Erste Experimente mit dieser Technik sind vielversprechend [4].

\section{Literatur Kapitel 9}

[1] C. Kowollik, Dissertation, Göttingen 1999.

[2] M. Buback, C. Kowollik, Macromolecules 1999, 32, 1445.

[3] P. Vana, T. P. Davis, C. Barner-Kowollik, Macromol. Rapid Commun. 2002, 23, 952.

[4] M. Buback, T. Junkers, E. Panchenko, unveröffentlichte Ergebnisse 2002. 


\section{ANHANG}

\subsection{Verzeichnis der Abkürzungen}

A

AIBN

$\alpha$

$B$

BA

BMA

$c_{\mathrm{I}}$

$c_{\mathrm{M}}$

$c_{\mathrm{M}}{ }^{0}$

$c_{\mathrm{R}}$

$c_{\mathrm{R}}{ }^{0}$

$C_{\mathrm{RD}}$

$d$

DA

DDM

DMA

DMPA

$D^{*}$

$\Delta V^{\ddagger}$

$E_{\mathrm{A}}$

$E_{\lambda}$

$E_{\mathrm{P}}$

$\varepsilon$

$\eta$

$\eta_{\mathrm{r}}$

$\eta$

$f$

$f_{\mathrm{i}}$

$f_{\mathrm{i}}^{0}$
Absorbanz

Azo-bis-isobutyronitril

Koeffizient der Kettenlängenabhängigkeit der Terminierungsreaktion integrale Molabsorptivität

Butylacrylat

Butylmethacrylat

Initiatorkonzentration

(Gesamt) Monomerkonzentration

Startmonomerkonzentration

stationäre Radikalkonzentration

Startradikalkonzentration

Parameter der Reaktionsdiffusion

Schichtlänge

Dodecylacrylat

$n$-Dodecylmercaptan

Dodecylmethacrylat

2,2-Dimethoxy-2-phenylacetophenon

Detektivität

Aktivierungsvolumen

Aktivierungsenergie

Energie eines Mols Photonen bei der Laserwellenlänge $\lambda$

Energie eines Laserpulses

molarer dekadischer Absorptionskoeffizient

Viskosität der Lösung

relative Viskosität

Viskosität der Lösung bei $0 \%$ Monomerumsatz

Initiatoreffektivität

Molenbruch der Komponente i

Molenbruch der Komponente i zu Beginn der Polymerisation 
$F_{\mathrm{i}} \quad$ Molenbruch des Monomeren i im binären Copolymer

$\varphi \quad$ Lasereffektivität

$\Phi \quad$ Primärquantenausbeute

GPC Gelpermeatationschromatographie

$I_{0} \quad$ Intensität des auf die Probe auftreffenden Lichts

I Intensität des aus der Probe austretenden Lichts

$i, j \quad$ Polymerkettenlängen

$k_{\mathrm{CR}} \quad$ Anteil der Reaktionsdiffusion zum Terminierungsgeschwindigkeits-

koeffizienten der Homopolymerisation

$k_{\mathrm{d}} \quad$ Dissoziationskonstante des Initiators

$k_{\mathrm{p}} \quad$ Wachstumsgeschwindigkeitskoeffizient der Homopolymerisation

$k_{\mathrm{p}, \text { соро }} \quad$ (mittlerer) Wachstumsgeschwindigkeitskoeffizient der binären

Copolymerisation

$k_{\mathrm{p}, \mathrm{D}} \quad$ diffusionskontrollierter Beitrag von $k_{\mathrm{p}}$

$k_{\text {pii }} \quad$ Wachstumsgeschwindigkeitskoeffizient der Homopolymerisation des

Monomeren i

$k_{\mathrm{pij}} \quad$ Wachstumsgeschwindigkeitskoeffizient der Reaktion des Makroradikals mit der Endeinheit i mit dem Monomeren vom Typ j

$k_{\mathrm{pijk}} \quad$ Wachstumsgeschwindigkeitskoeffizient der Reaktion des Makroradikals mit der vorletzten Einheit i und der letzten Einheit j mit dem Monomeren k

$k_{\mathrm{p}, 0} \quad k_{\mathrm{p}}$ ohne diffusionskontrollierten Anteil

$k_{\mathrm{t}} \quad$ Terminierungsgeschwindigkeitskoeffizient

$\left\langle k_{\mathrm{t}}\right\rangle \quad$ (kettenlängengemittelter) Terminierungsgeschwindigkeitskoeffizient

$k_{\mathrm{t}}^{0} \quad$ Terminierungsgeschwindigkeitskoeffizient für sehr kleine Radikale

$k_{\mathrm{t}, \mathrm{copo}} \quad$ (Kettenlängen gemittelter) Terminierungsgeschwindigkeitskoeffizient der binären Copolymerisation

$k_{\mathrm{t}, \mathrm{D}} \quad$ (diffusionsanhängiger) Terminierungsgeschwindigkeitskoeffizient der Homopolymerisation ohne Anteil von Reaktionsdiffuison

$k_{\mathrm{TD}} \quad$ Anteil der Translationsdiffusion zum Terminierungsgeschwindigkeitskoeffizienten der Homopolymerisation

$k_{\mathrm{t}}(\mathrm{i}, \mathrm{i}) \quad$ Koeffizient der Terminierungsreaktion zweier Makroradikale der Kettenlänge $\mathrm{i}$

$k_{\mathrm{t}}(\mathrm{i}, \mathrm{j}) \quad$ Koeffizient der Terminierungsreaktion zweier Makroradikale der Kettenlänge i und $\mathrm{j}$ 
$k_{\mathrm{t} i \mathrm{j}, \mathrm{kl}} \quad$ (Kettenlängen gemittelter) Terminierungsgeschwindigkeitskoeffizient der

Reaktion des Makroradikals mit der vorletzten Einheit i und der letzten Einheit j mit dem Makroradikal mit der vorletzten Einheit k und der letzten Einheit 1

$k_{\text {tr }} \quad$ Koeffizient der Kettenübertragungsreaktion

$k_{\mathrm{SD}} \quad$ Anteil der Segmentdiffusion zum Terminierungsgeschwindigkeitskoeffizientenen der Homopolymerisation

M Molmasse

$\cdot \mathrm{M}_{2}$. Dimeres Biradikal

MA Methylacrylat

MMA Methylmethacrylat

MMMP $\quad \alpha$-Methyl-4(methylmercapto) $-\alpha-$ morpholinopropiophenon

$n_{\text {abs }} \quad$ Anzahl der absorbierten Photonen

NIR nahes Infrarot

$v \quad$ Laserfrequenz

p Druck

$P_{\mathrm{i}} \quad$ relative Population eines Makroradikals mit der letzten Einheit i

$P_{\mathrm{ij}} \quad$ relative Population eines Makroradikals mit der vorletzten Einheit i und der letzten Einheit j

PLP-SEC Pulsed Laser Polymerization-Size Exclusion Chromatography

PS-PLP Pulse Sequence-Pulsed Laser Polymerization

R. Makroradikal

$\mathrm{R}_{\mathrm{i}} \quad$ Makroradikal mit der Endeinheit i

$\mathrm{R}_{\mathrm{ij}} \quad$ Makroradikal mit der vorletzten Einheit i und der letzten Einheit $\mathrm{j}$

$r_{\mathrm{i}} \quad$ Copolymerisationparameter (Homo- zu Kreuzwachstum)

$\mathrm{scCO}_{2} \quad$ überkritisches $\mathrm{CO}_{2}$

$s_{\mathrm{i}} \quad$ Radikalcopolymerisationsparameter

SP-PLP Single Pulse-Pulsed Laser Polymerization

$t \quad$ Zeit

$\tau_{1 / 2} \quad$ Halbwertszeit

$T \quad$ absolute Temperatur

X Monomerumsatz

$v_{\mathrm{p}} \quad$ Wachstumsgeschwindigkeit 


\subsection{Betriebsanweisung der Anlage zur Aufnahme zeitaufgelöster IR- Signale (SP-PLP)}

Im Rahmen der vorliegenden Arbeit wurde die SP-PLP-Anlage durch den Einbau einer neuen Transientenrekorderkarte modifiziert. Dabei wurden die Programme zur Datenaufnahme und Auswertung neu erstellt. Diese Betriebsanweisung dokumentiert die durch die Modifikation veränderte Bedienung der Anlage.

\section{Inhaltsverzeichnis}

1. Eichung des Lasers

2. Vorbereitung der Probesubstanzen/Messung

2.1 Entgasen der Probesubstanz

2.2 Einstellungen der Mess-/Aufnahmeprogramme

2.3 Aufbau der Innenzelle

3. Ablauf der Messung

3.1 Selektierung des Peakmaximums über die Monochromatorsteuerung (Atari)

3.2 Aufnahme der Signaldaten

3.3 Aufnahme der Vergleichsspektren

$3.4 \quad$ Zusammenfassung

4. Auswertung der Messdaten

4.1 Berechnung des Extinktionskoeffizienten

4.2 I008-Korrektur

4.3 Exportieren der Signaldaten

4.4 Anpassung der Signaldaten

5. Aktueller Programmcode 


\section{Eichung des Lasers}

Vor Beginn der Messung muss der Spitzenwertdetektor (UV-Detektor) gegen die Energie des Eximerlaserstrahls (Lextra 50 Eximer-Laser, XeF) hinter der Hochdruckzelle geeicht werden. Dazu wird die Hochdruckzelle mit einer leeren Innenzelle bestückt (nur zwei Quarzfenster mit entsprechend langem Teflonschlauch) und mit dem Druckmittel auf Messdruck eingestellt. Die Hochdruckzelle wird in den Strahlengang gesetzt. Der EnergieDetektor (GenTec) wird hinter der Zelle plaziert und angeschlossen. Es ist zu beachten, dass die die Optik tragende Marmorplatte nicht auf den Holzklötzen aufliegen darf und vollständig eben gelagert ist. Gegebenenfalls muss an den Schubkarrenschläuchen nachgeregelt werden. Der Laser sollte 10 min im Vorfeld ,warmgelaufen“ sein, d.h. mit einer Frequenz von $1 \mathrm{~Hz}$ bei mittlerer Laserenergie pulsen. (Einstellung Trigger Intern in Lasersteuerung). Zur eigentlichen Eichung wird das C-Programm

\section{"Eichung.cpp"}

am PC gestartet und Nutzername sowie Datum eingegeben. Es wird dann im Nutzerverzeichnis \Eichung ein logfile mit dem Datum als Dateiname angelegt. In der Lasersteuerung wird die höchste Energieleistung (24 kV, Trigger Extern) selektiert und der Laser gestartet. Das Eichprogramm startet die Eichung mit $1 \mathrm{~Hz}$ Pulsfrequenz, nachdem die Anzahl der Eichpulse (100) eingegeben worden ist. Das Eichprogramm kann die Laserleistung nicht steuern, daher muss nach je 25 Pulsen per Handeingabe am Laser PC die Leistung um 1 $\mathrm{kV}$ gesenkt werden. Das logfile, dass die Eichdaten gespeichert hat, wird mit dem Programm

\section{XACT}

eingelesen und es wird eine lineare Regression durchgeführt. Anhand der Regressionsdaten wird die gewünschte Laserenergie abgelesen (im Regelfall werden $2.5 \mathrm{~mJ}$ für SP-PLP Messungen verwendet) Ist die Laserenergie zu niedrig oder schlecht korreliert, muss der Laser neu befüllt (siehe Bedienungshandbuch des Lasers) und die Eichung wiederholt werden. 


\section{Vorbereitung der Probesubstanz/Messung}

\subsection{Entgasen der Probesubstanz}

Die Probesubstanz muss für die Messung von Sauerstoff befreit werden. Dazu wird das Monomer sowie alle weiteren flüssigen Substanzen (Initiator/Tranferagens) in einem dafür vorgesehenen Entgasungskolben eingewogen (kann bereits am Vortag geschehen). Die Probelösung wird dann an die Turbomolekularpumpe angeschlossen und bei geschlossenem Ventil mit Stickstoff 15 min eingefroren. Anschließend wird das Ventil unter weiterer Kühlung geöffnet. Auf die Dichtigkeit des Kolbens ist zu achten ( $\mathrm{p} \ll<1$ torr). Ist ein konstanter Druck erreicht, wird das Ventil geschlossen und die Probesubstanz in einem Wasserbad bei RT aufgetaut. Dieser Zyklus wird insgesamt dreimal durchgeführt.

\subsection{Einstellungen der Mess/Aufnahmeprogramme}

Zur Aufnahme von Messsignalen werden verschiedene Programme am PC und Atari verwendet (Atari siehe nächster Abschnitt) Am PC muss das C-Programm

\section{"Messung"}

von Desktop und

\section{Sbench v.4}

geöffnet sein. Das eigentliche Messprogramm (C-Programm) dient zur Aufnahme von Spannungswerten vom IR-Detektor vor und nach den einzelnen Laserpulspaketen, sowie von Messparametern wie z.B. Anzahl an Pulsen und Laserenergie am UV-Detektor (korreliert mit Laserenergie an der Zelle, siehe Lasereichung). Die Bedienung des Programms ergibt sich aus den Abfragen.

Das Programm Sbench nimmt die eigentlichen Messsignale auf. Dazu müssen im Programm einige Einstellungen vorgenommen werden, von denen die meisten bereits in einer Vorlage (Setup laden, setup.cfg auf apc24 \benutzer) gespeichert sind. Es können verschiedene Fenster geöffnet werden: 
a) AutoSave (Speicherung der Messdaten)

Mittel\# 1 selektieren, dann klick auf setup

- Dateiname: Name der Messfiles

- Startindex: Die Messsignale werden automatisch durchnummeriert, der Startindex gibt an, welche Laufnummer die erste Datei erhält.

- Intervall $=1$

- Das Kontrollkästchen Signale an/aus muss aktiviert sein.

- Dateiformat "Sbench4.x*.dat

Durch Klicken auf OK wird das Fenster geschlossen, zur Aktivierung der Eingaben muss Mittel\# 1 mit " Start" bestätigt werden. Es erscheint die Meldung „warte update“.

b) Mittelung \# 1

Neben dem Messprogramm muss auch bei Sbench eingegeben werden, über wie viele Laserpulse gemittelt werden soll. Dafür sind folgende Einstellungen erforderlich:

- Quelldatei: PAD

- Ziel Mittel\# 1

- Durchläufe/Update auf gleiche Anzahl einstellen (=Anzahl der Laserpulse).

- Automatischer Neustart muss aktiviert sein.

- Durch Klicken auf Start werden die Einstellungen aktiviert (wichtig: nur wenn alle Pulse eingegangen sind, wird das Signal gespeichert. Alle Laserpulse, die vor oder nach dem angegebenen Intervall gezählt werden, werden entweder nicht gespeichert oder tragen zur Mittelung eines anderen Signalpaketes bei. Durch " abbrechen" werden die aktuellen Daten vollständig gelöscht und nicht gespeichert).

c) PAD

Dieses Fenster steuert die Grundfunktionen der Transientenrekorderkarte.

- In der ersten Zeile wird Input 1 ausgewählt.

- Der Eingangsbereich ist standardmäßig + /-1 V. Änderungen müssen auch im Messprogramm Messung.cpp übernommen werden.

- Die Abtastrate gibt die zeitliche Differenz zwischen zwei Messpunkten an.

- Die Speichertiefe ist standardmäßig auf 65537 eingestellt, ein Punkt fehlt in der Auswertung, deshalb $2^{16}+1$. 
- Davon entfallen $75 \%$, also 49153 auf den Posttrigger.

- Der Triggermodus ist TTL negativ.

Dieses Fenster wird nicht über " start" aktiviert. Die Aktivierung erfolgt über Drücken des Buttons " Repeat" im Sbench-Hauptfenster. Dadurch wird das Programm zur Datenaufnahme " scharf" gemacht

Vor Drücken des " repeat" -Buttons sollte das Ansichtsfenster für die Messsignale geöffnet werden, da es sonst zu Komplikationen mit dem Aufnahmeprogramm kommen kann. Dazu wird in " Anzeige" " Zeit-Fenster" Nr. 1 ausgewählt. Dadurch öffnet sich ein weiteres Fenster, in dem unter " Einstellungen" , " Signale +/-" Mittel\# 1 markiert und mit OK bestätigt wird. Im setup.cfg file ist dieses Fenster schon vorhanden.

\subsection{Aufbau der Innenzelle}

Die Innenzelle wird in der Trockenbox zusammengesetzt. Wichtig ist die Schichtlänge der Innenzelle, die vor Einbau in die Hochdruckzelle mit einer Mikrometerschraube zu messen ist. Die Zellenlänge sollte an die verwendete Hochdruckzelle angepasst werden. Für die Cko Zelle sollte die Zelle nicht länger als $11.50 \mathrm{~mm}$ sein. Eine möglichst große Schichtlänge ist sinnvoll, jedoch muss berücksichtigt werden, dass sich die Zelle je nach Messbedingungen ausdehnen kann.

\section{Ablauf der Messung}

\subsection{Selektierung des Peakmaximums über Monochromatorsteuerung (Atari)}

Befindet sich die auf Messbedingungen eingestellte Hochdruckzelle mit gefüllter Innenzelle im Strahlengang der SP-PLP Anlage, muss zu Beginn das Peakmaximum der zu beobachtenden IR-Bande selektiert werden. Dafür müssen die IR-Lampe und der Detektor angeschaltet und die IR-Blenden offen sein (Öffnen der IR-Blenden durch Starten des Messprogramms). Am Atari wird im Verzeichnis „Messen“ die Datei

\section{"MESSENMO"}


zur Monochromatorsteuerung gestartet. Die Einstellung einer bestimmten Wellenzahl ist nicht direkt möglich, es wird nur das Absorptionsmaximum in einem bestimmten Bereich gesucht. In der Monochromatorsteuerung wird "Intensität" ausgewählt, und der Drehregler am Detektor so geregelt, dass die Intensitätsanzeige ca. in der Mitte des Bildschirms des Atari zu finden ist. Dann wird durch Auswahl der Option ,zu kleineren Werten“ der Bereich um einen bestimmten Wert verschoben (in der Regel 15 Schrittstufen, muss jedoch nach Bedarf angepasst werden). Anschließend sucht der Atari selbständig durch Auswählen von „Peakmaximum suchen zu höheren Wellenzahlen“ (Eingabe muss einen größeren Wert haben als vorher zurückgedreht wurde) das Maximum der IR-Bande und stellt diesen Wert ein. Der Zähler am Monochromator zeigt für Peakmaxima um $6170 \mathrm{~cm}^{-1}$ einen Wert von etwa 807 an. Nach Selektion des Peakmaximums wird die Intensität am Atari wieder auf ca. 0.15$0.20 \mathrm{~V}$ eingestellt.

Die Intensitätsangabe am Atari ist nicht identisch mit den aufgenommenen Daten, die später in der Auswertung berücksichtigt werden, da es sich um unterschiedliche Ausgangskanäle des Detektors handelt. Der Intensitätswert am Atari (nahezu identisch mit der Ausgabe des Messprogramms als Kontrollwert zwischen den Laserpulsen) dient nur zur Kontrolle während der Messung und wird nicht gespeichert.

ACHTUNG: Die Spaltbreiten am Monochromator müssen auf beiden Seiten identisch sein, da die Messsignale sonst nicht ausgewertet werden können. Sinnvollerweise sind die Spaltbreiten vor der Selektierung des Peakmaximums festzulegen (Abschätzung der Größe des Spaltes siehe 3.2)

\subsection{Aufnahme der Signaldaten}

Ist die Hochdruckzelle unter Messbedingungen und Sbench sowie das C-Programm „Messung“ gestartet, kann mit der Aufnahme von Messsignalen begonnen werden. Es ist den Abfragen des C-Programms zu folgen, während Sbench wie oben beschrieben eingestellt wird. Zu Beginn der Abfragen wird der sogenannte I008-Wert aufgenommen und notiert. Es ist $\mathrm{zu}$ beachten, dass nach Einschalten der IR-Lampe und des Detektors der Wert noch schwanken kann, da die Geräte erst warmlaufen müssen. Daher sollte der I008-Wert mehrfach gemessen werden, und erst bestätigt werden, wenn er auf mindestens 3 Nachkommastellen konstant ist. Der I008-Wert sollte zwischen 0.15 und 0.25 liegen. Bei zu niedrigem Wert ist die Lichtintensität für ein gutes Signal zu gering, bei einem zu hohen Wert erfolgt die 
Kompensation des Messsignals nur unzureichend. Die Größe dieses Wertes kann durch Veränderung der Spaltbreiten am Monochromator variiert werden.

Nach Überprüfung dieses Startwertes wird der Laser " scharf" geschaltet und die Aufnahme kann begonnen werden. Die Dateien werden fortlaufend durchnummeriert und unter angegebenen Dateinamen im Nutzerverzeichnis abgespeichert. Die weitere Bedienung des Messprogramms ergibt sich aus den Abfragen. Per Hand muss notiert werden, mit welcher Abtastrate die Daten aufgenommen werden (kHz-Zahl in Sbench) und bei welcher Spaltbreite gemessen wurde.

Während der Messungen kann die Intensität am Atari verfolgt werden. Es ist darauf zu achten, dass der Spannungswert zwischen den Laserpulsen konstant ist. Ist dies nicht der Fall, sind die Signale nicht oder nur sehr eingeschränkt auswertbar. Anmerkung: Bis auf die Abtastrate und die Spaltbreite werden alle erforderlichen Daten im outputfile des Messprogramms gespeichert. Es ist jedoch sinnvoll, zusätzliche Parameter per Hand zu protokollieren, wie etwa Anzahl der Laserpulse.

\subsection{Aufnahme der Vergleichsspektren}

Durch die stark unterschiedlichen Messmethoden (kleiner Spalt bei der SP-PLP-Anlage und vollständiges Spektrum bei FT-IR) unterscheiden sich die bei der Auswertung vom Messprogramm gespeicherten Daten bezüglich des Umsatzes von den FT-IR-Spektren. Daher sollten im Abstand von $5 \%$ Monomerumsatz Vergleichspektren am FT-IR-Gerät aufgenommen werden. Zusätzlich zu diesen Vergleichsspektren ist unbedingt vor der Messung ein Leerspektrum (Hochdruckzelle mit leerer Innenzelle, ohne Druckmittel) aufzunehmen. Dies geschieht sinnvollerweise noch vor der Lasereichung. Bevor das erste Messsignal aufgenommen wird, muss ebenfalls ein Startspektrum bei Messbedingungen aufgenommen werden. Dies ist notwendig, um den Extinktionskoeffizienten zu bestimmen, ohne den aus den Daten der zeitaufgelösten Messung kein Umsatz bestimmt werden kann. 


\subsection{Zusammenfassung}

Zeitlicher Ablauf der Messung:

1) Aufnahme eines Leerkanalspektrums

2) Lasereichung

3) Zeitgleich zu 1) und 2): Entgasungszyklus der Probesubstanzen

4) Aufnahme des Startspektrums

5) Einstellen der Spaltbreite

6) Selektion des Peakmaximums

7) Einstellen der Messparameter

8) Messung/Aufnahme von Vergleichsspektren

Während der Messung muss protokolliert werden:

1) Schichtlänge der Innenzelle

2) Integral des Startspektrums

3) Peakmaximum des Startspektrums

4) Spaltbreite am Monochromator

5) Dateinamen

6) Signallänge (s. Sbench), d.h. Abtastrate und Speichertiefe

7) Integrale der Vergleichsspektren

optional/empfohlen:

1) Anzahl der Pulse pro Paket

2) I008-Wert

3) Eingestellte Hochspannung am Laser zum Erreichen gewünschter Laserenergie 


\section{Auswertung der Messdaten}

\subsection{Berechnung des Extinktionskoeffizienten}

Die Berechnung des Extinktionskoeffizienten $\varepsilon_{\text {eff }}$ erfolgt über Daten aus dem Programm „OPUS“. Es wird für das vor Aufnahme des ersten Messsignals gemessene Startspektrum eine Bandensuche zur Bestimmung der Lage des Peakmaximums bei etwa $6170 \mathrm{~cm}^{-1}$ durchgeführt. Aus der Lage der Bande wird der durch den Monomchromatorspalt vorgegebene Spektralbereich ermittelt. Die Spaltbreite in $\mathrm{mm}$ wird halbiert und mit der Gitterkonstante von $4.1 \cdot 10^{-9}$ (in $\mathrm{nm} / \mathrm{mm}$ ) multipliziert. Dieser Wert wird hier mit $\mathrm{X}$ bezeichnet. Der Spektralbereich ergibt sich aus folgender Rechnung:

$\left(\left(\text { Peakmaximum }\left(\text { in } \mathrm{cm}^{-1}\right)\right)^{-1} / 100-\mathrm{X}\right)^{-1} / 100$

obere Grenze

$\left(\left(\text { Peakmaximum }\left(\text { in }^{-1}\right)\right)^{-1} / 100+\mathrm{X}\right)^{-1} / 100$

untere Grenze

Das Startspektrum wird in den erhaltenen Grenzen integriert. Der Integrationswert geteilt durch den Bereich ergibt die effektive Absorbanz $A_{\text {eff. }} \varepsilon_{\text {eff }}$ ergibt sich aus Division von $A_{\text {eff }}$ durch Konzentration der Messlösung und Schichtlänge in cm.

\subsection{I008-Korrektur}

Optional können die vom Messprogramm gespeicherten Daten derart umgerechnet werden, dass die sich aus diesen Daten ergebenen Umsätze besser mit den aus den FT-IR Spektren bestimmbaren Umsätzen korreliert sind. Dazu werden zunächst die Spektren integriert und der jeweilige Monomerumsatz bestimmt. Die Umrechnung passt den im outputfile in der ersten Zeile gespeicherten Wert (I008, d.h. Spannung am Kanal 8 vor der Messung, siehe Programmcode) an die Werte in der zweiten (I08, d.h. Spannung am Kanal 8 vor dem Pulspaket) und fünften Zeile (I2, d.h. Spannung am Kanal 8 nach dem Pulspaket) sowie an die aus den Spektren bestimmten Umsätze an. Die Umsatzberechnung wird rückwärts durchgeführt.

In der Excel Datei „I008_ korrektur“ werden Schichtlänge, $\varepsilon_{\text {eff }}$, Molmasse, Dichte und die Umsätze aus der Analyse zweier aufeinander folgender Spektren eingetragen. Für die Signale, die zwischen diesen Spektren aufgenommen wurden, wird die I008-Korrektur durchgeführt. Aus dem outputfile des ersten Signals wird I08 und aus dem outputfile des 
letzten Signals I2 in die Excel Tabelle eingetragen. Der erhaltene I008 Wert ersetzt die erste Zeile aller zwischen den beiden Spektren erzeugten outputfiles.

\subsection{Exportieren der Signaldaten}

Die von „Sbench“ produzierten DAT files werden über „Datensatz laden“ eingelesen (Mittel\# 1 überschreiben) und sollten als Textfiles exportiert werden. Unter Datei-ExportASCII-1 wird ein entsprechendes Fenster geöffnet. Mittel\# 1wird markiert und die Punktanzahl über „Cursor“ ausgewählt (Cursor Position 0, ganzes Signal). Mit den Einstellungen „Datenformat 12.34 (Punkt)“ und „Dateikopf kein Header“ wird unter Export im auszuwählenden Ordner eine Textdatei erzeugt. Die Dateiextension .txt muss per Hand eingetragen werden.

\subsection{Anpassen der Signaldaten}

Die Auswertung der exportierten Signale erfolgt mit dem Programm „Matlab“ (und „Origin“). Das Matlab Programm „Auswert.m“ verbindet die Daten aus Sbench mit denen aus dem Messprogramm und berechnet $\mathrm{c}_{\mathrm{M}} / \mathrm{c}_{\mathrm{M}}{ }^{0}$ als Funktion der Aufnahmezeit.

In der Datei „Auswert.m“ müssen vor dem Programmstart die für die jeweilige Messung gültigen Parameter wie Schichtlänge, Abtastrate, Molmasse und Dichte eingetragen werden. Außerdem kann der Pretriggerbereich, in den eine horizontale „Basislinie“ vor dem Laserpuls gelegt wird, ausgewählt (Standard X=30) sowie die Anzahl der Datenpunkte für die Mittelung (Standard $\mathrm{n}=5$ ) bestimmt werden. Das Programm bietet eine Anpassung der idealen Polymerisationskinetik (Gleichung 3.6) an die Messdaten an und wird durch Eintippen von „Auswert“ in der Kommandozeile von Matlab gestartet. Dabei muss sich die Datei „Auswert.m“ im bei Matlab oben rechts auszuwählenden Verzeichnis (,current directory“) befinden.

Bei „Auswert.m“ werden zehn Anpassungen für jeweils $10 \%, 20 \%, \ldots, 100 \%$ des Signals errechnet und die erhaltenen Parameter in der Datei „Dateiname1“ (auch vorher zu ändern) gesichert. Durch Variation des Unterprogramms ,fitsignal“ lässt sich auch einstellen, dass nur $100 \%$ oder beliebige andere Bereiche gefittet werden. Eine Neufassung von „Auswert.m“ mit einer entsprechenden Abfrage ist in Vorbereitung. Bei erfolglosem Abbruch des Fits sollten die Startparameter q und w variiert werden. 
Neben Erzeugung der Textdatei werden in der Ausgabezeile von Matlab Werte wie der mittlere Monomerumsatz während des Pulspaketes angegeben.

In der Datei „Dateiname2“ werden die Daten von $\mathrm{c}_{\mathrm{M}} / \mathrm{c}_{\mathrm{M}}{ }^{0}$ als Funktion der Aufnahmezeit separat abgespeichert. Diese Datei kann nun in z.B. in Origin eingelesen und so Anpassungen der Signale an unterschiedliche Modelle durchgeführt werden, z.B. mit „myfitfunc3“ für Kettenlängenabhängigkeit von $k_{\mathrm{t}}$.

Alternativ zu „Auswert.m“ steht das Programm „Graphmaker.m“ zur Verfügung. In diesem Fall wird keine Anpassung durchgeführt, sondern es erfolgt nur die Erzeugung der Datei mit der relativen Monomerkonzentration als Funktion der Zeit.

\section{C-Programmcode}

\section{Programm zur Eichung des Excimerlasers}

// Programm zur Eichung des Lextra 50 UV-Lasers mit UV-Detektor hinter dem Laser. // Die Laserenergie kann als Funktion der Detektorspannung aufgetragen und die // Eichparameter eines linearen Fits an das Auswerteprogramm übergeben werden. // Damit wird zu jedem Laserpuls die entsprechende Laserenergie berechnet.

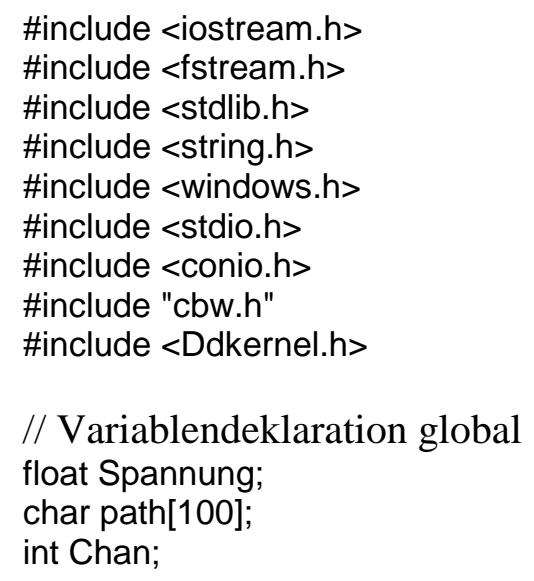

// Unterprogramm zur Bereitstellung eines Ordners für die Eichung mit Datumseingabe void Eingabe() \{

//Variablendeklaration lokal char name[100]; char datum[100]; char dir[100];

// Namen für Ordner einlesen cout $<<$ "Benutzername eingeben" $<<$ endl; cin $>>$ name; strcpy (dir, "||benutzer $\backslash ")$; 
strcat (dir, name);

strcat (dir, "\|Eichung \");

cout $<<$ "Datum eingeben" $<<$ endl;

cin $>>$ datum;

// Ordner gemäß der Eingabe erstellen

_makepath(path, "d", dir, datum ,"txt" );

\}

// Unterprogramm, um Spannung an Kanal 0 oder 1 einzulesen

void Einlesen() \{

int BoardNum = 0;

int UDStat = 0;

int Gain = UNI10VOLTS;

WORD DataValue = 0;

float RevLevel = (float)CURRENTREVNUM;

/* Declare UL Revision Level */

UDStat $=$ cbDeclareRevision (\&RevLevel);

/* Initiate error handling

Parameters:

PRINTALL :all warnings and errors encountered will be printed

STOPALL :if any error is encountered, the program will stop */

cbErrHandling (PRINTALL, STOPALL);

/*Parameters:

BoardNum :number used by CB.CFG to describe this board

Chan :input channel number

Gain :gain for the board in BoardNum

DataValue :value collected from Chan */

UDStat = cbAln (BoardNum, Chan, Gain, \&DataValue);

UDStat $=\mathrm{cbToEngUnits} \mathrm{(BoardNum,} \mathrm{Gain,} \mathrm{DataValue,} \mathrm{\& Spannung);}$

\}

void main() \{

Eingabe();

char *Datenkopf=("Puls U/V E/mJ");

int $\mathrm{n}=0$;

int $\mathrm{j}=0$;

float UVD;

float UVH;

float Gentec;

// globale Variablen aufrufen

extern int Chan;

extern float Spannung;

cout $<<$ "Anzahl der Eichpulse angeben" $<<$ endl;

$\operatorname{cin}>>\mathrm{n}$;

// Port B für Output konfigurieren 
int BoardNum = 0;

int ULStat $=0$;

int PortNum, Direction;

PortNum = FIRSTPORTB;

Direction = DIGITALOUT;

ULStat = cbDConfigPort (BoardNum, PortNum, Direction);

// Datenkopf in path schreiben

ofstream Outfile(path);

Outfile $<<$ Datenkopf $<<$ endl;

$$
\text { for }(j=0 ; j<n ; j++)\{
$$

// Pulsfolge starten

// Blende auf, Diode aktiv

ULStat = cbDOut(BoardNum, PortNum, 10);

Sleep (500);

// Diode Kanal 1 (UV) bei Dunkelheit einlesen

Chan $=1$;

Einlesen();

UVD=Spannung;

// Diode reset

ULStat = cbDOut(BoardNum, PortNum, 8);

Sleep (100);

// Diode aktiv, Laser scharf, d.h. Bit 0 auf 1 setzen, Laser triggert bei Übergang 1>0 ULStat = cbDOut (BoardNum, PortNum, 11);

Sleep (100);

// Laser feuern

ULStat = cbDOut $($ BoardNum, PortNum, 10);

Sleep (15);

// Gentec Kanal 0 und Diode (UV) Kanal 1 bei Helligkeit einlesen

Chan=0;

Einlesen();

Gentec=Spannung;

Chan $=1$;

Einlesen();

$\mathrm{UVH}=$ Spannung;

// Diode Reset, Blende bleibt auf

ULStat $=\operatorname{cbDOut}($ BoardNum, PortNum, 8);

Sleep (500);

// In durch path beschriebenen Ordner schreiben

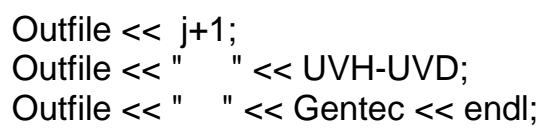

// Nummer des Eichpulses auf Bildschirm schreiben und dazu Meßwerte an Gentec und // UV- Detektor

printf("Pulsnummer");

printf("\%dln",j+1);

printf("Messwert am Gentec in mJ: "); 


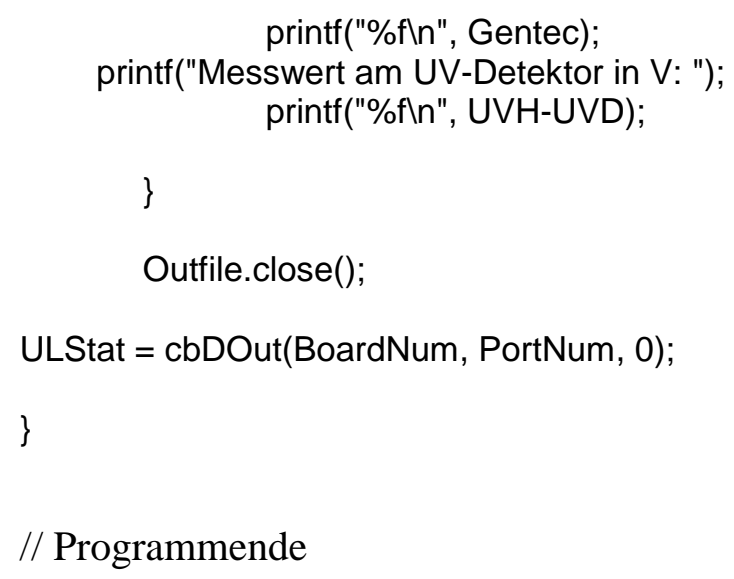

\section{Programm zur Aufnahme zeitaufgelöster Messsignale}

// Programm zur Aufnahme zeitaufgelöster Signale mit der 1621-4

// Transientenrekorderkarte und der 1602/12 ADC Karte, Stand:2002

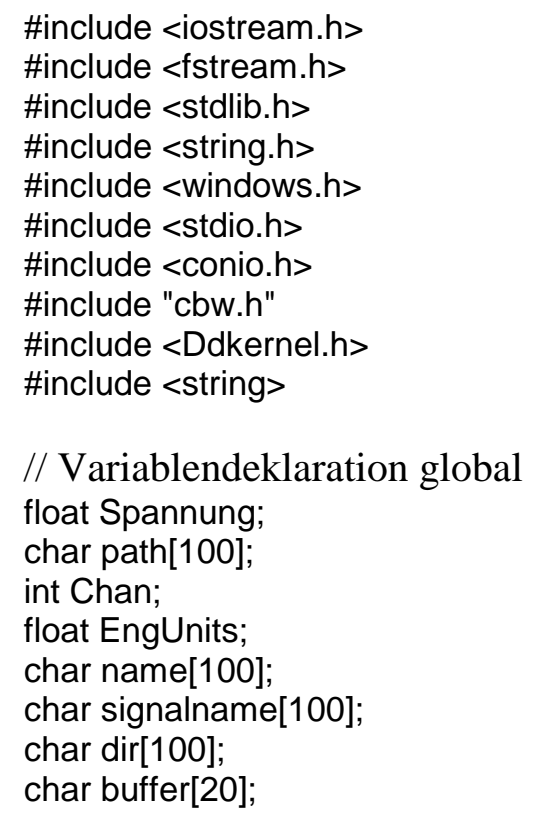

// Unterprogramm zur Bereitstellung eines Ordners für die Messung mit Eingabe des // Signalnamens

void Eingabe() \{

// Variablendeklaration lokal int $\mathrm{a}=0$;

//Namen für Ordner einlesen

cout $<<$ "Benutzername eingeben" $<<$ endl;

cin $>>$ name;

strcpy (dir, "||benutzerl|");

//strcpy (dir, "||benutzer|\Achim\"); //sonst "||benutzer|l"

strcat (dir, name);

strcat (dir, "I|Signale|l"); 


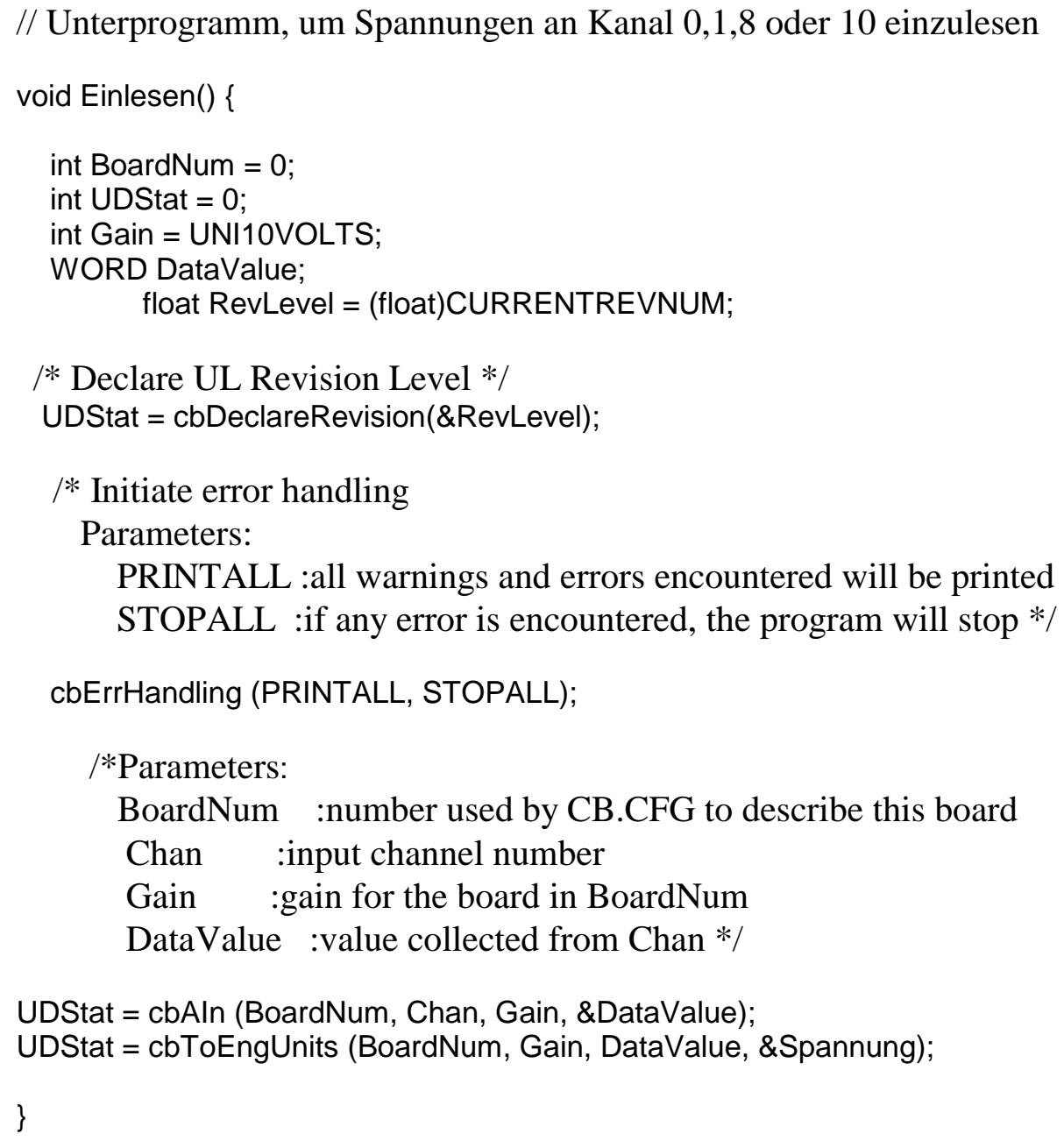

// Unterprogramm zur Ausgabe von Spannungen an die Kompensationsschaltung void Kompensation() \{

/* Variable Declarations */

int BoardNum = 0;

int ULStat $=0$;

int Gain = UNI10VOLTS;

int Chan $=0$;

WORD DataValue;

float RevLevel $=$ (float)CURRENTREVNUM;

/* Declare UL Revision Level */

ULStat $=$ cbDeclareRevision (\&RevLevel);

/* Initiate error handling

Parameters:

PRINTALL :all warnings and errors encountered will be printed

STOPALL :if any error is encountered, the program will stop */ // cbErrHandling (PRINTALL, STOPALL);

ULStat $=\mathrm{cbFromEngUnits} \mathrm{(BoardNum,} \mathrm{Gain,} \mathrm{EngUnits,} \mathrm{\& DataValue);}$

ULStat $=$ cbAOut (BoardNum, Chan, Gain, DataValue); 
void main() \{

Eingabe();

//1 okale Variablen

int $a=0$;

int $b=0$;

int $\mathrm{C}=0$;

int $d=0$;

int $e=0$;

int $\mathrm{f}=0$;

int $g=0$;

int $h=0$;

int $\mathrm{i}=0$;

int $\mathrm{j}=0$;

int $\mathrm{k}=0$;

int $\mathrm{n}$;

float la;

float lb;

float Ic;

float Id;

float le;

float If;

float lg;

float Ih;

float li;

float Ij;

float lk;

float II;

float Im;

float In;

float lo;

float Ip;

float lq;

float Ir;

float Is;

float It;

float lu;

float UVSpannung;

float UVH;

float UVD;

float 1008;

float 1008a;

float 1008z;

float 10010;

float 10010a;

float 10010z;

float 1010a;

float 1010z;

float 1010;

float 108a;

float 108z;

float 108;

float 108pruef;

float 108pruefa;

float 108pruefz;

float I1;
// Zählervariablen

// Spannung Kanal 8 vor Messung; Differenz I008a-I008z

// Spannung Kanal 8 vor Messung offene Blende

// Spannung Kanal 8 vor Messung geschlossene Blende

// Spannung Kanal 10 vor Messung

// Spannung Kanal 10 vor Messung offene Blende

// Spannung Kanal 10 vor Messung geschlossene Blende

// Spannung Kanal 10 vor Paket offene Blende

// Spannung Kanal 10 vor Paket geschlossne Blende

// Differenz; I010a-I010z;

// Spannung Kanal 8 vor Paket offene Blende

// Spannung Kanal 8 vor Paket geschlossene Blende

// Differenz; I08a-I08z;

// Spannung Kanal 8 Überprüfung

// Spannung Kanal 8 vor Überprüfung offene Blende

// Spannung Kanal 8 vor Überprüfung geschlossene Blende

// Spannung nach Einzelpuls Kanal 10 
float I2;

float I2a;

float I2z;
// Spannung nach Paket Kanal 8

// Spannung nach Paket offene Blende Kanal 8

// Spannung nach Paket geschlossene Blende Kanal 8

int Antwort;

float Bereich;

float UAnfang;

int reply;

int pruef;

int pruef2;

// globale Variablen aufrufen

extern float Spannung;

extern int Chan;

extern float EngUnits;

// Port B für Output konfigurieren

int BoardNum = 0;

int ULStat $=0$;

int PortNum, Direction;

PortNum = FIRSTPORTB;

Direction = DIGITALOUT;

ULStat = cbDConfigPort (BoardNum, PortNum, Direction);

//----------------------------------------MESSEN

Messen:

//IR-Blende auf

ULStat $=\operatorname{cbDOut}($ BoardNum, PortNum,4);

Sleep(1000);

// Ioo messen und eventuell korrigieren lassen

$\mathrm{lk}=0$;

$\mathrm{a}=0$;

for $(a=0 ; a<1000 ; a++)\{$

Chan=8;

Einlesen();

la=Spannung;

$\mathrm{lk}=\mathrm{lk}+\mathrm{la}$;

Sleep(1);

\}

$1008 \mathrm{a}=\mathrm{lk} / 1000$;

// IR-Blende zu

ULStat $=\operatorname{cbDOut}($ BoardNum, PortNum,0);

Sleep(2000);

II $=0$;

$\mathrm{b}=0$;

for $(b=0 ; b<1000 ; b++)\{$

Chan=8;

Einlesen();

$\mathrm{lb}=$ Spannung;

II=II+lb;

Sleep(1);

\}

$1008 \mathrm{z}=1 \mathrm{I} / 1000$

$1008=1008 \mathrm{a}-1008 \mathrm{z}$

Sleep(1000); 
// Blende wieder auf

ULStat $=\operatorname{cbDOut}($ BoardNum, PortNum,4);

cout $<<$ "I008 betraegt:";

cout $<<1008<<$ endl;

//-----------------------------------------FRAGEN

Fragen:

cout $<<$ "I008 O.K.? 1 fuer ja oder 2 fuer nein eingeben, keine Buchstaben!!! ";

cin $>>$ Antwort;

if (Antwort==1)

goto Weiter;

if (Antwort==2)

goto Messen;

goto Fragen;

$/ /$

WEITER

Weiter:

// Hier werden die Signalnamen hochgezaehlt test1, dann test2 usw., angelehnt an

// die Beispielprogramme unter den Suchbegriffen itoa und append. In diesem Bereich

// wird mit using namespace std der Variablentyp string definiert. Wenn std fuer das gesamte

// Programm genutzt wird, wird ofstream als mehrdeutige Variable angesehen.

\{ using namespace std;

// Signalnamen abfragen ohne Nummer die hochgezaehlt wird, z.B. test

cout $<<$ "Signalname eingeben" $<<$ endl;

cin $>>$ signalname;

//Hier wird die Nummer gestartet

int nummer;

nummer=0;

//--------------------------------------------NEU

neu:

// Signalname soll fuer weitere Verarbeitung string sein

string str(signalname); nummer=nummer+1;

// Nummer wird von integer in string umgewandelt und an buffer uebergeben

_itoa (nummer, buffer, 10);

str.append(buffer);

cout $<<$ str.c_str ()$<$ endl;

// Ordner gemäß der Eingabe zusammengesetzt aus Signalname und Nummer erstellen

$\bar{\xi}$

EngUnits=0;

Kompensation();

Sleep(500);

// Spannung an Kanal 10 vor der Messung bestimmen, um erste Kompensationsspannung // einzustellen offene Blende

ULStat = cbDOut $($ BoardNum, PortNum,4); 


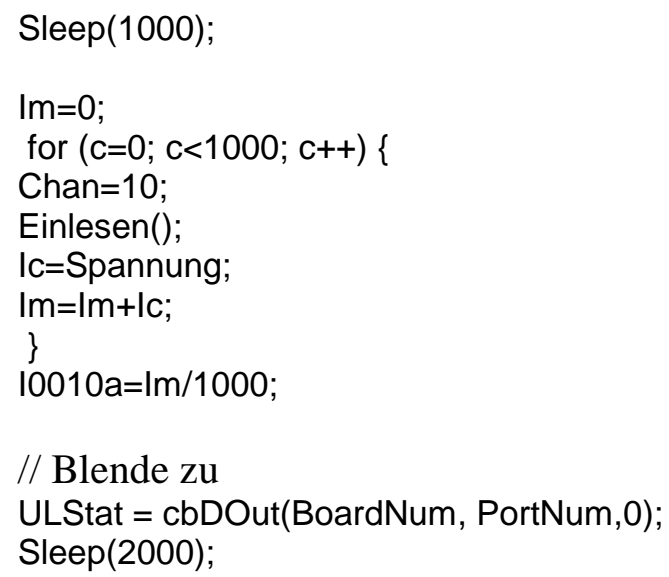

// geschlossene Blende

$\ln =0$;

for $(d=0 ; d<1000 ; d++)\{$

Chan=10;

Einlesen();

Id =Spannung;

In=In+ld;

\}

$10010 z=\ln / 1000$

$10010=10010 a-10010 z$

// Erste Kompensationsspannung rausgeben mit Bereich 1V rausgeben Sleep(500);

Bereich $=1$;

UAnfang $=1.01 *$ I0010-Bereich/4/10; //1.01 als Korrekturfaktor

EngUnits=UAnfang;

Kompensation();

Sleep(500);

ofstream Outfile(path);

Outfile $<<1008<<$ endl; $\quad$ // 1 .Zeile des Ausgabefiles

// Abfrage, ob I08 geprüft oder das Pulspaket gestartet werden soll cout $<<$ "Soll 108 geprueft werden? 1 fuer JA, 2 fuer NEIN (keine Buchstaben) " ;

cin $>>$ pruef;

if (pruef==1)

goto Pruefen;

if (pruef==2)

goto Pulszahl_einlesen;

$/ /$ PRUEFEN

Pruefen:

// Spannung an Kanal 8 wird abgefragt, und auf Bildschirm ausgegeben

// IR-Blende auf

ULStat $=\operatorname{cbDOut}($ BoardNum, PortNum,4);

Sleep (1000);

// Ioo messen und eventuell korrigieren lassen

$\mathrm{lk}=0$;

$\mathrm{a}=0$;

for $(a=0 ; a<1000 ; a++)\{$ 
Chan=8;

Einlesen();

la=Spannung;

$\mathrm{lk}=\mathrm{lk}+\mathrm{la}$;

Sleep(1);

\}

I08pruefa=lk/1000;

// IR-Blende zu

ULStat $=\operatorname{cbDOut}($ BoardNum, PortNum,0);

Sleep(2000);

II $=0$;

$\mathrm{b}=0$;

for $(b=0 ; b<1000 ; b++)\{$

Chan=8;

Einlesen();

$\mathrm{Ib}=$ Spannung;

II=II+lb;

Sleep(1);

\}

I08pruefz=II/1000;

108pruef=I08pruefa-I08pruefz;

Sleep(1000);

// Blende wieder auf

ULStat = cbDOut(BoardNum, PortNum,4);

cout $<<$ "I08 betraegt:";

cout $<<$ I08pruef $<<$ endl;

cout $<$ "Nochmal pruefen? 1 fuer JA, 2 fuer WEITER (keine Buchstaben) " ";

cin >> pruef2;

if (pruef2==1)

goto Pruefen;

if (pruef2 $==2$ )

goto Pulszahl_einlesen;

//-1-PULSZAHL_EINLESEN

Pulszahl_einlesen:

// Pulszahl einlesen

cout $<<$ "Anzahl der zu coaddierenden Pulse angeben" $<<$ endl;

$\operatorname{cin}>>\mathrm{n}$;

UVSpannung=0;

// Blende auf

ULStat $=\operatorname{cbDOut}($ BoardNum, PortNum,4);

Sleep(1000);

// Vor Pulspaket Spannung an Kanal 10 bei offener Blende einlesen, I010a $\mathrm{lo}=0$;

for $(\mathrm{e}=0 ; \mathrm{e}<1000 ; \mathrm{e}++)\{$

Chan=10;

Einlesen();

le=Spannung; 
lo=lo+le;

\}

$1010 \mathrm{a}=\mathrm{lo} / 1000$;

// Vor Pulspaket Spannung an Kanal 8 bei offener Blende einlesen, I08a

$\mathrm{Ip}=0$;

for $\left(f=0 ; f<1000 ; f_{++}\right)\{$

Chan=8;

Einlesen();

If=Spannung;

Ip=Ip+lf;

Sleep (1);

\}

$108 \mathrm{a}=\mathrm{lp} / 1000$

//Blende zu

ULStat $=\operatorname{cbDOut}($ BoardNum, PortNum,0);

Sleep(2000);

// Vor Pulspaket Spannung an Kanal 10 bei geschlossener Blende einlesen, I010z $\mathrm{lq}=0$;

for $(g=0 ; g<1000 ; g++)\{$

Chan=10;

Einlesen();

$\lg =$ Spannung;

$\mathrm{lq}=\mathrm{lq}+\mathrm{lg}$;

\}

$1010 z=1 q / 1000$

// Vor Pulspaket Spannung an Kanal 8 bei geschlossener Blende einlesen, I08z

$\mathrm{Ir}=0$;

for $(h=0 ; h<1000 ; h++)\{$

Chan=8;

Einlesen();

$\mathrm{lh}=$ Spannung;

$\mathrm{Ir}=\mathrm{Ir}+\mathrm{lh}$;

Sleep(1);

\}

$108 z=\operatorname{lr} / 1000$;

// I08 und I010 als Differenzen zwischen offener und geschlossener Blende abspeichern $108=108 a-108 z$

Outfile $<<108<<$ endl;

// 2.Zeile des Ausgabefiles

I010=1010a-1010z;

cout $<<1010<<$ endl;

Outfile $<<1010<<$ endl;

// 3.Zeile des Ausgabefiles

// Pulsfolge starten

$$
\text { for }(j=0 ; j<n ; j++)\{
$$

//IR-Blende auf, Diode aktiv

// I0 100 mal messen und Mittelwert bilden, Ik ist die Einzelmessung, Is die Summe und I0

// der Mittelwert

cout $<<$ "Pulsnummer: ";

cout $<<$ j+1 $<<$ endl; 
// Kompensieren

Sleep(500);

// IR-Blende auf, Diode aktiv

ULStat = cbDOut(BoardNum, PortNum,6);

// I1 100 mal messen und Mittelwert bilden, Il ist die Einzelmessung, It die Summe und I1 der // Mittelwert

Sleep (500);

// Diode Kanal 1 (UV) bei Dunkelheit einlesen

Chan $=1$;

Einlesen();

UVD=Spannung;

// Diode reset, Blenden auf Laser scharf, d.h. Bit 0 auf 1 setzen, Laser triggert bei Übergang

$/ / 1>0$

ULStat $=\operatorname{cbDOut}($ BoardNum, PortNum, 12);

Sleep (500);

// Laser feuern Hier findet Signalaufnahme statt,

// Aufnahmeprogramm Sbench wird getriggert

ULStat = cbDOut(BoardNum, PortNum, 15);

Sleep (1000);

ULStat $=$ cbDOut $($ BoardNum, PortNum, 14);

Sleep(6000);

// Diode (UV) Kanal 1 bei Helligkeit einlesen

Chan $=1$;

Einlesen();

$\mathrm{UVH}=$ Spannung;

// Zur Berechnung der Laserenergie die Differenz zwischen hell und dunkel an der // UV-Diode aufsummieren

UVSpannung=UVSpannung+UVH-UVD;

// Diode reset, UV-Blende zu;

ULStat $=\operatorname{cbDOut}($ BoardNum, PortNum, 12);

ULStat $=\operatorname{cbDOut}($ BoardNum, PortNum, 4);

EngUnits $=0$;

Kompensation();

Sleep (1000);

Is $=0$;

for $(\mathrm{i}=0 ; \mathrm{i}<1000 ; \mathrm{i}++)\{$

Chan $=10$;

Einlesen();

$\mathrm{li}=$ Spannung;

//cout $<<$ II;

//cout $<<$ It;

Is=Is+li;

\}

$11=\mathrm{Is} / 1000$;

cout $<<$ I1 $<$ endl;

Sleep (1000);

// Kompensation auf Bereich 1V vor naechstem Puls

EngUnits $=1.01 * 11$-Bereich/4/10;

Sleep(500); 
Kompensation();

Sleep(3000);

//Nach Pulsfolge zur Bestimmung des mittleren Umsatzes erneut Spannung an // Kanal 8 messen bei offener und geschlossener Blende $\mathrm{It}=0$;

for $(j=0 ; j<1000 ; j++)\{$

Chan=8;

Einlesen();

lj=Spannung;

//cout $<<$ II;

//cout $<<$ It;

$\mathrm{lt}=\mathrm{It}+\mathrm{lj}$

Sleep(1);

\}

$12 \mathrm{a}=\mathrm{It} / 1000$;

//Blende zu

ULStat $=\operatorname{cbDOut}($ BoardNum, PortNum, 0$)$;

Sleep (2000);

lu $=0$;

for $(k=0 ; k<1000 ; k++)\{$

Chan=8;

Einlesen();

Ik=Spannung;

lu=lu+lk;

Sleep(1);

\}

$12 z=l u / 1000$

$12=12 a-12 z ;$

cout $<<$ I $2<$ endl;

// In durch path beschriebenes Ausgabefile schreiben

Outfile $<<$ UVSpannung/n $<<$ endl; $\quad / / 4$. Zeile

Outfile $<<$ I $2<<$ endl;

// 5. Zeile

Outfile $<<10010<<$ endl;

// 6. Zeile

Outfile $<<108 \mathrm{a}<<$ endl;

// 7. Zeile

Outfile $<<12 \mathrm{a}<<$ endl;

// 8. Zeile

Outfile $<<1008 \mathrm{a}<<$ endl;

// 9. Zeile

Outfile $<<\mathrm{n}<<$ endl;

// 10. Zeile

// Blende auf

ULStat $=\operatorname{cbDOut}($ BoardNum, PortNum,4);

cout $<<$ "Weiter messen? 1 fuer ja oder 2 fuer nein eingeben, keine Buchstaben!";

cin >> reply;

if (reply==1) goto neu;

ULStat $=\operatorname{cbDOut}($ BoardNum, PortNum,0);

Outfile.close();

EngUnits $=0$;

Kompensation();

\}

// Programmende 


\section{Matlab $^{\circledR}$-Programmcode}

\section{Programm zur Auswertung von SP-PLP-Signalen}

$\%$ Programm zur Auswertung von SP-PLP Signalen

$\%$ in Verbindung mit absoluten Spannungen von der ADC-Karte

$\%$ Nur lauffähig mit dem Unterprogramm " fitsignal"

$\%$

$\%$ Dieses Hauptprogramm basiert in wesentlichen Teilen auf

$\%$ einer von Achim Feldermann geschrieben Auswerteroutine.

$\%$ Es wurde von Thomas Junkers für Matlab ${ }^{\circledR}$ 6.1 Release 12 (Firma MathWorks Inc.)

$\%$ umgeschrieben und die Prozedur von zehn Anpassungen pro Signal hinzugefügt.

$\%$

clear;

pot $=0$

$\%$ Startparameter der Fitprozeduren spezifizieren

$\mathrm{q}=1$;

$\mathrm{W}=0.01$

$\%$ Datei mit Messdaten von TR-Recorder importieren

[file1_txt, path1] = uigetfile('*.txt',' File ('.txt) mit Messdaten TR');

eval(['load ', path1, file1_txt]);

format long;

$\%$ Dateiextension (.txt) entfernen

for $\quad \mathrm{i}=1$ :length(file1_txt)-4

end;

file1(i) = file1_txt(i);

$\%$ Datei mit Messdaten von ADC-Karte importieren, Name muss mit Buchstaben beginnen [file2_txt, path2] = uigetfile('*.txt',' File (".txt) mit Messdaten ADC');

eval(['load ', path2, file2_txt]);

$\%$ Dateiextension (.txt) entfernen

for $\quad \mathrm{i}=1$ :length(file2 txt) -4

end;

file2(i) = file2_txt(i);

$\%$ Inhalt der Dateien an dummy1 und dummy2 übergeben

dummy2 = eval(file2);

dummy1 = eval(file1);

$\%$ Anzahl der Messwerte in Messdatei einlesen

$\mathrm{m}=$ length (dummy1)

$\mathrm{n}=$ length(dummy2);

It = dummy $1(:) ; \quad \%$ zeitaufgelöste Spannung von TR

labs = dummy2(:::); \% absolute Spannung von ADC Kanal 8

$\%$

$\%$ Experimentelle Parameter eingeben

$\%$ 


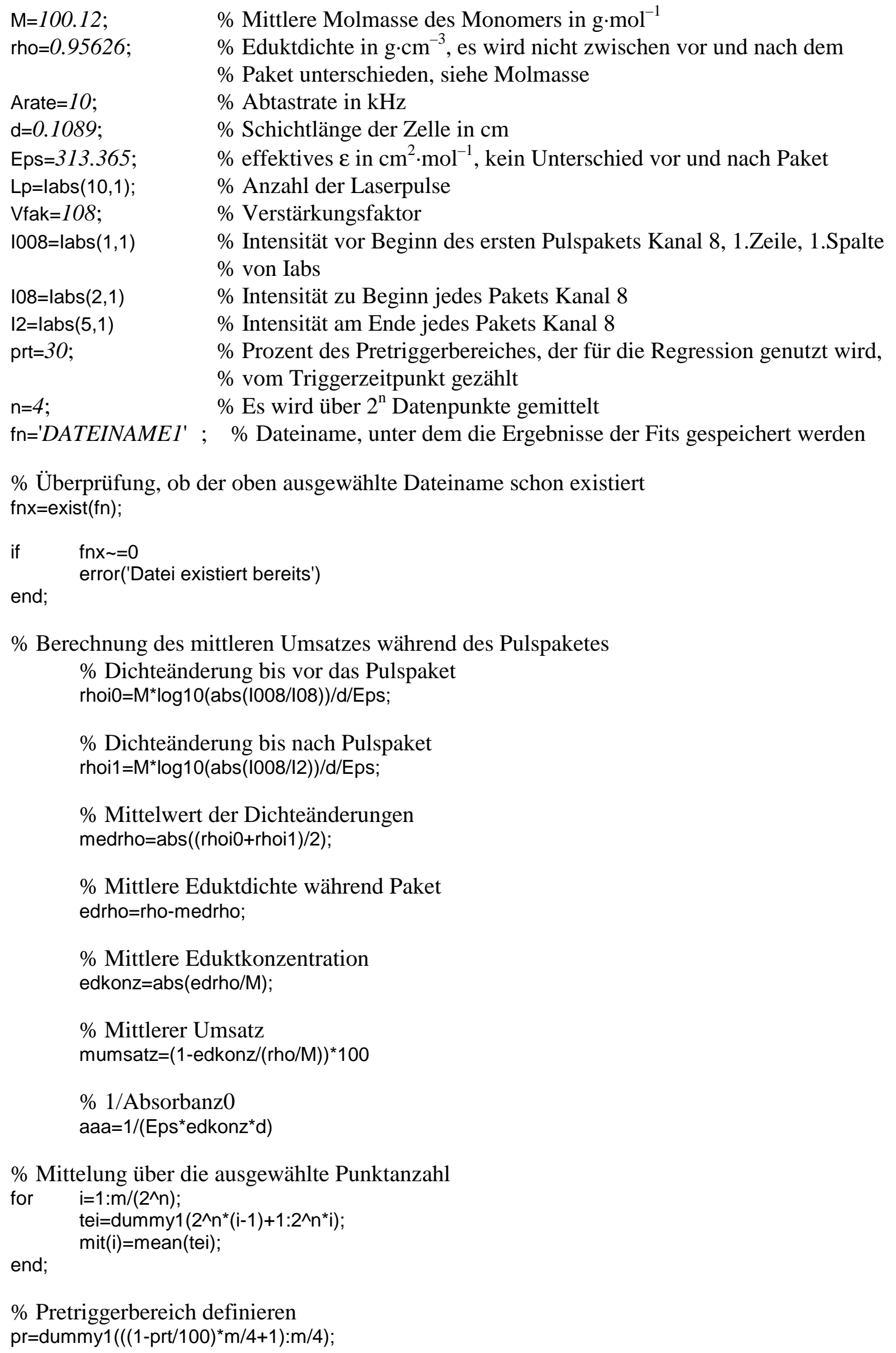


mi=mean(pr) \% Mittelwert des ausgewählten Pretriggerbereichs

$\%$ Variablennamen für $\mathrm{t}$ und y in Abhängigkeit von Auswerteprozent finden vartxxx='vart000';

varyxxx='vary000';

for $\quad h a r=1: 10$

pot=pot+10;

$\mathrm{X}=\mathrm{pot}$;

for $\quad$ zaehl=1:length(vartxxx)-1

zaehler(zaehl)=vartxxx(zaehl);

if $\quad z a e h l==$ length $(\operatorname{vartxxx})-1$

counter=zaehler(zaehl);

counter=counter +1 ;

end;

$\operatorname{vartxxx}($ zaehl $)=$ counter;

end;

for $\quad z a e h l=1$ :length (varyxxx)

zaehler(zaehl)=varyxxx(zaehl);

if $\quad z a e h l==$ length (varyxxx)-1

counter=zaehler(zaehl);

counter=counter +1 ;

end;

varyxxx (zaehl)=counter;

end;

if $\quad$ pot $==100$

vartxxx='vart100';

end;

varyxxx='vary100';

$\%$ Punkte auswählen, für die Fit durchgeführt wird, x Prozent des Postriggerbereichs+ Punkte

$\%$ bis Triggerzeitpunkt

for $\quad i=\left(m /\left(4^{\star} 2^{\wedge} n\right)+1\right):\left(\left(m /\left(2^{\wedge} n\right)-m /\left(4^{*} 2^{\wedge} n\right)\right)^{\star} x / 100+m /\left(4^{\star} 2^{\wedge} n\right)\right)$;

$\%$ Zeit für jeweils einen Messpunkt bestimmen

$t(i)=1 /\left(1000^{*} \text { Arate }\right)^{*}\left((i-1)^{*} 2^{\wedge} n+2^{\wedge}(n-1)-0.5\right)-1 /\left(1000^{*} \text { Arate }\right)^{\star}(m /(4)) ;$

$\%$ Umrechnung jeweils eines Spannungspunktes in das entsprechende $\mathrm{c}_{\mathrm{M}} / \mathrm{c}_{\mathrm{M}}{ }^{0}$

end; $y(i)=1-a a a / L p^{*} \log 10\left(\operatorname{abs}\left(1+L p^{*}(\right.\right.$ mit(i) - mi $) / I 08 /$ Vfak $\left.)\right)$;

for $\quad i=1:\left(m /\left(4^{*} 2^{\wedge} n\right)\right)$;

$t(i)=1 /\left(1000^{*} \text { Arate }\right)^{*}\left((i-1)^{*} 2^{\wedge} n+2^{\wedge}(n-1)-0.5\right)-1 /\left(1000^{*} \text { Arate }\right)^{*}(m /(4))$;

end;

$y(i)=1+\left(a^{*} \log 10\left(\operatorname{abs}\left(1+L^{*}(\operatorname{mit}(\mathrm{i})-\mathrm{mi}) /\left(108^{*} \mathrm{Vfak}\right)\right)\right) /(\mathrm{Lp})\right)$;

$\mathrm{t}=\mathrm{t}(:)$;

$\mathrm{y}=\mathrm{y}(:)$;

assignin('base',vartxxx,t);

assignin('base',varyxxx,y);

clear $\mathrm{t}$;

end;

clear y; 
\% Abspeichern der Rohdaten für Auftragungen in anderen Programmen $\mathrm{s} 1=\mathrm{vart} 100$

$\mathrm{s} 2=$ vary $100 ;$

file=[s1 s2];

$\%$ Namensgebung der Datei hier:

save DATEINAME2 file -ascii -double -tabs

$\%$ Voransicht des umgerechneten Signals

$\mathrm{f}_{-}=\mathrm{clf}$;

figure(f_);

legh_= $=[$; legt $=\{\} ;$

$x \lim _{-}=[\operatorname{lnf}-\operatorname{Inf}]$;

ax_ = subplot $(1,1,1)$;

set(ax_,'Box','on');

axes(ax_); hold on;

$\%$ Plot der Daten " vary100 vs. vart100" , also des gesamten Signals

h_ = line (vart100,vary100,'Parent',ax_,'Color',[0.666667 0 0.333333],...

'LineStyle','none', 'LineWidth', $1, \ldots$

'Marker',.'.' 'MarkerSize',12);

$x \lim \_(1)=\min \left(x \lim \_(1), \min (\operatorname{vart100})\right)$;

$x \lim \_(2)=\max \left(x \lim \_(2), \max (\operatorname{vart100})\right)$;

legh_(end +1$)=h \_$;

legt_ $\{$ end +1$\}=$ 'vary100 vs. vart100';

$\%$ Start der Anpassungen

flop=input('Soll dieses Signal wirklich gefittet werden? (0 für NEIN) ');

if $\quad$ flop $==0$

fid=fopen (fn, ' $a$ ');

fprintf(fid, 'Fit nicht durchgeführtır');

fclose(fid);

end;

error('Keine Anpassung vorgenommen');

fitsignal(vart010,vary010,vart020,vary020,vart040,vary040,vart030,vary030,vart050,vary050, .. vart060, vary060, vart070, vary070, vart080, vary080, vart090, vary090, vart100,vary $100, \ldots$ mumsatz,fn, $\mathrm{q}, \mathrm{w})$;

$\%$ Programmende 


\section{Auszug aus dem Unterprogramm ,fitsignal“6}

Diese Unterprogramm wurde von Thomas Junkers (Diplomarbeit) geschrieben. Das komplette Programm ist dort abgedruckt. Hier ist nur die Struktur der eigentlichen Fitprozedur gezeigt. $\%$

$\%$ Fit Prozeduren

$\%$

$\%$ allgemeine Definitionen

fo_ = fitoptions('method','NonlinearLeastSquares','Robust','On','Algorithm','Levenberg-Marquardt',...

st_e $[\mathrm{q} w]$ 'MaxFunEvals',10000,'Maxlter',1000,'ToIFun',1e-100,'TolX',1e-100);

set(fo_,'Startpoint',st_);

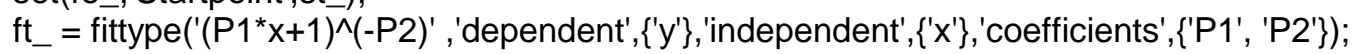

$\%$ Create fit $10 \%$

disp('Computing Fit 1 of $\left.10^{\prime}\right)$;

ex_ $=0$ > vart010;

set(fo_,'Exclude',ex_);

$\%$ Ausführen des Fits

[cf_gof,output] = fit(vart010,vary010,ft_,fo_);

disp(cf_); 


\section{DANKSAGUNG}

Ich danke Herrn Prof. Dr. M. Buback für die interessante Themenstellung sowie die stete Diskussionsbereitschaft und Förderung dieser Arbeit.

Für die Unterstützung bei der Bewältigung von Softwareproblemen möchte ich mich bei Herrn Dr. H.-P. Vögele und Matthias Müller bedanken.

Allen Mitgliedern der Arbeitsgruppe Buback danke ich für die nette Arbeitsatmosphäre und die stete Hilfsbereitschaft. Ein besonderer Dank gilt dabei Heike Rohmann für die Reinigung der Monomere und die Hilfe bei den Schwierigkeiten mit der Trockenbox. Ich danke Dr. Mark Egorov für die Unterstützung bei der Auswertung der Messsignale und die vielen anregenden Diskussionen über $k_{\mathrm{t}}$.

Für die gute Zusammenarbeit und den Spaß bei der Arbeit möchte ich mich besonders bei Thomas Junkers bedanken.

Meinen ehemaligen Bürokollegen Dr. Christoph Isemer und Dr. Christopher Barner-Kowollik gilt mein spezieller Dank für ihre Freundschaft und ihren Sinn für Humor.

Den Mitarbeitern der Institutswerkstätten möchte ich für ihre Hilfsbereitschaft und die stets schnelle und zuverlässige Arbeit meinen Dank aussprechen.

Der deutschen Forschungsgemeinschaft danke ich für die finanzielle Unterstützung im Rahmen des Europäischen Graduiertenkollegs „Microstructural Control in Free-Radical Polymerization“.

Besonders herzlich bedanke ich mich bei meinen Eltern, die mir dieses Studium ermöglicht und mich jederzeit unterstützt haben.

Rahel danke ich dafür, dass sie immer für mich da war und für alles, was wir gemeinsam erleben und noch erleben werden. 


\section{LEBENSLAUF}

Persönliche Daten:

11.11.1973

Schulausbildung:

1980-1984

1984-1986

1986-1993

Mai 1993

\section{Zivildienst:}

Jul. 1993 - Sept. 1994

Zivildienst beim Rettungsdienst des Landkreises Diepholz geboren in Bremen

Grundschule in Weyhe bei Bremen

Orientierungsstufe in Weyhe

Gymnasium in Weyhe

Erwerb der Allgemeinen Hochschulreife

\section{Hochschulausbildung:}

Okt. 1994

Feb. 1997

Aug. 1998 - Jul. 1999

Okt. 1998 - Jul. 1999

Jul. 1999

seit Jul. 1999

seit Jul. 1999

Jan. 2000-Dez. 2002

Okt. 1997-Feb. 2001

Feb. 2001

Beginn des Chemiestudiums an der Universität Göttingen

Diplomvorprüfung Chemie

Anfertigung der Diplomarbeit am Institut für Physikalische Chemie der Universität Göttingen zum Thema „Untersuchung der SubstanzCopolymerizationen der Systeme Methylmethacrylat-Dodecylacrylat und Dodecylmethacrylat-Methylacrylat durch Pulslaser-Techniken“ unter Anleitung von Prof. Dr. M. Buback

Tätigkeit als studentische Hilfskraft am Institut für Physikalische Chemie der Universität Göttingen

Diplomhauptprüfung Chemie

Anfertigung der Dissertation am Institut für Physikalische Chemie der Universität Göttingen zum Thema „Terminierungskinetik radikalischer Homo- und Copolymerisationen bis $\mathrm{zu}$ hohen Monomerumsätzen“" unter Anleitung von Prof. Dr. M. Buback

wissenschaftliche Hilfskraft am Institut für Physikalische Chemie der Universität Göttingen

Stipendiat im Europäischen Graduiertenkolleg „Microstructural Control in Free-Radical Polymerization“"

Studium der Betriebswirtschaftslehre an der Universität Göttingen

Diplomvorprüfung Betriebswirtschaftslehre 